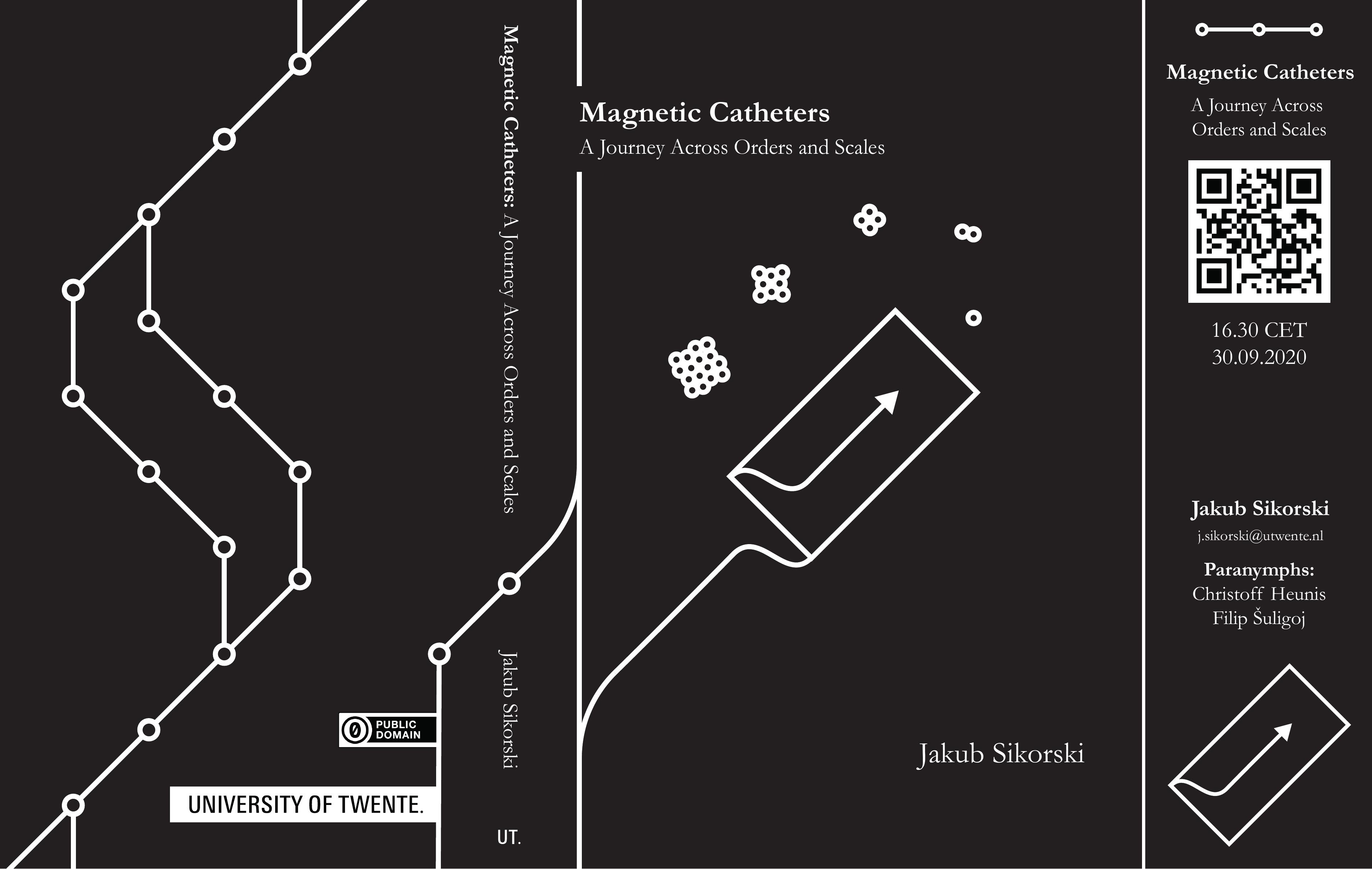


MAGNETIC CATHETERS

A JOURNEY ACROSS ORDERS AND SCALES 



\title{
MAGNETIC CATHETERS \\ A Journey Across Orders and Scales
}

\section{Dissertation}

to obtain

the degree of doctor at the University of Twente, on the authority of the rector magnificus,

Prof. dr. T.T.M. Palstra,

on account of the decision of the Doctorate Board

to be publicly defended

on Wednesday 30th of September 2020 at 16.45

\author{
by
}

\section{Jakub Sikorski}

born on the 25th of October 1992

in Duszniki-Zdrój, Poland 


\section{This dissertation has been approved by: \\ Prof. Dr. Sarthak Misra}

Cover design: Jakub Sikorski

Printed by: Ipskamp Printing, Enschede

ISBN: 978-94-6421-037-8

DOI: $10.3990 / 1.9789464210378$

\section{(0) PusLcoomal 2020 Jakub Sikorski, The Netherlands.}

The author has dedicated all copyright and related and neighboring rights to this work to the public domain by waiving all of his rights to the work worldwide under copyright law, including all related and neighboring rights, to the extent allowed by law.

You can copy, modify and distribute the work, even for commercial purposes, all without asking permission. 


\section{Graduation Committee}

Chairman / secretary:

Prof. Dr. Ir. H.F.J.M. Koopman

(University of Twente)

\section{Supervisor:}

Prof. Dr. S. Misra

(University of Twente and University Medical Centre Groningen)

\section{Committee Members:}

Prof. Dr. P.E. Dupont

(Boston Children's Hospital, Harvard Medical School)

Prof. Dr. Ir. M. Steinbuch

(Eindhoven University of Technology)

Prof. Dr. J.G. Grandjean

(University of Twente)

Prof. Dr. Ing. J. Arens

(University of Twente)

Dr. V. Kalpathy Venkiteswaran

(University of Twente) 


\section{This work is part of the research project ROBOTAR}

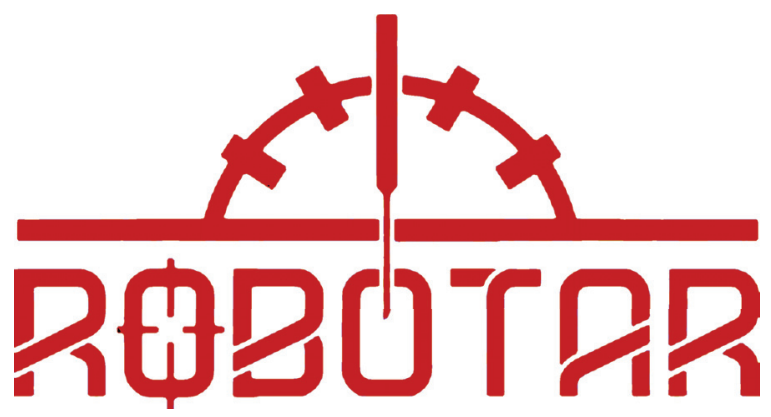

The reported research has received funding from the European Research Council

(ERC) under the European Union's Horizon 2020 Research and Innovation programme (Grant Agreement \#638428 -- project ROBOTAR: Robot-Assisted

Flexible Needle Steering for Targeted Delivery of Magnetic Agents).
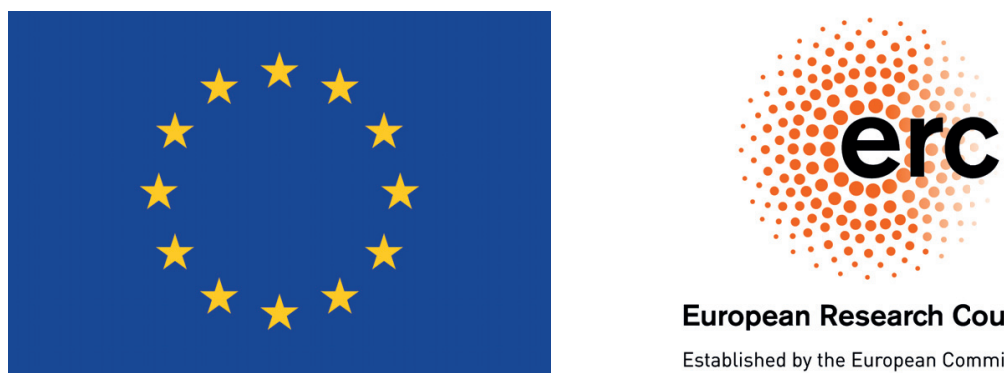

\section{European Research Council}

Established by the European Commission 


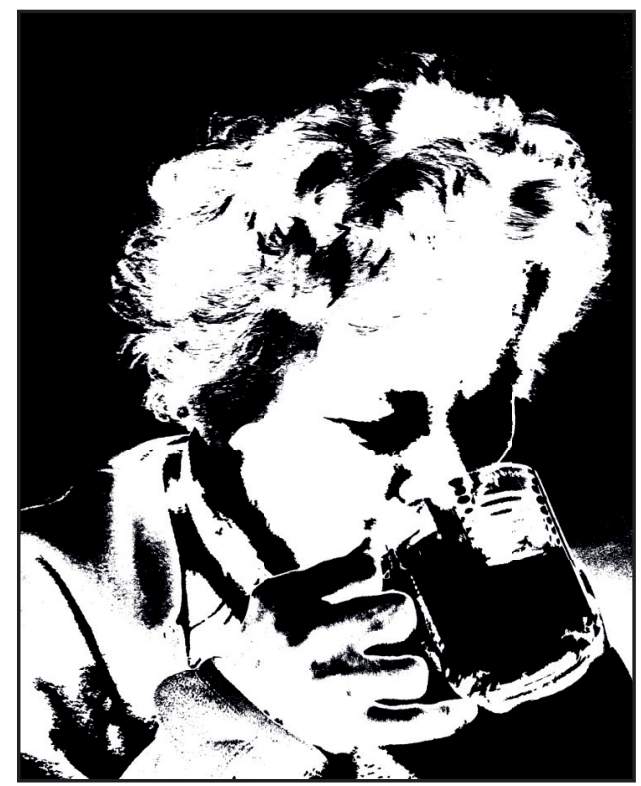

In memory of

my grandmother Anna

and my friend Guilherme

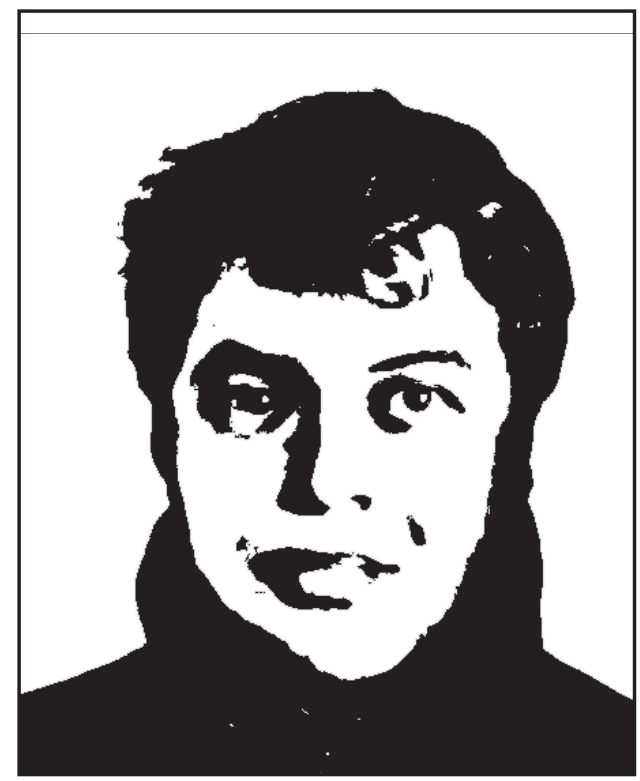





\section{Summary}

Research in minimally-invasive surgery (MIS) develops means to diagnose and treat patients quickly, precisely and through minimal access ports, with the ultimate aim of making surgical procedures cheaper, less distressful and more effective than current state-of-the-art. Technological progress in engineering and life sciences empowers the MIS tool-kit, which already enables several revolutionary medical procedures, such as endoscopy, cardiac ablation, image-guided biopsies or angioplasty. When an MIS procedure requires access to a deep-seated region, various groups of flexible surgical instruments are employed to operate inside the body through narrow incisions. Related challenges can be approached with robotic solutions, whereby real-time sensing and automation improve the in situ behaviour of flexible instruments.

This thesis studies a subgroup of flexible instruments called catheters. Catheters are sleek tubes navigated through the natural cavities of human body to reach remote organs with minimum tissue damage. However, due to their high mechanical compliance, catheters are challenging to steer once inside the body. This limits their applicability to a narrow range of tasks, predominantly in endovascular surgery. Dexterity of a catheter can be increased by integrating into its structure coils or permanent magnets, which experience forces/torques in external magnetic field. Such magnetic catheters are capable of exhibiting complex mechanical behaviour, while being structurally simpler than comparable devices. The following chapters demonstrate how, by using the principles of robotics, this behaviour can be harnessed to execute clinically-relevant tasks. Contributions are made in three general areas. The thesis proposes novel designs of magnetic catheters, explores the means to sense and model their behaviour and devises control strategies enabling their operation.

Magnetic catheters bridge scales. They belong to mesoscale, as their functional part is situated at their tip, which has a diameter expressed in millimetres and the length of several centimetres. However, their total length can exceed a meter, and thus, from that perspective, they can be also considered macroscale. This multiscale geometry allows magnetic catheters to successfully operate inside the human body, while being actuated externally by macroscale auxiliary systems. Yet, the catheters ultimately target living tissue, which exhibits complex behaviour mediated at micro- and nano- scales. Thus, to explore the broad domain of magnetic catheters searching for clinically-relevant solutions, this thesis takes the reader on a journey through orders of magnitude. After a general introduction (Chapter 1), we present a series of studies, divided into three parts, with each part focusing on challenges relevant to a particular scale. 
Part I describes the development of two generations of auxillary systems for magnetic actuation. These macroscale systems employ electromagnets located outside of the body of the patient to transmit power to a range of magnetic catheters. BigMag (Chapter 2) is an array of mobile electromagnets utilising optimization-based inverse field map to generate prescribed magnetic fields on demand. Knowledge gained during the development of BigMag is then used to inform design of the ARMM (Advanced Robotics for Magnetic Manipulation) system (Chapter 3). The ARMM system employs a single mobile electromagnet located on a robotic arm, allowing for magnetic steering in a workspace suitable for minimally-invasive surgical procedures. Both of these systems are applied in Part II, which explores mesoscale, focusing on design, modelling and control of millimetre-sized distal parts (tips) of magnetic catheters for clinically-relevant tasks of motion control (Chapter 4), grasping (Chapter 5) and ultrasound-guided projectile delivery (Chapter 6). Subsequently, Part III reconsiders the role of magnetic catheter within MIS, treating it as auxiliary infrastructure to actuate even smaller objects, thus providing means for controlled interaction with human body in microscale. MILiMAC (Microrobotic Infrastructure Loaded into Magnetically-Actuated Catheter) is a mesoscale device that can be considered both an electromagnet array and a magnetic catheter (Chapter 7). It is a proofof-concept solution addressing the challenge of steering microrobotic agents in remote regions of human body. Ultimately, the range of topics approached by this thesis is summarised in Part IV, providing general conclusions and outlook (Chapter 8). 


\section{Contents}

$\begin{array}{ll}\text { Summary } & 9\end{array}$

1 Introduction $\mathbf{1 5}$

1.1 Robotic Technology in Minimally-Invasive Surgical Procedures . . 16

1.2 Catheters as Continuum Robots . . . . . . . . . . . . . . . 19

1.3 Magnetic Actuation . . . . . . . . . . . . . . . . . 21

1.4 Research Objectives and Outline of the Thesis . . . . . . . . 23

1.5 Scientific Output . . . . . . . . . . . . . . 27

I Starting Big: Magnetic Actuation Systems 29

2 BigMag: A Novel System for 3D Magnetic Actuation of Flexible Surgical Manipulators $\quad 33$

2.1 Introduction . . . . . . . . . . . . . . . . . . . . . . . . . . . . 34

2.2 Design of BigMag . . . . . . . . . . . . . . . . . 35

2.2.1 Design Rationale . . . . . . . . . . . . . . . 36

2.2.2 BigMag - General Overview . . . . . . . . . . . . . . 37

2.3 Modelling of BigMag Coil Array . . . . . . . . . . . . . . . . 38

2.3.1 Forward Map of BigMag Coil Array . . . . . . . . . . . . 38

2.3.2 Inverse Map of BigMag Coil Array . . . . . . . . . . . . . . 40

2.3.3 Field Map Validation . . . . . . . . . . . . . . . . 41

2.3.4 Steering of Magnetic Catheters - Preliminary Test . . . . . 42

2.4 Conclusions and Future Work . . . . . . . . . . . . . . 43

3 The ARMM System: An Optimally-Designed Mobile Electromagnetic Coil for Non-linear Actuation of Flexible Surgical In$\begin{array}{ll}\text { struments } & \mathbf{4 5}\end{array}$

3.1 Introduction . . . . . . . . . . . . . . . . . . 46

3.2 Design of the ARMM system . . . . . . . . . . . . . . . . . 48

3.2.1 System Composition and Magnetic Steering Strategy . . . . 48

3.2.2 Payload-Optimized Design of a Mobile Electromagnet . . . 50

3.2.3 Final Coil Design . . . . . . . . . . . . . . . . 52

3.3 Non-linear Magnetic Actuation

Using The ARMM System . . . . . . . . . . . . . . . . 53

3.4 Implementation and Experimental Results . . . . . . . . . . . . 56

3.4.1 Performance and Limitations of ARMM . . . . . . . . . 57 
3.4.2 Experimental Validation . . . . . . . . . . . . 58

3.5 Conclusions and Future Work . . . . . . . . . . . . . . . . 59

\section{Small but Sassy: Mesoscale Magnetic Catheters 63}

4 Vision-Based 3D Steering of Magnetic Catheter Using BigMag 67

4.1 Introduction . . . . . . . . . . . . . . . . . . . 68

4.2 Modelling a magnetic catheter . . . . . . . . . . . . . . 69

4.3 Vision-based catheter shape sensing . . . . . . . . . . . . 73

4.4 Closed-Loop Steering of Magnetic Catheters . . . . . . . . . . . . . 76

4.4.1 Inverse Model Control . . . . . . . . . . . . . . . 77

4.5 Experimental Validation . . . . . . . . . . . . . . . . . 79

4.5.1 Shape Sensing Validation . . . . . . . . . . . . 79

4.5.2 Closed-Loop Steering . . . . . . . . . . . . . 80

4.6 Conclusions ....................... 83

5 Grasping Using Magnetically-Actuated Tentacle Catheter: A Proof-Of-Concept Study $\quad \mathbf{8 5}$

5.1 Introduction . . . . . . . . . . . . . . . . . 86

5.2 Catheter as a Grasping Tool - Conceptual Design . . . . . . . . . . 88

5.2.1 Design Requirements and Preliminary Concepts . . . . . 88

5.2.2 Details of Tentacle Catheter . . . . . . . . . . . . . 90

5.3 Tentacle Catheter - Experimental Proof-of-Concept . . . . . . . . . 92

5.3.1 Thermal Safety Study . . . . . . . . . . . . . . 92

5.3.2 Estimation of Maximum Grasping Force . . . . . . . . . . . 93

5.3.3 Pick-and-Place Task . . . . . . . . . . . . . . . . 97

5.4 Conclusions and Future Work . . . . . . . . . . . . . . 97

6 Flexible Catheter for Ultrasound-Guided Delivery of Magnetic $\begin{array}{lr}\text { Projectiles } & 99\end{array}$

6.1 Introduction . . . . . . . . . . . . . . . . . . . 100

6.2 Targeted Drug Delivery Using Magnetic Catheter . . . . . . . . . . 102

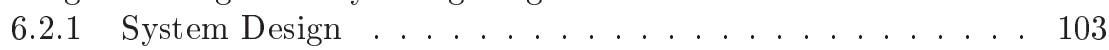

6.2.2 Prototype Fabrication . . . . . . . . . . . . . . . 104

6.3 Catheter Tracking . . . . . . . . . . . . . . . . . . . 104

6.4 Magnetic Catheter Steering Using the ARMM System . . . . . . . 107

6.4.1 Stage 1: Catheter Navigation . . . . . . . . . . . . . 108

6.4.2 Stage II: Projectile Targeting . . . . . . . . . . . . . . . . 113

6.5 Experimental Setup . . . . . . . . . . . . . . . 115

6.6 Results................................ 115

6.6.1 Validation of Catheter Shape Reconstruction

Using Scanning Ultrasound . . . . . . . . . . . . . . 115

6.6.2 Closed-loop Position Control of Magnetic Catheter . . . . . 118

6.6.3 Projectile Targetting: Computational Analysis . . . . . . . 118

6.6.4 Projectile Delivery: Experiments . . . . . . . . . . . 119

6.7 Discussion . . . . . . . . . . . . . . . . . . 120

6.8 Conclusions . . . . . . . . . . . . . . . . . . . 123 


\section{A Matter of Perspective: Catheters as Magnetic Ac-} tuation Systems

7 MILiMAC: A Flexible Probe for Delivery and On-site Magnetic Control of Medical Microrobotic Agents

7.1 Introduction . . . . . . . . . . . . . . . . . . . . 132

7.2 The Effect of Scale on Magnetic Actuation Systems . . . . . . . . . . . . . . . . . . . 133

7.3 MILiMAC: Flexible Catheter with Miniaturized Electromagnets . . 137

7.4 Proof-of-Concept Validation . . . . . . . . . . . . . . . . . . 138

7.5 Position Control using MILiMAC . . . . . . . . . . . . . . . . . 139

7.6 Results and Discussion . . . . . . . . . . . . . . . . . . . 142

7.7 Conclusions . . . . . . . . . . . . . . . . . . . . 144

IV Concluding Remarks $\quad 147$

8 Discussion $\quad \mathbf{1 4 9}$

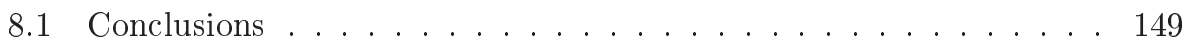

8.1 .1 Part I . . . . . . . . . . . . . . . . 150

8.1 .2 Part II . . . . . . . . . . . . . . . . . . 151

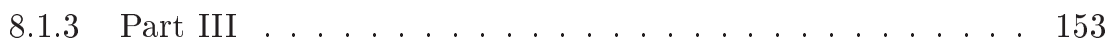

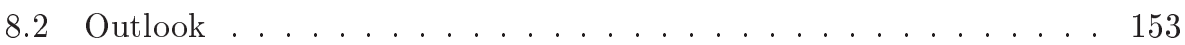

8.2.1 Magnetic Actuation Systems . . . . . . . . . . . . . 154

8.2.2 Catheter Design, Tracking and Control . . . . . . . . . . 155

8.2 .3 Novel Applications . . . . . . . . . . . . . . . . . 156

$\begin{array}{ll}\text { Bibliography } & 158\end{array}$

$\begin{array}{ll}\text { Acknowledgements } & 183\end{array}$

$\begin{array}{ll}\text { About the Author } & 187\end{array}$ 



\section{Chapter 1}

\section{Introduction}

Among scientific disciplines, medicine is unique in that it profoundly impacts some of the most intimate moments of human existence, shaping the story of each human life at its biological foundations. Therefore, each major historical breakthrough in medical practice has had a dramatic, positive impact on humankind, providing means to alleviate countless cases of personal distress, thus changing the attitudes of entire societies towards life. Medical professionals have been laboriously helping us transcend our animal existence, which is still defined by the genetic code we share with our fragile ancestors. The expertise of medical professionals is assisted by a variety of technological developments, such as antiseptics, anaesthesia, antibiotics, vaccination or birth control, used as tools in direct interaction with the form and function of a human being [1-3].

Modern medicine is powerful. A vast majority of those who have the privilege of access to state-of-the-art healthcare can consider the once common phenomena of death and disease as distant and rare [4]. Even during a global SARS-CoV-2 pandemic we are well-equipped as a species. Medical technology has enabled us to identify the threat and to diagnose and support affected individuals. Science provides us with a tangible hope of treatment or eradication of the disease in the foreseeable future. As serious as they are, the biggest challenges imposed by the new virus are socio-economic in nature.

The possibilities offered by modern medicine are enabled by an astonishing amount of progress made in life sciences and engineering over the last few decades. Research and development efforts have translated these accomplishments into technological solutions that empower the practitioners of virtually any branch of contemporary medicine (whom we will refer to as clinicians for the remainder of this dissertation). Examples of groundbreaking advancements are numerous and diverse. Medical imaging technologies revolutionize diagnostics, providing noninvasive methods of inspecting the structure of the body [5]. Pharmaceutical research has improved our capabilities of systemic treatment to the point, where a lethal genetic disease becomes entirely treatable through targeted gene therapy [6]. Information technology (IT) enables large-scale integration of patient-specific information into a coherent database, providing clinicians across institutions with easy access to the entire medical history of an individual [7].

The field of surgery also benefits significantly from this technology-driven evolu- 
tion [8]. Conventional surgical interventions have been relying on acquiring direct and open access to the target site for manual operation. In procedures targeting deeply seated regions of the human body, this usually implies iatrogenic trauma, causing prolonged distress to the patient and generating costs. This motivates the efforts of a large body of clinicians and researchers who seek to reinvent surgical protocols, in order to reduce the invasiveness of treatment. These efforts contribute to the trend of minimally-invasive surgery (MIS) [9].

MIS procedures treat a given condition employing technology to minimize the required operational access. Prominent examples of MIS procedures involve imageguided needle biopsies [10,11], catheter ablation [12,13], radiosurgery $[14,15]$ or endoscopy [16,17]. Clinicians performing MIS rely on technological solutions both for visualisation of the procedure (through miniaturized cameras or medical imaging), as well as for operation (interacting with tissues using agile instruments or ionizing radiation).

Due to restricted visual and operational access to the target site, minimallyinvasive techniques are usually more challenging that open surgery. Therefore, they require substantial expertise from the clinicians, usually achieved by years of training. However, the manual precision of even the best-trained human operator has its boundaries, which limits the potential of MIS in procedures involving deeply seated targets or localised action in small scales. Potential solutions come from the realm of engineering, providing clinicians with instruments that are in essence complete mechatronic systems with dedicated sensing and actuation capabilities. By using automation in these systems, the dexterity of clinicians during MIS can be expanded, potentially opening the possibilities for new target locations and treatment scenarios. These mechatronic instruments - the surgical robots are machines utilising a degree of awareness and autonomy in the execution of mechanical tasks required by a surgical procedure [18]. This dissertation is about a few such instruments, build at the University of Twente (Enschede, the Netherlands) over the course of five years (2015-2020). However, to make their relevance clear, it is useful to begin the story by defining the wider context in which they have come into existence.

\subsection{Robotic Technology in Minimally-Invasive Surgical Procedures}

The first robotic systems to become mature enough for commercial use were articulated robotic arms. These devices were designed to replace industrial workers in manual tasks, such as the positioning of objects in space along assembly lines. This notion motivated initial surgical robotics research, which also sought to use articulated manipulators as a direct substitute for clinicians during manual tasks. These in medical context usually involve tool positioning or tissue extraction.

The application of robotic systems in medical procedures require a reliable three-dimensional models of body tissues geometry. These models should be registered with respect to the pose of the robot, such that it is aware of its motion with respect to the body of patient, in particular the region where a procedure is supposed to take place. These requirements were most readily fulfilled within the 
context of stereotactic surgery, used in MIS procedures that target central nervous system [19]. Thus, a neurosurgical procedure of image-guided needle biopsy of the brain became the first clinical application of robotics [20].

Following this initial success, articulated robots were proposed for autonomous tasks within other medical disciplines [21,22]. Nevertheless, a vast majority of these devices have not produced results justifying their mass-scale clinical development. Autonomous operation inherently involves the challenge associated with the legal responsibility for potential failures of the device. Furthermore, these robots were burdened with both technical and economical issues [18].

A more modern approach to robotic technology in MIS procedures recognizes the inherent strengths of human operators in planning and controlling the execution of manual tasks. Rather than replacing the clinicians entirely, a robotic system can be designed as an advanced surgical instrument for collaborative tasks. Operated by clinicians in real-time through a dedicated interface, such systems are intended to augment human skills at small scales [23]. A survey article by Haidegger et al. provides a detailed overview of various teleoperated surgical robots [24]. The most commercially successful system embodying this approach is certainly the da Vinci Surgical System ${ }^{\text {TM }}$ (Intuitive Surgical, Sunnyvale, CA, USA). (Figure 1.1) First used in 1997 in laparoscopic cholecystectomy in Belgium, it received Food and Drug Administration (FDA) approval in 2000. Since then, Da Vinci ${ }^{\mathrm{TM}}$ became the de facto standard tool for robotic endoscopic surgery [25].

Throughout the years, a large number of clinical studies have demonstrated the superiority of robotic endoscopic surgery performed with the da Vinci ${ }^{\mathrm{TM}}{ }_{\text {sys- }}$ tem over conventional techniques in the treatment of a broad variety of conditions. The distinct ability of the system to allow complex manoeuvres in confined spaces has been reported to facilitate, among others, endoscopic thyroidectomy and nephron-saving partial nephrectomy [26,27]. The reduced procedure time and improved cosmetic effect were reported in the treatment of thyroid and cervical cancers $[28,29]$. Decreased likelihood of conversion to open resection was noted for pancreatectomy [30]. In fact, a systematic review of cost analysis studies indicate that the principal factor preventing wider adoption of this technology is the significantly increased cost of procedures performed with da Vinci ${ }^{\mathrm{TM}}$ in comparison to conventional techniques. [31].

The success of da Vinci ${ }^{\mathrm{TM}}$ in endoscopic applications motivated the intensification of surgical robotics research both in academia and industry. This research aims to find viable robotic technology enabling other type of surgical procedures to be executed in a minimally-invasive fashion [36]. Particular emphasis was put on potential solutions for reducing the invasiveness of procedures performed in challenging locations, such as inside the brain or the heart. The miniaturization limits of articulated arms and the risk of tissue damage they impose, due to their inherent rigidity, render their use in such applications impossible [37]. Thus, the attention of the surgical robotics community focused instead on various groups of manuallyoperated flexible surgical instruments, such as needles, catheters and endoscopes, which are used when minimally-invasive access to a remote target is required.

From an engineering perspective, flexible surgical instruments are continuum devices. Their behaviour differs significantly from the one of industrial robotic arms. Continuum devices (see Figure 1.1) do not bend in discrete locations, but 


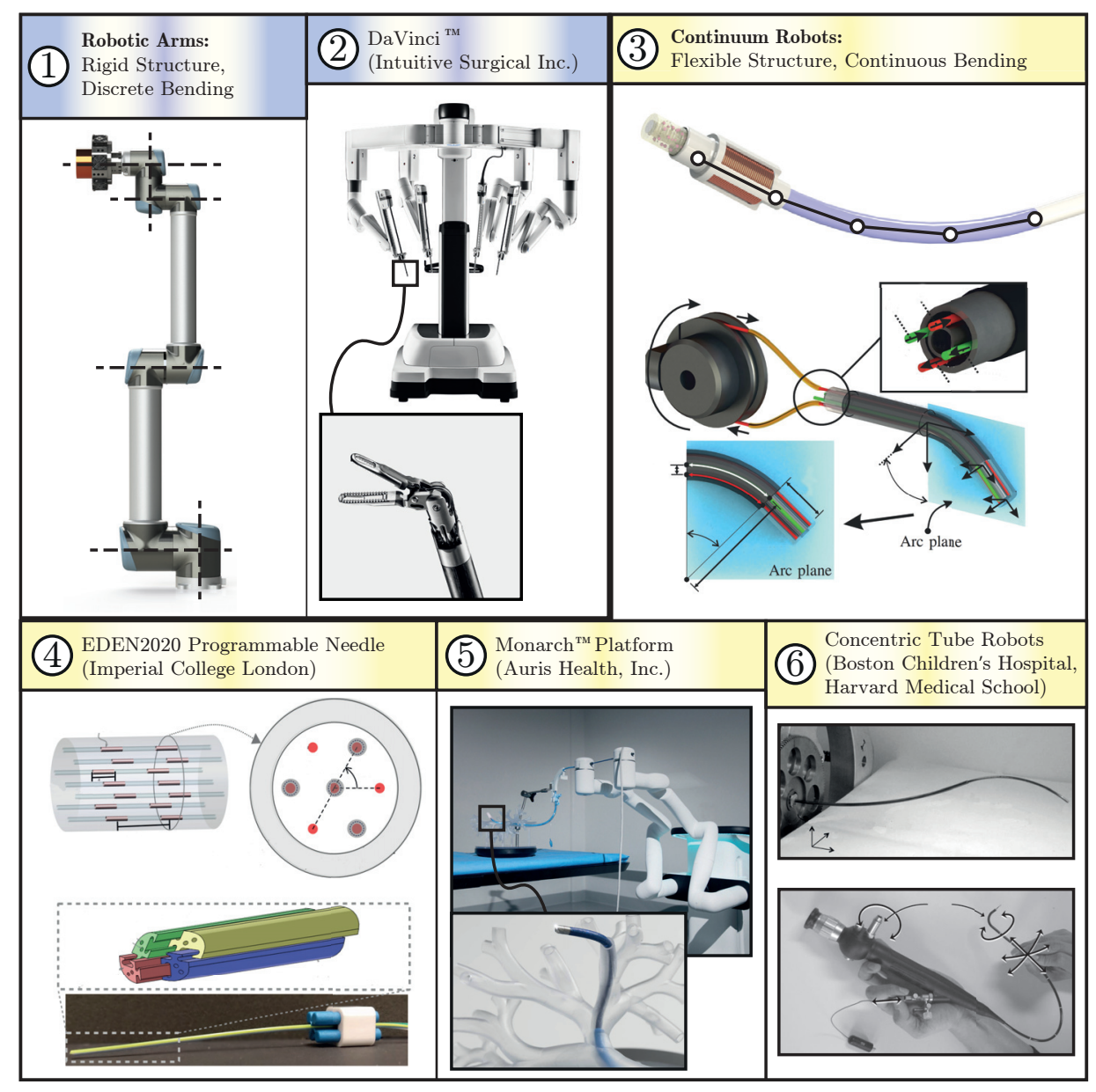

Figure 1.1: The two main classes of robotic devices used in surgical procedures are rigid robotic arms (blue) and continuum robots (yellow). (1) Rigid robotic arms comprise of stiff links connected by discrete motorized joints. The arm bends at the joint locations to generate motion. (2) The most prominent example of a rigid device used for surgical procedures is the da Vinci Surgical System ${ }^{\mathrm{TM}}$ System employing robotic manipulators for teleoperated endoscopic procedures (images courtesy of Intuitive Surgical, Sunnyvale, CA, USA (C) (3) On the other hand, continuum robots use deformation of elastic elements within their entire structure to generate motion. As a result, they exhibit more complex dynamics, which has to be approximated using e.g pseudo-rigid body models or constant curvature approximations (image courtesy of Vrooijink et al. [32] (C)). In addition to catheters, discussed in details in this thesis, other principal types of continuum robots used for surgical procedures involve: (4) flexible needles (image courtesy of Khan et al. [33] (c), (5) steerable endoscopes, among them the Monarch TMPlatfrom (Auris Health, Redwood City, CA, USA), which is a commercial tendon-driven platform for bronchoscopy (images courtesy of Auris Health (c) and (6) concentric tube robots (images courtesy of Dupont et al. and Butler et al. (C)2009 and 2012 IEEE [34,35])

incorporate elastic elements, which continuously change shape in response to mechanical loads [38]. This property allows them to inherently respond to the environmental forces upon contact. The safety of that contact is determined by the 
degree of their compliance matching with the environment [39]. Nevertheless, flexible instruments with compliance closely matching the one of soft tissues experience significant deformation in response to unpredictable external loads present within the surgery site [40]. This fact restricts their precision during manual operation. To remedy that, dedicated sensing and actuation means can be integrated into the structure of flexible surgical instruments to harness their continuum nature. The resulting systems - continuum robots - offer completely new opportunities for surgical procedures realised in a minimally-invasive fashion [41].

\subsection{Catheters as Continuum Robots}

In a clinical context, continuum robotics seeks to control the motion due to deformation of elastic elements within the structure of a given flexible surgical instrument [41]. The technologies exploited for that purpose vary, depending on the particular class of flexible instruments. In this dissertation, we focus on catheters, which are sleek flexible tubes. We recommend the readers who are interested in other classes of robotic flexible surgical instruments to refer to a number of survey articles providing details on technologies specific to flexible needles, active cannulas and endoscopes [41-46].

Catheters find medical uses due to their inherent safety. Their compliance matches the one of soft tissues. This allows the catheters to be inserted into the cavities of the human body with low risk of tissue damage. For manual operation, stiffer catheters are usually preferred due to their increased pushability [47].

Originally, simple catheters with a hollow lumen inside were used for drainage of body liquids or delivery of drugs into the bloodstream [48]. In recent years, more structurally and functionally complex catheters gained wide acceptance as an established class of instruments for MIS [49,50]. Contemporary catheters are invaluable tools to target the cardiovascular (CV) system through small incisions in peripheral arteries, permitting the MIS procedures within the medical fields of interventional cardiology and endovascular surgery [51,52]. These procedures are used with great success to treat thousands of cases of $\mathrm{CV}$ diseases worldwide each year, significantly reducing distress to patients and improving their prognoses.

A prominent example of a successful catheter-based procedure involves a growing number of protocols for endovascular treatment of life-threatening aneurysms. These protocols involve the guidance of catheters through the vascular system to the aneurysm site under fluoroscopic imaging. These catheters are carriers of two groups of deployable elements used to treat the aneurysm: platinum coils, intented to embolise the aneurysm, and endovascular stents, reinforcing the site and preventing it from rupturing $[53,54]$. As these procedures alleviate the need for open surgery, they are associated with better prognoses, especially in older patients [55].

Catheter-based endovascular procedures also gain recognition as viable replacement for certain open-heart surgeries. Most remarkably, catheters revolutionized treatment of myocardial infarctions by enabling angioplasty, which involves unblocking the coronary arteries by catheters containing inflatable cutting-balloons and deployable intravascular stents [56]. These catheters are delivered to the target site through transfemoral or transradial routes [57]. Due to significantly reduced invasiveness, the angioplasty replaced the coronary artery bypass surgery as the 
preferred method of coronary revascularization in a vast majority of cases, thereby becoming one of the most widely applied cardiovascular treatments [58].

The success of currently established catheterization techniques drives the motivation for a wider adoption of this class of instruments within MIS, also outside cardiovascular applications [59-61]. However, performing a procedure with such a flexible instrument is challenging. When the catheter insertion depth increases, clinicians gradually lose the ability to control the motion of catheter tip by operating at the insertion port, due to interaction of the deformable, compliant structure of the catheter with the environment along its entire length within the body [47]. This limitation has been reported to restrict the accessibility of established MIS protocols for patients with challenging anatomy or comorbidities [62-64]. Furthermore, it imposes a hard boundary onto clinicians and medical engineers trying to extend the range of possible MIS procedures executed with manually-operated devices.

Various methods have been devised to give clinicians improved control over the behaviour of the catheter at its tip [65]. Resulting steerable catheters provide more intuitive and less time-consuming insertions and improved dexterity at the target site [66]. These features increase the number of possible endovascular MIS procedures and improve their accessibility. In particular, control of the catheter tip pose allows for procedures such as intracardiac echo (ICE) or cardiac ablation $[67,68]$.

Regardless of the applied actuation technique, manual operation of steerable catheters is still challenging due to limited information available to the clinician regarding the state of the device and its pose with respect to body tissues. Thus, even the execution of standard MIS procedures involving catheters requires clinicians with extensive experience, limiting the accessibility of treatment [69]. The dexterity of steerable catheters is further degraded by presence of unpredictable biological forces [70]. Thus, robotic technology has increasingly been used to enhance the capabilities of clinicians to control steerable catheters, resulting in improved in vivo behaviour $[71,72]$. Additionally, robotically-steered catheters can potentially be used in places where manual operation is considered too dangerous due to delicate structures, such as in neurosurgical procedures. [73].

The largest group of robotically-steered catheters comprises of tendon-driven devices. Tendons run through dedicated channels within the compliant structure of a catheter and transfer tension applied by the actuation unit located outside of the body to the distal section of the catheter [74]. Monarch Platform ${ }^{\mathrm{TM}}$ (formerly known as Sensei Robotic Catheter System; Hansen Medical, Mountain View, CA, USA) is a successful commercial solution for robotic positioning of a tendon-driven endovascular catheter [75]. A growing number of publications positively assesses the performance of this system in a number of classical endovascular applications, such as in cardiac ablation [76-78] or aneurysm treatment [79], identifying improved vascular access and shorter procedure times. A recent review by Peters et al. describes several similar commercial platforms, which are currently under development and have not yet received regulatory approval [80].

In addition to commercial devices, experimental catheters for a wider variety of MIS procedures. Prominent examples include an ultrasound-guided sheath for transapical mitral valve implantation [32, 81], an autonomouslypositioned ICE catheter $[70,82]$, and a minimally-invasive neurosurgical 
intracranial robot (MINIR) [83,84]. The use of tendons for actuation is popular not only in steerable catheters, but across entire continuum robotics, as this method of actuation is relatively simple to implement and performs with sufficient reliability in a large range of applications [85].

Despite the popularity of tendon-driven actuation, its operational performance in applications as critical as catheter steering during MIS procedures is sufficient predominantly for positioning tasks. As tendons move during catheter deflection, they are subject to friction, which prevents effective force coupling between the catheter base and the tendon attachment point, usually close to the tip of the device [86]. This degrades the reliability of actuation and generates unwanted behaviour in the dynamics of tendon-driven catheters [87]. This behaviour involves phenomena such as backlash and hysteresis which have to be compensated for, which is challenging. The effects of friction become more prominent in smaller scales or at large bends, which sets a limit on miniaturization potential of tendondriven catheters and on their overall dexterity [41].

A variety of alternative actuation techniques have been explored to create prototypes of robotically-steered catheters that expand the boundaries of attainable precision and open up new possible clinical applications. Prominent examples involve hydraulics/pneumatics [88-90], thermal actuation [91,92], and the use of parallel concentric tube elements [93]. Detailed surveys on that topic are presented in $[65,94]$. In this dissertation we extensively study one such technique - the magnetic actuation. We design novel robotically-steered catheters actuated in non-contact fashion by exploiting magnetic interaction between a number of elements belonging to one of two categories: the permanent magnets or electromagnetic coils.

Magnetic catheters have simple structure with no moving components, which allows for substantial miniaturization [95]. They are actuated through a process, which can be modelled precisely and efficiently [96]. Their complex mechanical behaviour gives them potential dexterity suitable for a wide range of applications [97]. By exploring the domain of magnetic actuation, we intend to exploit these advantages, helping these devices to become an established class of instruments for MIS procedures.

\subsection{Magnetic Actuation}

The technique of magnetic actuation can be applied to any object with magnetic properties. When such objects interact with a magnetic field, they experience a wrench [98]. This interaction is complex and occurs at molecular scale. However, in robotic applications, it can usually be modelled analytically by using efficient approximations based on linear algebra [99].

These approximations assume that the object with magnetic properties occupies a point-location in space. The sum of these properties across the entire non-deformable volume of that object is represented by a single vector quantity: the magnetic dipole moment $\left(\boldsymbol{\mu} \in \mathbb{R}^{3}\right)$ [100]. By generating an external field, represented as a vector $\left(\mathbf{B} \in \mathbb{R}^{3}\right)$, at the location of the object, magnetic force 


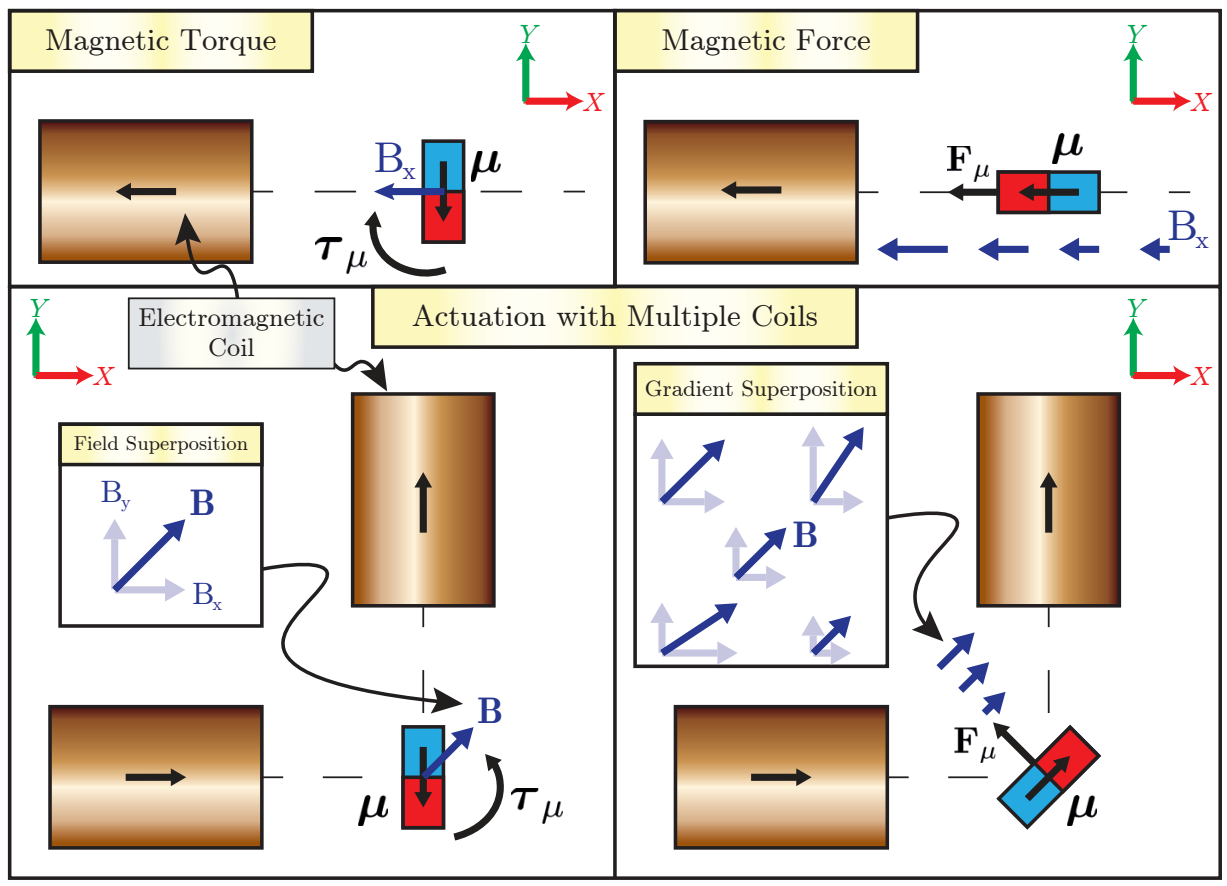

Figure 1.2: Any object with magnetic properties, represented by a magnetic dipole moment $\left(\boldsymbol{\mu} \in \mathbb{R}^{3}\right)$, experiences a force $\left(\mathbf{F}_{\mu} \in \mathbb{R}^{3}\right)$ and torque $\left(\boldsymbol{\tau}_{t} \in \mathbb{R}^{3}\right)$ when placed in external magnetic field $\left(\mathbf{B}=\left[\begin{array}{lll}B_{x} & B_{y} & B_{z}\end{array}\right]^{T} \in \mathbb{R}^{3}\right)$. Magnetic torque compels the object to rotate, such that the direction of its $\boldsymbol{\mu}$ aligns with $\mathbf{B}$. The value of magnetic force is determined by the product of $\boldsymbol{\mu}$ and the spatial gradient of the external field $\left(\nabla \mathbf{B} \in \mathbb{R}^{3 \times 3}\right)$. As both magnetic fields and gradients superpose at any location, multiple magnetic field sources can be used to determine the total field and spatial gradient at a location of the object. This way, prescribed forces and torques can be exerted on the actuated object. This allows for control of both the position and the orientation of an object in a non-contact manner.

$\left(\mathbf{F}_{\mu} \in \mathbb{R}^{3}\right)$ and magnetic torque $\left(\boldsymbol{\tau}_{t} \in \mathbb{R}^{3}\right)$ are exerted on the object as follows:

$$
\mathbf{W}=\left[\begin{array}{c}
\mathbf{F}_{\mu} \\
\boldsymbol{\tau}_{\mu}
\end{array}\right]=\left[\begin{array}{c}
\nabla(\mathbf{B} \bullet \boldsymbol{\mu}) \\
\boldsymbol{\mu} \times \mathbf{B}
\end{array}\right],
$$

where nabla $(\nabla)$ is a gradient operator [101]. Figure 1.2 provides an illustrative explanation of this phenomenon.

Magnetic interaction is versatile, and can be applied for actuation in a variety of fashions, depending on the structure and desired application of the actuated system [102]. It has gained particular attention in medical robotics due to its noncontact nature. This nature allows to keep the infrastructure used for magnetic actuation - usually comprising of large permanent magnets or electromagnetic coils - outside the body of the patient. In the same time, the actuation forces and torques can be applied directly at the desired point within the structure of a robot. A large body of research uses magnetic actuation for motion control of a various microrobotic agents [103-105]. Most of the classical designs of infrastructure for 
the generation of external magnetic fields, such as the pioneering OctoMag, come from that field [106-108]. Research on magnetic needles and endoscopes has also produced remarkable results, indicating a potential for clinically-applicable solutions [109-113].

The idea of using magnetic interaction to actuate a flexible catheter is not new [114]. The most conventional realisation of this method involves integrating permanent magnets close to the tip of the device. By using a source of varible external magnetic field (B), such a catheter can be deflected in a given direction [115-118]. Another prominent types of magnetic catheters are designed to work in static external field of the MR scanner. MR-compatible catheters employ electromagnetic coils with different geometries integrated onto its body. Directional motion is achieved by activating a particular combination of coils to generate a variable dipole moment $(\boldsymbol{\mu})$, dependent on the orientation of the catheter with respect to the external field [119-122].

A few commercial solutions involving magnetically-actuated catheters have been presented across the last two decades. The most successful of them, and the only one to reach the market, is Niobe ${ }^{\mathrm{TM}}$ (Stereotaxis, St. Louis, MO, USA) [123]. Niobe ${ }^{\mathrm{TM}}$ is a system for remote guidance of magnetic catheters during cardiac ablation by utilising two large electromagnets to generate an external field deflecting the catheter tip [124]. The direction of the field is controlled directly by the clinician, who monitors the position of the catheter using fluoroscopy. A recent meta-analysis has demonstrated distinct advantages of Niobe ${ }^{\mathrm{TM}}$ in cardiac ablation, reporting almost $50 \%$ lower risk of complications with respect to standard catheterization [125]. Furthermore, the reduced size and improved dexterity of the catheters actuated by Niobe ${ }^{\mathrm{TM}}$ makes them a viable solution for challenging coronary interventions in small and torturous vessels $[126,127]$. However, another studies indicate that procedures performed using Niobe ${ }^{\mathrm{TM}}$ suffer from significantly longer execution time than conventional methods $[128,129]$.

Recently, an improved version of the Niobe ${ }^{\mathrm{TM}}$ system, named Genesis ${ }^{\mathrm{TM}}$, has been proposed by Stereotaxis, and has received FDA approval for clinical use [130]. While based on the same principle, Genesis ${ }^{\mathrm{TM}}$ has smaller footprint and increased magnet motion range. Additionally, several other clinically-applicable devices for the steering of magnetic catheters have been devised, but their development has been suspended or remains in the pre-commercial stage [131-133].

\subsection{Research Objectives and Outline of the Thesis}

Despite the progress of the last decade, the field of magnetic catheters is still new and exciting. Catheters usually have diameters of a few millimetres. Thus, they reside in the mesoscale, at the boundary of macroscopic and microscopic worlds. Because of that, they offer a set of unique clinical possibilities (with the most obvious one being the capability to navigate through cavities of human body). However, due to their size, their development is also subjected to a variety of research challenges belonging both the macro- and micro-domain. These challenges are related to the fabrication and handling of magnetic catheters as well as to the robotic technology required for their successful operation. 
Current state-of-the-art research uses catheters that are relatively simple in structure and are targeted at a handful of popular endovascular applications, such as cardiac ablation or angioplasty $[134,135]$. This certainly does not fully exhaust the subject. Significant potential exists to produce novel magnetic catheter designs capable of complex behaviour and thus facilitate advanced, clinically-relevant functionalities. This is the principal research direction we undertake in this dissertation.

The broad context of surgical robotics provides an established methodology to rely on, helping us push the research frontier within the subject of magnetic catheters forward, closer to clinical practice, as well as sideways, towards novel applications. After an extensive literature search, several key research questions have been identified as the principal driving force behind the work done in this dissertation. Ultimately, the following research questions have been formulated:

RQ.1 How to make the auxiliary infrastructure for magnetic actuation more clinically-relevant?

Magnetic catheters require reliable auxiliary infrastructure, capable of generating magnetic field in a clinically-relevant workspace. This requirement can be approached in a multitude of fashions, but successful solutions are non-trivial to devise due to the unfavourable scaling properties of magnetic fields. For that reason, the systems designed for workspaces encompassing the entire human body (including the commercial Niobe ${ }^{\mathrm{TM}}$ ) are large and bulky, have high power demands, and offer relatively low actuation bandwidth $[116,124,134]$. As an alternative, systems with smaller workspaces are commonly used, usually employing stationary electromagnetic coils [107]. This trend has been initiated by the OctoMag system, originally intended for the actuation of micro-robotic agents in eye surgery applications, and since then used also in a handful of studies on magnetic catheters [106, 136,137]. Another method, developed in part concurrently with the research presented within this dissertation, uses mobile permanent magnets to guide catheters and endoscopes [110,138-140]. In this dissertation, we present a line of research on using mobile electromagnetic coils as auxiliary infrastructure. We argue that by using mobile coils, actuation in enlarged, clinically-relevant workspaces can be achieved, while ensuring the safety of operation, and providing strong magnetic actuation.

\section{RQ.2 Which techniques can be used to build functionalised magnetic catheters for novel applications?}

Conventional research on magnetic catheters aims at increasing precision during conventional MIS procedures [116,136, 141-143]. Additionally, several functionalised magnetic catheters have been proposed predominantly for tasks involving removal of tissue using incisions or ablation [95, 144, 145]. These relatively modest applications neither exhaust the potential of magnetic catheters, nor provide convincing justification for regular clinical use of them, as the complex auxiliary infrastructure required involves large capital costs. The advances in the domain of untethered milli-/microrobotics indicate that magnetic actuation can be used to create significantly more advanced devices $[146,147]$. The rapidly evolving field of soft robotics has 
also demonstrated that complex mechanical behaviour of flexible elements can be achieved by smart use of magnetic elements [148-151]. By exploring the subject of the design of magnetic catheters, we aim to create devices for clinically-relevant tasks within the human body. We demonstrate that catheters with advanced functionalities can be devised using various magnetic elements. With these developments, we hope to expand the potential of magnetic actuation in MIS procedures of the future, paving the way through which magnetic catheters can find their application in a diverse range of treatment protocols.

\section{RQ.3 How to enable vision-based 3D tracking of the entire shape of a magnetic catheter?}

Reliable information about the state of a catheter during an MIS procedure is crucial both for the clinicians involved, as well as for the controllers employed within the associated robotic system. Medical imaging modalities, such as fluoroscopy or ultrasound, are commonly used for that purpose, as they allow for visual tracking of flexible instruments within the global context of its environment $[152,153]$. In the literature, the techniques applied to steerable catheters usually focus on recovering tip position, which is not sufficient for reliable real-time modelling of the entire continuum device $[32,116,154,155]$. Multiple general techniques have been developed for $3 \mathrm{D}$ shape reconstruction of various continuum robots using visual input; nevertheless, none of them has been applied within the context of magnetic catheters [156-158, 158-160]. In this work, we create a vision-based framework which allows for fast and reliable reconstruction of the full 3D configuration of a magnetic catheter, intended for use with medical imaging modalities. We synthesise a modular shape sensing algorithm using a mix of well-established machine vision methods and novel techniques. We validate our algorithm experimentally, initially with a stereo vision system employing optical cameras, and ultimately demonstrating its clinical relevance by incorporating it within a custom-build scanning ultrasound device.

RQ.4 How the existing techniques for modelling and control of continuum robots can be applied to novel magnetic catheters?

The mechanical behaviour of continuum robots is governed by partial differential equations which are computationally intensive to solve [161]. Thus, for real-time applications, a large number of different approximate models have been proposed, with varying levels of complexity and accuracy [41]. Two main approaches are present in the literature. The first one employs the Cosserat rod theory to create models that retain the phenomenon of continuous deformation [162-164]. The second approach takes the approximation of continuum robots one step further, representing it as a series of discrete links connected by compliant joints [165-167]. The resulting pseudo-rigidbody (PRB) representation is simple, computationally-efficient, and allows for applications of mathematical techniques developed for classical industrial robots. Research concurrent to this dissertation has demonstrated that both models can be used to derive position controllers for various types of 
magnetic catheters $[116,141]$. Due to a large body of pre-existing theory, with limited experimental treatment, our work on modelling and control of magnetic catheters focuses on integrative aspects. We employ the PRB modelling approach based on works of Greigarn and Çavuşoğlu [121,168,169] within the context of our work on vision-based tracking. We demonstrate that such a model can be effectively reconstructed in real-time from catheter shape data. Subsequently, we use this PRB model along with mathematical representation of our auxiliary infrastructure to derive controllers driving the operation of our novel magnetic catheters during clinically-relevant tasks.

These general questions were used to formulate a broad range of hypotheses touching various aspects of robotics. Whereas the research questions remained steady, these hypotheses were often prone to change under the influence of new findings and publications of concurrent work by other authors. The specific hypotheses, which were proven correct and have successfully undergone the peerreview process, are introduced and discussed against specific literature within the chapters of Parts I-III of this thesis.

The thesis begins with Part I, where we address the research question RQ.1. It gives account of the development process of two generations of auxiliary infrastructure for magnetic actuation. The first generation system - BigMag - is presented in Chapter 2. It is an array of six mobile electromagnetic coils, driven by an optimization-based inverse field map, and can generate prescribed threedimensional magnetic fields in any direction. The know-how established during the development and testing of BigMag serves to inform a second generation infrastructure, the ARMM (Advanced Robotics for Magnetic Manipulation) system. This system is described in Chapter 3. It uses a single mobile coil located on a 6 degrees-of-freedom (DoF) robotic arm and can provide magnetic fields in workspace sufficient for clinical use.

The remainder of the scientific work within the dissertation focuses on development of various magnetic catheters for MIS procedures. In Part II we use our auxiliary infrastructure in three studies, each one involving a novel magnetic catheter for a different clinical application. Chapter 4 describes integrative work, in which we develop a complete framework for $3 \mathrm{D}$ position control of a magnetic catheter using BigMag. The framework employs a pseudo-rigid-body model to derive an reduced-order inverse model controller of a catheter. In this chapter, we also present the foundations for our vision-based tracking method. Chapter 5 demonstrates our first functionalised catheter, using two magnetic elements to turn the flexible structure of a catheter into a steerable tentacle for underactuated grasping. Chapter 6 revisits the techniques developed earlier within this part, applying them to a novel catheter realising the task of targeted drug delivery. In this chapter, our modelling and control framework is expanded to allow for steering of this catheter using the ARMM system. Furthermore, we also demonstrate the clinical relevance of our tracking method, which is used to extract information about the configuration of the catheter from images acquired with custom-built scanning ultrasound machine.

Part III approaches the topic of magnetic catheters from a different perspective, combining the methods presented both in Part I and Part II. In Chapter 7, we propose MILiMAC (Microrobotic Infrastructure Loaded into Magnetically- 
Actuated Catheter), which is both a magnetic catheter and auxillary infrastructure in one. The task for which MILiMAC has been designed involves localised control of microrobotic agents within the confines of the human body. In this chapter, we demonstrate, how magnetic catheters can evolve into complex intermediaries enabling the clinicians, who operate in macroscale, to use devices designed for procedures at cellular and tissue scales, thereby opening completely new niches within the domain of minimally-invasive surgery.

\subsection{Scientific Output}

The project described in this dissertation has been generously funded by the European Research Council (ERC) under the European Union's Horizon 2020 Research and Innovation programme (Grant Agreement \#638428 - project ROBOTAR). The studies realised as a part of this dissertation have also been presented in a series of scientific publications. Additionally, the expertise developed within the research line presented in this dissertation have been transferred to a number of another projects, which amplified the total research output by several additional items. Across all chapters, the content of this thesis relates to the context set by the following scientific publications:

\section{Peer-Reviewed Journal Articles}

- C.M. Heunis, J. Sikorski, S. Misra. Flexible instruments for endovascular enterventions, IEEE Robotics and Automation Magazine vol. 25, no. 3, pp. 71-82, 2018

- J. Sikorski, A. Denasi, G. Bucchi, S. Scheggi, S Misra. Vision-based 3-D control of magnetically actuated catheter using BigMag - an array of mobile electromagnetic coils, IEEE/ASME Transactions on Mechatronics vol. 24, no. 2, pp. 505-516, 2019

- J. Sikorski, C.M. Heunis, F. Franco, S. Misra. The ARMM system: An optimized mobile electromagnetic coil for non-linear actuation of flexible surgical instruments, IEEE Transactions on Magnetics vol. 55, no. 9, pp. 1-9, 2019

- V. Kalpathy Venkiteswaran, L.F.P. Samaniego, J. Sikorski, S. Misra. Bioinspired terrestrial motion of magnetic soft millirobots, IEEE Robotics and Automation Letters vol. 4, no. 2, pp. 1753-1759, 2019

- V. Kalpathy Venkiteswaran, J. Sikorski, S. Misra. Shape and contact force estimation of continuum manipulators using pseudo rigid body models, Mechanism and Machine Theory vol. 139, pp. 34-45, 2019

- C.M. Heunis, Y. Wotte, J. Sikorski, G. Phillips Furtado, S Misra. The ARMM system - autonomous steering of magnetically-actuated catheters: towards endovascular applications, IEEE Robotics and Automation Letters vol. 5, no. 2, pp. 705-712, 2020 
- J. Sikorski, S. Mohanty, S. Misra. MILiMAC: Flexible catheter with miniaturized electromagnets as a small-footprint system for microrobotic tasks, IEEE Robotics and Automation Letters vol. 5, no. 4, pp. 5260 - 5267, 2020

- J. Sikorski, C.M. Heunis, R. Obeid, V. Kalpathy Venkiteswaran, S. Misra. Flexible Catheter for Ultrasound-Guided Delivery of Magnetic Projectiles, IEEE Transactions on Robotics [Submitted]

\section{International Peer-Reviewed Conferences}

- I.R. Dawson, F. Campisano, F. Gramuglia, K.L. Obstein, E.E.G. Hekman, J. Sikorski, S. Misra, P. Valdastri. Modeling and validation of a low-cost soft-tethered endoscopic platform, Proceedings of the 9th Hamlyn Symposium on Medical Robotics 2016: New Challenges and Emerging Platforms, London, UK, 2016

- J. Sikorski, I.R. Dawson, A. Denasi, E.E.G. Hekman, S. Misra. Introducing BigMag - A novel system for 3D magnetic actuation of flexible surgical manipulators, Proceedings of the 2017 IEEE International Conference on Robotics and Automation (ICRA), Singapore, pp. 3594-3599, May-June 2017

- J. Sikorski, E.S.A.A.M. Rutting, S. Misra. Grasping using magneticallyactuated tentacle catheter: a proof-of-concept study, Proceedings of the 7th IEEE RAS/EMBS International Conference on Biomedical Robotics and Biomechatronics (BIOROB 2018), Enschede, The Netherlands, pp. 609-614, August 2018

- C.M. Heunis, J. Sikorski, G. Phillips Furtado, S Misra. The ARMM system: Demonstrating clinical feasibility in steering magnetically actuated catheters in endovascular applications, Proceedings of the 2019 IEEE/RSJ International Conference on Intelligent Robots and Systems (IROS), Macau, China, November 2019

- J. Sikorski, S. Mohanty, S. Misra. MILiMAC: Flexible catheter with miniaturized electromagnets as a small-footprint system for microrobotic tasks, Proceedings of the 2020 IEEE International Conference on Intelligent Robots and Systems (IROS), Las Vegas, NV, USA, October 2020 [Accepted] 


\section{Part I}

\section{Starting Big: Magnetic Actuation Systems}





\section{Preface}

Development of a system for magnetic steering of catheters is a task with involved nature. Such systems rely on the interaction of two components with vastly different sizes and dynamical behaviour: the magnetic catheter itself and the infrastructure generating the external magnetic field. The desired structure and behaviour of either of these components is dependent on the other. The limitations imposed by infrastructure constrain the possible catheter designs. Conversely, while designing a magnetic catheter one has to take into consideration the external magnetic fields and gradients necessary to actuate it. At the start of the design process, a decision must be made on how to untangle this interdependence in a meaningful way. As our ultimate goal is to explore novel designs of magnetic catheters with potentially very different external field requirements, versatility of the auxiliary infrastructure is a key factor for the success of the entire project. Hence, we first focus on means to generate external magnetic field. We explore the concept of mobile electromagnets, allowing us to build systems adaptable to a wide range of potential catheter designs.

We start big, literally. The first result of our work, BigMag is an array of six mobile electromagnets located around a spherical workspace roughly comparable in size to human heart. In Chapter 2 we describe its development process and composition. Yet more importantly, we present the techniques for forward and inverse modelling of this class of devices. These techniques allow for autonomous control of the magnetic field inside the workspace of BigMag. The resulting system provides high-bandwidth three-dimensional magnetic actuation with the fields of at least $40 \mathrm{mT}$, suitable for actuation of a wide range of magnetic catheters.

As an electromagnetic coil array BigMag remains burdened by scalability issues preventing its conversion into a clinically-relevant system. Nevertheless, the underlying paradigm of using mobile electromagnets does have a potential of generating systems capable of clinical use. We demonstrate this in Chapter 3, where the knowhow generated during BigMag development is used to create second-generation electromagnetic actuator to be used within the ARMM (Advanced Robotics for Magnetic Manipulation) system. This actuator comprises of an optimized electromagnetic coil positioned in space by 6 DoF robotic arm, allowing for a spherical reachable workspace with a diameter of $2.6 \mathrm{~m}$. This enlarged workspace is considered suitable for clinical operation. An iterative inverse map technique is used to realise independent magnetic field/gradient control using the actuator, generating the fields exceeding $20 \mathrm{mT}$ and gradients of up to $0.6 \mathrm{~T} / \mathrm{m}$. Feedback from a magnetic field sensor is used for precise control in the saturation region of the coil. 



\title{
Chapter 2
}

\section{BigMag: A Novel System for 3D Magnetic Actuation of Flexible Surgical Manipulators}

\begin{abstract}
Magnetic interaction can be utilized for remote actuation of surgical manipulators. However, platforms currently available for that purpose have limited workspaces, inadequate field strength or very low bandwidth of the electrical subsystem. In this chapter, we present BigMag, a novel platform capable of magnetic steering of continuum manipulators for medical purposes. BigMag comprises of 6 mobile coils and is capable of generating the fields of at least $40 \mathrm{mT}$ in any direction at every point of its workspace. Moreover, we introduce a mathematical model for 3D mobile coil arrays. Magnetic actuation is enabled using an inverse field map technique, mapping the reference magnetic field to BigMag configuration variables. The inverse map is validated, showing a mean magnetic field error of $2.20 \%$. The investigation concludes with a demonstration of BigMag capabilities in actuation of magnetic catheters in confined spaces using user-controlled steering.
\end{abstract}

Adapted from:

J. Sikorski, I.R. Dawson, A. Denasi, E.E.G. Hekman, S. Misra. Introducing BigMag - A novel system for 3D magnetic actuation of flexible surgical manipulators, Proceedings of the 2017 IEEE International Conference on Robotics and Automation (ICRA), Singapore, pp. 3594-3599, May-June 2017

with the inverse field map adapted from:

J. Sikorski, A. Denasi, G. Bucchi, S. Scheggi, S Misra. Vision-based 3-D control of magnetically actuated catheter using BigMag - an array of mobile electromagnetic coils, IEEE/ASME Transactions on Mechatronics vol. 24, no. 2, pp. 505-516, 2019 


\subsection{Introduction}

Robotic systems have the potential to revolutionize minimally invasive surgical procedures [18]. One group of devices particularly explored for applications within that field are continuum manipulators [41]. They differ from classical rigid-link manipulators by having a hyper-redundant structure of continuously bending, elastic elements [170]. They are well-suited for medical procedures, due to their inherent compliance. This property allows them for safe operation in confined cavities of the human body, even if unwanted contact with tissue was to occur [93]. In particular, numerous attempts have been made to use continuum manipulators to create robotic endovascular catheters [65].

The challenge of developing reliable remote actuation for continuum manipulators has been addressed in multiple works. The most conventional approach involves displacing tendons [81,171], or elastic tubes [162]. However, the moving parts experience significant friction, making the steering imprecise and subjected to hysteresis, and calling for an alternative [172].

Since the introduction of low-frequency magnetic steering of surgical devices the subject has gained significant attention [114]. The strength and reliability of magnetic interaction are sufficient for actuation of continuum manipulators inserted into the human body. Moreover, magnetic fields pose no radiation hazard and thus they may be used even for lengthy surgical procedures [173].

Several custom devices for magnetic actuation of continuum manipulators have been presented in the literature. OctoMag is an array of coils with fixed positions originally used for microrobotics research [106]. Ullrich et al. used it to steer a magnetic microcatheter [174]. The main limitation of OctoMag is its operational workspace, which narrows its clinical relevance down to micro-scale manipulators. This problem was partially addressed by Le et al., who introduced an array with bigger workspace [117]. Nevertheless, the field model presented therein has not been directly validated. This fact limits the applicability of that system in automated steering, as the wrenches acting on the manipulator cannot be estimated precisely.

Another problem shared by the aforementioned systems is their limited scalability. Enlarging the static coils, used in those devices, increases their inductance, hindering the effective magnetic field control. Niobe ${ }^{\circledR}$ (Stereotaxis Inc., St. Louis, USA) employs permanent magnets instead of coils to address this problem [175]. However, this method of generating magnetic fields raises safety concerns, as the system cannot be switched off. Moreover, its magnetic field is controlled only by dislocation of the magnets, which slows down the operation of the system. Thus, Niobe ${ }^{\circledR}$ has limited capabilities in applications requiring fast actuation. Therefore, it is unable to compensate for tissue motion, or be used in force control.

In our preceding work, we demonstrated that a magnetic catheter can be steered in 2D using an array of stationary Helmholtz coils under ultrasound guidance [155]. In this chapter we introduce and characterize BigMag. It is a system using six mobile coils for 3D magnetic actuation of continuum manipulators (Figure 1). We also propose and validate a model of the magnetic field inside the functional workspace of BigMag. Such a model is required to apply known magnetic wrenches to continuum manipulators. In contrast to most existing devices, 
the coils of BigMag rotate around its workspace. The advantages of such an approach have been already discussed in the literature [176]. To the authors' best knowledge, BigMag is the first device using mobile coils for 3D steering of continuum manipulators.

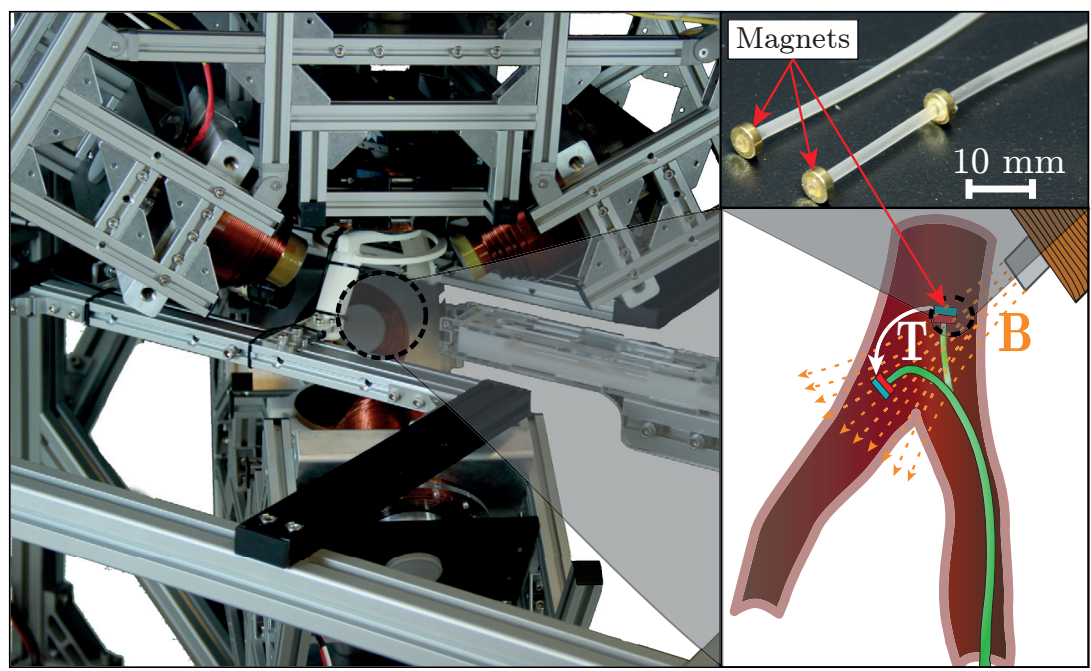

Figure 2.1: Automation of flexible surgical manipulators requires reliable actuation. Such actuation is possible by equipping the manipulators with permanent magnets, which interact with external magnetic field (B). Torque (T), produced as a result of this interaction, deflects the manipulator. This chapter presents BigMag - a device using an array of mobile coils to generate arbitrary magnetic fields inside clinically-relevant spaces. Those fields are strong enough to achieve 3D steering of continuum manipulators. Left: functional workspace of BigMag - a sphere with a diameter of $100 \mathrm{~mm}$. Right top: examples of relevant manipulators, equipped with magnets. Right bottom: a target clinical scenario - steering of a flexible manipulator inside the vasculature.

The rest of the Chapter is structured as follows: Section 2.2 introduces BigMag and explains its design rationale. Section 2.3 presents a mathematical model for the magnetic fields, which is used to describe BigMag. Subsequent validation of the model is presented in Section 2.4, along with a demonstration of BigMag being used in manual steering of magnetic catheters. The chapter is concluded by Section 2.5 presenting the directions for future work.

\subsection{Design of BigMag}

BigMag has been designed through an iterative process in an attempt to optimize most of its parameters. The goal of the investigation is to meet the field requirements for the selected workspace. This section describes the rationale behind that process. It also presents the architecture of the BigMag with all its subsystems, and key parameters (see Figure 2.2). 


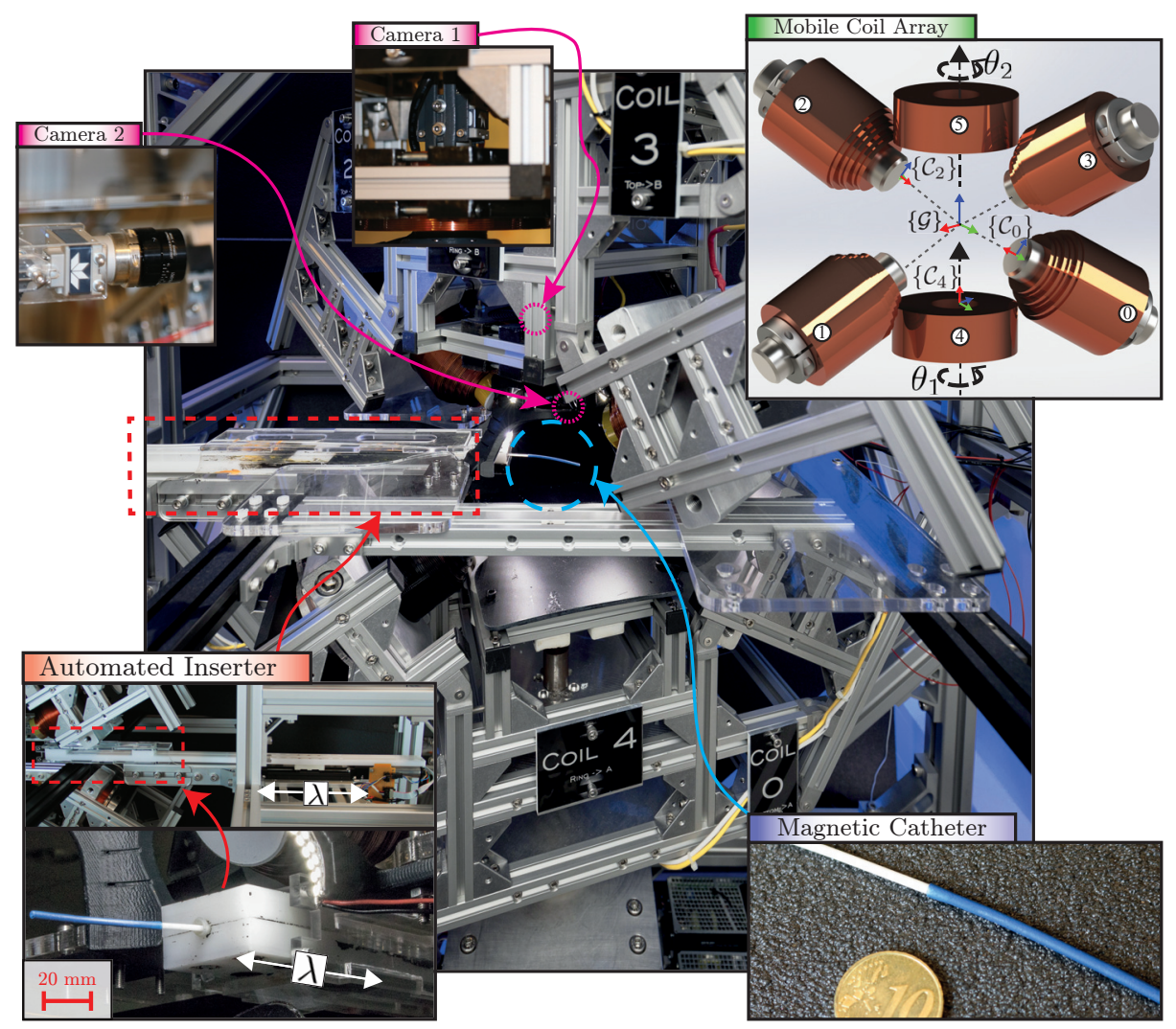

Figure 2.2: BigMag is an array of six mobile coils, capable of providing actuation of magnetic catheters in a spherical workspace with a diameter of $100 \mathrm{~mm}$ (blue circle). Moreover, BigMag is equipped with an automated inserter (red rectangle) controlling the insertion position of the magnetic catheter $(\lambda)$, and two cameras used for stereo vision. The coils of BigMag are located on two independently rotating fixtures around the $\mathrm{Z}$ axis of global reference frame $(\{\mathcal{G}\})$. Each coil $(k)$ generates magnetic field expressed in a local reference frame $\left(\mathcal{C}_{k}\right)$. The position of fixture holding coils $(0,1,4)$ is described by angle $\left(\theta_{1}\right)$, whereas $\theta_{2}$ denotes the position of fixture for coils $(2,3,5)$. The axes in all reference frames are red, green and blue for $X, Y$ and $Z$ respectively.

\subsubsection{Design Rationale}

In order to establish the requirements for the system, let us consider a continuum manipulator with magnets attached to its body. The strength of a magnet located at position $\left(\mathbf{p}_{\mathbf{n}} \in \mathbb{R}^{3}\right)$ is defined by its magnetic dipole moment $\left(\mathbf{m} \in \mathbb{R}^{3}\right)$. This dipole interacts with the external magnetic field $\left(\mathbf{B}_{\mathcal{G}}\left(\mathbf{p}_{\mathbf{n}}\right) \in \mathbb{R}^{3}\right)$, experiencing a wrench $\left(\mathbf{W} \in \mathbb{R}^{6}\right)$, comprising of force $\left(\mathbf{F} \in \mathbb{R}^{3}\right)$ and torque $\left(\mathbf{T} \in \mathbb{R}^{3}\right)$ as described by $(1.1)$.

The generation of spatial gradients $\left(\nabla \mathbf{B}_{\mathcal{G}} \in \mathbb{R}^{3 \times 3}\right)$ to steer a continuum manipulator was reported infeasible, even for smaller workspaces $[174,177]$. It is challenging to achieve forces of adequate magnitudes. Therefore, the application of torque ( $\mathbf{T}$ ) has been selected as the primary actuation method of continuum manipulators. Following that decision, the design requirements for the system 
Table 2.1: Design Requirements for BigMag

\begin{tabular}{ll}
\hline Requirement & Motivation \\
\hline $\begin{array}{l}\text { Spherical effective workspace with a } \\
\text { diameter of } 100 \mathrm{~mm}\end{array}$ & $\begin{array}{l}\text { This volume corresponds to a human } \\
\text { heart. It is considered sufficient for } \\
\text { ex vivo trials on biological tissue [178] }\end{array}$ \\
\hline $\begin{array}{l}\text { Generation of fields of at least } 40 \mathrm{mT} \\
\text { in arbitrary 3D direction at any } \\
\text { point inside the workspace. }\end{array}$ & $\begin{array}{l}\text { Based on previous research, fields of } \\
40 \mathrm{mT} \text { were considered sufficient for } \\
\text { effective actuation of flexible } \\
\text { catheters [155]. }\end{array}$ \\
\hline $\begin{array}{l}\text { Magnetic actuation of microrobotic } \\
\text { agents. }\end{array}$ & $\begin{array}{l}\text { Such capability increases the range of } \\
\text { possible applications of the system. }\end{array}$ \\
\hline $\begin{array}{l}\text { Automated insertion of continuum } \\
\text { manipulators inside the workspace }\end{array}$ & $\begin{array}{l}\text { This allows for simulation of } \\
\text { clinically-relevant steering tasks. }\end{array}$ \\
\hline
\end{tabular}

have been established, and are presented in Table 2.1.

BigMag is the realisation of the system following these criteria. It comprises of six coils (Table 2.2), held in two symmetrical frames. Three coils are suspended above the horizontal centre plane of the workspace, the other three are located below it. Such an arrangement is proposed in order to the make the workspace accessible through its centre plane with $360^{\circ}$ clearance.

\subsubsection{BigMag - General Overview}

The two mobile frames of BigMag are actuated by Maxon EC60 series BLDC motors (Maxon Motors, Sachseln, Switzerland). They rotate independently along a common, vertical axis at speeds up to 30 RPM. The motors are controlled by iPOS4808 BX-CAT (Technosoft Motion, Neuchatel, Switzerland) motion controllers. The current in each coil is controlled using Copley XE-230-20 amplifiers (Copley Controls, Canton, USA)

BigMag hosts 2 Dalsa Genie Nano-C1940 (Waterloo, Ontario, Canada) cameras, allowing for visualization of the entire workspace. Moreover, the system is equipped with an automated advancer. It allows for insertion of continuum manipulators with diameters between 1 and $5 \mathrm{~mm}$. The advancer is steered by a linear actuation unit, comprising of a LX20 linear stage (Misumi Europa GmbH, Schwalbach am Taunus, Germany) powered by a Maxon EC22 motor, and controlled by an iPOS4808 BX-CAT motion controller.

Stepped coils of BigMag are equipped with soft iron cores (Sekels GmbH, OberMoerlen, Germany) to increase the field strength. The ring-shaped coils ( 5 and 6 in Figure 2.3) do not contain iron cores. This allows for actuation along the vertical axis with much higher bandwidth $(200 \mathrm{~Hz})$. BigMag is capable of generating the fields of at least $40 \mathrm{mT}$ in an arbitrary direction at every point within its workspace. The field of each coil of BigMag can be controlled independently. Exploiting this property, highly non-homogeneous fields can be shaped. The gradients $\left(\|\nabla \mathbf{B}\|_{2}\right)$ of magnetic fields of up to $1 \mathrm{~T} / \mathrm{m}$ were measured inside the workspace. Such 
Table 2.2: Specification of BigMag Electromagnetic Coils

\begin{tabular}{lll}
\hline Specification & Iron-Cored Coil & Air-Cored Coil \\
\hline Inner Diameter $(\mathrm{mm})$ & 45 & 50 \\
\hline Outer Diameter $(\mathrm{mm})$ & $\begin{array}{l}\text { Stepped: } \\
\text { (Maximum) } 102\end{array}$ & 130 \\
\hline (Minimum) 56.3 & 50 \\
\hline Height $(\mathrm{mm})$ & 120 & - \\
\hline Core Length $(\mathrm{mm})$ & 180 & 1021 \\
\hline Resistance $(\Omega)$ & 1596 & 3.4 \\
\hline $\begin{array}{l}\text { Cut-off Frequency } \\
(\text { Approximately) }(\mathrm{Hz})\end{array}$ & 4.2 & 200 \\
\hline
\end{tabular}

gradients are insufficient for actuation of continuum manipulators, but can be used in steering of microrobotic agents using BigMag.

\subsection{Modelling of BigMag Coil Array}

In order to effectively steer a continuum manipulator, the magnetic field should be known at any location within the workspace of BigMag. This task is challenging, since the magnetic field generated by the mobile coils of the system is non-homogeneous. Moreover, the actual field $\left(\mathbf{B}_{\mathcal{G}}\left(\mathbf{p}_{n}\right)\right)$ varies depending on the positions of mobile coils. Therefore, it is essential to develop an accurate model of the field distribution for every state of the system. This section presents an inverse map technique, which relates the desired magnetic field $\left(\mathbf{B}_{\mathcal{G}}\left(\mathbf{p}_{n}\right)\right)$ to the set of BigMag configuration variables: coil currents $\left(\mathcal{I}=\left[\begin{array}{lll}I_{0} & \ldots & I_{5}\end{array}\right] \in \mathbb{R}^{6}\right)$ and coil fixture positions $\left(\theta_{1}, \theta_{2} \in \mathbb{S}\right)$.

The technique is derived in a few steps. First, the forward map of the array is introduced, describing $\mathbf{B}\left(\mathbf{p}_{n}\right)$ for a given configuration of BigMag. Due to rotating coils the forward map is highly non-linear, hence its analytical inversion is challenging. However, the over-actuation of BigMag allows reformulating the inverse map as an optimization problem minimizing power consumption of the array. We analyse the behaviour of this problem in simulation to propose an inverse map, which provides the desired solution taking into account the limits on coil currents and on fixture velocities.

\subsubsection{Forward Map of BigMag Coil Array}

In our model, a unit component of the array is a single coil, referred to with an index $(k \in[0,5])$. The coil has fixed dimensions and material composition. For every coil $(k)$ we construct a local reference frame $\left(\left\{\mathcal{C}_{k}\right\}\right)$ coincident with the long axis of the coil (Figure 2.3). The position of point $\left(\mathbf{p}_{n}\right)$ expressed in reference frame $\left(\left\{\mathcal{C}_{k}\right\}\right)$ is described by vector $\left(\mathbf{p}_{n}^{\mathcal{C}_{k}} \in \mathbb{R}^{3}\right)$. The coil is assumed to operate in 


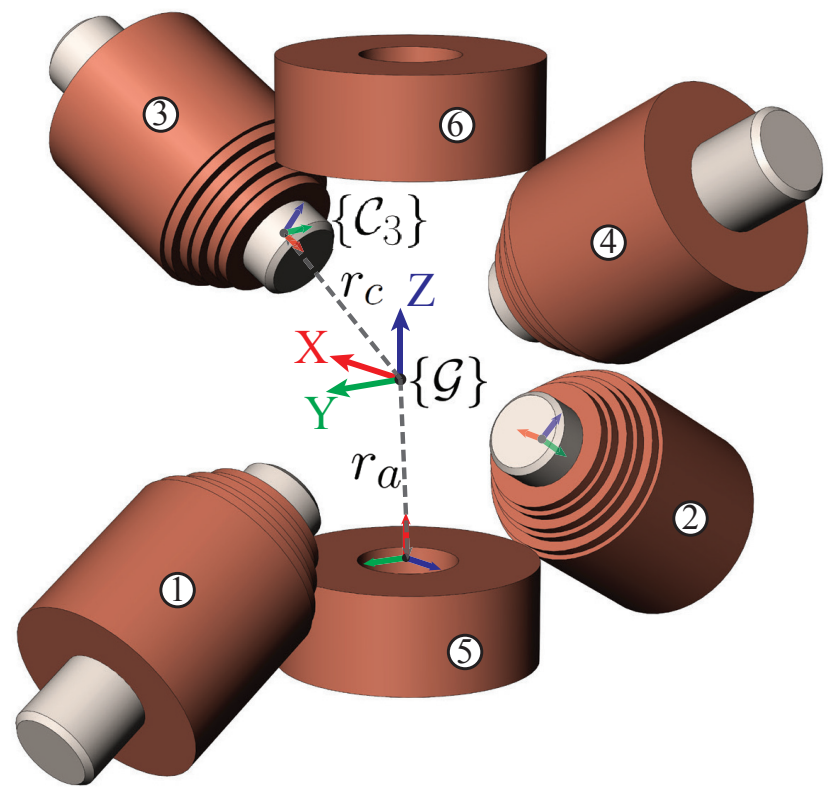

Figure 2.3: The overview of the proposed kinematic framework used in modelling of the BigMag coil array. The global reference frame $\{\mathcal{G}\}$ is located in the centre of the workspace. Local frames $\left\{\mathcal{C}_{k}\right\}, k \in \mathbb{N}, k \in[1,6]$, are placed on the long axis of the coils, on their faces oriented towards the workspace, such that their $X$ axes point towards the origin of frame $\{\mathcal{G}\}$. Variables $\left(r_{c}\right.$ and $r_{a}$ ) denote constant distances to BigMag coils. For $k \in[1,4], r_{k}=r_{c}=100 \mathrm{~mm}$, and for $k \in[5,6], r_{k}=r_{a}=110 \mathrm{~mm}$.

its linear region. Thus, the magnetic field $\left(\mathbf{B}_{\mathcal{C}_{k}} \in \mathbb{R}^{3}\right)$ at $\mathbf{p}_{n}^{\mathcal{C}_{k}}$ depends linearly on coil current $\left(I_{k} \in \mathbb{R}\right)$ and is given as follows:

$$
\mathbf{B}_{\mathcal{C}_{k}}\left(\mathbf{p}_{n}^{\mathcal{C}_{k}}\right)=I_{k} \boldsymbol{\beta}_{k}^{\mathcal{C}_{k}}\left(\mathbf{p}_{n}^{\mathcal{C}_{k}}\right),
$$

where $\boldsymbol{\beta}_{k}^{\mathcal{C}_{k}}\left(\mathbf{p}_{n}^{\mathcal{C}_{k}}\right) \in \mathbb{R}^{3}$ is the unit field map of coil $(k)$ at $\mathbf{p}_{n}^{\mathcal{C}_{k}}$ [179]. In this chapter we develop $\boldsymbol{\beta}_{k}^{\mathcal{C}_{k}}\left(\mathbf{p}_{n}^{\mathcal{C}_{k}}\right)$ using a measurement-based, axisymmetric representation similar to $[96]$.

The forward model of BigMag is constructed assuming the magnetic fields generated by each coil $(k)$ of BigMag follow the principle of superposition, providing the total field $\left(\mathbf{B}_{\mathcal{G}}\right)$. This field is expressed in the global reference frame $(\{\mathcal{G}\})$ located in the centre of BigMag workspace (Figure 2.2). The field map $\left(\boldsymbol{\beta}^{\mathcal{C}_{k}}\left(\mathbf{p}_{n}^{\mathcal{C}_{k}}\right)\right)$ is used to describe the effect of each coil on the total field. Since for each coil the map is expressed in local reference frame $\left(\left\{\mathcal{C}_{k}\right\}\right)$, we develop a coordinate transformation to express $\boldsymbol{\beta}^{\mathcal{C}_{k}}\left(\mathbf{p}_{n}^{\mathcal{C}_{k}}\right)$ in global reference frame.

Let us start by defining variables describing the kinematics of BigMag. The rotation matrix $\left({ }_{\mathcal{C}_{k}}^{\mathcal{G}} \mathbf{R} \in \mathrm{SO}(3)\right)$ describes the orientation of frame $\left(\left\{\mathcal{C}_{k}\right\}\right)$ for coil $(k)$ as a function of the position of coil fixture $\left(\theta_{f} \in \mathbb{S}\right)$, where $f \in\{1,2\}$. The displacement vector $\left(\mathbf{r}_{\mathcal{G}}^{\mathcal{C}_{k}} \in \mathbb{R}^{3}\right)$ describes the distance between origins of frames $\left(\{\mathcal{G}\}\right.$ and $\left.\left\{\mathcal{C}_{k}\right\}\right)$.

The position of point $\left(\mathbf{p}_{n}\right)$ in frame $(\{\mathcal{G}\})$ is described by $\mathbf{p}_{n}^{\mathcal{G}} \in \mathbb{R}^{3}$. Noting that 
$\mathbf{p}_{n}^{\mathcal{C}_{k}}=\mathbf{R}_{\mathcal{G}}^{\mathcal{C}_{k}} \mathbf{p}_{n}^{\mathcal{G}}+\mathbf{r}_{\mathcal{G}}^{\mathcal{C}_{k}}$, the unit field map $\left(\boldsymbol{\beta}_{k}^{\mathcal{G}}\left(\mathbf{p}_{n}^{\mathcal{G}}, \theta_{f}\right)\right)$ of each coil $(k)$, is represented as follows:

$$
\begin{array}{rl}
\boldsymbol{\beta}_{k}^{\mathcal{G}}\left(\mathbf{p}_{n}^{\mathcal{G}}, \theta_{f}\right) & ={ }_{\mathcal{C}_{k}}^{\mathcal{G}} \mathbf{R}\left(\theta_{f}\right) \boldsymbol{\beta}_{k}^{\mathcal{C}_{k}}\left(\mathbf{p}_{n}^{\mathcal{C}_{k}}\right) \\
\mathcal{G}_{k} & \mathbf{R}\left(\theta_{f}\right)={ }_{\mathcal{G}^{\prime}}^{\mathcal{G}} \mathbf{R}\left(\theta_{f}\right){ }_{\mathcal{C}_{k}}^{\mathcal{G}^{\prime}} \mathbf{R}^{0},
\end{array}
$$

where $\underset{\mathcal{G}^{\prime}}{\mathcal{G}} \mathbf{R}\left(\theta_{f}\right) \in \mathrm{SO}(3)$ is the rotation matrix around $Z$-axis of frame $(\{\mathcal{G}\})$ (Figure $2.2)$ by an angle $\left(\theta_{f}\right)$ and ${ }_{\mathcal{C}_{k}}^{\mathcal{G}} \mathbf{R}^{0} \in \mathrm{SO}(3)$ is the arbitrary reference configuration of coil $(k)$.

The design of BigMag allows for direct control over the currents $(\mathcal{I})$ in each coil and position of coil fixtures $\left(\boldsymbol{\theta}=\left[\begin{array}{ll}\theta_{1} & \theta_{2}\end{array}\right]^{T}\right)$. These variables are arranged into the input vector $\left(\boldsymbol{\eta}=\left[\begin{array}{ll}\mathcal{I}^{T} & \boldsymbol{\theta}^{T}\end{array}\right]^{T} \in \mathbb{R}^{8}\right)$. The total magnetic field as a function of input $(\boldsymbol{\eta})$ can be calculated using the forward map $\left(\gamma\left(\mathbf{p}_{n}^{\mathcal{G}}, \boldsymbol{\eta}\right)\right)$

$$
\begin{aligned}
\mathbf{B}_{\mathcal{G}} & =\gamma\left(\mathbf{p}_{n}^{\mathcal{G}}, \boldsymbol{\eta}\right)=\boldsymbol{\beta}\left(\mathbf{p}_{n}^{\mathcal{G}}, \boldsymbol{\theta}\right) \mathcal{I} \\
\boldsymbol{\beta}\left(\mathbf{p}_{n}^{\mathcal{G}}, \boldsymbol{\theta}\right) & =\left[\begin{array}{llll}
\boldsymbol{\beta}_{1}^{\mathcal{G}}\left(\mathbf{p}_{n}^{\mathcal{G}}, \theta_{1}\right) & \ldots & \boldsymbol{\beta}_{3}^{\mathcal{G}}\left(\mathbf{p}_{n}^{\mathcal{G}}, \theta_{2}\right) & \ldots
\end{array}\right] .
\end{aligned}
$$

The actuation of a magnetic catheter requires solving a converse problem, which takes the form of an inverse field map $\left(\gamma^{-1}\left(\mathbf{B}_{\mathcal{G}}, \mathbf{p}_{n}^{\mathcal{G}}\right)=\boldsymbol{\eta}\right)$, allowing for generation of arbitrarily prescribed magnetic fields inside the workspace of BigMag. These fields are related to torques deflecting the catheter by (1.1).

\subsubsection{Inverse Map of BigMag Coil Array}

The most complete way of finding $\gamma^{-1}\left(\mathbf{B}_{\mathcal{G}}, \mathbf{p}_{n}^{\mathcal{G}}\right)$ would involve analytical inversion of (2.4), this task is challenging due to the non-linearity of the forward map. Moreover, since the system is over-actuated, multiple solutions to the inverse map arise. Alternatively, the computation of the inverse map can be formulated as the following optimization problem:

$$
\begin{array}{ll}
\min _{\mathcal{I}} & \mathcal{I}^{T} \mathcal{I}, \\
\text { s.t. } & \mathbf{B}_{\mathcal{G}}-\boldsymbol{\beta}\left(\mathbf{p}_{n}^{\mathcal{G}}, \boldsymbol{\theta}\right) \mathcal{I}=0 .
\end{array}
$$

minimizing the power consumption of the array for given $\mathbf{B}_{\mathcal{G}}$. We note, that for arbitrarily selected coil fixture positions $(\boldsymbol{\theta})$ the constraint in (2.7) can be solved using the damped pseudo-inverse as follows:

$$
\begin{array}{r}
\mathcal{I}=\boldsymbol{\beta}^{\dagger} \mathbf{B}_{\mathcal{G}}, \\
\boldsymbol{\beta}^{\dagger}=\boldsymbol{\beta}^{T}\left(\boldsymbol{\beta} \boldsymbol{\beta}^{T}+\kappa^{2} \mathbf{I}_{6}\right)^{-1} \in \mathbb{R}^{6 \times 3} .
\end{array}
$$

The damping coefficient $\left(\kappa \in R^{+}, \kappa \ll 1\right.$.) is used to improve the behaviour of the solution $(\boldsymbol{I})$, if $\boldsymbol{\beta}$ becomes ill-conditioned [180].

The optimization problem given by (2.6) and (2.7) can be reduced by means of eliminating the constraint (2.7) with the help of the solution (2.8) and (2.9). 
Consequently, an equivalent form of (2.6) is obtained as follows:

$$
\min _{\mathcal{I}}\left(\mathcal{I}^{T} \mathcal{I}\right) \equiv \min _{\boldsymbol{\theta}}\left(\mathbf{B}_{\mathcal{G}}^{T} \boldsymbol{\beta}^{\dagger}\left(\mathbf{p}_{n}^{\mathcal{G}}, \boldsymbol{\theta}\right)^{T} \boldsymbol{\beta}^{\dagger}\left(\mathbf{p}_{n}^{\mathcal{G}}, \boldsymbol{\theta}\right) \mathbf{B}_{\mathcal{G}}\right)
$$

which involves evaluating the fixture positions $(\boldsymbol{\theta})$ for a given reference field $\left(\mathbf{B}_{\mathcal{G}}\right)$ and tip position $\left(\mathbf{p}_{n}^{\mathcal{G}}\right)$. Such a constraint elimination approach is valid due to the fact that the underdetermined system (2.7) has infinitely many solutions.

Nevertheless, solving (2.10) in real-time for catheter steering is challenging due to computational constraints. Thus, we propose a computationally fast approximate solution to (2.10). This solution is informed by a set of simulations (Figure 2.4 ), in which the cost function of the equivalent optimization problem (2.10) is computed for $\mathbf{B}_{\mathcal{G}}$ at 485 points $\left(\mathbf{p}_{n}^{\mathcal{G}}\right)$ distributed evenly across the workspace $\left(\boldsymbol{\Omega}_{w}\right)$. As follows from Figure 2.4, the center of the workspace $\left(\mathbf{p}_{n}^{\mathcal{G}}=\mathbf{0}_{3 \times 1}\right)$ the current minimizing coil fixture positions $\left(\boldsymbol{\theta}^{*}=\left[\begin{array}{ll}\theta_{1}^{*} & \theta_{2}^{*}\end{array}\right]^{T} \in \mathbb{S}^{2}\right)$ are given by

$$
\theta_{1}^{*}=\theta_{2}^{*}=\left(\operatorname{atan} 2\left(B_{y}^{\mathcal{G}}, B_{x}^{\mathcal{G}}\right)+n \pi\right)
$$

where $B_{x}^{\mathcal{G}}$ and $B_{y}^{\mathcal{G}}$ are $\mathrm{X}$, and $\mathrm{Y}$ components of $\mathbf{B}_{\mathcal{G}}$ respectively. Further, we notice that the optimal solutions for fields generated at other locations $\mathbf{p}_{n}^{\mathcal{G}}$ lie sufficiently close to (2.11) (standard deviation $0.237 \mathrm{rad}$ ), such that (2.11) can be considered an approximate solution of (2.10).

The approximate solution given by (2.11) has certain drawbacks for catheter steering. Since the mechanical bandwidth of the catheter is high, the prescribed (controlled) field $\left(\mathbf{B}_{\mathcal{G}}\right)$ should be generated quickly for a stable closed-loop system. However, steering the coil fixtures using (2.11) limits a fast response for $\mathbf{B}_{\mathcal{G}}$ due to the constraint on the velocity of the fixtures. Further, if the difference between the present fixture angle and (2.11) is high, the actual field at the intermediate angles can vary significantly. This issue can be solved by using the actual angles instead of (2.11) and recomputing (2.8) in the intermediate fixture positions. At the same time, (2.11) is provided as a reference setpoint for the servo system driving the fixtures.

A further improvement to the issues with (2.11) can be obtained by introducing the following offsets:

$$
\boldsymbol{\theta}^{*}=\left(\operatorname{atan} 2\left(B_{y}^{\mathcal{G}}, B_{x}^{\mathcal{G}}\right)+n \pi\right)\left[\begin{array}{ll}
1 & 1
\end{array}\right]^{T} \pm\left[\begin{array}{ll}
\theta_{1}^{o} & -\theta_{2}^{o}
\end{array}\right]^{T}
$$

to the approximate solution $\left(\boldsymbol{\theta}^{*}\right)$. Here, the angle $\left(\theta_{\star}^{o}\right)$, for $\star=\{1,2\}$ satisfies $\left(\theta_{\star}^{o} \in[\pi / 8, \pi / 4]\right)$. In Figure 2.4 it is shown that $(2.12)$ provides a better field response compared to $(2.11)$.

\subsubsection{Field Map Validation}

The inverse map is validated by prescribing a set field state trajectory (Figure 2.5) in 3 locations inside the workspace: at its centre $\left(\mathbf{p}_{n}^{\mathcal{G}}=\mathbf{0}_{3 \times 1} \mathrm{~mm}\right)$ and at two remote locations $\left(\mathbf{p}_{n}^{\mathcal{G}}=\left[\begin{array}{lll}30 & 30 & 0\end{array}\right]^{T} \mathrm{~mm}\right)$ and $\left(\mathbf{p}_{n}^{\mathcal{G}}=\left[\begin{array}{lll}28.9 & 28.9 & 28.9\end{array}\right]^{T} \mathrm{~mm}\right)$. 


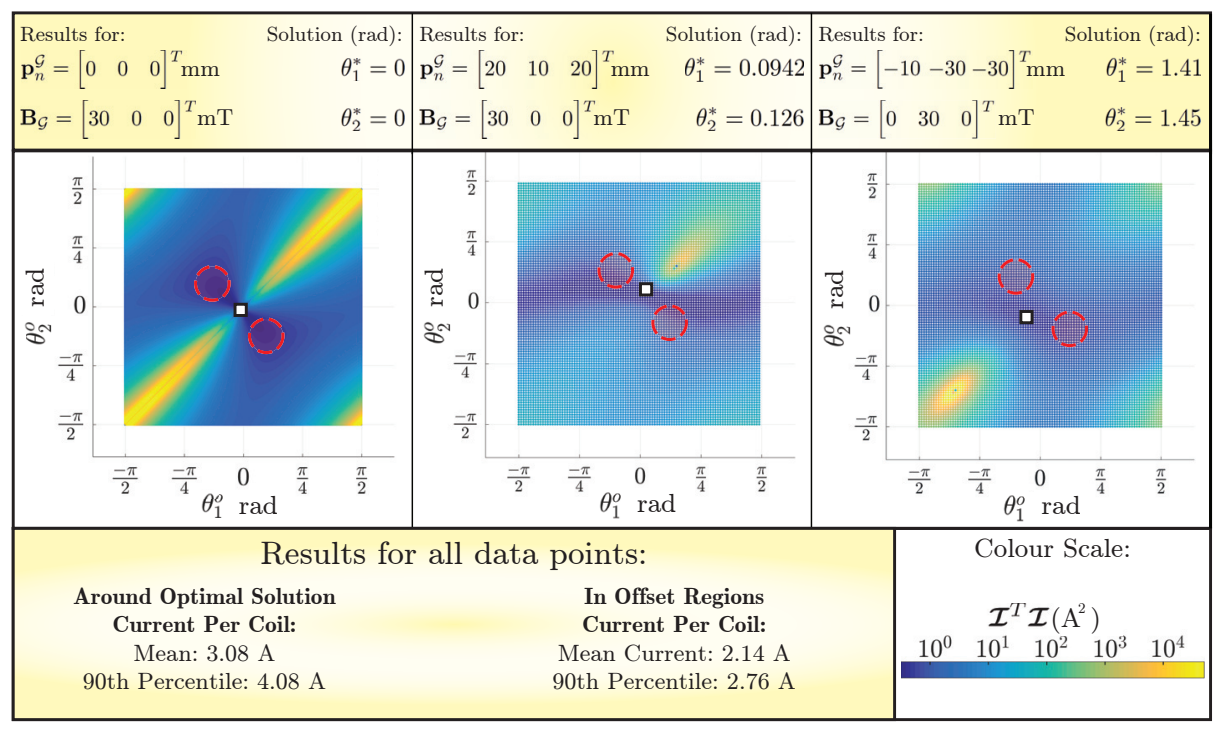

Figure 2.4: The effect of coil fixture positions on the BigMag coil currents are studied for a total of 485 evenly distributed points $\left(\mathbf{p}_{n}^{\mathcal{G}}\right)$ within the workspace of BigMag, for different values of magnetic field $\left(\mathbf{B}_{\mathcal{G}}\right)$. The positions of coil fixtures have no significant impact on fields generated in vertical direction. For other investigated $\mathbf{B}_{\mathcal{G}}$; the underdetermined system (2.7) is solved for coil currents $(\mathcal{I})$ for different fixture positions. These positions are located at an offset $\left(\theta_{1}^{o}, \theta_{2}^{o} \in[-\pi / 2, \pi / 2]\right)$ from the solution provided by (2.11). Representative results are shown above. For all $\mathbf{p}_{n}^{\mathcal{G}}$, the optimal coil fixture positions $\left(\boldsymbol{\theta}^{*}=\left[\begin{array}{ll}\theta_{1}^{*} & \theta_{2}^{*}\end{array}\right]^{T}\right)$ (white square) are found close (standard deviation $0.237 \mathrm{rad}$ ) to the solution proposed in (2.11). However, during the catheter steering, the actual fixture angles are used to compute $\mathcal{I}$. Those angles are likely to differ from optimal $\boldsymbol{\theta}^{*}$, provided as reference setpoint. Thus, we propose the use of solution (2.12), within the offset regions $\left(\theta_{1}^{o}, \theta_{2}^{o} \in[\pi / 8, \pi / 4]\right.$ ) (red circles), yielding on average a much better current per coil performance.

This state trajectory is executed using the inverse map field technique presented in Sections 2.3.1-2.3.2. Senis 3MH3A-500MT (Senis AG, Baar, Switzerland) Teslameter is used to measure the actual field in a desired location. In total 1500 samples are taken along each field state trajectory. The sets of desired and actual field samples are compared showing an average error of $2.20 \%$. The mean angle between the two field vectors is $2.82 \pm 1.15^{\circ}$, showing a significant improvement over preliminary method, presented in details in [179].

\subsubsection{Steering of Magnetic Catheters - Preliminary Test}

The final experiment is performed to evaluate the possibility of steering magnetic catheters inside a confined space using BigMag. A test bed is designed to mimic branching blood vessels. Several prototypes of magnetic catheters with different composition (see Table 2.3), are inserted into it using BigMag advancer. All prototypes bear a single, ring-shaped magnet mounted at their tip. Those catheters are examples of simple continuum manipulators. During the experiments the test bed is oriented vertically on the centre plane of the system. All six coils are aligned 


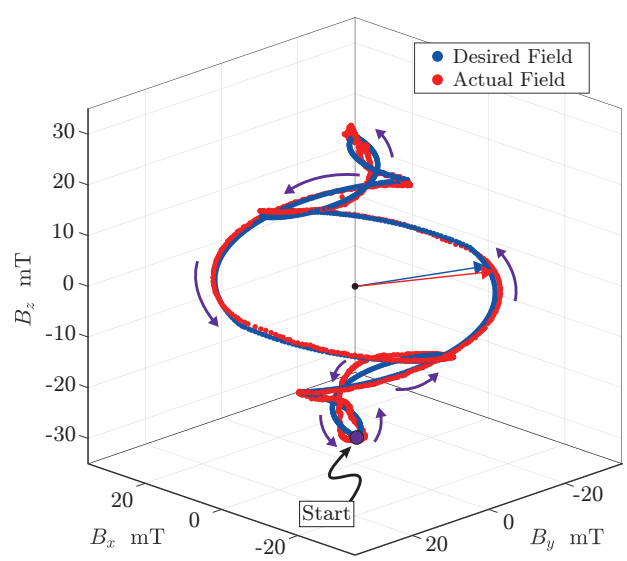

Figure 2.5: The inverse magnetic field map is validated by using it to generate desired magnetic field trajectories in 3 locations within the workspace of BigMag. The figure shows the values of the desired field vector (blue arrow) and the measured one (red arrow) for 1500 samples along the state trajectory generated at a point $\left(\mathbf{p}_{n}^{\mathcal{G}}=\left[\begin{array}{lll}28.9 & 28.9 & 28.9\end{array}\right]^{T} \mathrm{~mm}\right)$. The total percentage field magnitude error along all points from each state trajectory is $2.20 \%$ and the mean angle between field vectors is $2.82^{\circ}$.

with that plane. Figure 2.6 presents the methods and results.

\subsection{Conclusions and Future Work}

In this chapter we have presented BigMag, a novel platform using an array of mobile coils for 3D magnetic actuation of continuum manipulators. Furthermore, we have proposed and validated a mathematical model of its magnetic field, and proposed an inverse map technique for generation of prescribed magnetic fields. Finally, we have also demonstrated the application of BigMag in preliminary experiments involving manual steering of magnetic catheters.

BigMag fulfils all of the requirements established during its design process. It provides functional workspace sufficient for trials on exvivo specimen and on models of the human heart. The system generates magnetic fields of at least 40 $\mathrm{mT}$ in any direction within its workspace, and provides gradients of up to $1 \mathrm{~T} / \mathrm{m}$. The validation of the inverse field map shows its capability to generate magnetic fields with an average error magnitude of $2.20 \%$ and average error direction of $2.82^{\circ}$.

In this chapter we present only the experiments using manual steering of simple magnetic catheters. Hence, our next steps will involve demonstrating the full potential of the system. In Chapter 4, we will apply BigMag in automated, closedloop steering of continuum manipulators. Furthermore, in Chapter 5 we will use BigMag to enable the operation of an advanced tentacle catheter for underactuated grasping. 


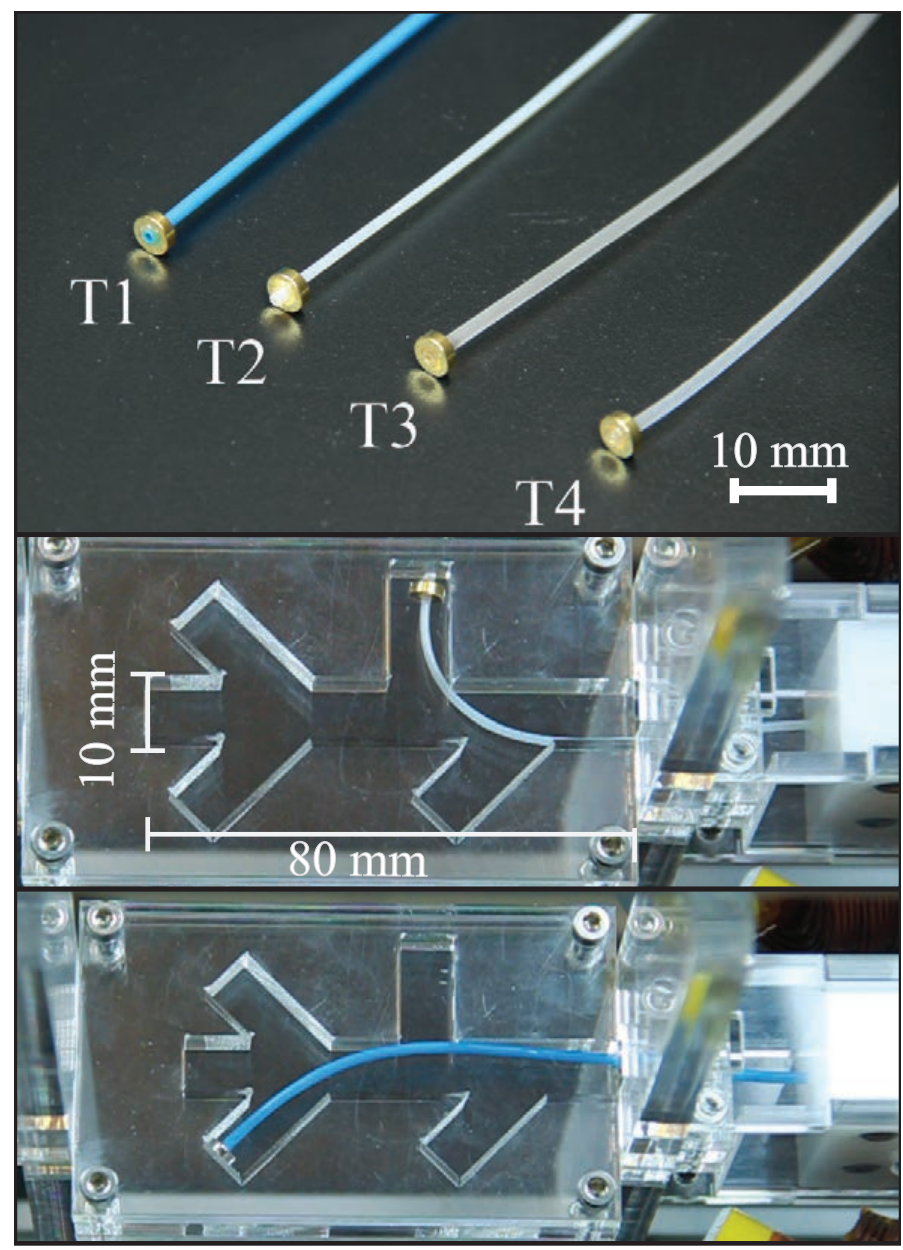

Figure 2.6: Top: Magnetic catheters described in Table 2.3. Centre, bottom: The test bed is a $10 \mathrm{~mm}$ wide horizontal channel with four branches, angled at $45^{\circ}$ and $90^{\circ}$. Currents in all coils and the position of the catheter inserter are all controlled by the operator using a keyboard to steer the catheters. All four devices are able to reach every prescribed destination during the experiments.

Table 2.3: Specification of the prototypes of magnetic catheters.

\begin{tabular}{lllll}
\hline Name & Material & $\begin{array}{l}\text { Inner } \\
\text { diameter } \\
(\mathbf{m m})\end{array}$ & $\begin{array}{l}\text { Outer } \\
\text { diameter } \\
(\mathbf{m m})\end{array}$ & $\begin{array}{l}\text { Young's } \\
\text { Modulus } \\
\mathbf{( M P a})\end{array}$ \\
\hline $\mathrm{T} 1$ & Pebax 6333 & 1.2 & 2.0 & 285 \\
\hline $\mathrm{T} 2$ & Unidentified & 1.1 & 1.5 & 100 \\
\hline $\mathrm{T} 3$ & PVC/DEHP & 1.2 & 2.2 & 50 \\
\hline $\mathrm{T} 4$ & $\begin{array}{l}\text { Polyurethane } \\
\text { 60A }\end{array}$ & 1.0 & 1.8 & 2.15 \\
\hline
\end{tabular}




\title{
Chapter 3
}

\section{The ARMM System: An Optimally-Designed Mobile Electromagnetic Coil for Non-linear Actuation of Flexible Surgical Instruments}

\begin{abstract}
Automation of flexible surgical instruments requires the development of robotic technologies capable of small-scale power transmission. Magnetic actuation has successfully been used for that purpose. Nevertheless, current systems for magnetic actuation suffer from small workspaces or poor bandwidth of magnetic field control. In this study, we design, develop, and test a novel magnetic actuation system called Advanced Robotics for Magnetic Manipulation (ARMM). The ARMM system employs a 6DoF mobile electromagnetic coil capable of generating prescribed magnetic fields and gradients. The mobile coil approach allows for easy scaling of the actuation workspace, which depends on the range of robotic arm, and in our case spans up to $1.3 \mathrm{~m}$. Due to limited end-effector payload of robotic arm used in the ARMM system, the mobile coil has been designed using an optimization routine. For given mass and heat dissipation constraints, this routine provides the coil geometry, which maximizes the average magnetic field generated in the target region. Since the Vacoflux core of the fabricated coil saturates within operational conditions, we propose an actuation strategy employing an online-updated iterative map technique. Using this map, the ARMM system allows for precise generation of prescribed magnetic fields and gradients at the point of interest, while taking into account the effects of the non-linearities due to core saturation. The strategy is validated experimentally, showing the average error of $2.34 \%$ for magnetic field and $7.20 \%$ for magnetic field gradient.
\end{abstract}

Adapted from:

J. Sikorski, C.M. Heunis, F. Franco, S. Misra. The ARMM system: An optimized mobile electromagnetic coil for non-linear actuation of flexible surgical instruments, IEEE Transactions on Magnetics vol. 55, no. 9, pp. 1-9, 2019 


\subsection{Introduction}

Steering and positioning of flexible surgical instruments, such as medical catheters in blood vessels during endovascular interventions is a challenging task, which may involve high-risk complications [181]. Although accessing most target areas within the human cardiovascular system is usually technically feasible, safe manual navigation of catheters inside these areas is highly dependent on the abilities of clinicians involved, requiring them to undergo expensive and time-consuming training [182]. Automating the guidance of catheters using the principles of surgical robotics can in the future mitigate those problems, vastly expanding the range of minimally-invasive procedures, which can be safely realised using such catheters.

Nevertheless, integrating surgical robotic technologies in endovascular catheters still requires the development of new actuation techniques, capable of transmitting power at small scales [65]. A range of promising catheter designs employs magnetic actuation for that purpose [134]. These catheters are fitted with ferromagnetic elements which, if subjected to an external magnetic field, experience magnetic wrenches due to Lorentz force. These wrenches comprise of both torque and force. The magnetic torque is dependent directly on the external magnetic field, whereas the magnetic force is determined by the spatial gradient of the field in the location of the magnetic element. These wrenches can be used for non-contact steering of a catheter inside the body of a patient, provided a system capable of controlling the field and its gradient in adequate volume [116,179].

Two principal classes of systems for magnetic actuation are presented in the literature. The first class involves those systems, which employ multiple electromagnetic coils organized into an array around a target volume $[106,116,179]$. These systems offer strong magnetic fields, although they exhibit poor workspace scalability, whereby increasing the target volume negatively affects the maximum control bandwidth of the system. Alternatively, several studies propose using mobile permanent magnets $[110,139,142,183,184]$. Those systems are capable of operating in larger workspaces. Nevertheless, as the strength of the field provided by permanent magnets cannot be changed, they require complicated actuator design and advanced motion planning algorithms for successful operation. Furthermore, the presence of non-decaying sources of magnetic field poses danger to personnel in the vicinity of the system, requiring safety protocol comparable to that of magnetic resonance systems.

The first notion that the advantages of both classes of magnetic actuation systems can be combined by using mobile electromagnetic coils was proposed by Veron et al., suggesting that mobile serial-link robotic actuators may prove to offer clinically-relevant workspaces, while decreasing labour costs, and improving ergonomics and workspace accessibility for the surgeons performing magneticallyguided procedures [185]. Several studies further explore the idea of using mobile coils for actuation of various medical instruments, however none of them presents a formalised design and implementation of such system [186-188].

Drawing inspiration from these ideas, in this chapter we propose a novel system for magnetic actuation. The ARMM (Advanced Robot for Magnetic Manipulation) system (Figure 3.1) employs a single, cored electromagnetic coil attached to a 6 DoF robotic arm. Due to coil mobility, it offers the benefit of much larger 


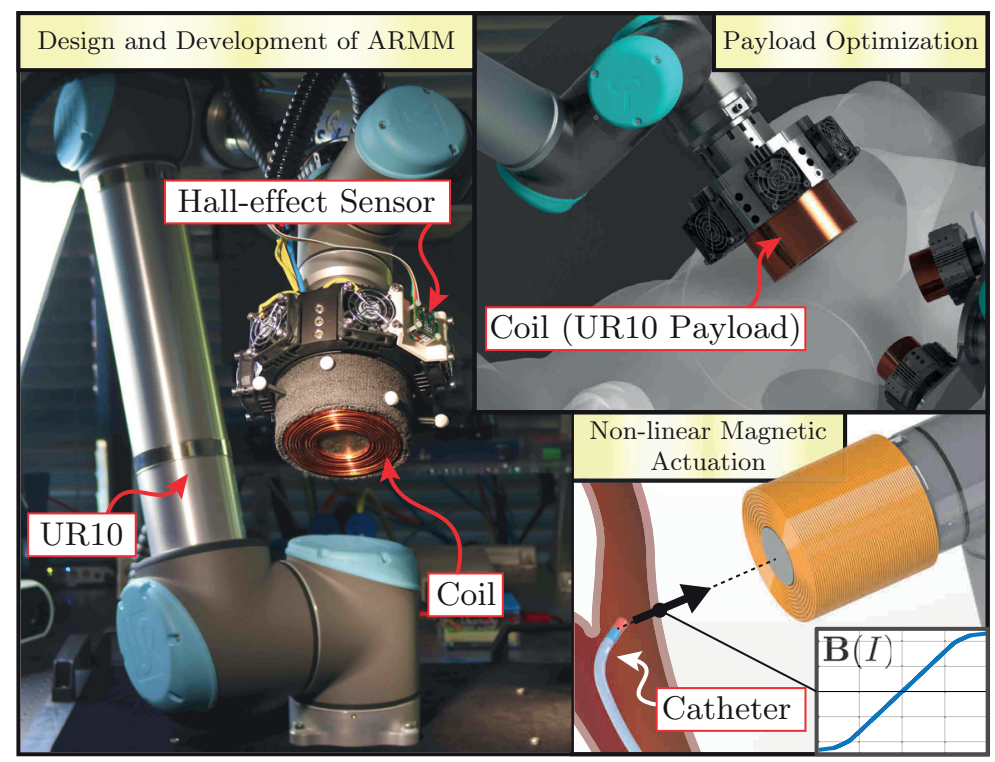

Figure 3.1: In this chapter, we present the design and development process of the ARMM (Advanced Robotics for Magnetic Manipulation) system. The ARMM system comprises 6DoF mobile electromagnet and is capable of providing magnetic actuation within a spherical workspace with a radius of $1300 \mathrm{~mm}$. Due to limited payload of the UR10 robotic arm (Universal Robots, Odense, Denmark) employed in the ARMM system, we propose an optimization strategy to design the electromagnet generating the largest magnetic field possible under a heat dissipation constraint. Furthermore, we propose a strategy to generate prescribed magnetic field $(\mathbf{B}(I))$ and its spatial gradient using the ARMM system. The strategy employs an online-updated iterative map, which uses a hall-effect sensor to account for the non-linearities of the field due to coil saturation at high currents.

workspace than the one provided by devices employing stationary electromagnets. Moreover, it alleviates challenges induced by systems employing permanent magnets as it provides greater range of controllable magnetic fields, including the capability to be switched off when necessary.

We begin the chpater by presenting a detailed design of the ARMM system in a context of a clinically-relevant catheter steering framework. We put particular emphasis on the design of the electromagnetic coil. As the limited payload of the robotic arm constrains the total size of the electromagnet, we propose an optimization routine which determines the composition of the coil resulting in maximum magnetic field generated for a given mass constraint. The routine takes into account the fact that the cross-section of the windings of the coil must result in coil resistance resulting in heat dissipation below a tolerable limit. Using optimization as a design tool has recently been proposed for linear, coreless electromagnets [189]. To authors best knowledge, this work is the first study aimed at designing a payload-optimal electromagnet with ferromagnetic core for magnetic actuation inside a clinically-relevant workspace.

The presence of a ferromagnetic core significantly increases the field generated by the mobile coil. Nevertheless, it complicates the modelling of the coil at higher currents, as the resulting field-to-current relation becomes non-linear once the core 
reaches its saturation limit [98]. The use of Hall-effect sensors for on-line correction of the coil model has been proposed to overcome this problem $[144,190]$. However, the approach presented therein neglects the fact that the superimposed magnetic fields of the core and the windings have different spatial distributions. This leads to a systematic correction error. In this work, we propose an alternative, exact approach for integrating Hall-effect sensors for on-line control of the electromagnetic coil. Thus, we can use the ARMM system for precise magnetic actuation past the saturation region.

Finally, we present an actuation strategy in which the ARMM system is used to generate co-directional, prescribed magnetic fields and forces with independently controlled magnitudes. We test the strategy in a series of experiments, whereby we control and measure the magnitudes of both the magnetic field and its spatial gradient. The latter quantity has been validated using a method previously applied in micro-electromechanical systems, in which the spatial gradient is measured indirectly, using a magnetically-compatible force sensor [191].

\subsection{Design of the ARMM system}

In this section, we present the composition of the ARMM system, which resulted from a cyclic process of material prototyping, testing, and iterative analysis. This section introduces the system integration of the ARMM system, including the catheter insertion device and the tracking capabilities of the system. Particular emphasis is put on the electromagnet design, which results from an optimization routine taking into account the limitations of the serial manipulator used in the system. The design parameters for the electromagnetic coil are determined by maximizing the average magnetic field in the operational region, while constraining the possible solutions, taking the tolerable coil temperature rise and payload limitations into account.

\subsubsection{System Composition and Magnetic Steering Strategy}

We begin the development process by analysing the principles of magnetic actuation in the context of catheters. This allows us to identify a set of requirements, which have to be fulfilled by a successful system for magnetic actuation. Magnetic actuation is used to exert a wrench $\left(\mathbf{W} \in \mathbb{R}^{3}\right)$ on a permanent dipole attached to a catheter. This dipole is located at a point $\left(\mathbf{p} \in \mathbb{R}^{3}\right)$ and is represented by a magnetic dipole moment $\left(\boldsymbol{\mu} \in \mathbb{R}^{3}\right)$. The magnetic wrench relates to the external magnetic field $\left(\mathbf{B}(\mathbf{p}) \in \mathbb{R}^{3}\right)$ and its spatial gradient as described by (1.1).

We identify three principal design requirements for a successful magnetic actuation system. First, it should be capable of deflecting a catheter in any direction by using magnetic wrench $(\mathbf{W})$. Furthermore, the magnitudes of torque and force components of $\mathbf{W}$ should be controllable, and as large as possible. Finally, a catheter used during an endovascular intervention needs to be navigated over large distances. Thus, a successful magnetic-actuation system should provide precise steering capabilities at every point along the required position trajectory, regardless of the pose of the catheter inside the body. 
The ARMM system, proposed in this chapter, is presented in Figure 3.2. It implements a $6 \mathrm{DoF}$ mobile coil approach, which allows generating magnetic fields and gradients in any direction in a scalable workspace, the size of which depends on the reach of the robotic arm used [185]. We use the UR10 (Universal Robots, Odense, Denmark), which has a spherical workspace with a radius of $1300 \mathrm{~mm}$ and can carry loads up to $10 \mathrm{~kg}$ with a $0.1 \mathrm{~mm}$ repeatability. Since it is a collaborative robot, its design ensures safety in clinical environments. The robot is located on the operative table alongside a UR5 (Universal Robots, Odense, Denmark) serial arm, used to position sensors or medical imaging devices, such as ultrasound transducers.

The steering strategy employed for the actuation of a catheter using the ARMM system assumes knowledge of the point $(\mathbf{p})$, at which the dipole $(\boldsymbol{\mu})$ attached to the catheter is located. We fit a local reference frame $(\{\mathcal{T}\})$ onto the front face of the coil, such that the coil symmetry axis $\left(\mathbf{Z}_{C} \in \mathbb{R}^{3}:\left\|\mathbf{Z}_{C}\right\|=1\right)$ is coincident with the $Z$ axis of $\{\mathcal{T}\}$. Subsequently, we assume that the UR10 robot is capable of positioning the coil, such that $\mathbf{p}$ is coincident with $\mathbf{Z}_{C}$ at all times (Figure 3.2). Thus, the magnetic field at $\mathbf{p}$ can be expressed as follows:

$$
\mathbf{B}(\mathbf{p}, I)=\mathbf{Z}_{C} B(z, I),
$$

where $z \in \mathbb{R}^{+}$is the distance of the dipole $(\boldsymbol{\mu})$ from the face of the coil along the axis $\left(\mathbf{Z}_{C}\right)$ and $B(z, I): \mathbb{R}^{+} \times \mathbb{R} \mapsto \mathbb{R}$ is the scalar value of the magnetic field along the axis $\left(\mathbf{Z}_{C}\right)$. If the rotational matrix $\left(\mathbf{R}_{\mathcal{T}} \in \mathrm{SO}(3)\right)$ describes the orientation of the coil frame $(\{\mathcal{T}\}),(1.1)$ can be reduced to

$$
\begin{aligned}
& {\left[\begin{array}{c}
\mathbf{F}_{\mu} \\
\boldsymbol{\tau}_{\mu}
\end{array}\right]=\left[\begin{array}{cc}
\mathbf{0}_{3 \times 1} & \mathbf{R}_{\mathcal{T}} \nabla \mathbf{B}_{0} \mathbf{R}_{\mathcal{T}}^{T} \boldsymbol{\mu} \\
S(\boldsymbol{\mu}) \mathbf{Z}_{C} & \mathbf{0}_{3 \times 1}
\end{array}\right] \underbrace{\left[\begin{array}{lll}
B(z, I) & \frac{\partial B}{\partial z}
\end{array}\right]^{T}}_{\mathbf{b}},} \\
& \nabla \mathbf{B}_{0}=\operatorname{diag}\left(\left[\begin{array}{lll}
-0.5 & -0.5 & 1
\end{array}\right]\right)
\end{aligned}
$$

This choice of actuation technique is motivated by previous studies involving steering of magnetic catheters. The magnetic torques, generated have been shown to have a higher impact on the deflection than magnetic forces, hence the effect of the latter is often neglected $[144,174,179]$. In the same time, our technique ensures presence of a force coincident with the torque bending plane, improving the stability of the catheter at larger deflections [118]. Furthermore, the UR10 robot can reposition itself with speeds of up to $1 \mathrm{~m} / \mathrm{s}$, which is sufficient to follow catheters, which move significantly slower [155].

In previous studies, the torque $\left(\tau_{\mu} \in \mathbb{R}^{3}\right)$ has been identified as the primary component of $\mathbf{W}$ used to deflect flexible magnetic catheters. As follows from (3.2), both the magnitude and the direction of this torque are proportional to the magnitude of the magnetic field $(B(z, I))$. Thus, designing an end-effector electromagnetic coil for the ARMM system should aim at maximizing the magnetic fields generated by it at a certain distance $(z)$ from its face. As the coil is positioned outside the human body, we assume that the catheter at any instance during the procedure is located between 5 and $20 \mathrm{~cm}$ away from the coil [192]. Thus, our aim is to design a coil which maximizes field generation within that region. 


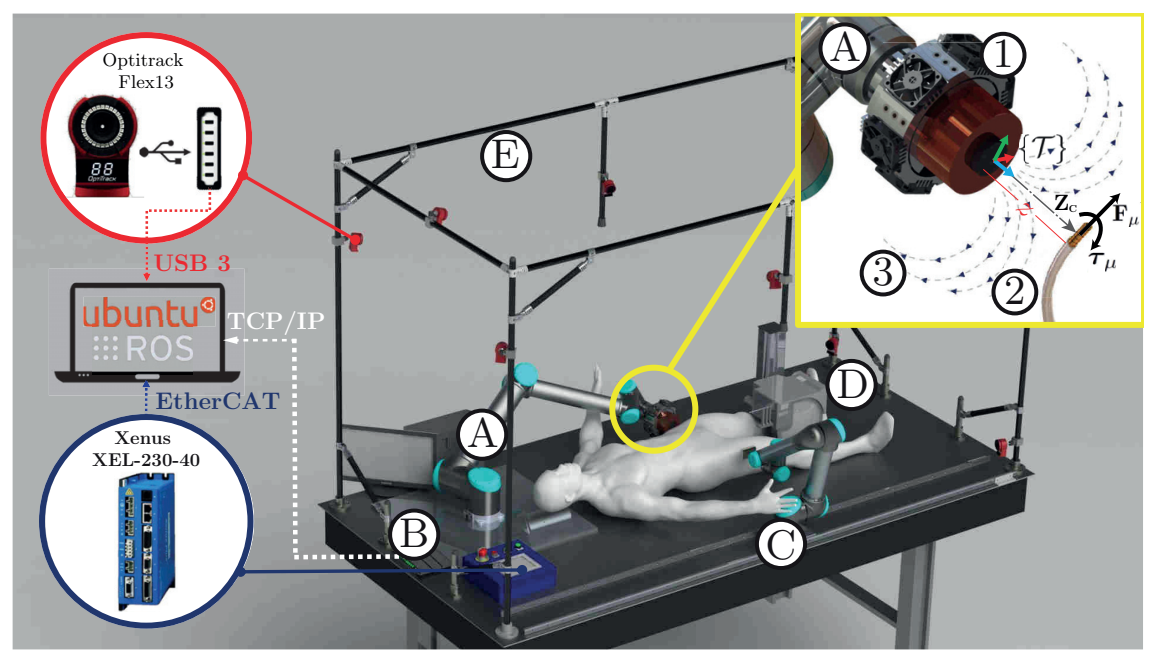

Figure 3.2: A The ARMM (Advanced Robotics for Magnetic Manipulation) system is a magnetic actuation system that is designed to guide flexible surgical instruments, such as an endovascular catheters, in a clinically-relevant workspace. The framework supporting the operation of the ARMM system includes (B) a digital control network, (C) an assistive robotic arm UR5 (Universal Robots, Odense, Denmark) - in this study used for sensor positioning, and (D) a catheter insertion device. The workspace is surrounded by a truss structure (E), containing eight Optitrack Flex 13 (NaturalPoint, Inc., Corvallis, USA) cameras used for registering and tracking objects inside the workspace. The magnetic actuation strategy employed in the ARMM system uses a mobile coil (1) to steer an endovascular catheter (2) by generating magnetic field (3). When exposed to this field, a catheter equipped with a magnetic dipole experiences magnetic force $\left(\mathbf{F}_{\mu} \in \mathbb{R}^{3}\right)$ and torque $\left(\boldsymbol{\tau}_{\mu} \in \mathbb{R}^{3}\right)$. UR10 is used to align coil axis of symmetry $\left(\mathbf{Z}_{C} \in \mathbb{R}^{3}:\left\|\mathbf{Z}_{C}\right\|=1\right)$ with the centre of the catheter tip. Such configuration allows to control the values of $\mathbf{F}_{\mu}$ and $\boldsymbol{\tau}_{\mu}$ by varying the current $(I \in \mathbb{R})$ in the coil and the distance $\left(z \in \mathbb{R}^{+}\right)$from the dipole to the face of the coil. (C)Christoff M. Heunis, 2019)

\subsubsection{Payload-Optimized Design of a Mobile Electromagnet}

In contrast to other systems, where stationary coils can be made as large as possible to generate fields within the desired specifications, the UR10 robotic arm used in the ARMM system can carry a maximum payload $\left(m_{m}=10 \mathrm{~kg}\right)$, which limits the maximum size of the coil. This problem can be overcome by driving the coil with larger maximum current $\left(I=I_{m}\right)$, although this can lead to excessive coil heat dissipation. Thus, we employ an optimization routine, allowing us to determine the optimal design of the coil, maximizing actuation capabilities of the coil under payload and heat dissipation constrains.

We limit our investigation to ferrous-core solenoid coils, due to their reliability and ease of manufacturing. The geometry of such a coil (Figure 3.3) can be described by a cylinder with length $(l \in \mathbb{R})$. The inner portion of the cylinder is occupied by the ferromagnetic core with average density $\left(\rho_{c} \in \mathbb{R}^{+}\right)$and radius $\left(r_{i} \in \mathbb{R}^{+}\right)$. The rest of the coil comprises of $\left(n \in \mathbb{R}^{+}\right)$turns of copper wire, with average density $\left(\rho_{w} \in \mathbb{R}^{+}\right)$, electrical resistivity $\left(\alpha \in \mathbb{R}^{+}\right)$and diameter $\left(w \in \mathbb{R}^{+}\right)$. The turns are organized in as many layers as possible, not exceeding the outer radius of the coil $\left(r_{o} \in \mathbb{R}^{+}\right)$. The maximum number of turns of the wire $(n)$, which 


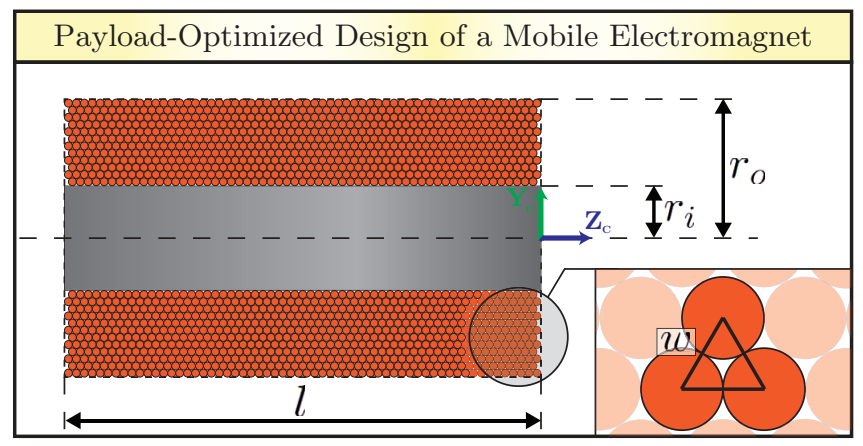

Figure 3.3: The model of coil geometry used in the optimization routine presented in this chapter. The coil comprises two individual parts: a cylindrical core and $n$ windings of copper wire with diameter $\left(w \in \mathbb{R}^{+}\right)$. The length $\left(l \in \mathbb{R}^{+}\right)$of both the core and the windings is the same. The core occupies inner portion of the coil, up to the distance $\left(r_{i} \in \mathbb{R}^{+}\right)$, whereas the windings are arranged around it into layers reaching up to the final radius of the core $\left(r_{o} \in \mathbb{R}^{+}\right)$. The proposed optimization routine finds the combination of parameters $\left(w, r_{i}, r_{o}, l\right)$ resulting in the highest magnetic field in the desired workspace while taking into account the temperature and payload constraints.

can be assembled into a coil with certain geometry depends on the manufacturerspecific coil filling factor. For the best case scenario (Figure 3.3) $n$ is given by

$$
n=\left\lfloor\frac{4 l(h-2 w) \sqrt{3}}{6 w^{2}}+\frac{l}{w}-\frac{(h-w) \sqrt{3}}{3 w}-\frac{1}{2}\right\rfloor,
$$

where $\left(h=r_{o}-r_{i}\right)$, and \lfloor\rfloor is the flooring operator.

The two variables used to constrain the proposed optimization are the mass $\left(m \in \mathbb{R}^{+}\right)$and thermal power $\left(P \in \mathbb{R}^{+}\right)$of the solenoid. They can be calculated as follows:

$$
\begin{aligned}
m & =\pi l\left(\rho_{w}\left(r_{o}^{2}-r_{i}^{2}\right)+\rho_{c} r_{i}^{2}\right) \\
P & =I_{m}^{2} \alpha \frac{4 n h}{w^{2}} .
\end{aligned}
$$

Relation (3.5) determines directly the set of possible coil geometry parameters $\left(r_{o}, r_{i}, l, w\right)$ constraining the mass of the coil below the payload limit. Thus, (3.5) is used as a constraint in the optimization scheme. On the other hand, determining the maximum acceptable thermal power of the coil is challenging, due to the complexity of models describing heat dissipation within the material. Thus, in order to convert (3.6) into a practical constraint, we decide to consider a worst case heating scenario. We assume that the coil is a closed thermal system, i.e. no heat is exchanged with the environment. This allows to relate the thermal power to a predicted temperature rate $(\dot{T} \in \mathbb{R})$ of the coil running continuously at $I_{m}$ as follows:

$$
\dot{T}=P C_{a}
$$


where $\left(C_{a} \in \mathbb{R}^{+}\right)$is the heat capacity of the coil approximated by the following relation:

$$
C_{a}=\pi l\left(c_{w} \rho_{w}\left(r_{o}^{2}-r_{i}^{2}\right)+c_{c} \rho_{c} r_{i}^{2}\right),
$$

using the specific heats of wire $\left(c_{w} \in \mathbb{R}^{+}\right)$and of the core $\left(c_{c} \in \mathbb{R}^{+}\right)$respectively. We select the maximum temperature rate as a constraint for our optimization problem, as it can be easily related to total expected coil heating for a procedure of a given time. Since in clinical scenario the use of maximum current throughout the entire procedure is unlikely, the temperature rate calculated using (3.7) is likely to be overestimated. This, in turn, should further improve the thermal safety of the design.

Please note that we disregard the dependency of wire resistivity $(\beta)$ on coil temperature. It can be ignored as long as the total temperature increase of the coil is kept within $25-40{ }^{\circ} \mathrm{C}$ (resistivity increase of $10-15$ [\%]) [193]. It is valid to assume that a clinical system would operate within this temperature range, as higher temperatures can pose a threat to clinicians and patients and should be avoided [108].

Subsequently the optimal geometry of the coil can be calculated by solving the following maximization problem:

$$
\begin{array}{ll} 
& \max _{r_{o}, r_{i}, l, w \in \mathbb{R}^{+}} \beta(B(z, I)) \\
\text { s.t. } & m \leq m_{m}, \dot{T} \leq \dot{T}_{m}, r_{i}<r_{o},
\end{array}
$$

where the function $\left(\beta(B(z, I)): \mathbb{R}^{+} \times \mathbb{R} \mapsto \mathbb{R}^{+}\right)$provides information about the optimality of the particular field distribution $(B(z, I))$ and depends on the design requirements. In case of the coil designed for the ARMM system, we maximize the average field generated along the long axis $\left(\mathbf{Z}_{C}\right)$ of the coil, between 5 and $20 \mathrm{~cm}$ away from the face of the coil. Thus, the proposed $\beta(B(z, I))$ is as follows:

$$
\beta(B(z, I))=\frac{1}{0.15} \int_{0.05}^{0.2} B\left(z, I_{m}\right) d x
$$

Since $B(z, I)$ for a given coil geometry is challenging to establish analytically, it is usually approximated using simplified models [117] or finite-element simulations [179].

\subsubsection{Final Coil Design}

The optimization problem (3.9) is solved taking into account the constraints established in the process of designing the ARMM system. The maximum mass $\left(m_{m}\right)$ was set to $8 \mathrm{~kg}$ to leave enough payload for fasteners and sensors. The maximum tolerable initial temperature rate $\left(\dot{T}_{m}\right)$ is set arbitrarily at $0.1 \mathrm{~K} / \mathrm{s}$, since we assume the ARMM system will be used for tasks requiring no more than 3 minutes of maximum field generation at any given time. A parametric model of the coil is developed using COMSOL 5.2 (COMSOL, Burlington, USA) finite-element solver and linked with MATLAB 2017b (MathWorks, Natick, USA) routine using LiveLink utility. By solving the model, we obtain the field distribution for a 
Table 3.1: Specifications of the ARMM (Advanced Robotics for Magnetic Manipulation) system electromagnetic coil

\begin{tabular}{ll} 
Specification / characteristic & Value / description \\
\multicolumn{2}{c}{ Copper-wire electromagnetic coil } \\
(Wijdeven, Oirschot, & Netherlands $)$ \\
\hline Outer diameter $\left(2 r_{o}\right)(\mathrm{mm})$ & 105 \\
Inner diameter $\left(2 r_{i}\right)(\mathrm{mm})$ & 45 \\
Length $(l)(\mathrm{mm})$ & 110 \\
Wire diameter $(w)(\mathrm{mm})$ & 1.6 \\
Turns $(n)$ & 1282 \\
DC Resistance $(\Omega)$ & 2.6 \\
Specific heat $\left(\frac{\mathrm{J}}{\mathrm{kg} * K}\right)$ & 400 \\
Density $\left(\frac{\mathrm{kg}}{\mathrm{m}^{3}}\right)$ & 8500 \\
Resistivity $\left(\frac{\Omega}{\mathrm{m}}\right)$ & $1.7 * 10^{-8}$ \\
\multicolumn{1}{c}{ Vacoflux 50 core $($ Sekels, Ober-Mörlen, Germany) } \\
\hline Length $(\mathrm{mm})$ & 135 \\
Density $\left(\frac{\mathrm{kg}}{\mathrm{m}^{3}}\right)$ & 8120 \\
Treatment & Magnetic annealing; \\
Saturation magnetization $(\mathrm{T})$ & Corrosion protection \\
\hline
\end{tabular}

given set of parameters $\left(r_{o}, r_{i}, l, w\right)$. The average computation time for a solution corresponding to a particular geometry is $2.7 \mathrm{~s}$. Results of the optimization are presented in Figure 3.4, whereas the parameters of the coil manufactured using the geometry calculated by our optimization scheme are provided in Table 3.1.

The prototype of the coil is assembled and characterized by using a teslameter to measure the magnetic field $(B(z, I))$ at a distance $(z=100 \mathrm{~mm})$ generated at a full, operational range of currents $(I)$. Subsequently, the same procedure is performed without the Vacoflux core. The results (Figure 3.5) are compared to the outcome of the COMSOL model used during the design. We observe that the total field generated by the coil is larger than the model predictions. This is mostly due to the magnetization of the Vacoflux core, as the difference between the experimental data and the model for the magnetic field of the coil windings without the core is negligible. The field generated by the core exhibits non-linearities due to saturation in its operational region (in contrary to predictions from the model). In the remainder of this chapter, we propose a novel magnetic actuation strategy, which takes this phenomenon into account.

\subsection{Non-linear Magnetic Actuation Using The ARMM System}

In this section, we propose a non-linear, iterative technique, for the precise, realtime generation of prescribed magnetic fields and gradients using the ARMM sys- 

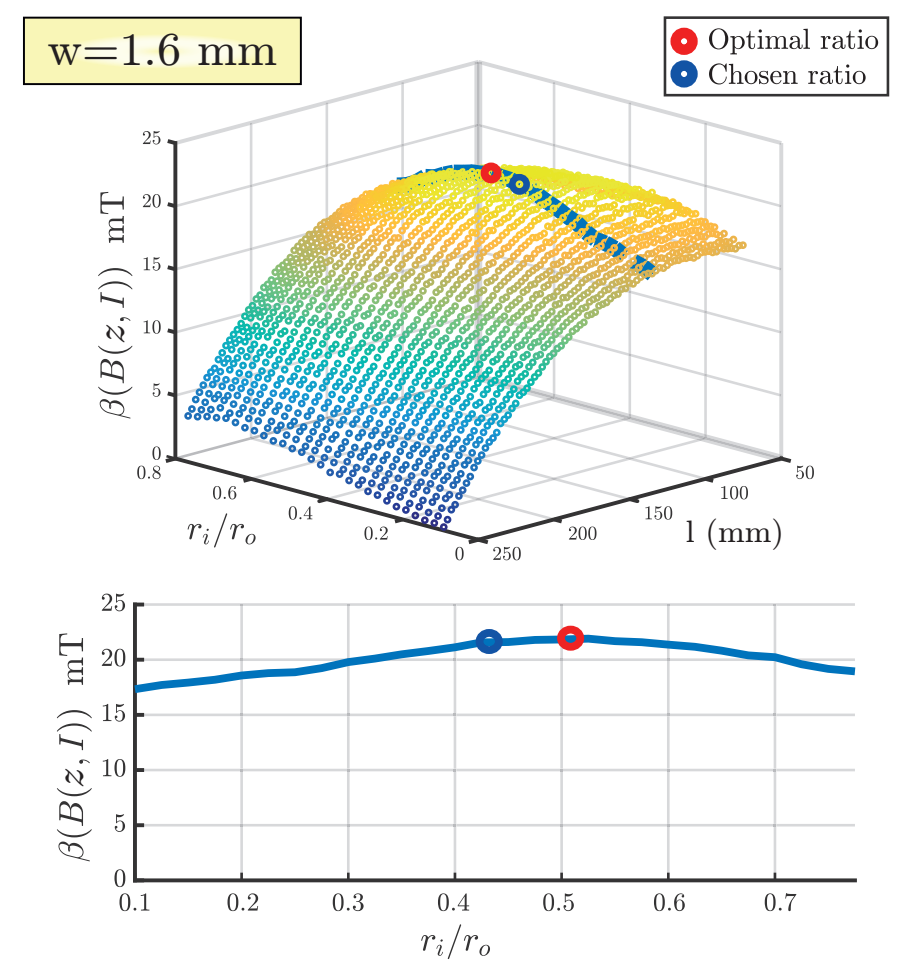

Figure 3.4: The optimization routine (3.9) is employed in the design of the electromagnetic coil for the ARMM (Advanced Robotics for Magnetic Manipulation) system. Since the full fourdimensional optimization space is impossible to visualise, we reduce the number of variables to highlight the main findings of the procedure. The top graph shows a selection of results for the final wire diameter $(w=1.6 \mathrm{~mm})$. The results are generated for a selection of coil lengths $(l)$ and ratios of the inner and outer radii of the coil $\left(r_{i} / r_{o}\right)$. The value of the radii are computed using (3.5) such that the mass of the coil is as large as possible for each ratio. The bottom graph shows the cross section of the results for the optimized length $(l=110 \mathrm{~mm})$ resulting in highest average value of the field $\beta(B(z, I))$. The optimal $r_{i} / r_{o}$ is 0.527 , resulting in the inner radius of $25.2 \mathrm{~mm}$. However, seeing the neighbourhood of the final solution, we notice that the dependence of the solution on the ratio $r_{i} / r_{o}$ is not significant. Thus, without losing much performance, we select a smaller ratio of 0.425 , which results in the inner diameter of $45 \mathrm{~mm}$, corresponding to the size of the coiling tool available to our manufacturer.

tem. This technique employs an online-updated inverse map of ARMM to compute the configuration of the system generating reference magnetic fields and gradients. Our approach can be used to generate mutually orthogonal magnetic forces and torques with independently controlled magnitudes.

The task of the iterative map is to find the appropriate configuration of the ARMM system $\left(\mathbf{q}_{m}=\left[\begin{array}{ll}z & I\end{array}\right]^{T}\right)$, corresponding to the reference magnetic actuation variables (b) as defined in (3.2). Since the coil used in the ARMM system exhibits saturation in its operational region, we model separately the magnetic field components generated by the windings and by the Vacoflux core. Hence, the total field $(B(z, I))$ can be modelled as a superposition of those two components 


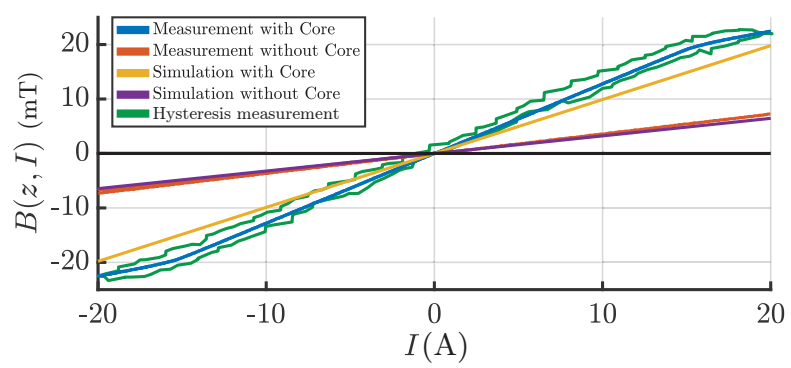

Figure 3.5: A Senis 3MH3A-500MT (Senis AG, Baar, Switzerland) teslameter is used to measure the value of the magnetic field $(B(z, I))$ generated by the coil of the ARMM (Advanced Robotics for Magnetic Manipulation) system at the location $(z=0.1 \mathrm{~m})$ in the entire operational range of current $(I)$. The results are compared to predictions from the FE model used in the design process. The measurements and simulations are performed for the coil with and without the Vacoflux core. The graph shows that the coil produces more magnetic field than predicted by the model. This is mostly due to the better magnetic properties of the core. Furthermore, an additional fast sweep (green) across the entire operational current range in $0.5 \mathrm{~s}$. This was performed to investigate the presence of hysteretic behaviour during sudden current variations, as motivated by our previous experience with similar electromagnets. Limited hysterietic behaviour is observed. However, since such sudden variations in current are unlikely to occur during catheter steering tasks, we neglect this behaviour. Nevertheless, the coil exhibits saturation in the operational range. As this phenomenon makes the actuation non-linear for any rate of current, it needs to be taken into account while using ARMM for precise magnetic actuation.

as follows:

$$
\begin{aligned}
B(z, I) & =I\left(B_{w}(z)+\eta(I) B_{c}(z)\right) \\
\frac{\partial B}{\partial z}(z, I) & =I\left(\frac{\partial B_{w}}{\partial z}+\eta(I) \frac{\partial B_{c}}{\partial z}\right),
\end{aligned}
$$

where $\eta(I): \mathbb{R} \mapsto(0 ; 1]$ is the saturation model of the core, and $B_{w}, B_{c} \in \mathbb{R}^{+}$are the unit field maps of the windings and the core at the distance $(z)$.

Furthermore, we can relate the rates of change of $\mathbf{b}$ and $\mathbf{q}_{m}$ through a Jacobian $\operatorname{matrix}\left(\mathbf{J} \in \mathbb{R}^{2 \times 2}\right)$

$$
\dot{\mathbf{b}}=\mathbf{J} \dot{\mathbf{q}}_{m}=\left[\begin{array}{cc}
\frac{\partial B}{\partial z} & \frac{\partial B}{\partial I} \\
\frac{\partial^{2} B}{\partial z^{2}} & \frac{\partial^{2} B}{\partial I \partial z}
\end{array}\right] \dot{\mathbf{q}}_{m}
$$

Using the magnetic field model $(3.12-3.13)$, we derive the entries of the Jacobian as follows:

$$
\begin{gathered}
\frac{\partial^{2} B}{\partial I \partial z}=\frac{\partial B_{w}}{\partial z}+\left(I \frac{d \eta}{d I}+\eta(I)\right) \frac{\partial B_{c}}{\partial z}, \\
\frac{\partial^{2} B}{\partial z^{2}}=I\left(\frac{\partial^{2} B_{w}}{\partial z^{2}}+\eta(I) \frac{\partial^{2} B_{c}}{\partial z^{2}}\right) \\
\frac{\partial B}{\partial I}=B_{w}(z)+\left(I \frac{d \eta}{d I}+\eta(I)\right) B_{c} .
\end{gathered}
$$


We use $(3.14$ - 3.17) in an iterative Jacobian inverse scheme presented in Figure 3.6. The unit field maps $\left(B_{c}, B_{w}\right)$ and their spatial derivatives are represented using rational functions of the form

$$
B_{\star}(z)=\frac{A_{\star}}{z^{3}}+\frac{B_{\star}}{z^{2}}+\frac{C_{\star}}{z}+D_{\star}, \star=\{c, w\},
$$

with coefficients $\left(A_{\star}, \ldots, D_{\star} \in \mathbb{R}\right)$ fitted to field measurements at a set of discrete locations along $z$.

We estimate $\eta$ using a MLX90393 3-axis Hall-effect sensor (Melexis NV, Ieper, Belgium) located at a point $\left(\mathbf{p}_{s}\right)$ (Figure 3.8). First, we use the sensor to create unit field maps $\left(\mathbf{B}_{w}\left(\mathbf{p}_{s}\right)\right)$ and $\left(\mathbf{B}_{c}\left(\mathbf{p}_{s}\right)\right)$ for windings and core at $\mathbf{p}_{s}$, analogously to (3.12). These field maps are subsequently used during the on-line estimation phase, alongside the real-time field measurement $\left(\mathbf{B}\left(\mathbf{p}_{s}\right)\right)$. In that phase, $\eta$ is calculated as follows:

$$
\begin{array}{r}
\eta=\frac{\left\|\mathbf{B}\left(\mathbf{p}_{s}\right)\right\|-I\left\|\mathbf{B}_{w}\left(\mathbf{p}_{s}\right)\right\|}{I\left\|\mathbf{B}_{c}\left(\mathbf{p}_{s}\right)\right\|} \text {, for } I \neq 0, \\
\eta=1, \text { for } I=0,
\end{array}
$$

Equation (3.20) holds, since for $I=0$ we can readily assume no saturation. Hence, we can use the relationship $\left(\left\|\mathbf{B}\left(\mathbf{p}_{s}\right)\right\|=I\left\|\mathbf{B}_{c}\left(\mathbf{p}_{s}\right)\right\|+I\left\|\mathbf{B}_{w}\left(\mathbf{p}_{s}\right)\right\|\right)$ to reduce (3.19). In practice, we extend the range of (3.20) to $|I|<0.1 \mathrm{~A}$, since for low currents, the signal-to-noise ratio and the resolution for both the current and field sensors are too low for accurate on-line estimation. At the same time, the derivative $\left(\frac{d \eta}{d I}\right)$ is approximated using a backwards finite-difference scheme.

The presented iterative map constitutes only the innermost cascade in closedloop control of magnetic instruments. Realisation of actual position control is beyond the scope of this study, since it requires substantial work related to modelling and tracking of the catheter. In practice, our iterative map could be implemented in a way similar to our previous approach [194]. ARMM could replace the actuator array used therein, which is also driven by an inverse-map approach. Alternatively, the Jacobian derived in (3.14), could be extended to account for direction of the field/gradient and coupled with model of the catheter dynamics, in a fashion similar to [116].

\subsection{Implementation and Experimental Results}

The iterative map technique presented in Section 3.3 is implemented in $\mathrm{C}++14$ on a personal computer running Linux Ubuntu 14.04. Ethernet communication is used to interface with the UR10 robot using dual server/client socket system. The system employs native URScript interface of the UR10 to communicate with the embedded position controller. This controller executes the motion of ARMM coil upon receiving a pose command, expressed as a position $\left(\mathbf{p}_{C} \in \mathbb{R}^{3}\right)$ and angleaxis orientation $\left(\boldsymbol{\theta}_{C} \in \mathbb{S}^{3}\right)$ of the tool centre-point $(\mathrm{TCP})$ frame of reference $(\{\mathcal{T}\})$ (Figure 2). This pose is retrieved as follows:

$$
\begin{aligned}
\mathbf{p}_{C} & =\mathbf{p}-z \mathbf{Z}_{C}, \\
\boldsymbol{\theta}_{C} & =\operatorname{acos}\left(\widehat{\mathbf{z}} \cdot \mathbf{Z}_{C}\right) N\left(\widehat{\mathbf{z}} \times \mathbf{Z}_{C}\right),
\end{aligned}
$$




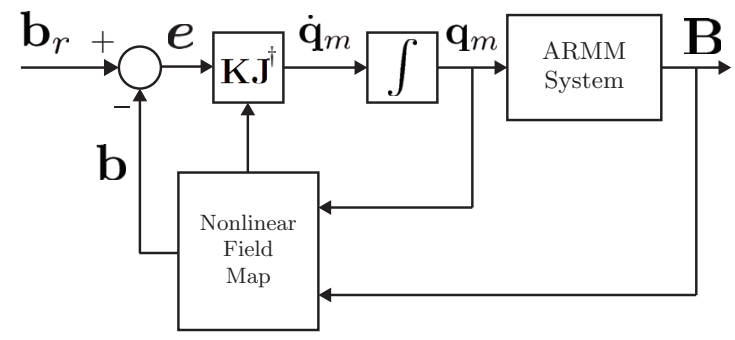

Figure 3.6: The ARMM (Advanced Robotics for Magnetic Manipulation) system employs an iterative field map to generate reference magnetic field and its gradient $\mathbf{b}_{r}=\left[\begin{array}{ll}B_{r} \quad \frac{\partial B_{r}}{\partial z}\end{array}\right]^{T}$ at $\mathbf{p}$. The information about the configuration $\left(\mathbf{q}_{m}=\left[\begin{array}{ll}z & I\end{array}\right]^{T}\right)$ of the system, as well as real-time measurements from magnetic field sensor are used to generate an error signal $\left(\mathbf{e} \in R^{2}\right)$. The error is minimized using a Jacobian inverse scheme, employing the derivation presented in (3.14). $\dagger$ denotes damped pseudo-inverse, used in place of regular matrix inverse for improved numerical stability [180]. The gain $\left(\mathbf{K} \in \mathbb{R}^{2 \times 2}: \mathbf{K}>0\right)$ of the scheme is tuned manually until satisfactory performance is achieved.

where $\widehat{\mathbf{z}}=\left[\begin{array}{lll}0 & 0 & 1\end{array}\right]^{T}$ and $N(\boldsymbol{\alpha})=\frac{1}{\|\boldsymbol{\alpha}\|} \boldsymbol{\alpha} \forall \boldsymbol{\alpha} \in \mathbb{R}^{3}$. The current in the coil is controlled directly using XEL-230-40 (Copley Controls, Boston, USA) amplifier connected via EtherCAT. (Figure 3.2).

\subsubsection{Performance and Limitations of ARMM}

Several factors affect the effective workspace of the system, in terms of its capabilities of generating desired magnetic torques and forces. Our actuation method assumes that for every $\mathbf{p}$ within the effective workspace of the ARMM, the coil is capable of moving within a distance $(z)$ along any $\mathbf{Z}_{\mathbf{c}}$. To make this assumption realistic, we impose bounds $\left(z \in\left[z_{\min }, z_{\max }\right]\right)$. The minimum bound $z_{\min }$ is set at $50 \mathrm{~mm}$ such that the position of the coil is always at a safe distance, since $\mathbf{p}$ is likely to be inside the body of a patient. The maximum bound $z_{\max }$ is selected arbitrarily at $300 \mathrm{~mm}$ as positioning the coil at larger distance from the body is impractical due to resulting very small magnitudes of the magnetic field. Furthermore, the realisation of the ARMM system imposes bounds on the maximum value of the current $(|I|<20 \mathrm{~A})$, due to the limitations of XEL-230-40.

Knowing these limits, the size of the effective workspace can be coarsely approximated. We define it as the collection of points for which the ARMM system can position its coil between $\left(z_{\min }\right)$ and $\left(z_{\max }\right)$ in any direction. The workspace, in which the UR10 can position its tool centre point (TCP) at any direction is a sphere of $1300 \mathrm{~mm}$. The face of the coil is at the distance of $217 \mathrm{~mm}$ from the TCP. Assuming $z_{\max }$ of $300 \mathrm{~mm}$, the effective workspace of the ARMM system can be approximated with a sphere with a radius of $783 \mathrm{~mm}$ ).

Nevertheless, these considerations are driven by a set of simplified assumptions. The actual effective workspace of the ARMM system depends on more factors, such as the presence of obstacles or the limitations of the kinematics of UR10. In order to define a procedure-specific workspace of the device, extensive kinematic analysis 
needs to be performed. Furthermore, by selecting a robotic arm with larger reach, the workspace can be easily scaled up to desired dimensions. We believe that this feature of the mobile coil systems will, in the future, motivate their extensive use in procedures requiring magnetic actuation.

The bounds relating to limitations of the ARMM system are imposed on $\mathbf{q}_{m}$ upon every iteration of the map. This guarantees that for every desired $\mathbf{b}$, the iterative map provides a configuration $\left(\mathbf{q}_{m}\right)$, which falls within the limits of the device, allowing for safe operation of ARMM. Nevertheless, by imposing these bounds, we expect its accuracy to be guaranteed only if $\mathbf{b}$ falls within a certain range of values. To quantify that range, we develop a simulation using a computational model of the ARMM coil, with $\eta$ being approximated using offline measurements. The results (Figure 3.7) confirm our expectations, thus the accuracy of the device must be taken into account, when employing ARMM system for the steering of flexible surgical instruments.

Furthermore, Figure 3.7 demonstrates that the ARMM system can generate fields of at least $20 \mathrm{mT}$ and gradients of more than $0.6 \mathrm{mT} / \mathrm{mm}$, similar to the ones used in previous studies on magnetic catheters [95,136,155,179]. These values by no means mark the upper limit of the system, as at a very close distances $(z<50 \mathrm{~mm})$ the maximum field generated by the ARMM exceeds $80 \mathrm{mT}$. Thus, if collision avoidance algorithms are employed to safely keep the coil close to the skin of the patient at all times, actuation conditions comparable with commercial systems can be achieved [116,134]

\subsubsection{Experimental Validation}

We validate the implementation of the iterative map experimentally (Figure 3.8). We employ the ARMM system to generate a series of prescribed field/gradient trajectories for a given location ( $\mathbf{p})$. The magnetic field generated by the system is measured using a Senis 3MH3A-500MT (Senis AG, Baar, Switzerland) Teslameter, while the gradient is measured indirectly by registering the force $\left(\mathbf{F}_{\mu}\right)$ acting on a permanent magnet with known magnetic dipole moment $(\boldsymbol{\mu})$. The magnet is attached to a K3D40 force sensor (Meßsysteme AG, Henningsdorf, Germany). The force measurements are processed using (3.2) to obtain the final value of magnetic gradient.

Figure 3.9 presents the results from three representative trajectories. The data shows good correspondence between the reference and actual values of the field and gradient, except for a region in state trajectory 1, where the actual field diverges from the reference due to the solution exceeding the bound of the current (-20 A, visible on the bottom graph). This state trajectory has been selected purposefully, in order to demonstrate the performance of the map in such situations. Remarkably, the generated gradient still corresponds well to the prescribed value (average error $5.57 \%$ ).

Excluding the out-of-bounds region from the analysis, the average percentage error across all trajectories is $2.34 \%$ for the magnetic field and $7.2 \%$ for the magnetic field gradient. There is no significant difference between the errors in the linear $(|I|<15 \mathrm{~A})$ and non-linear $(|I|>15 \mathrm{~A})$ regions of coil operation. Thus, the generation of prescribed magnetic field and gradients with independently con- 


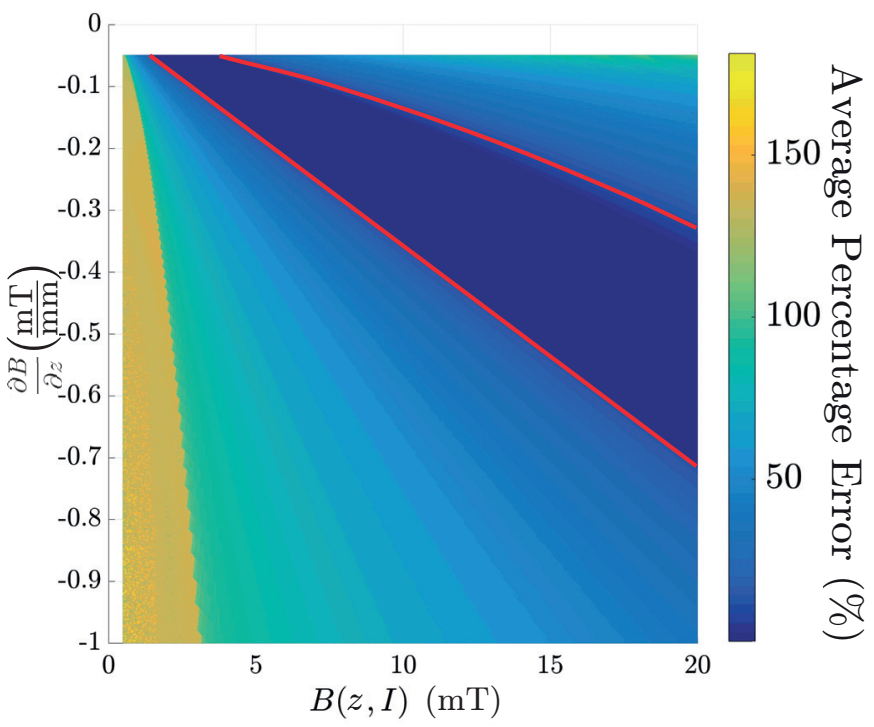

Figure 3.7: Results of the simulation testing the accuracy of the iterative field map (presented in Section 3.3) for different magnetic field/gradient setpoints $\left(\mathbf{b} \in \mathbb{R}^{2}\right)$. The map is solved for $\mathbf{a}$ total of $10^{6}$ different combination of $\mathbf{b}$ of the magnetic field $(B(z, I) \in \mathbb{R})$ and gradient $\left(\frac{\partial B}{\partial z}\right)$. The solution obtained for each $\mathbf{b}$ is fed to the forward model $(3.12-3.13)$ to calculate the actual field and gradient it produces. The percentage error between the desired and actual $\mathbf{b}$ is calculated. The results show that imposing the bounds on the set of possible $\mathbf{q}_{m}$, does not result in a significant change to the subset $\left(\mathbf{q}_{g} \subset \mathbf{q}_{m}\right)$, which can be generated by ARMM system with errors below $10 \%$ (marked by red lines). We expect that this is due to the effect of using a damped pseudo-inverse. However, detailed proof of that lies beyond the scope of this study.

trolled magnitudes can be achieved using the iterative map to drive the ARMM system.

\subsection{Conclusions and Future Work}

In this chapter, the problem of achieving magnetic actuation of flexible surgical instruments (such as endovascular catheters) in large workspaces is addressed by development of the ARMM system. By employing a mobile coil approach, the ARMM system provides magnetic actuation in a large spherical workspace. The maximum radius of that workspace $(1300 \mathrm{~mm})$ allows for steering magneticallyactuated catheters in every point of human body without the need of repositioning the patient. Moreover, the ARMM system employs a UR5 robotic arm which is used as an assistive device holding an ultrasound probe intended to be used as a primary medical imaging modality. The electromagnetic coil of the ARMM system employs a Vacoflux 50 ferrous core, and is designed using an optimization procedure to maximize the magnetic field in the target region under payload and heat dissipation constraints. The cost function used in our procedure optimizes the geometry of the coil for the purpose of our novel magnetic actuation 


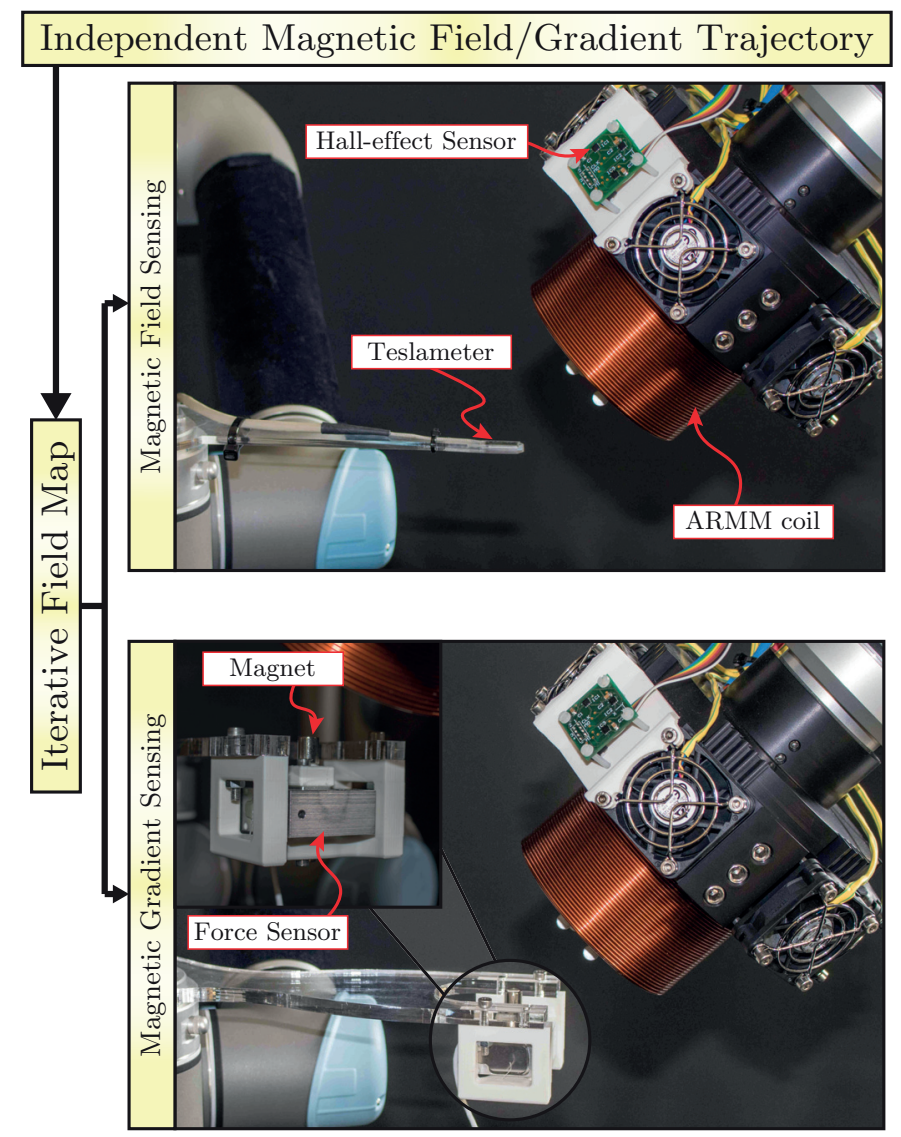

Figure 3.8: The setup used to validate the iterative map proposed in Section 3.3. In this experiment, the ARMM (Advanced Robotics for Magnetic Manipulation) system is used to generate prescribed magnetic field/gradient state trajectories for a selected point $\left(\mathbf{p} \in \mathbb{R}^{3}\right)$. Initially, a Senis 3MH3A-500MT (Senis AG, Baar, Switzerland) teslameter is used to sense magnetic field at $\mathbf{p}$ for the duration of the reference state trajectory. Following that, the teslameter is replaced by a permanent dipole with known magnetic dipole moment $(\boldsymbol{\mu})$ attached to a K3D40 force sensor (Meßsysteme AG, Henningsdorf, Germany) and the state trajectory is repeated. The measurements from the force sensor are processed to recreate the magnetic field gradient generated by ARMM. Both sensors are precisely positioned at $\mathbf{p}$ using the UR5 robotic arm (Universal Robots, Odense, Denmark) to ensure that the measurements are taken in the same location.

strategy. This strategy involves using the robotic arm to follow the position of the device in real-time, which simplifies the magnetic field models and the resulting actuation.

The results of coil characterization indicate that the optimization procedure is effective in generating coil geometries resulting in large magnetic fields, suitable for our actuation strategy. In fact, the predictions of finite-element model used in the optimization routine underestimate the field generated by the real device due to lack of accurate material model for a Vacoflux ferrous core. Since the Vacoflux core of the coil used in the ARMM system exhibits saturation within 

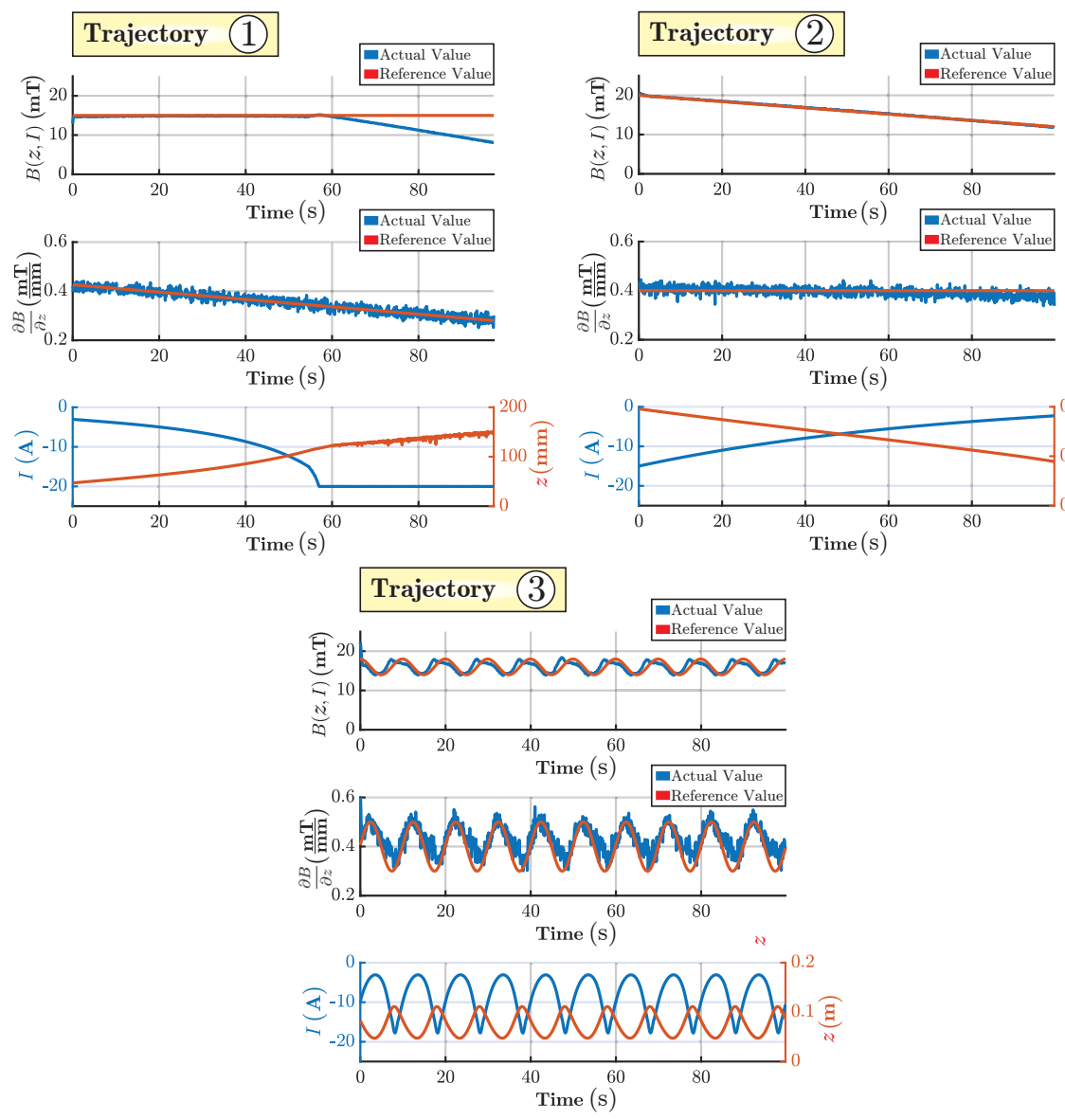

Figure 3.9: The results of the experiment presented in Figure 3.8. The iterative map is used to generate magnetic field/gradients following three reference state trajectories. State trajectory (1) demonstrates the effect of bounds of current $(I)$ on the accuracy of the solution. Disregarding that part of the state trajectory, the average error across all state trajectories is $2.34 \%$ for the magnetic field and $7.20 \%$ for the magnetic field gradient.

the operational range, the resulting relationship between the coil current and the magnetic field becomes non-linear. This phenomenon makes precise magnetic actuation challenging. We address this issue by developing a novel iterative map technique. This technique makes use of the overactuation of the ARMM system, allowing to generate magnetic fields and gradients with independently prescribed magnitudes. Furthermore, it uses a hall-effect sensor to account for the saturation of the Vacoflux core. The technique is validated, showing an average error of $2.34 \%$ for magnetic field and $7.20 \%$ for magnetic gradient.

The next steps in our work involve demonstrating the clinical feasibility of the ARMM system in steering magnetically-actuated catheters. We realise these experiments in Chapter 6. There, we develop a control strategy, which enables magnetic actuation of catheters in clinically-relevant tasks under the guidance of scanning ultrasound. 



\section{Part II}

\section{Small but Sassy: Mesoscale Magnetic Catheters}





\section{Preface}

Research undertaken in the previous part results in versatile technology for actuation of magnetic catheters. With both BigMag and the ARMM system in place, concepts for novel functionalised catheters can be experimentally investigated. Despite their small size and relatively simple structure, magnetic catheters have capabilities of exhibiting immensely complex mechanical behaviour, determined by their structure and material composition. In this part, we descend to mesoscale to harness this behaviour through design and automation. In a series of studies, we present prototype robotic magnetic catheters. We employ magnetic elements in our designs, enabling each prototype to execute a clinically-relevant task. Using the principles of continuum robotics, we automate our prototypes, providing means to track, model and control their behaviour during operation.

The most important feature of every flexible catheter is its ability to be navigated to a target location within the body, following a position trajectory specified by a clinician. In Chapter 4 we present a method, in which the task of position control of a catheter tip can be performed autonomously using a vision-based system for tracking and the BigMag mobile coil array for actuation. We apply a reducedorder pseudo-rigid-body (PRB) model to represent the behaviour of a catheter with a permanent magnet at its tip. Using quasi-static assumption, we derive an inverse-model controller, relating the tip velocity to the rate of change of magnetic torques applied by the BigMag system. The configuration of the PRB model is obtained by processing point-cloud silhouettes of the catheter using a novel algorithm. Our method is tested experimentally, steering a prototype catheter across a series of pre-defined trajectories.

Use of magnetic actuation in catheter design allows for straightforward realisation of otherwise challenging functionalities. We demonstrate that in Chapter 5, presenting a tentacle catheter for underactuated grasping. The tentacle catheter has a body made out of compliant polymer with a permanent magnet at its tip. Using external magnetic actuation provided by BigMag, the catheter body can be wrapped around a target forming a loop. A miniaturized electromagnetic coil, located at the proximal end of the catheter is used to stabilise the looped shape providing form closure sufficient for object manipulation. The prototype is characterized with respect to the maximum force available for manipulation. Additionally, its operation is demonstrated in an experiment involving grasping and displacement of porcine tissue.

The elaboration of know-how generated in this part of the dissertation is presented in Chapter 6. Here, a complex magnetic catheter for targetted drug delivery is developed and integrated within a clinically-relevant workflow realised 
with ARMM system. The catheter is used to deliver axially-magnetised cylindrical capsules, which contain the drug and hard magnetic material embedded in biodegradable matrix. A capsule is located in the distal compartment of the catheter tip and held in place by a miniaturized electromagnet located proximally. The total dipole moment of the two elements is actuated using the ARMM system, steering the catheter to the target region. There, the capsule is ejected from the tip and steered as a projectile. For the purpose of catheter steering, we rework the PRB modelling framework initiated in Chapter 4, significantly expanding it to account for the kinematics of the ARMM system. The point cloud algorithm used to extract the PRB configuration is integrated within custom-built volumetric ultrasound scanner. Furthermore, we demonstrate how, by exploiting the overactuation of the ARMM system, the position trajectory of the capsule upon release from the catheter can be controlled. The proposed drug delivery procedure is validated in a proof-of-concept experiment 


\title{
Chapter 4
}

\section{Vision-Based 3D Steering of Magnetic Catheter Using BigMag}

\begin{abstract}
Automated steering of endovascular catheters has a potential of improving the outcome of minimally invasive surgical procedures. Nevertheless, actuation, tracking and closed-loop position control of catheters remain a challenge. In this study, we present a modular framework for 3D position control of magneticallyactuated endovascular catheter. The catheter is fitted with a permanent magnet and deflected using externally-generated magnetic field provided by BigMag - an array of mobile electromagnets. Pseudo-rigid-body modelling is used to formulate an inverse-model closed-loop position controller of the catheter. The shape feedback is reconstructed from a 3D point cloud of catheter silhouette, obtained using stereo vision. The accuracy of the shape reconstruction algorithm is $0.59 \mathrm{~mm}$. The magnetically-actuated catheter is steered across a series of trajectories with a maximum reported catheter deflection of $68.43^{\circ}$ and maximum tip speed of $5 \mathrm{~mm} / \mathrm{s}$. Across all trajectories, the best control performance metrics are the mean error of $0.57 \mathrm{~mm}$ and the RMS error of $0.77 \mathrm{~mm}$.
\end{abstract}

Adapted from:

J. Sikorski, A. Denasi, G. Bucchi, S. Scheggi, S Misra. Vision-based 3-D control of magnetically actuated catheter using BigMag - an array of mobile electromagnetic coils, IEEE/ASME Transactions on Mechatronics vol. 24, no. 2, pp. 505-516, 2019 


\subsection{Introduction}

Endovascular catheters are an important class of surgical devices used for minimally invasive surgery (MIS) within the human cardiovascular system [195]. These catheters are inserted through small incisions in limbs and navigated through blood vessels to reach more centrally located target sites, such as the brain or the heart. Conventional MIS navigation of endovascular catheters is manual, which significantly limits its precision, thus requiring skilled personnel in order to ensure patient safety. By employing robotic technology, steering of endovascular catheters can be facilitated. This has already been demonstrated in several standard MIS procedures [65,68].

Steerable catheters can become versatile tools, capable of reaching any desired target within the cardiovascular system. Nevertheless, ensuring reliable actuation and control of such devices still poses a significant challenge. From the perspective of robotics, catheters are classified as continuum manipulators (CM), that is manipulators having a hyper-redundant structure of continuously bending elastic elements. In most applications CM are actuated using tendons or guide-wires, and modelled as arcs with constant curvature $[81,170,196]$. Due to the small size of the catheters and their non-homogeneous structural compositions, the limitations of this solution are pronounced. The accuracy of tendon steering is limited due to actuation hysteresis and inaccuracies of constant curvature approximation. Furthermore, significant friction experienced by the tendons can lead to their failure, threatening the safety of the patient [172].

The first suggestion that magnetic interaction can be used to remotely steer CM was postulated over 60 years ago [114]. Magnetic CM can be made safer, and smaller than the ones driven by tendons, due to their simpler structure involving no moving parts. Therefore, this actuation technique is well-suited for catheters.

Magnetically-actuated catheters presented in the literature can be broadly divided into passive or active devices [134]. Active magnetic catheters are fitted with microcoils, which generate variable magnetic dipole moments. If located in a large static external magnetic field of a magnetic resonance scanner (MRS), such catheters can be deflected by controlling the electric currents flowing through microcoils $[197,198]$. On the contrary, the passive catheters are fitted with permanent magnets with constant magnetic dipole moments, and steered by varying the external magnetic field generated by custom, dedicated systems [106,117].

Evaluating both classes of devices from a practical point of view, passive magnetic catheters are better suited for automated endovascular procedures. If the dipole provided by a microcoil is aligned with the field of MRS, the catheter loses a degree of freedom (DoF) [199]. Moreover, the microcoils are expensive and difficult to manufacture, and their heat dissipation causes safety concerns [200]. Passive catheters display none of these limitations. They can be readily assembled using inexpensive and accessible elements. Furthermore, they allow for 5DoF actuation irrespective of their pose within the workspace.

Magnetic guidance systems employing passive catheters are already available commercially, yet they rely entirely on open-loop control performed by the clinician [201]. Catheter steering using closed-loop position control has so far received limited attention. 2D steering has been approached in several studies, using linear 
control with decoupling $[95,155,174]$. Nevertheless, none of these techniques were extended to $3 \mathrm{D}$ position control.

The first successful attempt thereat has been presented recently by Edelmann et al. [116]. This technique employs a 3D non-linear position controller, based on the geometrically exact Cosserat rod model. However, this approach has been demonstrated only for small angle deflections and slow effective motion speed of the catheter tip. This is due to limitations caused by the low bandwidth of the magnetic actuation system used, inadequate shape feedback and computational complexity of the rod model.

The closed-loop steering of the $\mathrm{CM}$ is enabled by feedback providing the information about the shape of the device in real-time. Medical imaging modalities, such as fluoroscopy or ultrasound, are preferred for that purpose since they are capable of providing information in reference to tissues inside the body. Imaging data is usually available in the form of $3 \mathrm{D}$ point clouds. Several techniques to reconstruct the shape of a CM from 3D point clouds have already been presented. However, their limitations, such as low computational performance or inability to track CM undergoing larger bends suggest the need for an alternative [156,157].

In this chapter we present a modular framework for modelling, tracking and control of passive magnetic catheters (Figure 4.1). The magnetic catheter is represented using a computationally efficient pseudo-rigid-body model (PRBM). We employ the elements of two existing quasi-static models based on this approach to derive an analytical (i.e. computationally fast) formulation of manipulator Jacobian [74, 199]. Consequently, our PRBM is used to derive a non-linear inverse model controller for steering of a magnetic catheter tip in $3 \mathrm{D}$ space. The proposed controller uses shape feedback from a 3D point-cloud, processed using a novel reconstruction technique. This technique offers a very good computational performance, being in the same time suitable for processing point-clouds from various imaging modalities.

The catheter is actuated using BigMag, an array of mobile electromagnetic coils, which offers magnetic actuation in a large workspace with high bandwidth [179]. In this chapter we use the field map presented in Chapter 2, demonstrating how to employ BigMag for accurate actuation of magnetic catheter. To the authors' best knowledge, BigMag is the first array of mobile coils used for that purpose.

The rest of this chapter is structured as follows. In Section 4.2, we present a PRBM of a passive magnetic catheter. The shape reconstruction algorithm is given in Section 4.3. The full control framework is presented in Section 4.4. Finally, Section 4.5 details the experiments and discusses the results, validating our steering technique, and Section 4.6 summarises the chapter and gives recommendations for further studies.

\subsection{Modelling a magnetic catheter}

Magnetic catheter exhibits highly non-linear dynamical behaviour. The modelling of this behaviour is impractical for high bandwidth position control. Therefore, we propose to approximate catheter mechanics with a computationally fast, quasistatic PRBM proposed by Greigarn and Çavuşoğlu in in [199]. This model de- 


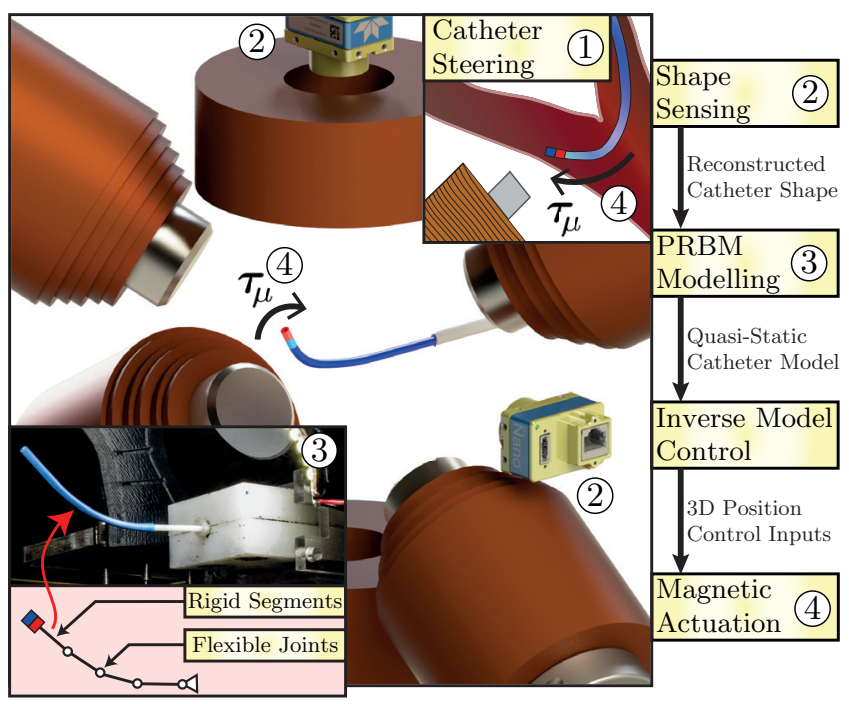

Figure 4.1: In this integrative study we present a framework for 3D position control of magnetically-actuated catheter (1). An iterative algorithm tracks the shape of the catheter using 3D point clouds provided by stereo vision system (2). The reconstructed shape is approximated by pseudo-rigid-body model (PRBM) (3). We use PRBM to formulate an inverse model controller for 3D position control of the catheter employing magnetic torques $\left(\tau_{\mu}\right)$ (4). The torques are generated using BigMag - a mobile coil array.

scribes the shape of the catheter under the influence of an external magnetic torque and gravity forces. Moreover, it provides an analytical formulation of body manipulator Jacobian, and thus can be effectively applied in real-time for catheter steering.

Let us start by considering the shape of a magnetic catheter (Figure 4.2), which can be described by a continuous, smooth function $\left(\mathbf{P}(s): \boldsymbol{\Omega}_{p} \mapsto \boldsymbol{\Omega}_{w}\right)$, such that

$$
\begin{aligned}
& \boldsymbol{\Omega}_{p}=\{s \in \mathbb{R} \mid 0 \leq s \leq l\}, \\
& \boldsymbol{\Omega}_{w}=\{x, y, z \in \mathbb{R} \mid \underline{x} \leq x \leq \bar{x}, \underline{y} \leq y \leq \bar{y}, \underline{z} \leq z \leq \bar{z}\},
\end{aligned}
$$

where $₫$ and $\mp$ are the limits of the workspace for $\star=\{x, y, z\}$. In (4.1), the parameter $(s)$ defines the position along the length of the catheter, spanning between base point $(s=0)$ and the tip $(s=l)$. Consequently, $l \in \mathbb{R}^{+}$is the total length of the catheter and $\boldsymbol{\Omega}_{w}$ is its workspace defined as a subset of Cartesian space.

The magnetic catheter is connected to a guiding sheath (Figure 4.2), used for insertion. The stiffness of the sheath is much higher than the one of the catheter, therefore we neglect its role in the bending of the catheter. Using PRB discretization technique, we can represent the flexible catheter by $(n)$ rigid segments. The segments are described by reference frames $\left(\left\{\Psi_{s}^{i}\right\}, i=1, \ldots, n\right)$, each located at a point $\left(\mathbf{p}_{i}=\mathbf{P}\left(i \frac{l}{n}\right) \in \mathbb{R}^{3}\right)$. Additionally, frame $\left\{\Psi_{s}^{0}\right\}$ located at $\mathbf{p}_{0}$ denotes the base of the catheter.

The catheter is assumed to be an externally-loaded incompressible beam. The torsion and buckling of the catheter are neglected, as the torsional stiffness of the 


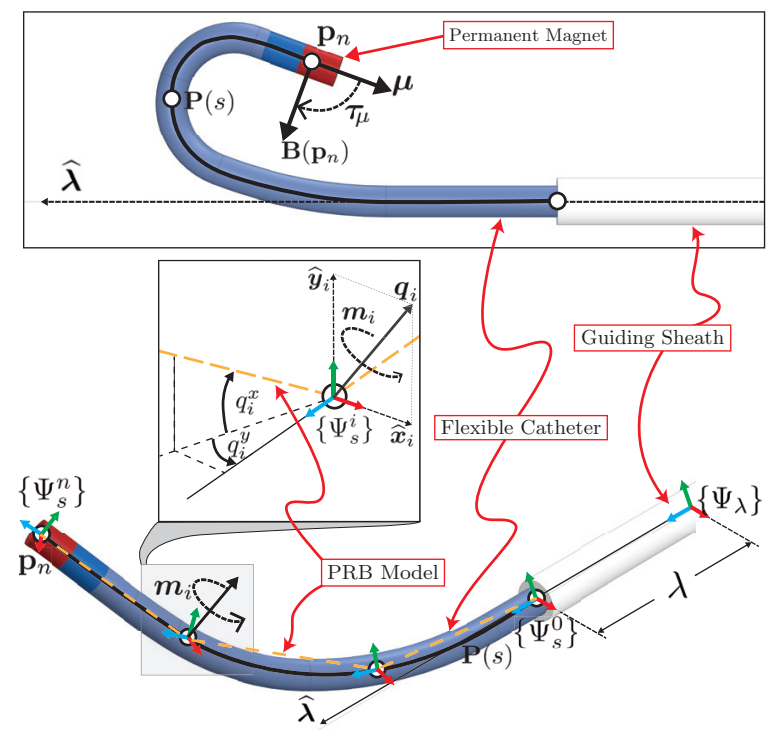

Figure 4.2: Top: Magnetic catheter has a single magnet at its tip, represented by magnetic dipole moment $(\boldsymbol{\mu})$. In presence of external magnetic field $\left(\mathbf{B}\left(\mathbf{p}_{n}\right)\right)$ the catheter is bending due to magnetic torque $\left(\boldsymbol{\tau}_{\mu}\right)$. Its shape can be represented by a $3 \mathrm{D}$ curve $(\mathbf{P}(s))$ Bottom: The kinematics of bending flexible catheter can be approximated using a quasi-static pseudo-rigidbody model. The manipulator is represented with a series of discrete rigid segments $(i \in[1 ; n])$ connected by $2 \mathrm{DoF}$ elastic joints. Each elastic joint $(i)$ is affected by the corresponding joint torque $\left(\mathbf{m}_{i} \in \mathbb{R}^{3}\right)$. The deflection due to that torque is parametrized by a set of 2 angles $\left(q_{i}^{x}, q_{i}^{y}\right)$.

catheter structure is usually much higher than the bending stiffness, and the buckling is unlikely to occur without external contacts, the modelling of which remains outside the scope of this work. The shape $(\mathbf{P}(s))$ of the catheter is determined by a mechanical load applied along its length, which follows from Euler-Bernoulli beam theory. We approximate this total load by a point wrench $\left(\mathbf{W} \in \mathbb{R}^{6}\right)$, acting at the tip of the catheter $\left(\mathbf{p}_{n}=\mathbf{P}(l) \in \mathbb{R}^{3}\right)$. W is defined in body reference frame and comprises of a magnetic force $\left(\mathbf{F}_{\mu} \in \mathbb{R}^{3}\right)$ and a magnetic torque $\left(\boldsymbol{\tau}_{\mu} \in \mathbb{R}^{3}\right)$. Both $\mathbf{F}_{\mu}$ and $\boldsymbol{\tau}_{\mu}$ are exerted on the catheter due to the interaction of the dipole moment $\left(\boldsymbol{\mu} \in \mathbb{R}^{3}\right)$ located at the catheter tip $\left(\mathbf{p}_{n}\right)$ with the external magnetic field $\left(\mathbf{B}\left(\mathbf{p}_{n}\right) \in \mathbb{R}^{3}\right)$ as described by (1.1).

We assume the catheter to be in a reference configuration when $\mathbf{W}=\mathbf{0}_{6 \times 1}$. Given the insertion axis $\left(\hat{\lambda} \in \mathbb{R}^{3}\right)$ passing through the base of the catheter $\left(\mathbf{p}_{0}=\mathbf{P}(0) \in \mathbb{R}^{3}\right)$, the shape of the manipulator at the reference configuration is a straight line $(\mathbf{P}(s)=s l \widehat{\boldsymbol{\lambda}})$.

In PRBM approximation, the rigid segments representing the catheter are connected in series by a total of $n$ 2DoF flexible, revolute joints (Figure 4.2). The total rotation of joint $i$ is expressed with respect to frame $\left(\left\{\Psi_{s}^{i-1}\right\}\right)$ as $\mathbf{q}_{i}=\mathbf{w}_{i}^{x} q_{i}^{x}+\mathbf{w}_{i}^{y} q_{i}^{y} \in \mathbb{R}^{3}$, where $q_{i, x}, q_{i, y} \in \mathbb{S}$ are joint variables, $\mathbf{w}_{i}^{x}, \mathbf{w}_{i}^{y} \in \mathbb{R}^{3}$ are instantaneous joint axes. If $q_{i}=\sqrt{q_{i, x}^{2}+q_{i, y}^{2}}, \mathbf{w}_{i}^{x}, \mathbf{w}_{i}^{y} \in \mathbb{R}^{3}$ are derived as 
follows:

$$
\begin{aligned}
& \mathbf{w}_{i}^{x}=\left[\begin{array}{c}
1-\frac{q_{i, y}^{2}}{q_{i}^{2}}\left(1-\frac{\sin q_{i}}{q_{i}}\right) \\
\frac{q_{i, x} q_{i, y}}{q_{i}^{2}}\left(1-\frac{\sin q_{i}}{q_{i}}\right) \\
-\frac{q_{i, y}^{2}}{q_{i}^{2}}\left(1-\cos q_{i}\right)
\end{array}\right] \quad \mathbf{w}_{i}^{y}=\left[\begin{array}{c}
\frac{q_{i, x} q_{i, y}}{q_{i}^{2}}\left(1-\frac{\sin q_{i}}{q_{i}}\right) \\
1-\frac{q_{i, x}^{2}}{q_{i}^{2}}\left(1-\frac{\sin q_{i}}{q_{i}}\right) \\
\frac{q_{i, x}^{2}}{q_{i}^{2}}\left(1-\cos q_{i}\right)
\end{array}\right] \quad \text { for } q_{i} \neq 0 \\
& \mathbf{w}_{i}^{x}=\left[\begin{array}{lll}
1 & 0 & 0
\end{array}\right]^{T} \quad \mathbf{w}_{i}^{y}=\left[\begin{array}{lll}
0 & 1 & 0
\end{array}\right]^{T} \quad \text { for } q_{i}=0
\end{aligned}
$$

Collecting the joint variables, we define the total manipulator configuration as follows:

$$
\mathbf{q}=\left[\begin{array}{lllll}
q_{1, x} & q_{1, y} & \ldots & q_{n, x} & q_{n, y}
\end{array}\right]^{T} \in \mathbb{R}^{2 n} .
$$

The pose of each frame $\left(\left\{\Psi_{s}^{i}\right\}\right)$ with respect to $\left(\left\{\Psi_{s}^{0}\right\}\right)$ is represented by a homogeneous transformation $\left({ }_{i}^{0} \mathbf{H}(\mathbf{q})=\left[\begin{array}{cc}\mathbf{R}_{i}(\mathbf{q}) & \mathbf{p}_{i}(\mathbf{q}) \\ \mathbf{0}_{1 \times 3} & 1\end{array}\right] \in \mathrm{SE}(3)\right)$. Furthermore, if we define a twist associated with each joint $(i)$ as follows:

$$
\widehat{\boldsymbol{\xi}}_{i}=\left[\begin{array}{cc}
S\left(\mathbf{q}_{i}\right) & S\left(\mathbf{p}_{i-1}(\mathbf{0})\right) \mathbf{q}_{i} \\
\mathbf{0}_{1 \times 3} & 0
\end{array}\right] \in \operatorname{se}(3),
$$

the transformation $\left({ }_{i}^{j} \mathbf{H}(\mathbf{q}) \in \mathrm{SE}(3), j=0, \ldots, i-1\right)$ between any two frames $\left(\left\{\Psi_{s}^{j}\right\}\right)$ and $\left(\left\{\Psi_{s}^{i}\right\}\right)$ can be calculated from the following product of exponentials:

$$
{ }_{i}^{j} \mathbf{H}(\mathbf{q})=e^{\widehat{\boldsymbol{\xi}}_{j+1}} \ldots e^{\widehat{\boldsymbol{\xi}}_{i}}{ }_{i}^{j} \mathbf{H}(\mathbf{0})
$$

The PRBM technique represents the internal compliance of the flexible catheter using torsional springs located at each joint $(i)$. Due to this compliance, the particular configuration $(\mathbf{q})$ of a catheter depends on the effect of tip wrench on PRBM joints (Figure 4.2). This effect is represented by joint torques $\left(\mathbf{m} \in \mathbb{R}^{2 n}\right)$. Hence, the joint-space equation of motion of the catheter takes the following form:

$$
\mathbf{m}=\mathbf{C q}+\mathbf{Z}(\mathbf{q}) \dot{\mathbf{q}}+\mathbf{g}(\mathbf{q})
$$

where $\mathbf{C} \in \mathbb{R}^{2 n \times 2 n}$ is a positive-definite diagonal compliance matrix, $\mathbf{Z}(\mathbf{q}) \in$ $\mathbb{R}^{2 n \times 2 n}$ is the positive-semidefinite viscous damping matrix and $\mathbf{g}(\mathbf{q}) \in \mathbb{R}^{2 n}$ is the vector of moments due to gravity. The entries of $\mathbf{C}$ represent the PRBM torsional spring constants, and depend on the flexural rigidity of the catheter as shown in our previous work [74].

Using the principle of virtual work, the body manipulator Jacobian $\left(\mathbf{J}_{b}(\mathbf{q}) \in \mathbb{R}^{6 \times 2 n}\right)$ is used to relate tip wrench $(\mathbf{W})$, defined as in (1.1), and the joint torques $(\mathbf{m})$. We calculate $\mathbf{J}_{b}(\mathbf{q})$ using the derivation proposed by Greigarn 
and Çavuşoğlu [199]. If we define the transformed twists $\left(\boldsymbol{\xi}_{i, x} \boldsymbol{\xi}_{i, y} \in\right.$ so(3)) for x and $\mathrm{y}$ of the PRB joint $(i)$ as follows:

$$
\begin{aligned}
& \boldsymbol{\xi}_{i, x}=\left[\begin{array}{cc}
\mathbf{R}_{i}^{T}(\mathbf{q}) & -\mathbf{R}_{i}^{T}(\mathbf{q}) S\left(\mathbf{p}_{i}(\mathbf{q})\right) \\
0 & \mathbf{R}_{i}^{T}(\mathbf{q})
\end{array}\right]\left[\begin{array}{c}
-\mathbf{w}_{i}^{x} \times \mathbf{p}_{i}(0) \\
\mathbf{w}_{i}^{x}
\end{array}\right], \\
& \boldsymbol{\xi}_{i, y}=\left[\begin{array}{cc}
\mathbf{R}_{i}^{T}(\mathbf{q}) & -\mathbf{R}_{i}^{T}(\mathbf{q}) S\left(\mathbf{p}_{i}(\mathbf{q})\right) \\
0 & \mathbf{R}_{i}^{T}(\mathbf{q})
\end{array}\right]\left[\begin{array}{c}
-\mathbf{w}_{i}^{y} \times \mathbf{p}_{i}(0) \\
\mathbf{w}_{i}^{y}
\end{array}\right],
\end{aligned}
$$

then the body Jacobian $\left(\mathbf{J}_{b}(\mathbf{q})\right)$ is

$$
\mathbf{J}_{b}(\mathbf{q})=\left[\begin{array}{lllll}
\boldsymbol{\xi}_{1, x} & \boldsymbol{\xi}_{1, y} & \ldots & \boldsymbol{\xi}_{n, x} & \boldsymbol{\xi}_{n, y}
\end{array}\right] .
$$

The final model of the catheter is developed by combining (1.1) and (4.8). The gravity is modelled by a point load $\left(\mathbf{F}_{g} \in \mathbb{R}^{3}\right)$ located at the tip $\left(\mathbf{p}_{n}\right)$ of the catheter. We consider it to be the force due to the weight of the dipole (which has a much higher mass than the flexible tubing). Hence, the final static equilibrium equation is as follows:

$$
\mathbf{J}_{b}^{T}(\mathbf{q}) \mathbf{W}=\mathbf{C q}-\mathbf{J}_{b}^{T}\left[\begin{array}{c}
\mathbf{R}_{n}^{T}(\mathbf{q}) \mathbf{F}_{g} \\
\mathbf{0}_{3 \times 1}
\end{array}\right] .
$$

Note that we neglect the weight of the catheter tubing, as it is an order of magnitude lower than $\mathbf{F}_{g}$. Moreover, the effect of the viscous damping $(\mathbf{Z}(\mathbf{q}))$ is disregarded, due to the quasi-static assumption.

Driving the catheter to the static equilibrium described by (4.12), requires an external system capable of precise generation of prescribed magnetic wrenches $(\mathbf{W})$. Usually, magnetic forces $\left(\mathbf{F}_{\mu}\right)$ with magnitudes sufficient for catheter steering are unachievable in large workspaces due to rapid decay of magnetic field gradients at a distance from the field source [174]. Consequently, $\mathbf{F}_{\mu}$ is neglected in our study, and thus the torques $\left(\tau_{\mu}\right)$ are selected as the sole control inputs for catheter steering. As follows from (1.1), these torques arise due to magnetic field $\left(\mathbf{B}\left(\mathbf{p}_{n}\right)\right)$ at the position of the catheter tip $\left(\mathbf{p}_{n}\right)$. In this chapter, we use the inverse map technique presented in Chapter 2 (Section 2.3) as a method a of generating such a field for any point inside the workspace of the BigMag system.

\subsection{Vision-based catheter shape sensing}

Shape sensing is a crucial technology for the successful control of magnetic catheters. As follows from (1.1), both the position $\left(\mathbf{p}_{n}\right)$ of the tip dipole, as well as its magnetic dipole moment $(\boldsymbol{\mu})$ must be known to actuate the tip with a prescribed virtual wrench. Moreover, since the research problem presented in this chapter involves the steering of the magnetic tip, its position $\left(\mathbf{p}_{n}\right)$ is also required as a feedback to close the control loop. Finally, since the PRB approximation is used to derive the manipulator Jacobian, the corresponding configuration of the catheter $(\mathbf{q})$ must also be tracked and reconstructed in real-time.

In this section, we approach those challenges by using a point cloud processing technique for shape feedback. The entire approach is modular: the model recovery 
technique can be applied to point clouds from different sources, including medical imaging modalities, such as fluoroscopy or 3D ultrasound. Consequently, we demonstrate how the variables needed for actuation and control of the catheter can be retrieved from the estimated shape $(\widetilde{\mathbf{P}}(s))$.

The task of the catheter reconstruction (see Figure 4.3) involves processing of a point cloud of size $\left(m \in \mathbb{Z}^{+}\right)$, defined as a set

$$
\mathbb{V}_{t}^{0}=\left\{\mathbf{v}_{i}^{0} \in \boldsymbol{\Omega}_{w}\right\}, i=1, \ldots, m,
$$

where each voxel $\left(\mathbf{v}_{i}^{0}\right)$ corresponds to a $3 \mathrm{D}$ point contained within the silhouette of the device. The shape estimate $(\widetilde{\mathbf{P}}(s))$ is recovered from $\mathbb{V}_{t}^{0}$ using an iterative Algorithm 1. The procedure begins with finding the origin of the catheter $\left(\widetilde{\mathbf{p}}_{0}\right)$. The algorithm searches for all voxels within a close vicinity of the initial guess of the origin $\left(\mathbf{p}_{0}^{g}\right)$, predefined by the user, and averages them to calculate $\widetilde{\mathbf{p}}_{0}$ (Algorithm 1, Lines 1-2). In our procedure the user makes the initial guess for the first frame. Consequently $\widetilde{\mathbf{p}}_{0}$ for the previous point cloud is used as a guess for the current one.

Once the location of catheter origin is established, an iterative fitting procedure extracts a set $\left(\widetilde{\mathbb{P}}=\left\{\widetilde{\mathbf{p}}_{j}\right\}, j=1, \ldots, \widetilde{n}\right)$ of points along $\widetilde{\mathbf{P}}(s)$. The distance between each $\widetilde{\mathbf{p}}_{j}$ and $\widetilde{\mathbf{p}}_{j+1}$ is fixed to $\frac{l}{\widetilde{n}}$. $\widetilde{\mathbf{p}}_{j}$ is found in a series of steps. First, the set $\left(\mathbb{V}_{c}^{j}\right)$ of all the voxels closer to $\widetilde{\mathbf{p}}_{j-1}$ than $\frac{l}{\widetilde{n}}$ is established (Algorithm 1, Line 3 ). The number $(\widetilde{n})$ is selected large enough, such that the shape of the catheter between $\widetilde{\mathbf{p}}_{j-1}$ and $\widetilde{\mathbf{p}}_{j}$ can be approximated with a straight line. The least-squares technique is used to find the direction $\widehat{\mathbf{d}}$ of that line in $3 \mathrm{D}$ space (Algorithm 1, Line 4; Figure 4.3). Since the desired distance to the next point is known, line equation is used to find $\widetilde{\mathbf{p}}_{j}$ (Algorithm 1, Line 6 ). Used voxels are excluded and the procedure repeats until the entire $\widetilde{\mathbb{P}}$ is found.

Since the location of each element of $\widetilde{\mathbb{P}}$ along $\widetilde{\mathbf{P}}(s)$ is known, these points are used to fit the final shape function. We approximate the catheter shape with a parametric 3D polynomial (Algorithm 1, Line 7). 3D polynomials offer accurate shape representation combined with computational efficiency. The coefficients of the polynomial are found solving another least squares problem (Algorithm 1, Line 8). The final shape of the device formed in this way (Figure 4.3) is used to calculate those parameters of the catheter, which are needed for closed-loop control. Given the estimated shape function $\widetilde{\mathbf{P}}(s)$, the PRB model is constructed as shown in Section 4.2. Frame $\left(\left\{\Psi_{s}^{0}\right\}\right)$ is positioned at the location of estimated catheter origin. For every 2DoF PRBM joint $(i)$ the parameters $\left(q_{i}^{x}\right),\left(q_{i}^{y}\right)$ are found as follows:

$$
q_{i}^{x}=-\operatorname{atan} 2\left({ }^{i-1} y_{i},{ }^{i-1} z_{i}\right), q_{i}^{y}=\operatorname{atan} 2\left({ }^{i-1} x_{i},{ }^{i-1} z_{i}\right),
$$

where

$$
\left[\begin{array}{llll}
{ }^{i-1} x_{i} & { }^{i-1} y_{i} & { }^{i-1} z_{i} & 1
\end{array}\right]^{T}={ }_{i-1}^{0} \mathbf{H}\left[\begin{array}{ll}
\mathbf{p}_{i}^{T} & 1
\end{array}\right]^{T} .
$$

The transformation matrix $\left({ }_{i-1}^{0} \mathbf{H} \in \mathrm{SE}(3)\right)$ is calculated using (4.7). The orientation of the magnetic tip, required for magnetic dipole moment estimation (and 


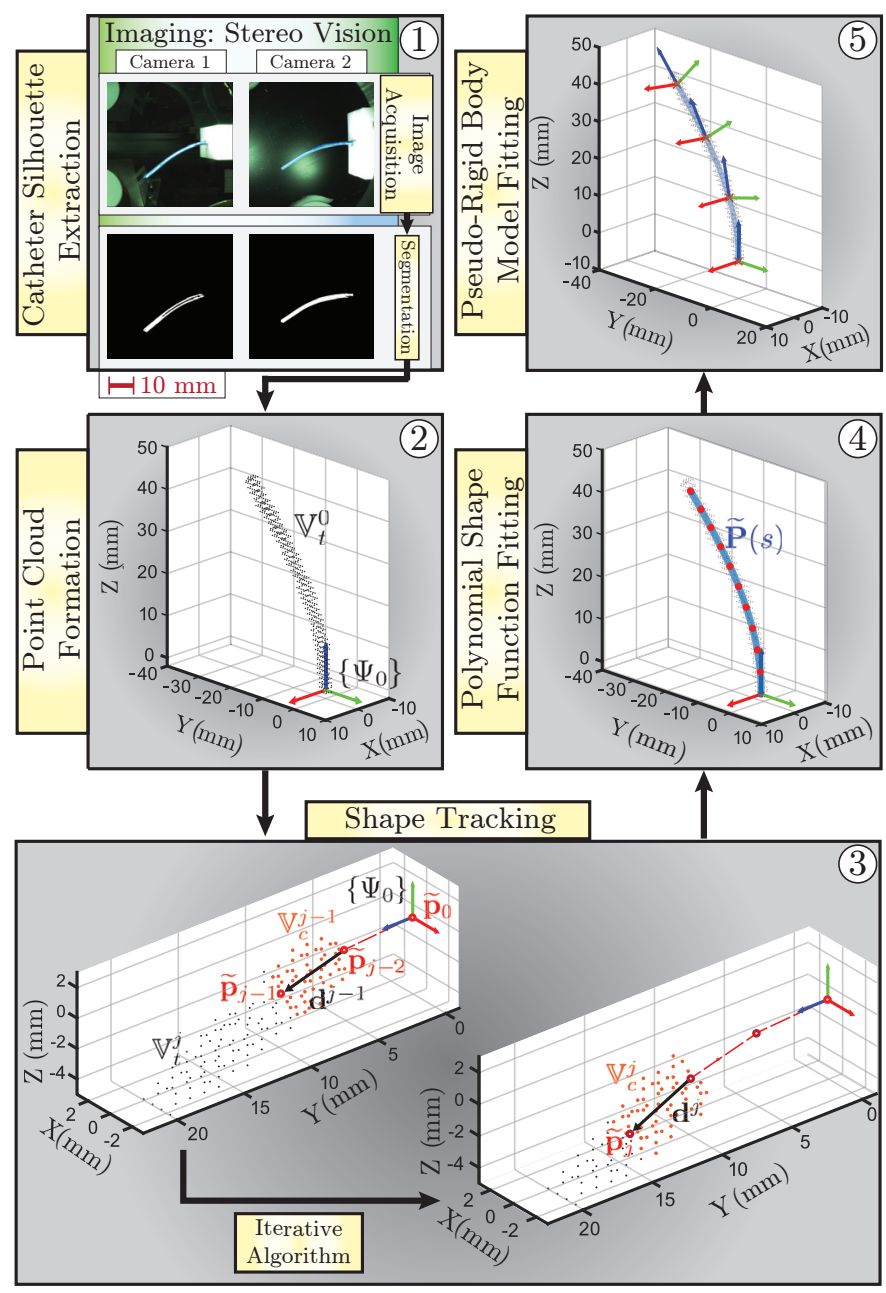

Figure 4.3: The vision-based catheter tracking is a modular pipeline process, which can be divided into five major steps. (1) The silhouette of the catheter is registered using stereo vision with two cameras. (2) The point cloud $\left(\mathbb{V}_{t}^{0}\right)$ with voxels describing catheter silhouette is formed for further processing. (3) An iterative algorithm computes a set of evenly spaced points $\left(\widetilde{\mathbb{P}}=\left\{\mathbf{p}_{j}\right\}\right)$ along the catheter shape. The fitting begins at the catheter origin $\left(\mathbf{p}_{0}\right)$. In each iteration the distance between $\mathbf{p}_{j-1}$ (established in previous iteration) and $\mathbf{p}_{j}$ is known. The subset $\left(\mathbb{V}_{c}^{j} \subset \mathbb{V}_{t}^{j}\right)$ comprising of voxels within that distance is used to determine direction $\left(\mathbf{d}^{j}\right)$ in which the $\mathbf{p}_{j}$ is located. Finally, $\mathbf{p}_{j}$ is calculated from $3 \mathrm{D}$ line equation, and voxels in $\mathbb{V}_{c}^{j}$ are excluded from further iterations. (4) The estimated catheter shape $(\widetilde{\mathbf{P}}(s))$ is approximated by a polynomial function fitted onto $\widetilde{\mathbb{P}}$. (5) This function is used for complete reconstruction of the pseudo-rigid-body model used for closed-loop control. Note: all reference frames have red, green and blue axes for $\mathrm{X}, \mathrm{Y}$ and $\mathrm{Z}$ respectively.

thus magnetic actuation) is computed using the normalised derivative of the shape function at the tip. The magnitude of the dipole $\left(\mu \in \mathbb{R}^{+}\right)$is assumed to be known beforehand. Hence, the total estimated tip dipole $\left(\boldsymbol{\mu}_{\text {est }} \in \mathbb{R}^{3}\right)$ becomes 


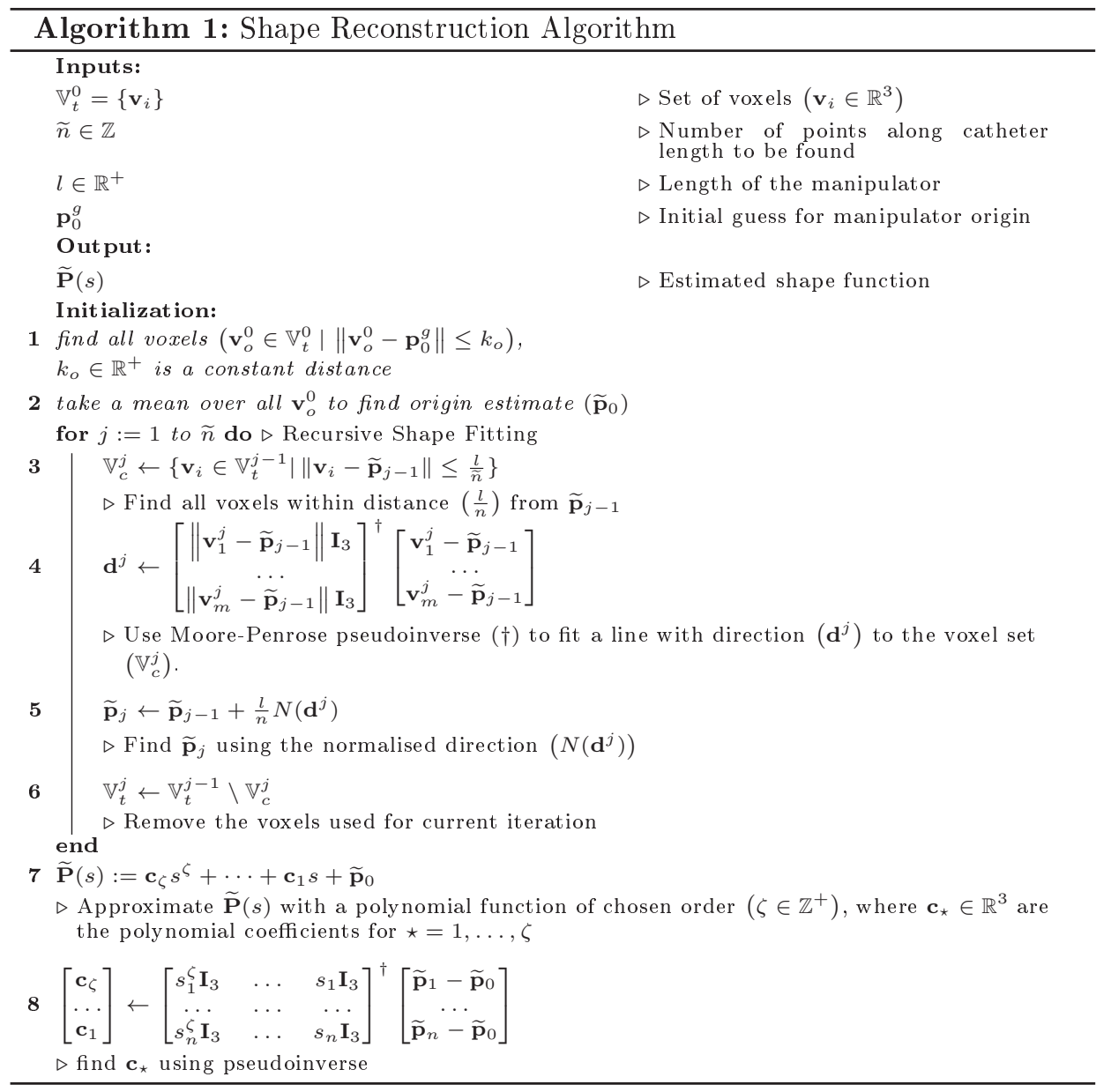

$$
\boldsymbol{\mu}_{e s t}=\mu N\left(\left.\frac{\mathrm{d} \mathbf{P}(s)}{\mathrm{d} s}\right|_{s=l}\right),
$$

where $N(\boldsymbol{\alpha})=\frac{\boldsymbol{\alpha}}{\|\boldsymbol{\alpha}\|}$ for any vector $(\boldsymbol{\alpha})$.

\subsection{Closed-Loop Steering of Magnetic Catheters}

In this section we propose a technique for closed-loop steering of magnetic catheters. The PRBM of a catheter introduced in Section 4.2 is used to derive a non-linear inverse model controller. The controller (Figure 4.4) is used in conjunction with feedback from visual tracking and reconstruction presented in Section 4.3 for real-time steering of magnetic catheter. 


\subsubsection{Inverse Model Control}

In the proposed steering technique, the catheter is actuated by an external magnetic torque $\left(\tau_{\mu}\right)$, changing its shape dynamically until it reaches a static equilibrium approximated by the PRBM configuration (4.12). Since the dynamic behaviour of the catheter moving towards the equilibrium exceeds the bandwidth of the available steering system, we disregard it. Therefore, we treat the static model (4.12) as a reduced-order model of the catheter, which can be used for controller design.

We begin the derivation of the controller reformulating the model (4.12). Since the gravity is represented by a single force acting on the catheter tip, it is useful to combine it with $\mathbf{W}$ into a single wrench describing the total external load on the catheter. However, a problem arises, since the gravitational force vector $\left(\mathbf{F}_{g}\right)$ becomes dependent on the manipulator configuration (q), if expressed in body frame of reference $\left(\left\{\Psi_{s}^{n}\right\}\right)$. In spite of that, if we transform the body manipulator Jacobian $\left(\mathbf{J}_{b}(\mathbf{q})\right)$ such that

$$
\mathbf{J}_{a}(\mathbf{q})=\left[\begin{array}{cc}
\mathbf{R}_{n}(\mathbf{q}) & \mathbf{0}_{3 \times 3} \\
\mathbf{0}_{3 \times 3} & \mathbf{I}_{3}
\end{array}\right] \mathbf{J}_{b}(\mathbf{q})
$$

the total tip wrench $\left(\mathbf{W}_{t}\right)$ can be defined as independent of the manipulator configuration. Hence, the following reformulation of (4.12) holds:

$$
\begin{aligned}
& \mathbf{J}_{a}^{T}(\mathbf{q}) \mathbf{W}_{t}=\mathbf{C q}, \\
& \mathbf{W}_{t}=\left[\begin{array}{l}
\mathbf{F}_{g} \\
\boldsymbol{\tau}_{\mu}
\end{array}\right]=\left[\begin{array}{lllll}
\mathbf{0}_{1 \times 2} & -F_{g} & \tau_{\mu}^{x} & \tau_{\mu}^{y} & 0
\end{array}\right]^{T} .
\end{aligned}
$$

Differentiation of (4.18) establishes the following relation between the rate of change of wrench elements $\left(\dot{\mathbf{W}}_{t}=\left[\begin{array}{ll}\mathbf{0}_{1 \times 3} & \dot{\tau}_{\mu}^{T}\end{array}\right]^{T}\right)$ and the PRBM joint rate $(\dot{\mathbf{q}})$ :

$$
\mathbf{C} \dot{\mathbf{q}}=\dot{\mathbf{J}}_{a}(\mathbf{q})^{T} \mathbf{W}_{\mathbf{t}}+\mathbf{J}_{a}(\mathbf{q})^{T} \dot{\mathbf{W}}_{t}
$$

Using the chain rule, the product of $\mathbf{W}_{\mathbf{t}}$ and the rate of the Jacobian transpose $\left(\dot{\mathbf{J}}_{a}(\mathbf{q})^{T}\right)$ is represented in a form, which is linear in $\dot{\mathbf{q}}$

$$
\mathbf{C} \dot{\mathbf{q}}=\boldsymbol{\Gamma}\left(\mathbf{q}, \boldsymbol{\tau}_{\mu}\right) \dot{\mathbf{q}}+\mathbf{J}_{a}(\mathbf{q})^{T} \dot{\mathbf{W}}_{t},
$$

where

$$
\boldsymbol{\Gamma}\left(\mathbf{q}, \boldsymbol{\tau}_{\mu}\right)=\left[\begin{array}{c}
-\frac{\partial J_{a}^{3,1}}{\partial \mathbf{q}} F_{g}+\frac{\partial J_{a}^{4,1}}{\partial \mathbf{q}} \tau_{x}^{\mu}+\frac{\partial J_{a}^{5,1}}{\partial \mathbf{q}} \tau_{y}^{\mu} \\
\ldots \\
-\frac{\partial J_{a}^{3,2 n}}{\partial \mathbf{q}} F_{g}+\frac{\partial J_{a}^{4,2 n}}{\partial \mathbf{q}} \tau_{x}^{\mu}+\frac{\partial J_{a}^{5,2 n}}{\partial \mathbf{q}} \tau_{y}^{\mu}
\end{array}\right]
$$

Hence, the final equation for PRBM joint rate becomes

$$
\dot{\mathbf{q}}=\left(\mathbf{C}-\boldsymbol{\Gamma}\left(\mathbf{q}, \boldsymbol{\tau}_{\mu}\right)\right)^{-1} \mathbf{J}_{a}^{T}(\mathbf{q})\left[\begin{array}{ll}
\mathbf{0}_{1 \times 3} & \dot{\boldsymbol{\tau}}_{\mu}^{T}
\end{array}\right]^{T} .
$$


Differential equation (4.23) is used to design a MIMO inverse model controller to control the spatial position of the catheter tip $\left(\mathbf{p}_{n}\right)$. If the transformed body Jacobian $\left(\mathbf{J}_{a}(\mathbf{q})\right)$ is decomposed into linear $\left(\mathbf{J}_{a, v} \in \mathbb{R}^{3 \times 2 n}\right)$ and angular $\left(\mathbf{J}_{a, \omega} \in \mathbb{R}^{3 \times 2 n}\right)$ parts, such that $\mathbf{J}_{a}(\mathbf{q})=\left[\begin{array}{ll}\mathbf{J}_{a, v}^{T}(\mathbf{q}) & \mathbf{J}_{a, \omega}^{T}(\mathbf{q})\end{array}\right]^{T}$, the following holds:

$$
\dot{\mathbf{p}}_{n}=\mathbf{J}_{a, v}(\mathbf{q}) \dot{\mathbf{q}} \text {. }
$$

Therefore, the relation between spatial tip velocity $\left(\dot{\mathbf{p}}_{n}\right)$ and the rate of magnetic torque $\left(\dot{\boldsymbol{\tau}}_{\mu}\right)$ is as follows:

$$
\dot{\mathbf{p}}_{n}=\underbrace{\mathbf{J}_{a, v}(\mathbf{q})\left(\mathbf{C}-\boldsymbol{\Gamma}\left(\mathbf{q}, \boldsymbol{\tau}_{\mu}\right)\right)^{-1} \mathbf{J}_{a, \omega}^{T}(\mathbf{q})}_{\mathbf{A}\left(\mathbf{q}, \boldsymbol{\tau}_{\mu}\right)} \dot{\boldsymbol{\tau}}_{\mu}
$$

No torque can be generated in the direction parallel to the magnetic dipole $(\boldsymbol{\mu})$, as follows from (1.1) and (4.19). Thus, only two degrees of freedom can be controlled using the system described by (4.25), using torques $\left(\tau_{\mu}^{x}\right)$ and $\left(\tau_{\mu}^{y}\right)$. We mitigate that problem by including in the system an automated insertion device, which applies a linear displacement $(\lambda \in \mathbb{R})$ along the catheter insertion axis $(\hat{\boldsymbol{\lambda}})$, as depicted in Figure 4.2. Since this displacement can be controlled directly, we create the final actuation matrix $\left(\mathbf{A}_{s}\left(\mathbf{q}, \boldsymbol{\tau}_{\mu}\right) \in \mathbb{R}^{3 \times 3}\right)$ used for steering as follows:

$$
\mathbf{A}\left(\mathbf{q}, \boldsymbol{\tau}_{\mu}\right) \dot{\boldsymbol{\tau}}_{\mu}+\widehat{\boldsymbol{\lambda}}^{T} \dot{\lambda}=\mathbf{A}_{s}\left(\mathbf{q}, \boldsymbol{\tau}_{\mu}\right)\left[\begin{array}{ccc}
\dot{\tau}_{\mu}^{x} & \dot{\tau}_{\mu}^{y} & \dot{\lambda}
\end{array}\right]^{T}
$$

Using the matrix $\left(\mathbf{A}_{s}\left(\mathbf{q}, \boldsymbol{\tau}_{\mu}\right)\right)$, the inverse-model controller can be formulated. We define the position error vector $\left(\mathbf{e} \in \mathbb{R}^{3}\right)$ such that

$$
\mathbf{e}=\mathbf{p}_{n}^{r e f}-\mathbf{p}_{n}^{e s t}
$$

where $\mathbf{p}_{n}^{r e f} \in \mathbb{R}^{3}$ is the reference tip position and $\mathbf{p}_{n}^{e s t} \in \mathbb{R}^{3}$ is the current position estimate coming from the shape sensing technique described in Section 4.3. Using the quasi-static approach, the control equation takes the form

$$
\mathbf{A}_{s}(\mathbf{q})^{-1}\left(\dot{\mathbf{p}}_{n}^{r e f}+\mathbf{K e}\right)=\left[\begin{array}{lll}
\dot{\tau}_{\mu}^{x} & \dot{\tau}_{\mu}^{y} & \dot{\lambda}
\end{array}\right]^{T}
$$

where $\mathbf{K} \in \mathbb{R}^{3 \times 3}$ is a diagonal, positive-definite gain matrix, and $\dot{\mathbf{p}}_{n}^{\text {ref }}$ is the feed-forwarded reference tip velocity. The magnetic control torques are computed by numerically integrating the expressions $\left(\dot{\tau}_{\mu}^{x}\right.$ and $\left.\dot{\tau}_{\mu}^{y}\right)$ given in (4.28). The corresponding reference magnetic field $\left(\check{\mathbf{B}}_{\mathcal{G}} \in \mathbb{R}^{3}\right)$ is consequently calculated using the following inverse map

$$
\check{\mathbf{B}}_{\mathcal{G}}=\left\|\boldsymbol{\mu}_{e s t}\right\|^{-2} S\left(\boldsymbol{\mu}_{e s t} \mathbf{R}_{n}(\mathbf{q}) \boldsymbol{\tau}_{\mu} .\right.
$$

In our study, $\check{\mathbf{B}}_{\mathcal{G}}$ is generated by an array of mobile electromagnetic coils using the technique presented in Chapter 2. 


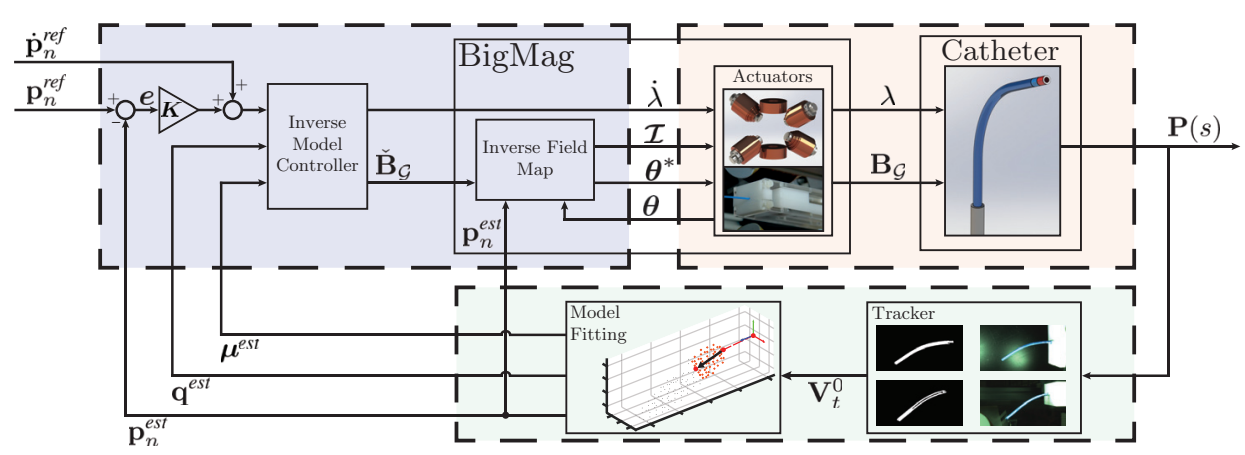

Figure 4.4: The block diagram summarizing the system for closed-loop steering of magnetic catheters. An inverse model control (blue) is used to track a pre-programmed position trajectory $\left(\mathbf{p}_{n}^{r e f}\right)$ of the tip of magnetic catheter using an inverse model control based on PRBM of the catheter. The controller provides reference magnetic field $\left(\check{\mathbf{B}}_{\mathcal{G}}\right)$ and catheter insertion velocity $(\dot{\lambda})$, which minimize the steering error $(\mathbf{e}) . \dot{\lambda}$ is a control variable fed directly to the automated catheter inserter. In the same time $\check{\mathbf{B}}_{\mathcal{G}}$ is generated using BigMag, using the technique presented in Chapter 2 to provide the rest of control variables: coil currents $(\mathcal{I})$ and reference fixture positions $(\check{\boldsymbol{\theta}})$, generating actual magnetic field $\left(\mathbf{B}_{\mathcal{G}}\right)$. This field determines the shape of the catheter $(\mathbf{P}(s))$. The shape-sensing (green) is provided for feedback in form of estimated tip position $\left(\mathbf{p}_{n}^{e s t}\right)$, tip magnet dipole value $\left(\boldsymbol{\mu}^{e s t}\right)$ and pseudo-rigid-body configuration $\left(\mathbf{q}^{\text {est }}\right)$. These variables are recovered from a point cloud $\left(\mathbb{V}_{t}^{0}\right)$ containing the silhouette of the catheter segmented from a tracker based on stereo vision.

\subsection{Experimental Validation}

The control system (Figure 4.4) comprising of elements proposed in Sections 4.2-4.4 is implemented in $\mathrm{C}++14$ on a computer running Linux Ubuntu 14.04.01, equipped with Intel Xeon E5 CPU, NVidia Quadro K4200 GPU and 32 GB RAM. First, we test the shape sensing technique presented in Section 4.3. The shape sensing is implemented within BigMag to work with real-time point cloud data provided by a stereo vision system. Finally, a complete closed-loop steering framework is used to trace a series of $3 \mathrm{D}$ trajectories within the workspace of BigMag.

\subsubsection{Shape Sensing Validation}

The shape sensing method, presented in Section 4.3 is implemented within BigMag system to process point cloud generated using the shape-from-silhouette approach [202]. The silhouette of the catheter is registered using stereo images coming from two Dalsa Genie Nano C1940 Red-Green-Blue (RGB) cameras (Teledyne Dalsa, Waterloo, Canada) (Figure 2.2). The side camera is equipped with Cinegon 1.6/16 (Schneider, Rueil-Malmaison, France) lens providing a depth of field of $120 \mathrm{~mm}$ with near limit of $111.2 \mathrm{~mm}$. The top camera employs Cinegon 10/1.9 (Schneider, Rueil-Malmaison, France) lens, providing an infinite field depth with near limit of $52.1 \mathrm{~mm}$. Both cameras are calibrated in a stereo configuration using Camera Calibration Toolbox (Mathworks, Natick, USA). Segmented silhouettes are used to generate catheter point cloud using space carving technique. The resulting point cloud $\left(\mathbb{V}_{t}^{0}\right)$ is fed into Algorithm 1 for shape sensing. 
The validation of the shape sensing involves using the technique presented in Section 4.3 on a catheter mock-up with predefined curvature (Figure 4.5). This mock-up is designed using CAD technique and fabricated on a high resolution $3 \mathrm{D}$ printer. The known shape $(\mathbf{P}(s))$ for a set of points along the length of the mock-up is compared against the shape estimate function $(\widetilde{\mathbf{P}}(s))$ using Algorithm 1 with three different polynomial orders $(\zeta=4,5,6)$. The error between $\mathbf{P}(s)$ and $\widetilde{\mathbf{P}}$ was measured in a total of 200 locations $(s)$. The average errors are $1.5 \mathrm{~mm}$ for $\zeta=4,0.88 \mathrm{~mm}$ for $\zeta=5$, and $0.59 \mathrm{~mm}$ for $\zeta=6$.
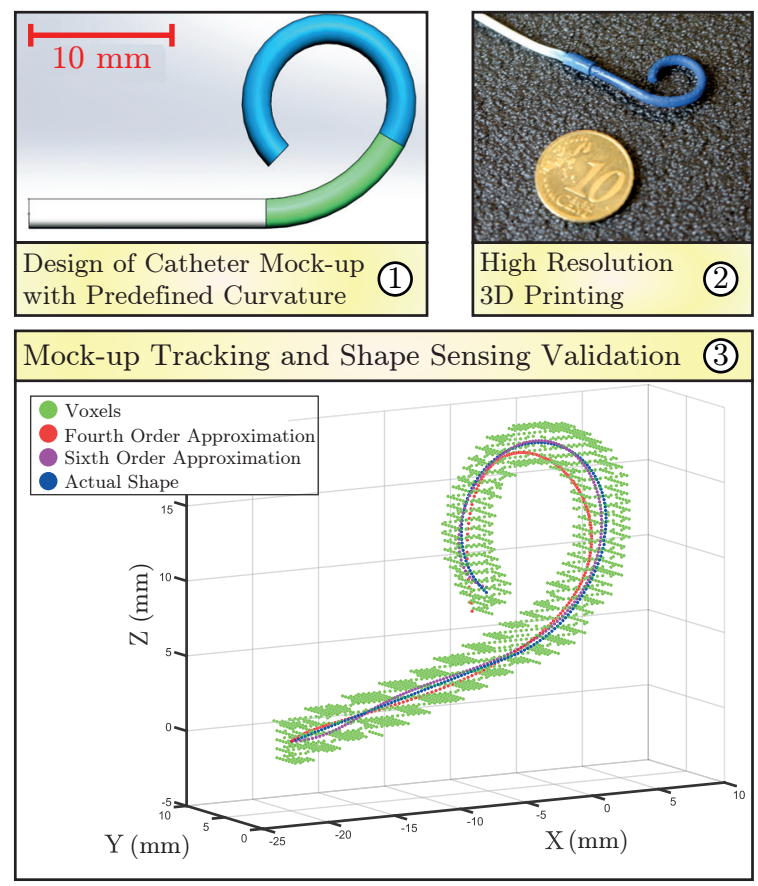

Figure 4.5: The experimental procedure used to validate the shape sensing technique. A mock-up of a catheter with predefined shape is designed (1), and fabricated using 3D printing technique (2). The shape sensing technique proposed in this chapter is used to retrieve polynomial shape estimate functions of different order (3). Those estimates are compared against known mock-up shape. The best results were observed for sixth order polynomial, with mean error of $0.59 \mathrm{~mm}$.

\subsubsection{Closed-Loop Steering}

Ultimately, the system proposed in this chapter is tested in final steering experiments. First, the implementation of the control framework has been benchmarked for computational performance. The average execution time of different software components is as follows: BigMag field map (Chapter 2, Section 2.3) - $350 \mu \mathrm{s}$; inverse model controller (Sections 4.2 and 4.4) - $1.8 \mathrm{~ms}$; stereo image acquisition and processing - $35.2 \mathrm{~ms}$, shape reconstruction (Section 4.4) - $2.4 \mathrm{~ms}$. The total computational time of software components allows for $25 \mathrm{~Hz}$ closed loop control. Nevertheless, to eradicate all possible jitter, predominantly due to variable image 
acquisition time (up to $60 \mathrm{~ms}$ ), the actual control cycle is set to $10 \mathrm{~Hz}$.

The controller is used to track three trajectories presented in the Figure 4.6, each with different speed. The results show that the proposed control framework can be used for position steering of catheter tip with minimum recorded mean position trajectory error of $0.57 \mathrm{~mm}$ (RMS error $0.77 \mathrm{~mm}$ ). The maximum reached catheter speed was $5 \mathrm{~mm} / \mathrm{s}$.

The steering errors can be explained by several phenomena observed during the experiments. First of all, the vision-based shape sensing method generates noise, mostly due to limitations of the catheter base detection scheme (Algorithm 1, Lines 1-2). This noise can be observed in Figure 4.6 as a fast-oscillating component of the error signal. Furthermore, the reduced order of the system due to quasi-static assumption caused further performance deterioration at higher velocities, due to the uncompensated higher-order dynamics of the catheter, as well as relatively low frequency of the control cycle. Moreover, at larger tip deflection the PRB modelling approach becomes less accurate, degrading the performance of the controller. Finally, the effect of magnetic force $\left(\mathbf{F}_{\mu}\right)$ resulted in larger steering error close to the edges of BigMag workspace, where magnetic field gradients are more prominent.

The maximum reported catheter position error of $6.0 \mathrm{~mm}$ does not guarantee reliable operation within smaller sections of human cardiovascular system. Even though high compliance of the device, as well as small magnitudes of control torques prevent significant tissue damage, the positioning precision should be improved. The decrease of the maximum error at lower velocities of the catheter indicates that increasing the bandwidth of the control system, or extending the controller to account for the dynamics of both the catheter and the coil system, could contribute to that.

Improving several other aspects of the presented framework can facilitate the translation of magnetic catheter steering into clinical practice. The current size of workspace offered by BigMag restricts the procedures to catheter positioning tasks within human limbs. Development of a system capable of running the presented framework at sufficient bandwidth in a clinically-relevant workspace remains a relevant, ongoing technical challenge.

Furthermore, the experiments performed in this chapter demonstrate the performance of our catheter in a highly controlled environment. The motion of the catheter occurs in air. Such conditions cannot be deemed realistic, as inside the body cavities the catheter is likely to be surrounded by physiological fluids, such as blood, urine or the contents of the gastrointestinal tract. In such circumstances, the drag force acting on the catheter is likely to play significant role in the dynamical behaviour of the device. On one hand, this may have a beneficial effect on the performance of the catheter, as increased damping is likely to minimize the high-frequency vibrations. However, the presence of fluid moving at significant velocities, such as blood in beating heart, is expected to be a significant source of disturbance for the controller, possibly further exacerbated by the biological motion of the surrounding soft tissue. Therefore, our controller should be expanded to involve strategies for motion compensation, in order to enable its successful operation in vivo $[32,70]$.

Finally, the majority of clinical procedures require achieving successful, pre- 


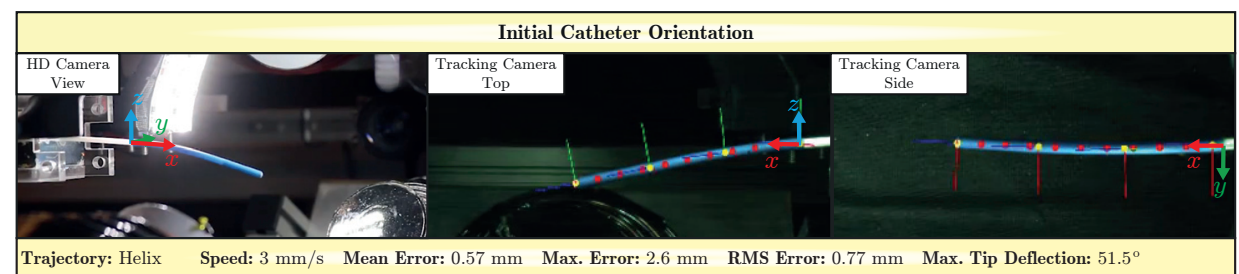

Trajectory: Helix Speed: $3 \mathrm{~mm} / \mathrm{s} \quad$ Mean Error: $0.57 \mathrm{~mm} \quad$ Max. Error: $2.6 \mathrm{~mm}$ RMS Error: $0.77 \mathrm{~mm}$ Max. Tip Deflection: $51.5^{\circ}$
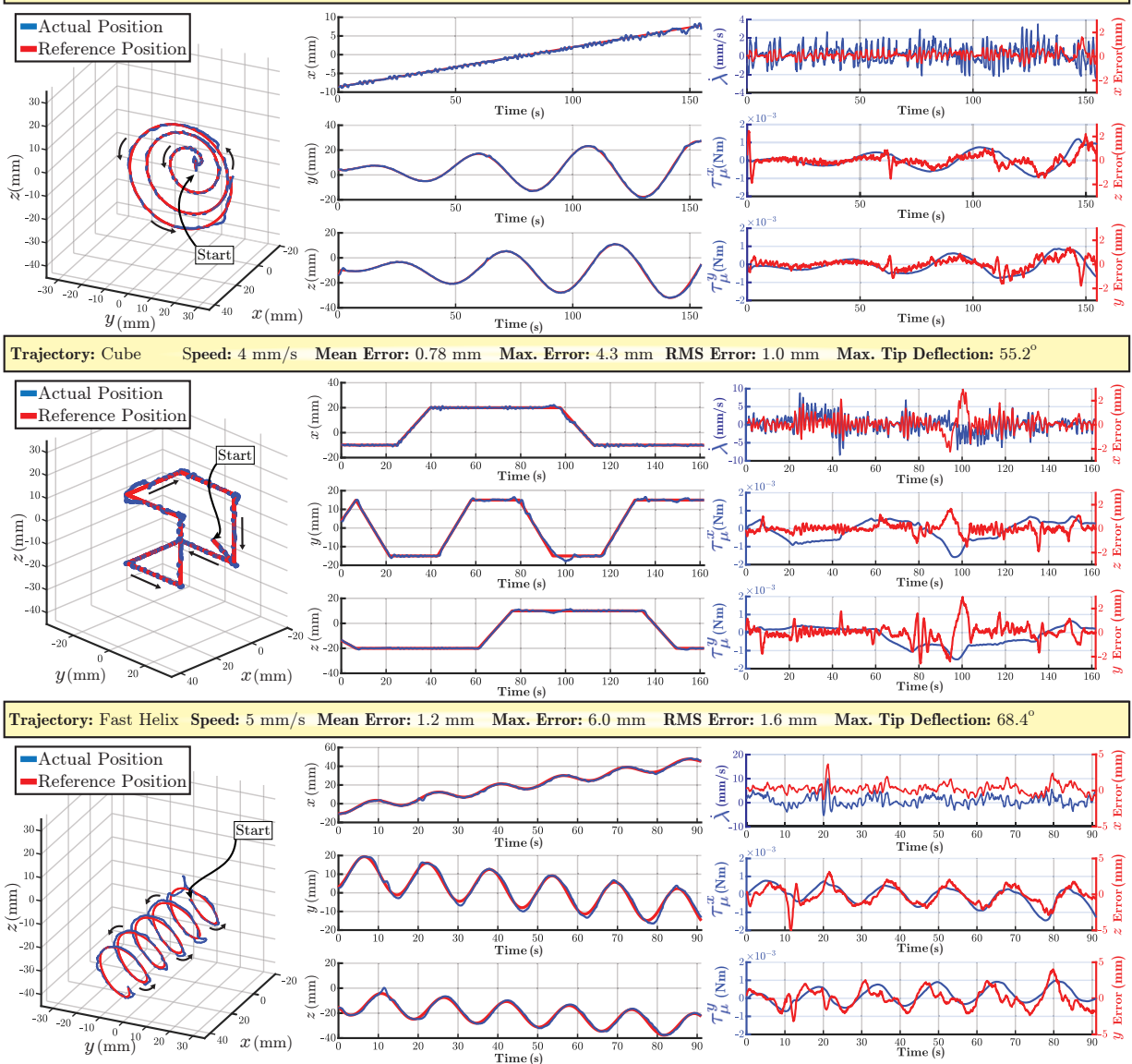

Figure 4.6: The catheter is steered inside the workspace $\left(\boldsymbol{\Omega}_{w}\right)$ of BigMag along three trajectories with various geometries and speeds. For each trajectory the mean, maximum and RMS steering error is measured. Left: The spatial trajectory of catheter tip in carthesian coordinates $(x, y, z)$ of $\boldsymbol{\Omega}_{w}$. Centre: Time plot of reference and actual tip position for each spatial coordinate Right: Time plot of error signal (e) spatial components, and outputs of control equation (4.28): magnetic torques $\left(\tau_{\mu}^{x}\right)$ and $\left(\tau_{\mu}^{y}\right)$, and catheter insertion velocity $(\dot{\lambda})$.

dictable contact between the catheter and the tissues of the body. This limits the applicability of the framework, as it does not include the presence of contact forces. Including these forces in the PRB model, and developing a reliable force sensing method could offer the possibility of realising force control in a manner similar to [196]. 


\subsection{Conclusions}

In this chapter a modular approach for closed-loop control of magnetic endovascular catheters is presented. The catheter is represented using pseudo-rigid-body modelling, taking into account the effects of magnetic and gravitational forces. Based on PRBM approach, a non-linear, inverse-model position controller is derived for steering catheter using magnetic actuation. The magnetic field used for steering is generated by BigMag, an array of mobile electromagnets. A stereovision system is used to provide catheter shape information, as well as to track the catheter tip position to close the control loop.

The presented framework is tested experimentally. The shape sensing technique is validated as capable of retrieving the shape of the catheter with a mean error of $0.59 \mathrm{~mm}$. Finally, all the components of the system are integrated and the framework is evaluated in catheter steering experiments across a set of trajectories. The minimum reported mean position error is $0.57 \mathrm{~mm}$ (RMS error $0.77 \mathrm{~mm}$ ), and the maximum catheter tip speed achieved is $5 \mathrm{~mm} / \mathrm{s}$. 



\section{Chapter 5}

\section{Grasping Using Magnetically-Actuated Tentacle Catheter: A Proof-Of-Concept Study}

\section{Abstract}

The use of magnetically-actuated catheters has the potential to facilitate minimally-invasive surgical procedures. In this chapter we present a magneticallyactuated tentacle catheter for object manipulation in remote areas of the human body. The catheter employs a bio-inspired technique of underactuated grasping. The whole body of the catheter loops around the target object, providing form closure necessary for manipulation. The catheter employs a permanent magnet to steer the position of its tip, and an electromagnetic coil to strengthen the maximum force applicable to the target object. We test the catheter in a series of proof-of-concept experiments. In a thermal study, we show that within the operational conditions, the heat dissipated by the coil allows for safe operation of tentacle catheter within the human body. Subsequently, we characterise the maximum force available for manipulation using a force sensor. The tentacle catheter can apply forces up to $0.1 \mathrm{~N}$, which is in accordance with finite-element simulation. Finally, we demonstrate the operation of the tentacle catheter in a task involving manipulation of porcine tissue.

Adapted from:

J. Sikorski, E.S.A.A.M. Rutting, S. Misra. Grasping using magneticallyactuated tentacle catheter: a proof-of-concept study, Proceedings of the 7th IEEE RAS/EMBS International Conference on Biomedical Robotics and Biomechatronics (BIOROB 2018), Enschede, The Netherlands, pp. 609-614, August 2018 


\subsection{Introduction}

In the last few years, research in continuum robotics has developed new classes of tools for minimally-invasive surgical (MIS) procedures [41]. Among these tools, automated catheters exhibit potential of becoming versatile robotic manipulators, capable of performing various surgical tasks inside the human body. Standard, manually-operated catheters are mostly used for a small number of MIS procedures within endovascular surgery. The applications of standard catheters are limited mostly due to lack of precision, resulting in time-consuming navigation, which is challenging even for experienced clinicians. By employing automated steering techniques, the precision of catheters can be improved, making them easier to operate. This would not only improve the outcome of current procedures, but also extend the number of MIS procedures which can be performed using catheters [65].

The versatility of catheters comes from their favourable mechanical properties. They comprise of sleek, soft polymeric tubes with inherent compliance, which facilitates safe operation within the human body. Small size of the catheters enables them to access difficult locations with minimal trauma. Steering of larger catheters is usually performed using tendons [81]. However, friction in tendon-driven instruments leads to safety concerns and limits their scalability [172]. Furthermore, the tendons hinder compliance matching between the catheter and the human tissue, decreasing the benefits of using flexible catheters [39].

Magnetic actuation has been investigated as a viable solution to these problems [134]. Several studies have already demonstrated steering of magneticallyactuated catheters. Nevertheless, the principal emphasis therein is placed on establishing theoretical foundation of catheter mechanics and control $[116,117,144,203]$. Despite being a remarkable milestone, these studies seek to improve the performance of catheters for standard MIS procedures. A few other studies suggested application of magnetic catheters for eye surgery, but their focus does not go beyond the standard task involving developing a point contact between the catheter tip and the tissue $[95,174]$.

Current literature certainly does not exhaust the opportunities offered by magnetic catheters. One of the tasks, which in the future can be realised by catheters is grasping, i.e., achieving a stable physical contact and force transmission between the manipulator and target object. It is required for object manipulation in various MIS procedures such as biopsies, keyhole surgery and targeted drug delivery. The grasping tasks in those procedures are usually realised by mechanically-complex jaw-like forceps [204]. These forceps are hard to miniaturize and actuate remotely, therefore their dexterity within the human body is limited [205].

In this chapter we design and test a magnetically-actuated tentacle catheter for grasping tasks inside the human body (Figure 5.1). This catheter employs a bioinspired underactuated grasping technique. This technique has been demonstrated in larger, industrial continuum manipulators, but has not yet been applied for medical procedures [206]. Due to a favourable distribution of the contact force, continuum manipulators employing underactuated grasping are considered safer for object manipulation than classical forceps [39]. Moreover, their simple design allows for successful miniaturization and use in MIS procedures.

The tentacle catheter presented in this study employs a permanent magnet 


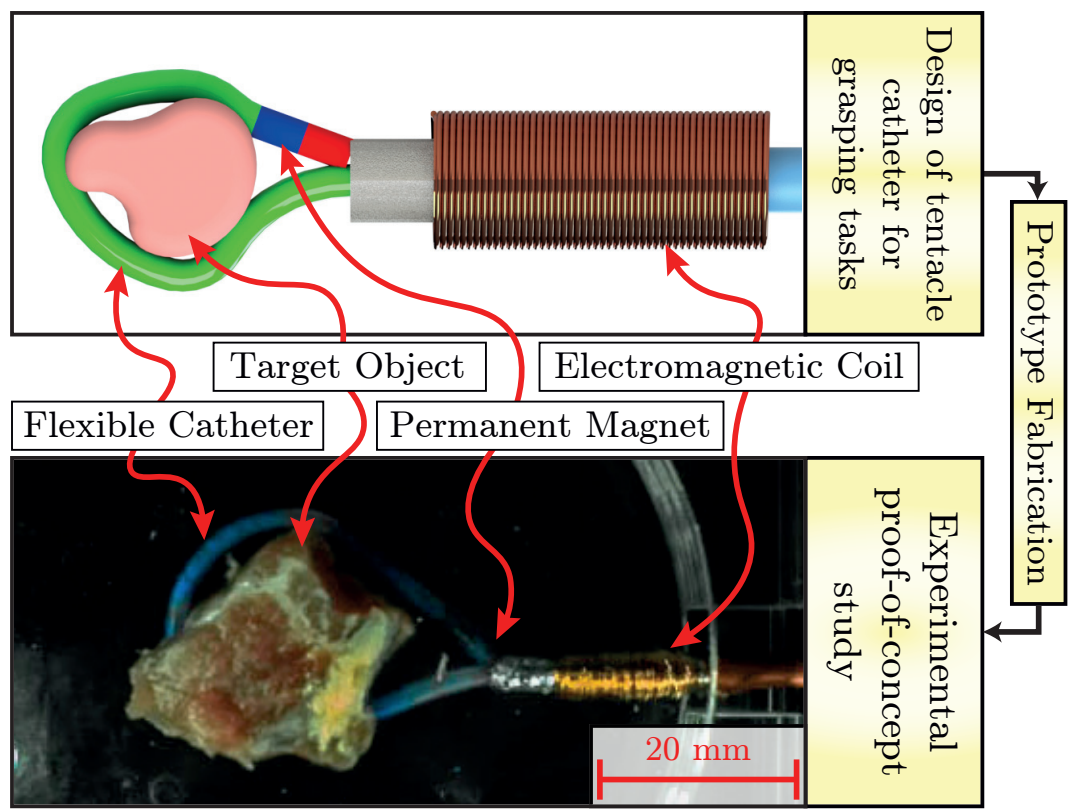

Figure 5.1: In this study we propose a catheter for execution of grasping tasks for minimallyinvasive surgical procedures. The catheter employs an underactuated grasping technique, looping its whole body around the target object. For that purpose, the catheter is steered using magnetic actuation enabled by a permanent magnet at its tip. Furthermore, an electromagnetic coil incorporated into the body of the catheter is used to strengthen the grasp, allowing for reliable object manipulation. This concept, bio-inspired by tentacle grasping has been selected during the design phase, due to suitability for operation within human body, as well as for the compatibility with pre-existing magnetic systems. A prototype of the tentacle catheter has been fabricated and tested during the series of proof-of-concept experiments. Among other tests, we demonstrate that the tentacle catheter is capable of grasping and manipulating porcine tissue during a pickand-place tasks.

at its flexible tip. The primary function of the magnet involves steering of the catheter into the desired location and looping it around a target object, using the interaction with external magnetic fields. However, due to high compliance of the catheter, the force required for manipulation of the object deforms the catheter tip, preventing successful grasps. As a remedy to that problem, we incorporate an electromagnetic coil into the body of the catheter. With the tip of the catheter looped around the target object, the coil is used to exert a large attraction force on the permanent magnet. This force strengthens the grasp and allows for object manipulation. We test our design experimentally, using BigMag (presented in Chapter 2) for magnetic actuation. We employ a force sensor to quantify the strength of the grasp and compare the results with a finite element simulation. Finally, we demonstrate the tentacle catheter performing a simple pick-and-place task using biological material.

The rest of this chapter is structured as follows. Section 5.2 introduces and analyses three proposed conceptual designs for grasping catheters, and presents the details of the tentacle catheter employing underactuated grasping. Section 5.3 shows the results of a proof-of-concept study demonstrating the feasibility of the tentacle catheter. Finally, Section 5.4 summarises the chapter, giving recommendations for further study. 


\subsection{Catheter as a Grasping Tool - Conceptual De- sign}

To authors best knowledge, the idea of realising grasping tasks within human body using (magnetically-actuated) catheters has not yet been discussed in the literature. This necessitates starting the investigation with a design process, in which possible grasping strategies are generated and translated into novel concepts of catheters. This section describes that process, proposing and analysing three concepts of grasping catheters. The concepts are assessed using a set of design requirements. Based on these requirements, we show that the catheter employing underactuated grasping shows distinct advantages in terms of its versatility, applicability and miniaturization capabilities. Thus, in this section we give the details of the proposed underactuated grasping strategy, chosen to be verified further in the chapter with a set of proof-of-concept studies.

\subsubsection{Design Requirements and Preliminary Concepts}

This study aims at development of a magnetically-actuated catheter with a potential of performing grasping tasks within remote locations inside the human body. We begin by establishing a set of requirements for such a device (Table 5.1). Using those requirements, three concepts for magnetically-actuated grasping catheters are generated during the design phase (Figure 5.2). The concepts are analysed and assessed for their conformity with the design requirements.

After careful analysis of the design requirements, the forceps are considered unsuitable for the target application for a number of reasons. The complex mechanical structure of the forceps is difficult to manufacture using rapid-prototyping techniques. Since a breaking feature is used, the forceps are not reusable. After a grasping attempt, the catheter loses its functionality and needs to be extracted from the body. Furthermore, the resulting end-effector is considered too stiff to allow for safe operation within the body.

The first concept aims at miniaturizing the standard, robotic forceps using magnetic actuation (Figure 5.2.(a). The forceps are located at the tip of the catheter in a hollow, rigid tube extending from the flexible body of the catheter. A permanent magnet located at the end of the tube is used both in catheter steering and grasping. The forceps are 3D printed as a single part, held open by a small portion of material called breaking feature. The forceps are fitted with electromagnetic coil. When delivered to the location of the target object, the electromagnetic coil is activated. This creates a magnetic attraction force $\left(\mathbf{F}_{a} \in \mathbb{R}^{3}\right)$ pulling the forceps towards the magnet. This force destroys the breaking feature, allowing the forceps to grasp the target object.

In an attempt to address those challenges a second concept is devised, in which the forceps are substituted with a hook (Figure 5.2.(b)). Having a much simpler mechanical structure, a hook offers fewer failure scenarios, and can be successfully fabricated at different scales. Albeit appealing, this concept revealed another set of problems inherent to grasping using catheters. For successful grasp, both the forceps and the hook need to approach the target object at a particular orientation. This is enabled only if the manipulator allows for $6 \mathrm{DoF}$ control of the end-effector 
Table 5.1: Design Requirements for Grasping Catheter

\begin{tabular}{|c|c|c|c|c|}
\hline \multirow[b]{2}{*}{ Requirement } & \multirow[b]{2}{*}{ Motivation } & \multicolumn{3}{|c|}{ Catheter Concepts } \\
\hline & & $\begin{array}{l}\text { Magnetic } \\
\text { Forceps }\end{array}$ & $\begin{array}{c}\text { Steerable } \\
\text { Hook }\end{array}$ & $\begin{array}{l}\text { Tentacle } \\
\text { Catheter }\end{array}$ \\
\hline $\begin{array}{l}\text { The catheter is } \\
\text { capable of grasping } \\
\text { objects within } \\
\text { human body. }\end{array}$ & $\begin{array}{l}\text { Primary function } \\
\text { of the catheter. } \\
\text { Therefore, also the } \\
\text { main design } \\
\text { requirement. }\end{array}$ & $\checkmark$ & $\checkmark$ & $\checkmark$ \\
\hline $\begin{array}{l}\text { The catheter has } \\
\text { sleek shape and is } \\
\text { primarily made of } \\
\text { flexible materials. }\end{array}$ & $\begin{array}{l}\text { Improves safety } \\
\text { and dexterity of } \\
\text { the device inside } \\
\text { the cavities of } \\
\text { human body. }\end{array}$ & $x$ & $\checkmark$ & $\checkmark$ \\
\hline $\begin{array}{l}\text { The catheter has a } \\
\text { simple and } \\
\text { robust structure. }\end{array}$ & $\begin{array}{l}\text { Limits the number } \\
\text { of possible failure } \\
\text { scenarios, } \\
\text { contributing to } \\
\text { safety of the } \\
\text { patient. }\end{array}$ & $x$ & $\checkmark$ & $\checkmark$ \\
\hline $\begin{array}{l}\text { The concept has a } \\
\text { potential for } \\
\text { miniaturization } \\
\text { using currently } \\
\text { available fabrication } \\
\text { techniques. }\end{array}$ & $\begin{array}{l}\text { Increases the } \\
\text { number of } \\
\text { potential } \\
\text { applications within } \\
\text { minimially-invasive } \\
\text { surgery. }\end{array}$ & $x$ & $\sqrt{ }$ & $\checkmark$ \\
\hline $\begin{array}{l}\text { The catheter is } \\
\text { reusable after } \\
\text { performing a } \\
\text { grasping task. }\end{array}$ & $\begin{array}{l}\text { Allows for } \\
\text { manipulation of } \\
\text { multiple objects } \\
\text { during a single } \\
\text { procedure. }\end{array}$ & $x$ & $x$ & $\sqrt{ }$ \\
\hline $\begin{array}{l}\text { The grasping } \\
\text { technique is } \\
\text { compatible with } \\
\text { pre-existing devices } \\
\text { for magnetic } \\
\text { manipulation. }\end{array}$ & $\begin{array}{l}\text { Ensures the } \\
\text { concept is realistic } \\
\text { and can be realised } \\
\text { within the current } \\
\text { state-of-the-art } \\
\text { technology. }\end{array}$ & $x$ & $x$ & $\checkmark$ \\
\hline
\end{tabular}

pose. This is challenging to realise due to limitations of magnetic actuation, and has not yet been demonstrated in the literature [144].

Ultimately, we propose the catheter realising the underactuated grasping technique [206]. This technique is inspired by biological structures like vines, tentacles or elephant trunks. The device is sleek and compliant; its structure is simple and shows potential for miniaturization. The underactuated grasping technique is versatile, allowing the device to grasp targets of different sizes and shapes. Furthermore, the grasping using this technique does not require precise pose control of catheter tip. Thus, the tentacle catheter is compatible with the existing systems for magnetic actuation. Therefore this design fulfils all the requirements presented in Table 5.1. 


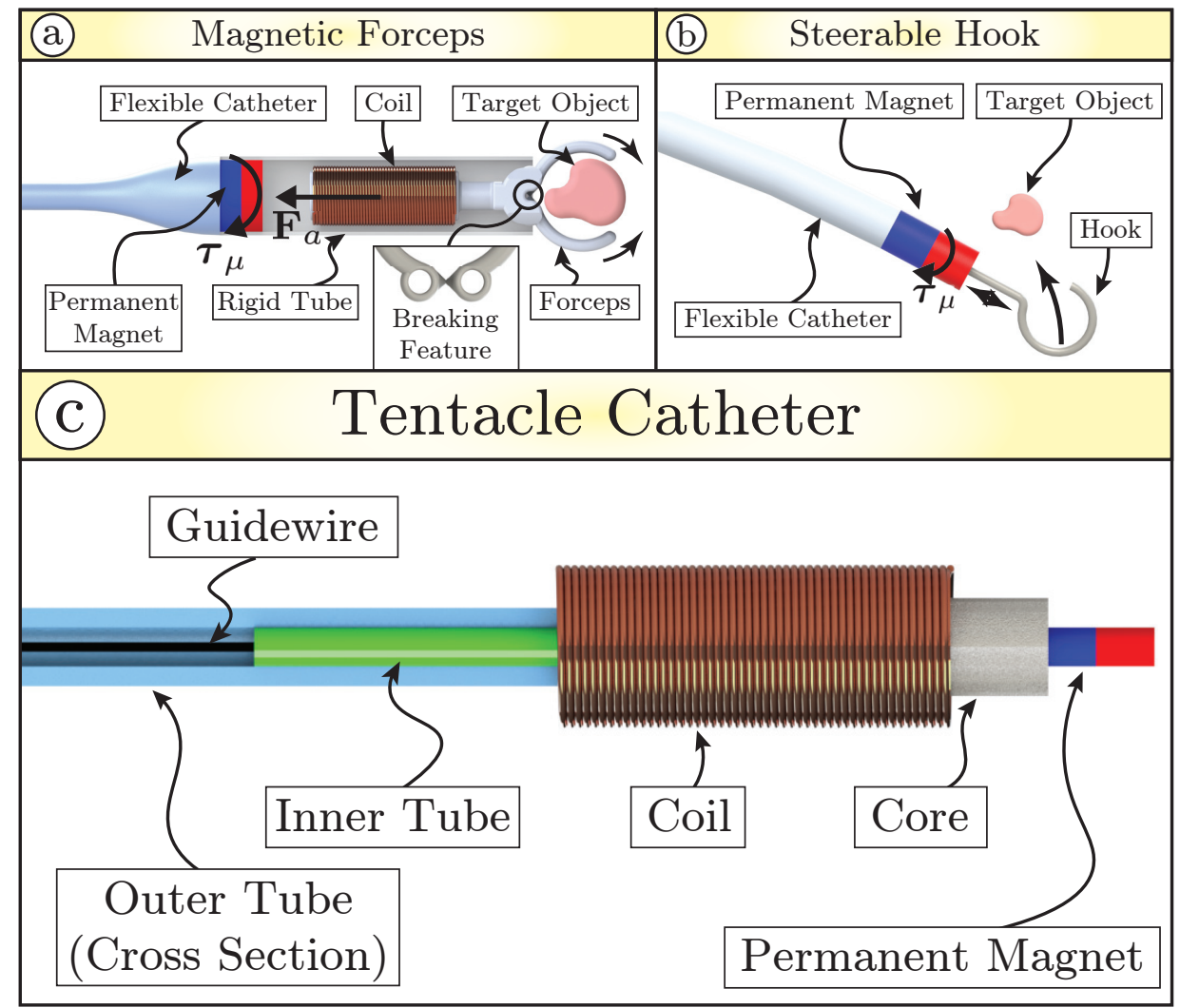

Figure 5.2: The results of conceptual design study on grasping catheters. The final solution is chosen from three generated concepts. The two initial concepts involve magnetically actuated forceps (a) and hook (b). The final design (c) addresses the limitations of the discarded concepts. It employs a catheter comprising of two concentric tubes. The inner tube has a permanent magnet at its tip. The grasping (Figure 5.3) is realised by ejecting the inner tube using a guidewire, and steering it using magnetic torque $\left(\boldsymbol{\tau}_{\mu}\right)$ to wrap around a target object. The grasping is strengthened by using an electromagnetic coil attached to the outer tube. Running the current inside the coil results in generation of large magnetic attraction force $\left(\mathbf{F}_{a}\right)$, attracting the tip magnet.

\subsubsection{Details of Tentacle Catheter}

The proposed tentacle catheter (Figure 5.2.(C) comprises of two concentric, compliant tubes. The elastic modulus of the outer tube is comparable to standard medical catheter (300 MPa), whereas the modulus of the inner tube is an order of magnitude lower ( $50 \mathrm{MPa})$. The outer tube spans the entire length of the device, from the point of insertion to the tip of the catheter. The inner tube is short, and at its proximal end it connects to a guidewire used to control its position inside the outer tube.

The grasping strategy is employed in tentacle catheter is presented in Figure 5.3. A permanent magnet, represented by its magnetic dipole moment $\left(\boldsymbol{\mu} \in \mathbb{R}^{3}\right)$, is attached at the tip of the inner tube. Exposed to external magnetic field $\left(\mathbf{B} \in \mathbb{R}^{3}\right)$, the catheter experiences a wrench $\left(\mathbf{W} \in \mathbb{R}^{6}\right)$ comprising of 


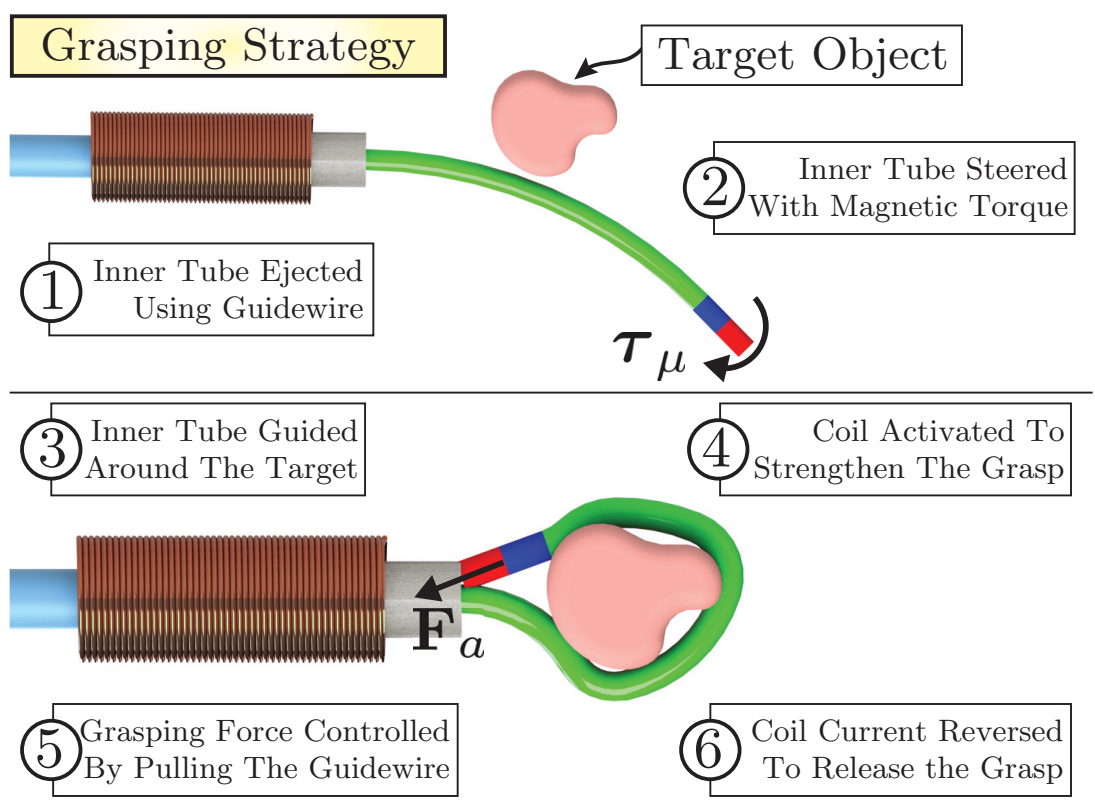

Figure 5.3: The steps (1) to (6) present the proposed grasping strategy. The catheter is navigated towards the target site using magnetic actuation. The inner tube of the catheter is ejected and steered around a target object, using magnetic torque $\left(\tau_{\mu}\right)$. The electromagnetic coil located on the outer tube is activated to attract the tip magnet using the force $\left(\mathbf{F}_{a}\right)$ forming a stable loop around the target object. By pulling the guidewire, the loop is tightened, grasping the target object. The magnitude of $\mathbf{F}_{a}$ determines the maximum possible grasping force.

magnetic force $\left(\mathbf{F}_{\mu} \in \mathbb{R}^{3}\right)$ and magnetic torque $\left(\boldsymbol{\tau}_{\mu} \in \mathbb{R}^{3}\right)$ as described by (1.1)

In a tentacle catheter, this wrench has two main applications, depending on the phase of the task. During the navigation through the body to the target site, the inner tube is fully retracted. Hence, the wrench $(\mathbf{W})$ is transmitted directly to the outer tube. This allows for the navigation of the catheter to the desired pose within the target site in a manner similar to [116]. When the catheter reaches the target site, the inner tube is extended using the guidewire. The wrench $(\mathbf{W})$ is then used to bend the inner tube around the target object, realising the underactuated grasp. Since the compliance of the inner tube is much lower than the outer one, this bending can be realised with much lower magnitude of $\mathbf{W}$.

Once the catheter is wrapped around the target object, the contact between the object and the body of the manipulator provides planar form closure [206]. However, due to the high compliance of the inner tube, the wrench $(\mathbf{W})$ itself may not be sufficient to maintain a stable grasp, especially if the manipulation of the object requires overcoming external forces. Thus, we modify the standard underactuated grasping strategy to alleviate that problem. We locate an electromagnetic coil at the end of the outer tube. Once the inner tube of the catheter is bent around the target object, the coil is activated to attract the tip magnet with a magnetic force $\left(\mathbf{F}_{a} \in \mathbb{R}^{3}\right)$, forming a loop around the target object. This loop can be tightened using the guidewire to form a stable grasp. This grasp is strengthened by $\mathbf{F}_{a}$, which therefore determines the maximum force, which can be 


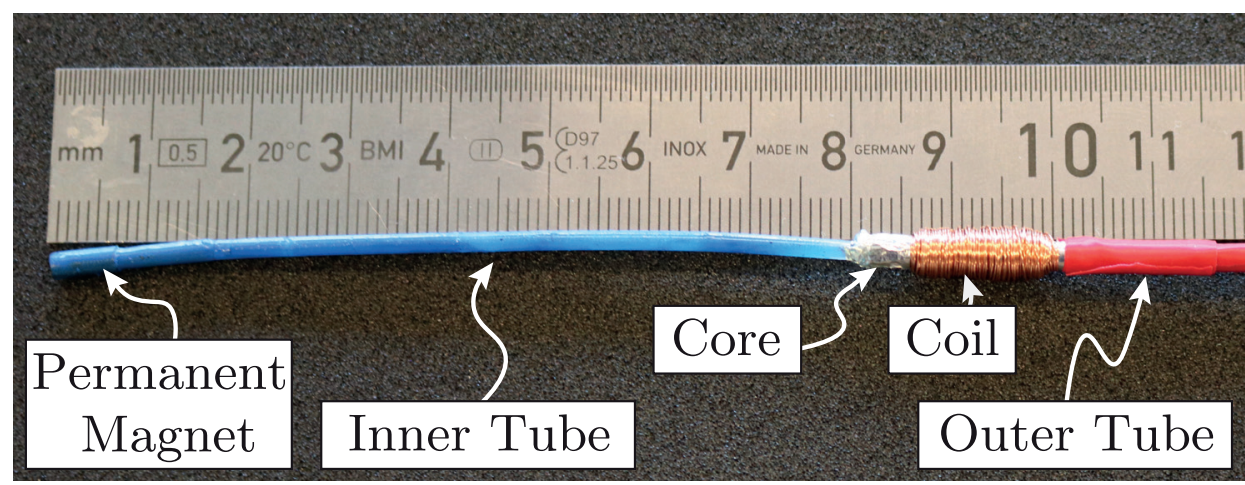

Figure 5.4: The prototype of the tentacle catheter used in the proof-of-concept study. The inner tube is made of Pellethane (Lubrizol, Wickliffe, USA) and has a fixed length of $8 \mathrm{~m}$ and a diameter of $1.5 \mathrm{~mm}$. The outer tube is made of Pebax 6333 (Arkema, Paris, France) and has a diameter of $2 \mathrm{~mm}$. The electromagnetic coil has 530 windings of $0.2 \mathrm{~mm}$ copper wire. The length of the coil is $13 \mathrm{~mm}$ and its outer diameter is $5.6 \mathrm{~mm}$. It is wound around a soft iron core with outer diameter of $2 \mathrm{~mm}$, inner diameter of $1.5 \mathrm{~mm}$ and a length of $15 \mathrm{~mm}$.

transmitted to the target object during the manipulation task.

\subsection{Tentacle Catheter - Experimental Proof-of- Concept}

After selecting the tentacle catheter as the final concept for grasping tasks, we fabricate it and demonstrate its capabilities in a series of proof-of-concept studies. The prototype of the catheter used for that purpose is presented in Figure 5.4. The mechanism for ejecting/retracting the inner tube using a guidewire (Figure 5.3, steps (1) and (5)) is not realised in our prototype. The inner tube is kept ejected at a constant length of $8 \mathrm{~mm}$. Such a catheter can successfully loop around objects with circumferences smaller than this value. The resulting grasp does not fully constrain the relative motion between the target and the manipulator body. Nevertheless, the resulting form closure allows for planar manipulations, and thus it is considered sufficient for the proof-of-concept experiments.

Three studies have been performed to test the suitability of tentacle catheter for grasping tasks. Since the heat dissipated due to resistance of the electromagnetic coil can damage the tissues of human body, we perform a thermal study to demonstrate the safety of the catheter within the desired range of currents. Consequently we test the tentacle catheter in two experiments. We characterise the maximum strength of the grasp experimentally using a force sensor, and compare it with a finite element (FE) simulation. Finally, we demonstrate that the tentacle catheter can be used for object manipulation by performing grasping task using porcine tissue.

\subsubsection{Thermal Safety Study}

The grasping technique proposed for tentacle catheter involves using an electromagnetic coil to increase the maximum manipulation force. The coil is operated 
using small direct currents (maximum of $1.25 \mathrm{~A}$ at a voltage of $5.3 \mathrm{~V}$ ), which pose no threat to human tissues. However, due to resistance of the windings, the coil dissipates substantial amount of heat. In contrast to the current, this heat can be damaging to the tissues surrounding the catheter. Therefore, demonstrating the thermal safety of the tentacle catheter is an important part of the proof-of-concept study.

The experimental setup used for studying thermal safety is presented in Figure 5.5. The prototype of the tentacle catheter is located in the tank of water kept at a room temperature. A constant current is run through the coil. The heat dissipated by the coil increases the temperature of the environment. A set of 5 Z2-T-1M thermocouples (LABFACILITY, Bognor Regis, UK) is used to record the temperature at different locations around the coil. After approximately $30 \mathrm{sec}-$ onds, the coil reaches thermal equilibrium. We observe that for the thermocouple located the closest to the coil, the maximum temperature difference at that equilibrium is $15{ }^{\circ} \mathrm{C}$ for the current of $1.25 \mathrm{~A}$. The thermocouples located more distant from the coil recorded negligible temperature differences at all current values.

For the current of $0.75 \mathrm{~A}$ the coil temperature increases by $7{ }^{\circ} \mathrm{C}$. This value indicates that significant heating does take place. However, if the same increase was experienced in in vivo conditions, it has been shown to cause negligible tissue damage [200]. This allows us to speculate that, if operated at $0.75 \mathrm{~A}$, the prototype of tentacle catheter can be considered suitable for grasping tasks within human body. However, the heat transfer conditions can vary dramatically depending on the environment, in which the catheter is operated. Therefore, a more detailed safety study, involving heating of real human tissue kept at $37{ }^{\circ} \mathrm{C}$ is necessary to draw direct conclusions. For now, such investigation lies beyond the scope of our work.

\subsubsection{Estimation of Maximum Grasping Force}

Manipulation tasks require the tentacle catheter to apply force to the target object. The inherent compliance of the catheter imposes limits on the magnitude of that force. In our grasping strategy (Figure 5.3), we proposed using an electromagnetic coil to increase the maximum force, which can be applied to the object without deforming the catheter and breaking the contact. In this section we characterise the maximum applicable force, Furthermore, we investigate its relationship with the attraction force $\left(\mathbf{F}_{a}\right)$ between the tip magnet and the electromagnetic coil using FE simulation.

The force characterization experiment for the tentacle catheter is presented in Figure 5.6. The catheter is placed inside the workspace of BigMag - an array of mobile electromagnets [179]. Magnetic field generated by BigMag is used to wrap the catheter around a target object connected to K3D40 force sensor (Meßsysteme, Hennigsdorf, Germany). Once this task is finished, we activate the coil to form a loop around the target object. Subsequently, the catheter is retracted using an automated inserter. This results in a force being applied to the target object. This force is measured by the force sensor. Once the force exceeds a certain value, it breaks the connection between the tip magnet and the coil, releasing the target object. We determine this maximum applicable force by inspecting 

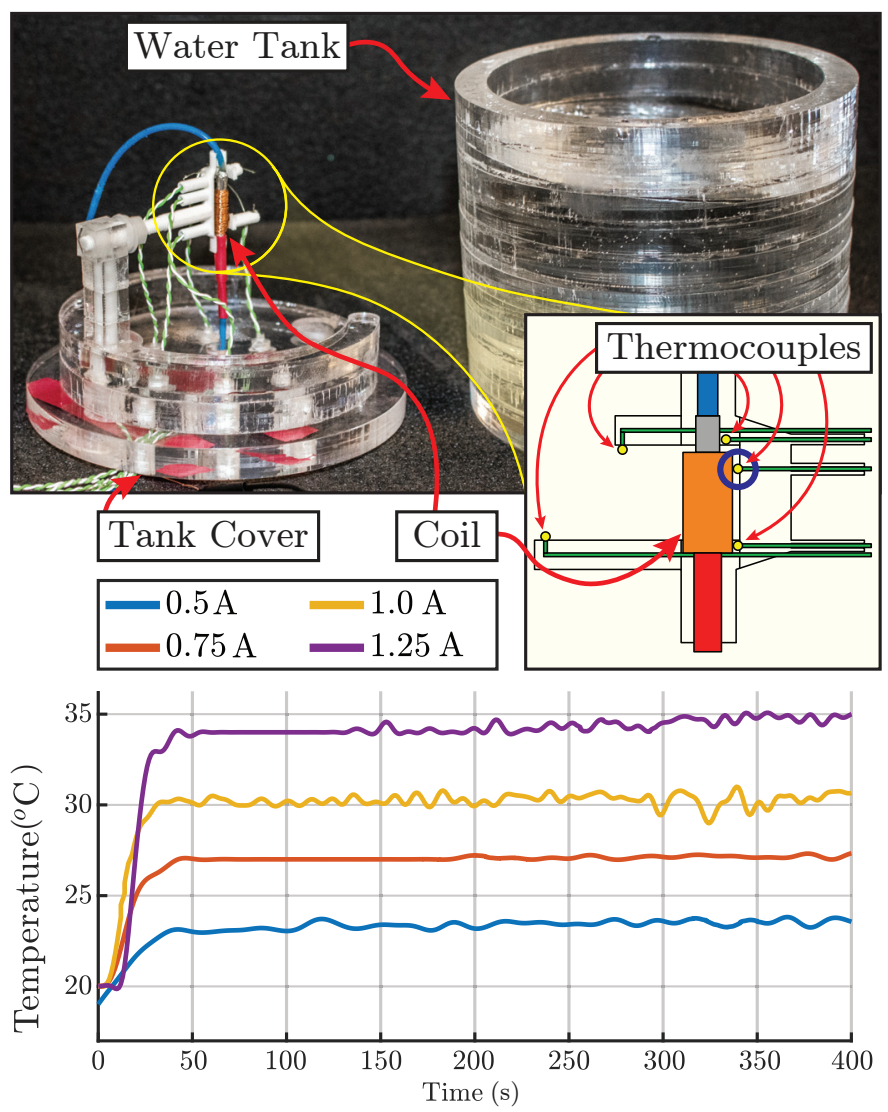

Figure 5.5: The thermal study performed to assess the safety of tentacle catheter. The prototype of the tentacle catheter shown in Figure 5.4 is held in a tank of water, which is initially at room temperature. 5 Z2-T-1M thermocouples (LABFACILITY, Bognor Regis, The UK) are used to record the temperature at different points around the coil of the catheter. Constant current of fixed magnitudes is run through the coil, which causes the coil temperature increase due to resistive heating. The temperature increase at four different values of current is recorded. The graph presents the readings from one thermocouple closest to the body of the coil (marked with purple circle in the figure). The temperature increase recorded further away from the coil was negligible in all cases.

the measurements from the force sensor. The experiment is repeated for four values of coil current. All the results are comparable, and lie within the range of 0.08-0.10 N. This force allows for manipulation against gravity of objects several orders of magnitudes heavier than the standard tissue samples extracted for during biopsies [207]. Therefore, we consider tentacle catheter to be clinically-relevant.

The dependence of the maximum applicable manipulation force on the attraction force $\left(\mathbf{F}_{a} \in \mathbb{R}^{3}\right)$ between the tip magnet and the electromagnetic coil is verified using finite element simulations (Figure 5.7). The model used for the simulation involves a coil and a permanent magnet kept at a distance $\left(d=0.15 \mathrm{~mm} \in \mathbb{R}^{+}\right)$ from one another. The magnet is kept at an angle $(\theta \in \mathbb{S})$ from the long axis of the coil. COMSOL 5.2 (Comsol Inc., Burlington, USA) is used to compute the value of $\left(\mathbf{F}_{a}\right)$ at a range of coil currents and angles $(\theta)$. 

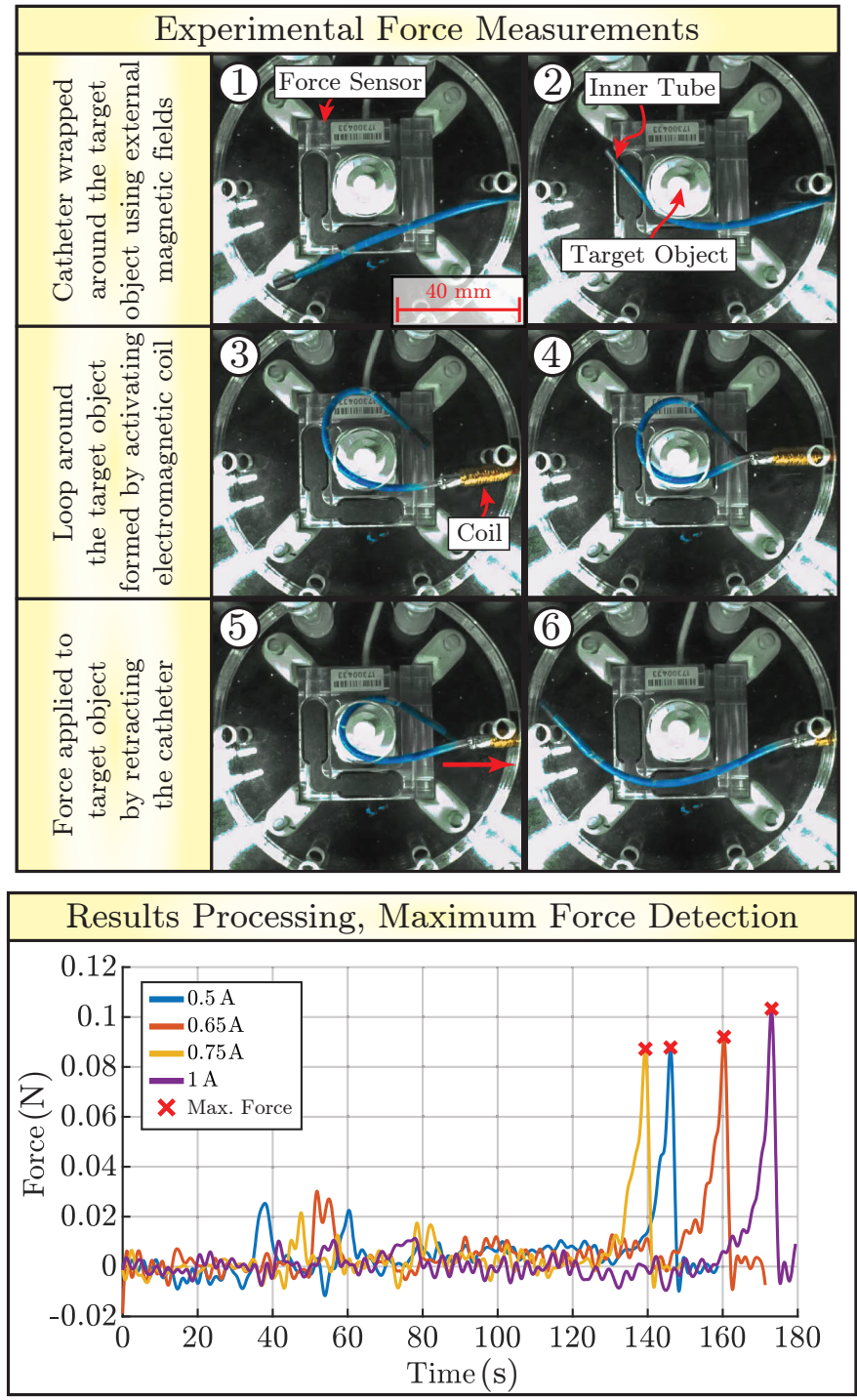

Figure 5.6: Experiment used to determine the maximum force applicable with tentacle catheter. The catheter is inserted into the workspace of BigMag array using an automated inserter (1). External magnetic field is used to wrap the catheter around a circular target object attached to K3D40 force sensor (Meßsysteme, Hennigsdorf, Germany) (2) - (3). After the activation of the coil, the catheter forms a stable loop around the object (4). Following this, the catheter is slowly retracted, applying force to the target object (5). If the force applied to the object is too large, the loop breaks and the catheter detaches from the object (6). The experiment is repeated for 4 values of coil currents. The maximum forces applicable with the tentacle catheter are within the range of 0.08-0.1 N.

We assume that only the component of $\mathbf{F}_{a}$ along the axis of magnet dipole moment $(\boldsymbol{\mu})$ plays role in stabilising the loop. The value of that component is compared against the experimental results. We observe close correspondence of 

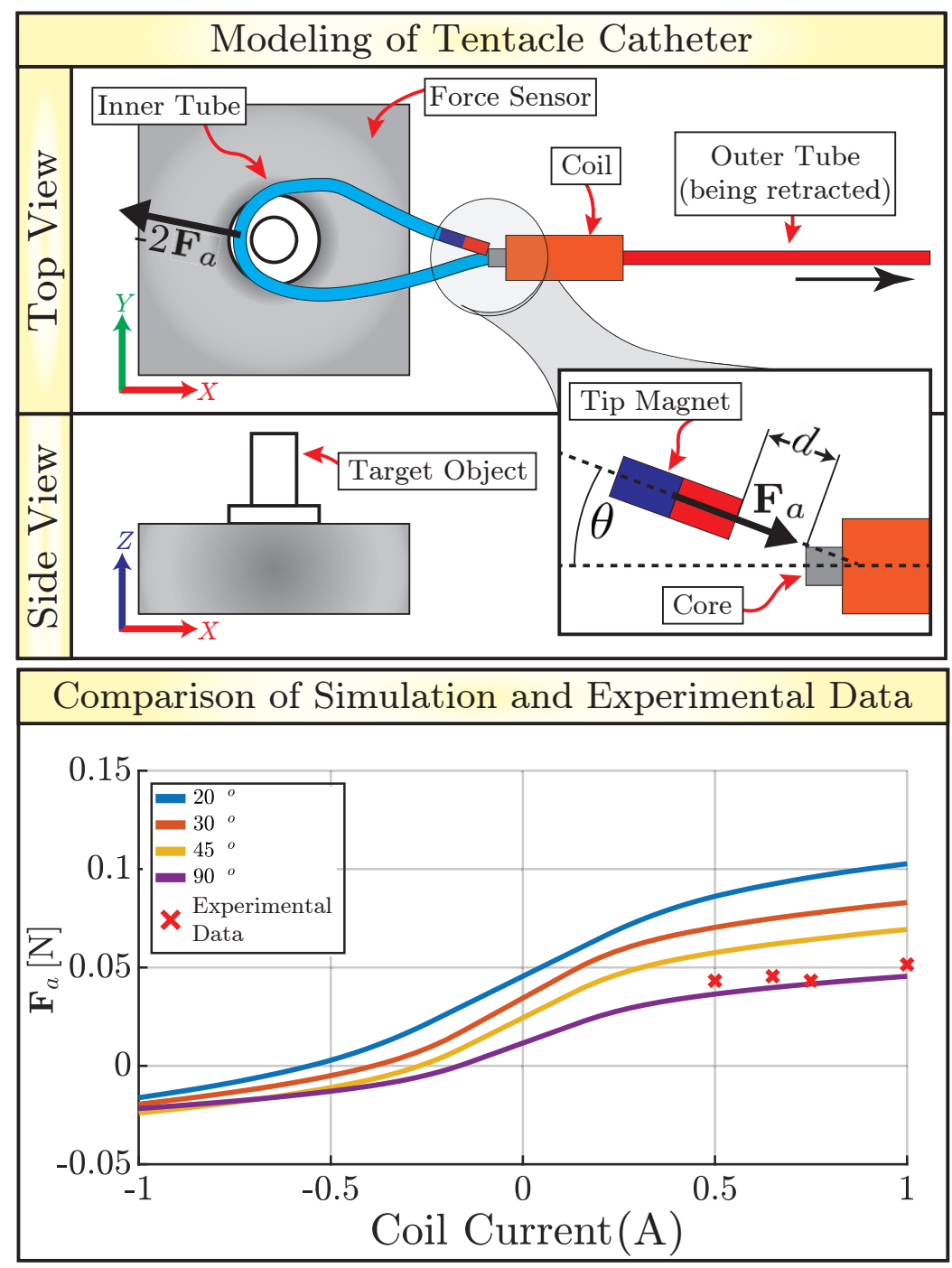

Figure 5.7: The relation between the attraction force $\left(\mathbf{F}_{a}\right)$ and the maximum force applicable by the tentacle catheter is investigated using finite element simulation. COMSOL 5.2 (Comsol Inc., Burlington, USA) is used to model the attraction between a permanent magnet located at the tip of the catheter and the electromagnetic coil. The assumed distance $\left(d \in \mathbb{R}^{+}\right)$between the magnet and the coil is $0.15 \mathrm{~mm}$, which accounts for thickness of non-magnetic coating. The attraction force $\left(\mathbf{F}_{a}\right)$ is computed for 4 different values of angle $(\theta \in \mathbb{S})$ between the magnet and the coil. The values of the component of $\mathbf{F}_{a}$ oriented towards the coil are compared against the maximum applicable forces (red crosses) determined experimentally (Figure 5.6). The results show that the maximum force which can be applied using the tentacle catheter is directly related to the magnitude of the attraction force $\left(\mathbf{F}_{a}\right)$ between the tip magnet and the electromagnetic coil. Furthermore, the predicted force value from the proposed attraction model closely matches experimental observations.

the forces measured in Figure 5.6 and the computed magnitudes of attraction force. Nevertheless, as the exact values of simulated force depend on the angle 
between the permanent magnet and the coil, which cannot be established without a tracking system, we are unable to quantify the exact discrepancy. Furthermore, the simulation predicts that approximately $0.5 \mathrm{~A}$ of current in the reverse direction is needed to break the connection between the tip magnet and the coil. This value matches with experimental observations.

\subsubsection{Pick-and-Place Task}

The final proof-of-concept experiment employs the tentacle catheter for manipulation of porcine tissue (Figure 5.8). It serves to demonstrate that the grasping strategy presented in Figure 5.3 is applicable to pick-and-place tasks involving biological material. The tentacle catheter is operated at $0.75 \mathrm{~A}$, as this current has been established safe by thermal study. The $20 \times 20 \times 20 \mathrm{~mm}$ sample of pork tissue is placed inside the workspace of BigMag. The mass of the sample is $9.5 \mathrm{~g}$. The tentacle catheter is wrapped around the tissue, the coil is activated to form a stable loop around it. Subsequently, the inserter of BigMag is used to retract the catheter, displacing the tissue. During the motion, reliable grasp has been observed. After the motion is finished, the current in the coil is reversed to release the catheter, which is later fully retracted from the workspace.

\subsection{Conclusions and Future Work}

In this chapter we present a proof-of-concept study of a magnetically-actuated tentacle catheter for grasping tasks within human body. The tentacle catheter is selected out of three concepts, based on its adherence to a set of design requirements. The prototype of the catheter is tested within the BigMag coil array. Since the heat dissipated by the catheter could potentially damage biological tissue, we test the safety of the catheter in a thermal study. Moreover, we show that using our proposed grasping technique, forces of up to $0.1 \mathrm{~N}$ can be applied for object manipulation. This force is in agreement with theoretical predictions. This force allows for manipulation of clinially-relevant samples of tissues. Finally, we test the catheter by successfully accomplishing a pick-and-place task using porcine tissue. 


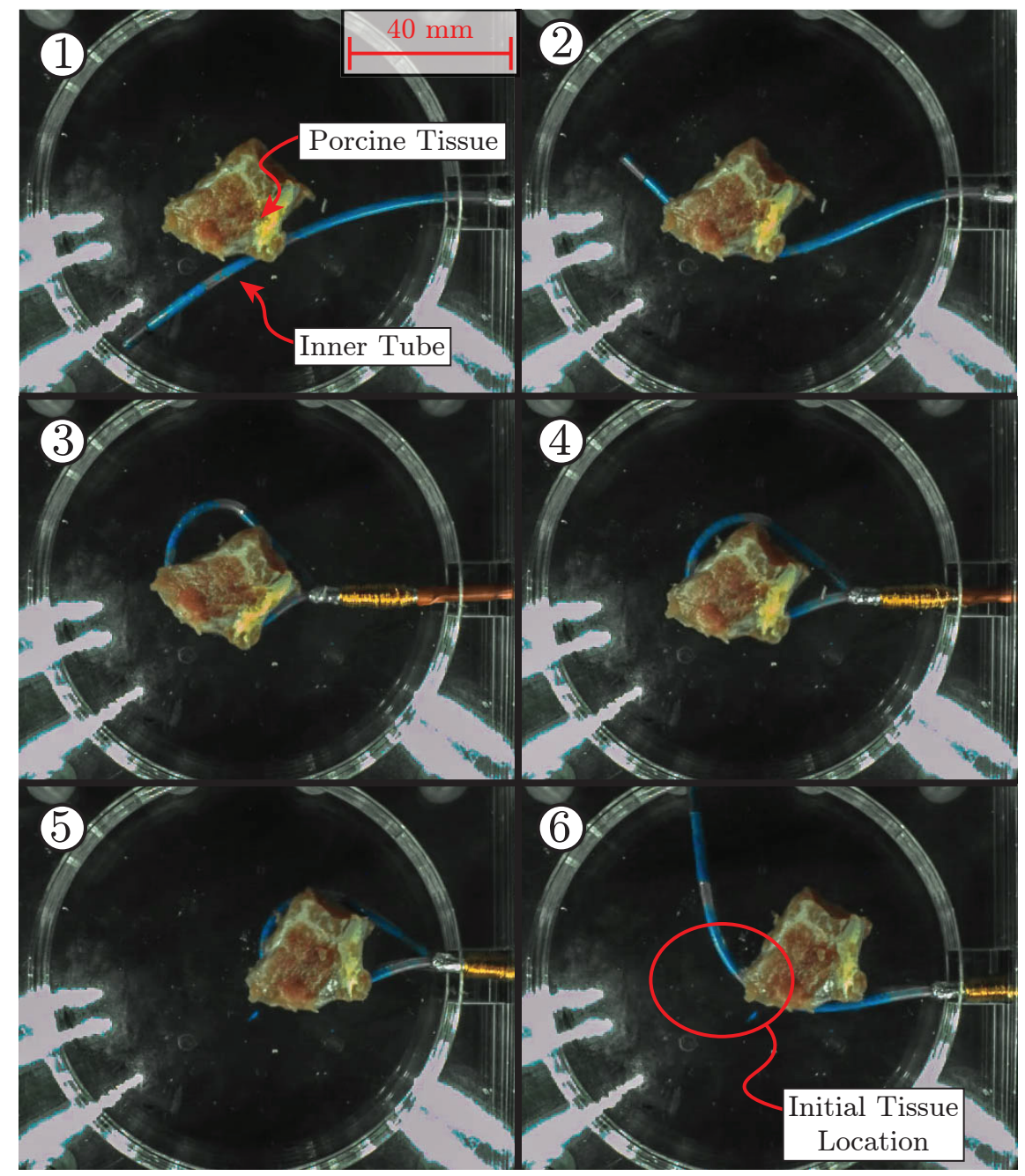

Figure 5.8: The final experiment involving execution of a pick-and-place task using tentacle catheter. The catheter is used to grasp a $20 \times 20 \times 20 \mathrm{~mm}$ piece of porcine tissue located inside the workspace of BigMag array. Subsequently, the BigMag inserter has been used to move the object by retracting the catheter. After reaching the desired location, the current in the coil of tentacle catheter has been reversed to release the grasp. 


\title{
Chapter 6
}

\section{Flexible Catheter for Ultrasound-Guided Delivery of Magnetic Projectiles}

\begin{abstract}
Magnetic actuation is a versatile technology, widely applied in medical robotics for non-contact steering of flexible instruments and untethered agents. In this chapter, we exploit the benefits of this technology by developing a magneticallyactuated flexible catheter capable of the controlled release and retrieval of untethered magnetic capsules. The catheter is actuated by the Advanced Robotics for Magnetic Manipulation (ARMM) system. The scanning ultrasound is used for $3 \mathrm{D}$ shape reconstruction. Using a position controller, we navigate the catheter to a final position, following which the capsule is ejected and moves as a projectile to the target. The trajectory of the projectile is defined before launch, using a targeting algorithm, which reconfigures the ARMM electromagnet, while keeping the catheter tip steady. The accuracy of our ultrasound shape reconstruction is measured, with the lowest error of $0.37 \mathrm{~mm}$. The closed-loop position controller is used to track a series of trajectories with the lowest mean error of $0.82 \mathrm{~mm}$. The targeting algorithm allows us to successfully launch the projectile to a designated target while limiting the residual tip displacement to $0.88 \mathrm{~mm}$.
\end{abstract}

Adapted from:

J. Sikorski, C.M. Heunis, R. Obeid, V. Kalpathy Venkiteswaran, S. Misra. Magnetic Catheter for Projectile-Based Ultrasound-Guided Targeted Drug Delivery, IEEE Transactions on Robotics [Submitted] 


\subsection{Introduction}

In the last decade, magnetic actuation has risen up as one of the most promising technologies for non-contact steering of robotic medical devices. The capability of remotely exerting a magnetic wrench at any point within a given workspace has a potential of shifting the design paradigms for steerable medical instruments. By employing magnetic actuation, instruments for minimally-invasive surgery (MIS) can be made safer, smaller, and more dexterous [134, 208].

The advantages of magnetic actuation are particularly prominent in the field of flexible surgical instruments, where it has been employed to create steerable catheters which are not burdened with the problems of friction, hysteresis, and backlash inherent to classical tendon-driven actuation [65]. Several studies have demonstrated controlled motion of simple magnetic catheters designed for procedures within the cardiovascular system [116,136,141,194]. Furthermore, the recent surge of research in this field produced means through which magnetic catheters can enable a whole new group of MIS procedures. Examples include catheters with controllable mechanical properties or functionalised elements, allowing tasks such as grasping, cutting or tissue coagulation [136, 145, 209,210].

Concurrently with the research on flexible catheters, the use of small-scale untethered agents as novel surgical tools has also attracted abundant scientific attention, due to the ability of these agents to interact with the human body at submillimeter scale [105]. Magnetic actuation is one of the key technologies enabling non-contact controlled movement of these agents [211]. Specifically, magnetic untethered milli-/microrobotic agents have been demonstrated to perform various clinically-relevant tasks such as targeted drug delivery, grasping or tissue penetration [212-214]. A general, extensive review of current advances in the field of medical milli-/microrobotics is presented in [104].

Despite the clinical potential demonstrated by magnetic catheters and untethered milli-/microrobotic agents in numerous studies, the practical applications of these devices are limited by a set of challenges. Magnetic catheters can be regarded as continuum robots - burdened with complex, high-bandwidth dynamical behaviour due to their continuously bending structure [41]. Control of this behaviour is challenging, yet crucial in assuring adequate reach, precision and stability of magnetic catheters, particularly at large deflections and higher velocities $[116,194]$. However, once the control of the trajectory of the distal end (tip) of a catheter is achieved, the rest of its body tends to follow that trajectory closely [215]. This allows the catheter to traverse large distances inside the cavities of human body, relying on compliance matching for safety [123]. Conversely, motion control over large distance is difficult for untethered milli-/microrobotic agents. In turn their positioning inside the final workspace can be achieved relatively precisely once they are successfully delivered there [216].

This complementary characteristics of catheters and milli-/microrobotic agents offers possibilities for development of a novel class of magnetic surgical instruments, which combine the elements of both. These surgical instruments can be actuated affectively due to the versatility and the universality of magnetic actuation. A recent study, presenting a mobile, yet tethered magnet as a tool for force control could be considered an early example of this approach [144]. Nevertheless, 
to the authors' best knowledge, the area remains unexplored in terms of advanced catheter designs capable of autonomous operations in clinically relevant tasks.

In this chapter we fill that gap by presenting a design of a catheter with a magnetic tip capable of controlled release and retrieval of millimeter-scale capsules (Figure 6.1). We use our catheter to realise a proof-of-concept multistage task, whereby a capsule is delivered into the workspace, and released as a projectile towards a target. We discuss, how this task can be exploited for targeted drug delivery.

We test our catheter in a clinically-relevant framework for catheter steering, which employs the mobile electromagnetic coil of the Advanced Robotics for Magnetic Manipulation (ARMM) system [217]. However, the clinical relevance of the mobile electromagnet approach comes at the price of increased complexity of the resulting system in comparison to classical, static coil arrays [99,110]. Despite the successful use of the ARMM system in ultrasound-guided 2D positioning tasks of catheter tip, full three-dimensional position control of a magnetic catheter using a mobile electromagnet has not yet been demonstrated [115].

The main contributions of this chapter are summarized below:

1. Design and prototyping of an advanced magnetic catheter capable of controlled release and retrieval of an untethered millirobotic capsule with biodegradable outer shell.

2. Significant redevelopment of a closed-loop controller for $3 \mathrm{D}$ steering of catheter tip proposed in Chapter 4. The controller presented in this chapter employs a novel method, whereby the mobile coil of the ARMM system is used for magnetic actuation.

3. Creation of a targeting algorithm for the guidance of an untethered agent released from a magnetic catheter as a projectile. The algorithm uses the nullspace of the primary control system to reconfigure the pose of the ARMM coil, which determines the trajectory of a projectile.

4. Development and validation of a scanning ultrasound technique to reconstruct the $3 \mathrm{D}$ shape of a magnetic catheter. The shape reconstruction is used both for the purpose of closed-loop control of the catheter, as well as for projectile targeting.

5. Experimental demonstration of our catheter under the guidance of scanning ultrasound. The demonstration involves two studies. The first study presents the position control of catheter tip across a set of trajectories. The second study involves the use of our targeting algorithm for successful delivery and retrieval of an untethered capsule to a predefined target.

The rest of this chapter is structured as follows. Section 6.2 introduces the design of our catheter for the delivery of untethered capsules. In Section 6.3 the ultrasound tracking and reconstruction techniques are described in more details. Section 6.4 contains the mathematical models used in our procedure and demonstrates how they can be used to derive a closed-loop control framework. Section 6.5 describes the test-bed used in the experimental studies executed to validate our 


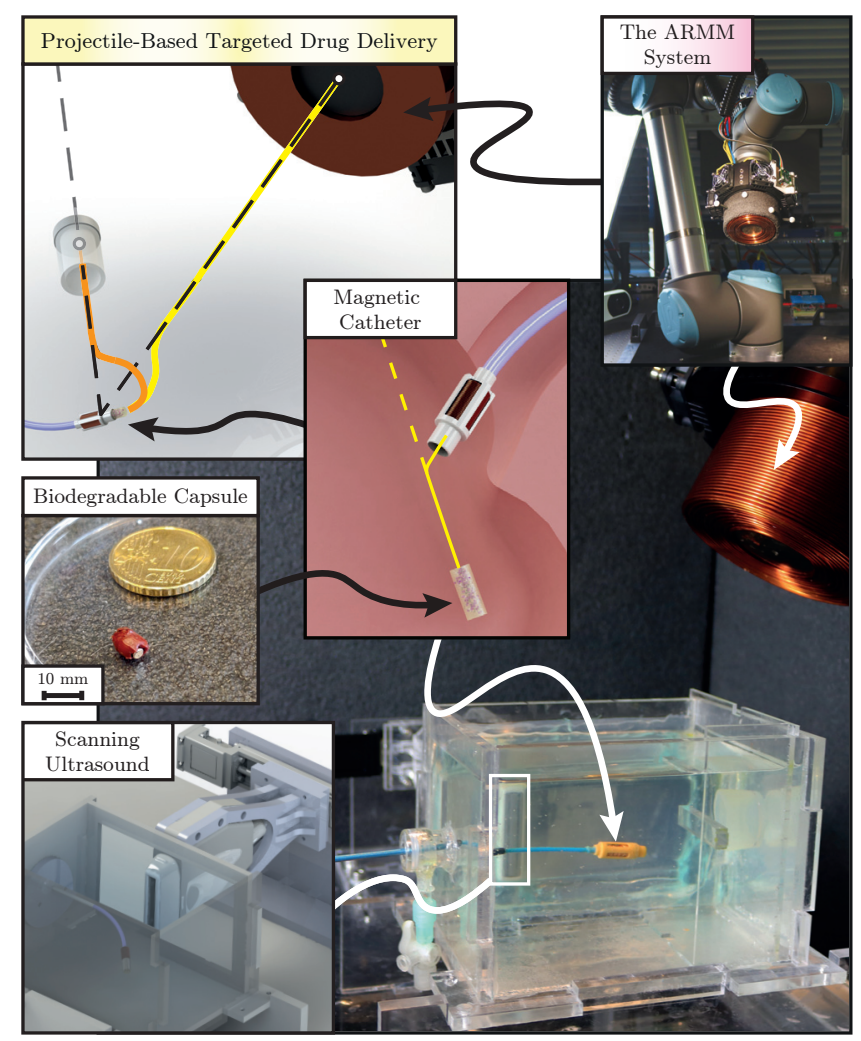

Figure 6.1: In this chapter we present a magnetic catheter for targeted drug delivery under the guidance of scanning ultrasound. The catheter employs a miniaturized magnetic coil in the proximal part of its tip and a dock for biodegradable magnetic capsules in a distal part. The position of the catheter tip is controlled using the external magnetic field provided by the ARMM (Advanced Robotics for Magnetic Manipulation) system. At the beginning of the procedure, the dock on the tip is loaded with a capsule. Active miniaturized coil keeps the capsule in position The catheter is steered to the target site using scanning ultrasound for shape reconstruction and the ARMM system for magnetic actuation. Upon reaching the target site, the capsule is ejected, by reversing the direction of the current and moves as a projectile towards the electromagnet of the ARMM system (yellow line). Since our system is overactuated, the trajectory of the projectile can be controlled to reach the target (orange line).

concept. The results of these studies are presented in Section 6.6 and discussed in Section 6.7. Finally, Section 6.8 concludes the chapter, providing directions for future research.

\subsection{Targeted Drug Delivery Using Magnetic Catheter}

The system presented in this chapter exploits the principles of magnetic actuation. This technique utilises external magnetic field $\left(\mathbf{B}(\mathbf{p}) \in \mathbb{R}^{3}\right)$ to generate magnetic wrenches $\left(\mathbf{w}_{\mu} \in \mathbb{R}^{6}\right)$ acting on a magnetic object located at a point $\left(\mathbf{p} \in \mathbb{R}^{3}\right)$. If the magnetic object is represented by its dipole moment $\left(\boldsymbol{\mu} \in \mathbb{R}^{3}\right)$, 
the resulting magnetic wrench, comprising of the magnetic torque $\left(\boldsymbol{\tau}_{\mu} \in \mathbb{R}^{3}\right)$ and force $\left(\mathbf{f}_{\mu} \in \mathbb{R}^{3}\right)$ is described by (1.1).

\subsubsection{System Design}

Remarkably, since magnetic actuation is universal, the relation (1.1) holds for a given object regardless of the source of the external field/gradient. This allows for magnetic elements to be used for different purposes within the same surgical system. The resulting instrument designs can express complex mechanical behaviour suitable for complex tasks, while retaining simple structure and small footprint facilitating minimally-invasive access [134]. We demonstrate the potential of such devices by creating a novel magnetic catheter, capable of controlled release and retrieval of untethered capsules. The catheter is intended to realise a task of targeted drug delivery (Figure 6.2, Top).

The magnetic capsule is located at the tip of the catheter in a rigid dock at its distal end. The capsule is made of a biodegradable matrix surrounding a permanent magnet with a dipole moment $\left(\boldsymbol{\mu}_{t} \in \mathbb{R}^{3}\right)$. Additionally, at its proximal end, the catheter tip contains an electromagnetic coil with a ferrous core. Current $\left(I_{c} \in \mathbb{R}\right)$ in this coil creates a dipole moment $\left(\boldsymbol{\mu}_{c} \in \mathbb{R}^{3}\right)$. We exploit the interaction between the dipoles of the coil and the capsule with one another and with the external magnetic field to realise a targeted drug delivery procedure comprising of two stages. (Figure 6.2, Bottom)

The magnetic attraction between the two dipoles is used to stabilise the position of the capsule throughout the first stage of our procedure, which involves navigation of the catheter to the target site. Since the coil and the capsule are effectively a part of the same rigid body, we consider their combined dipole moment $(\boldsymbol{\mu})$ for steering of catheter tip position. We use a source of external magnetic field to apply magnetic wrenches $(\mathbf{w})$ to this dipole moment. These wrenches are used to deflect the catheter, making its tip follow a prescribed trajectory. Given that sensing measures are in place for tip position feedback, the task of reaching the target site can be then realised by a closed-loop position controller.

Upon reaching the target area, the second stage of our procedure can take place. Since the catheter by itself has limited manoeuvrability, its effective reach is limited. We overcome this limitation by turning the capsule into an untethered agent. Once the tip is close to the target, we eject the capsule from the dock by reversing the current $\left(I_{c}\right)$, which creates a strong repulsive force. Upon release, the capsule becomes a projectile, which is guided towards the final location using an external magnetic field.

The strategy assumes that the active substance is released by biodegradation of the outer shell of the projectile. After the successful release of the substance, the remainders of the projectile are removed from the target site. For that purpose, we also use the miniaturized coil incorporated on the catheter tip. By reversing the direction of the external magnetic force, we guide the projectile into the vicinity of the catheter tip. The projectile attracts to the miniaturized electromagnet and the entire system can be retracted from the workspace. 


\subsubsection{Prototype Fabrication}

Our catheter is validated in a proof-of-concept study involving a functional prototype. In this work we focus on the robotic aspect of the procedure, involving the delivery and retrieval of the capsule. The controlled substance release associated with the biodegradation of the capsule remains outside the scope of this chapter and will become the subject of future studies.

The prototype (Figure 6.2) comprises of a magnetic tip mounted onto the flexible shaft extruded from Pebax 6333 thermoactive polymer (length $60 \mathrm{~mm}$, $\varnothing 2 \mathrm{~mm}$ ). The outer shell of the end-effector is fabricated from a photoactive resin using stereolitographic 3D printing (Prusa SL1, Prusa Research, Prague, Czech Republic). In the proximal compartment of the end effector, we locate a custommade copper-wire electromagnetic coil with stainless steel core (coil $\varnothing 6 \mathrm{~mm}$, core $\varnothing 3 \mathrm{~mm}$, wire $\varnothing 0.25 \mathrm{~mm}$, length $10 \mathrm{~mm}$, resistance $1.1 \Omega$ ) powered by insulated leads, which are fixed inside the flexible shaft. The coil can run currents of up to \pm 3 A, supplied by an iPOS4808 BX-CAT current controller (Technosoft Motion, Neuchatel, Switzerland).

The capsule in the proof-of-concept study comprises of a cylindrical permanent magnet (length $6 \mathrm{~mm} \varnothing 2 \mathrm{~mm}$, Webcraft GmbH, Gottmadingen, Germany) surrounded by a biodegradable, drug-releasing composite (Figure 6.3). We simulate the active substance (drug) with Sudan Orange dye (Boom B.V, Meppel, Netherlands) embedded in a carrier biodegradable gelatine matrix (Boom B.V., Meppel, Netherlands). The capsules are fabricated in a series of steps. First, a cuboidal Poly(Methyl Methacrylate) (PMMA) mould is fabricated using laser cutting technique. A stack of permanent magnets is fitted in the middle of the die. Gelatin, water and the Sudan Orange dye are mixed in proportion (12:100:1) and heated to $90{ }^{\circ} \mathrm{C}$. The mixture is poured into the mould and allowed to set for 12 hours. The cuboidal blocks are taken out of the mould and dried, which decreases the size of the biocomposite layer and improves its binding to the permanent magnets. Finally, dry blocks are divided into individual capsules, loaded into the dock of the catheter and used as projectiles in the delivery procedures.

\subsection{Catheter Tracking}

As our magnetic catheter is designed to operate within the confines of the human body, a clinically-relevant feedback modality must be available, providing information about the configuration and location of the catheter for the purpose of closed-loop control. For that purpose, we employ ultrasound. During the navigation and targeting stages of the delivery we acquire volume scans of the catheter within its environment using a mobile 2D ultrasound probe. Subsequently, the scans are processed to segment the silhouette of the catheter and retrieve its mechanical configuration.

To realise this method, we propose a a custom-build scanner system (Figure 6.4). The scanner comprises of a $14 \mathrm{MHz}$ L14-5/38 2D ultrasound transducer (Ultrasonix SonixTouchQ+, BK Medical, Peabody, MA, USA) on a motorized linear stage. By controlling the position of the DC motor, the transducer can be linearly displaced along the workspace. 


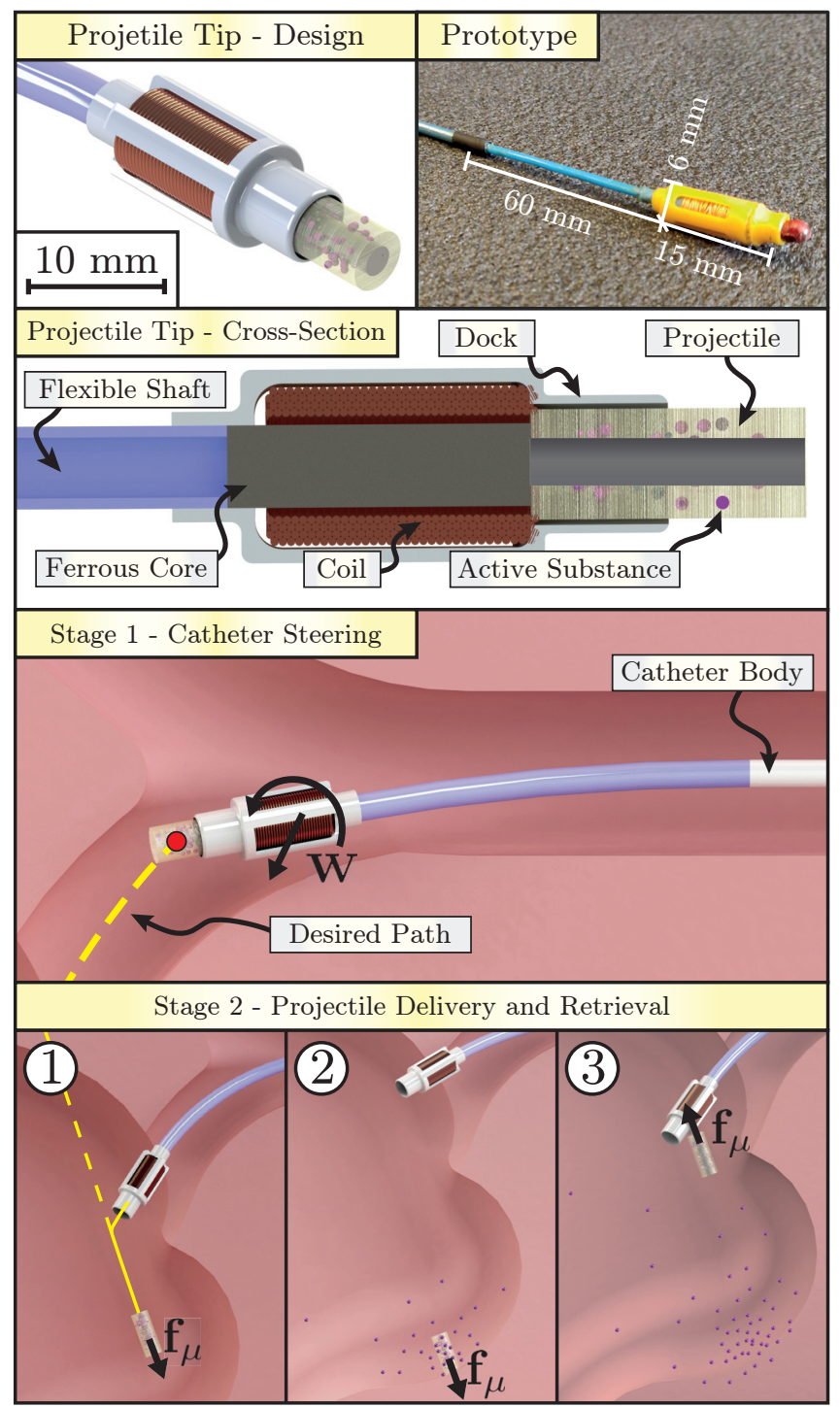

Figure 6.2: We propose to realise targeted drug delivery using a flexible catheter carrying a capsule with a centrally located permanent magnet (grey) and the active substance (purple) embedded in biodegradable matrix. The capsule is fixed in a dock within the end-effector of the catheter, which also contains a miniaturized electromagnetic coil. In the first stage of the procedure, the coil and the capsule are attracted to one another and can be considered a single magnetic element. This element is actuated using magnetic wrenches $\left(\mathbf{w} \in \mathbb{R}^{6}\right)$, which are used to guide the catheter to the target site. Once close to the target, the capsule is released by changing the direction of the current in the miniaturized coil. The ejected capsule is moves as a projectile under the influence of external magnetic force $\left(\mathbf{f}_{\mu} \in \mathbb{R}^{3}\right)$. (1) The direction of that force (yellow) determines the trajectory of the projectile. (2) Once at the target site, the projectile is held in place using magnetic force, while its biodegradable structure decomposes, releasing the active substance. (3) After the release, the permanent magnet is removed from the target site by attracting it back to the coil of the catheter by reversing the direction of $\mathbf{f}_{\mu}$. 


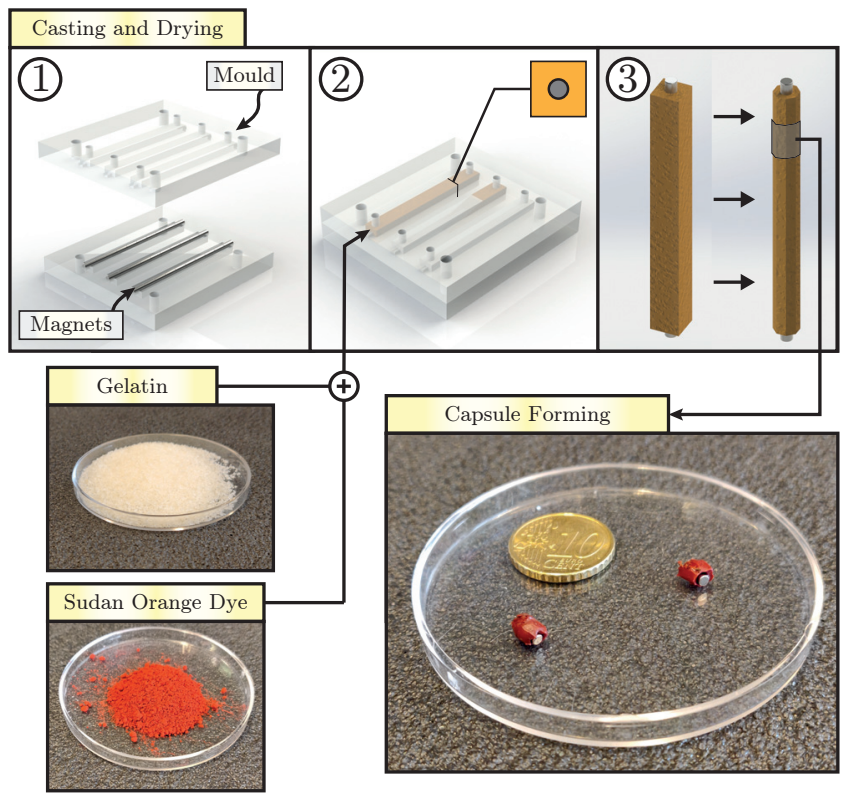

Figure 6.3: The capsules used as projectiles in the targeted drug delivery method proposed in this chapter comprise of permanent magnets (length $6 \mathrm{~mm} \varnothing 2 \mathrm{~mm}$, Webcraft GmbH, Gottmadingen, Germany) surrounded by biodegradable, drug-releasing composite. The composite employs gelatin (Boom B.V., Meppel, Netherlands) to suspend particles of drug-imitating Sudan Orange dye (Boom B.V., Meppel, Netherlands). The capsules are fabricated using laser-cut PMMA moulds. (1) Stacks of permanent magnets are fixed in the centre of the moulds. (2) Subsequently, the moulds are closed and filled with a mixture of water, gelatin and Sudan Orange dye (proportions 100:12:1). The casts are allowed to set. After that they are extracted and dried, which improves the binding between the magnet and the biocomposite and decreases the total volume of the cast. Finally, the cast is divided into individual capsules, each containing a single permanent magnet.

During the catheter navigation, the scanner sweeps the transducer periodically between two position limits, producing two-dimensional B-mode images at $36 \mathrm{~Hz}$ (Figure 6.4). A stack of these images, along with their locations expressed in workspace frame of reference $\left(\left\{\mathcal{G}^{\prime}\right\}\right)$, is recorded for each sweep of the scanner. The silhouette of the catheter is located on each B-mode image using a template tracker described by Kaya et al. [218]. The pixels comprising the silhouette are segmented based on a constant threshold value. Pixels from all images are assembled into a point-cloud using the information about the position of each frame within the stack.

For PRB model reconstruction from the point-cloud we used an iterative algorithm, the details of which have been presented in Chapter 4. The algorithm provides a set of points lying approximately along the centreline of the catheter. A sixth-order polynomial is fitted to this set, which allows us to represent the shape of the catheter as a continuous function $\left(\mathbf{P}(s): \mathbf{R}^{+} \mapsto \mathbf{R}^{3}\right)$. This polynomial function is subsequently processed to retrieve PRB parameters $\left(\mathbf{q}_{c}\right)$ for the purpose of catheter steering. 


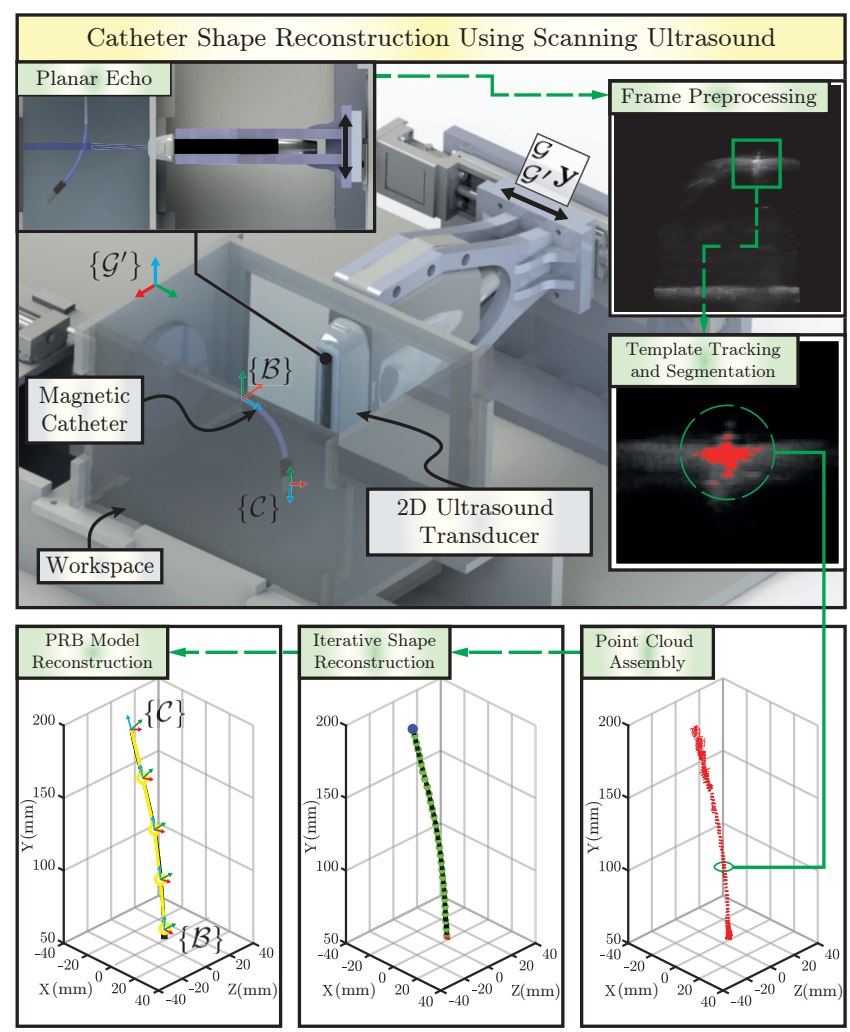

Figure 6.4: The pseudo-rigid-body (PRB) configuration of the catheter is reconstructed by using volumetric ultrasound scan technique. A $14 \mathrm{MHz}$ L14-5/38 2D ultrasound transducer of the Ultrasonix SonixTouchQ+ system (BK Medical, Peabody, MA, USA) is located in the workspace and registered with respect to its frame of reference $\left(\left\{\mathcal{G}^{\prime}\right\}\right)$. The transducer is periodically swept along $\left({ }_{\mathcal{G}^{\prime}}^{\mathcal{G}} \widehat{\mathbf{y}} \in \mathbb{R}^{3}\right)$. A stack of position-ordered two-dimensional ultrasound frames is collected during a single sweep. The silhouette of the catheter, spanning between the frames at its base $(\{\mathcal{B}\})$ and tip $(\{\mathcal{C}\})$ is extracted from the stack iteratively, by a template-based tracker similar to [218]. The pseudo-rigid-body model is then reconstructed from the resulting point-cloud by using the technique presented in Chapter 4

\subsection{Magnetic Catheter Steering Using the ARMM System}

The steering of the catheter presented in the previous sections requires a source of external magnetic field $(\mathbf{B}(\mathbf{p}))$. The classical magnetic actuation systems, based on the arrays of small-scale coils, usually have much higher bandwidth than the catheter. This enables their successful operation with relatively simple modelling and little concern about configuration-specific constraints of magnetic field generation [99].

Nevertheless, the clinical applicability of such systems is limited due to poor scalability resulting in small effective workspaces. Thus, for the purpose of this study, we integrate our catheter within the ARMM system (see Figure 6.5). This system uses a mobile coil fixed on a UR10 robotic arm (Universal Robots, Odense, 
Denmark), being capable of generating $\mathbf{B}(\mathbf{p})$ in workspaces spanning the entire human body. A detailed description of the system and the rationale behind its design are presented in Chapter 3 .

The use of mobile coils solves the problems of classical static coil arrays, albeit at an expense of increased mathematical complexity of the underlying field generation technique. In this section we propose a method for using the ARMM system for robotic steering of our magnetic catheter. First, we employ a reduced-order pseudo-rigid-body (PRB) models to derive the closed-loop control scheme, which enables autonomous steering of our catheter. Subsequently, we present a method of targeting the projectile capsule towards the target during the delivery phase. For that purpose, we reconfigure the ARMM system using its null-space, such that the projectile follows a trajectory that allows it to reach the target.

\subsubsection{Stage 1: Catheter Navigation}

During the first stage of the drug delivery procedure, the catheter is navigated using the ARMM system to a target site. We model the procedure, considering four principal coordinate reference frames. The frames are shown in Figure 6.5. The global reference frame $(\{\mathcal{G}\})$ is located at the base of the robotic arm. The generalised coordinates of the UR10 joints $\left(\mathbf{q}_{m} \in \mathbb{S}^{6}\right)$ define the pose of the end-effector frame $(\{\mathcal{T}\})$ located on the distal face of the electromagnet. The mathematical description of the catheter uses two frames: $\{\mathcal{B}\}$ at its base and $\{\mathcal{C}\}$ at its tip.

The linear transformation between any two frames $(\{\mathcal{J}\})$ and $(\{\mathcal{K}\})$ is represented by homogeneous transformation matrices $\left({ }_{\mathcal{K}}^{\mathcal{H}} \mathbf{H} \in \mathrm{SE}(3)\right)$. We define them as follows:

$$
{ }_{\mathcal{K}}^{\mathcal{J}} \mathbf{H}=\left[\begin{array}{cc}
\mathcal{J} \mathbf{R} & \mathcal{J}_{\mathcal{K}} \mathbf{p} \\
\mathbf{0}_{1 \times 3} & 1
\end{array}\right]
$$

where

$$
\begin{aligned}
& { }_{\mathcal{K}}^{\mathcal{R}}=\left[\begin{array}{lll}
\mathcal{J} \widehat{\mathcal{X}} & \mathcal{J} \widehat{\mathcal{Y}} & \mathcal{J}_{\mathcal{K}} \widehat{\mathbf{z}}
\end{array}\right] \in \operatorname{SO}(3), \\
& { }_{\mathcal{K}}^{\mathcal{J}}=\left[\begin{array}{lll}
\mathcal{J} & \mathcal{J}_{\mathcal{K}} y & \mathcal{J}_{\mathcal{K}} z
\end{array}\right]^{T} \in \mathbb{R}^{3}
\end{aligned}
$$

The matrices associated with the end-effector frame $(\{\mathcal{T}\})$ are calculated from instantaneous generalised coordinates $\left(\mathbf{q}_{m}\right)$ of the ARMM system [219]. The pose of the catheter is retrieved using the scanning ultrasound technique.

In our steering strategy the forward motion of the catheter is provided by an automated inserter, capable of applying arbitrary displacements $(\lambda \in \mathbb{R})$ along the neutral axis of the catheter $\left(\hat{\boldsymbol{\lambda}} \in \mathbb{R}^{3}\right)$. The deflection of the flexible body of the catheter away from that axis is achieved by using the magnetic field $\left(\mathbf{B}\left({ }_{\mathcal{C}}^{\mathcal{G}} \mathbf{p}\right)\right)$ and its gradient $(\nabla \mathbf{B})$ generated by the ARMM system to apply wrenches $\left(\mathbf{w}_{\mu}\right)$ acting at the tip dipole $(\boldsymbol{\mu})$ located at ${ }_{\mathcal{C}}^{\mathcal{G}}$ p. At any instance of time, the field/gradient depends on the configuration of the electromagnetic coil $\left({ }_{\mathcal{T}}^{\mathcal{G}} \mathbf{H}\right)$ and the current $(I \in \mathbb{R})$ running through it.

We model the catheter deflection due to $\mathbf{w}_{\mu}$ using a quasi-static PRB model presented in details in Chapter 4 . The continuously bending body of the catheter 


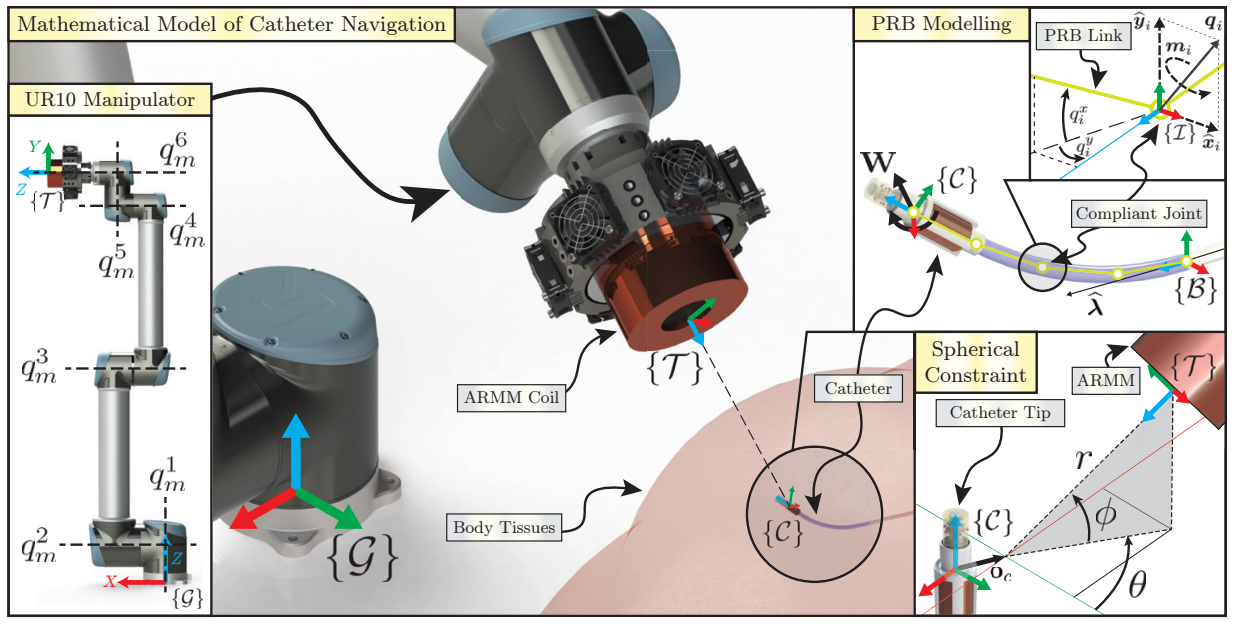

Figure 6.5: We demonstrate autonomous operation of our magnetic catheter using the ARMM system as the actuation framework. This system employs a 6DoF UR10 robotic arm (Universal Robots, Odense, Denmark) to position an electromagnetic coil within a large workspace. Magnetic field generated by the ARMM coil interacts with the dipole at the tip of the catheter, generating wrenches $\left(\mathbf{w} \in \mathbb{R}^{6}\right)$. In order to describe the effect of $\mathbf{w}$ on the shape of the catheter, we use the pseudo-rigid-body (PRB) model, expressed in catheter base frame $(\{\mathcal{B}\})$. This model represents the continuously bending catheter by a series of rigid links connected by compliant joints. Each joint $(i)$ is parametrised by two angles variables $\left(q_{i}^{x}, q_{i}^{y} \in \mathbb{S}\right)$, based on the internal bending moment $\left(\mathbf{m}_{i} \in \mathbb{R}^{3}\right)$ at the joint due to external load, including $\mathbf{w}$. The total deflection of all PRB joints determines the pose of the tip frame $(\{\mathcal{C}\})$. The actuation model is simplified by imposing a spherical constraint. The UR10 joint variables $\left(\mathbf{q}_{m} \in \mathbb{S}^{6}\right)$ are controlled in such a way, that the $Z$ axis of the ARMM tool frame $(\{\mathcal{T}\})$ always points towards catheter tip $(\{\mathcal{C}\})$, and the offset $\left(\mathbf{o}_{c} \in \mathbb{R}^{3}\right)$ is minimized. The resulting position of the ARMM coil can be parametrised in spherical coordinates $\left(\mathbf{s}=\left[\begin{array}{lll}r & \theta & \phi\end{array}\right] \in \mathbb{R}^{+} \times \mathbb{S}^{2}\right)$. By finding the relation between $\mathbf{s}$ and $\mathbf{w}$ the catheter can be remotely navigated in global reference frame $(\{\mathcal{G}\})$ using inverse model control, given appropriate feedback modality is present.

is divided into discrete, pseudo-rigid links, while its compliance is represented by torsional elements within 2DoF joints connecting the links. By assuming very slow motion of the catheter, we can neglect its transient dynamics [116]. Thus, we model its behaviour by a static equilibrium equation expressed in frame $\{\mathcal{C}\}$ as follows:

$$
\mathbf{J}_{c}^{T} \mathbf{w}=\mathbf{C} \mathbf{q}_{c}
$$

where $\mathbf{J}_{c} \in \mathbb{R}^{6 \times 6}$ is the body catheter Jacobian derived as in (4.11) from pseudorigid joint angles $\left(\mathbf{q}_{c} \in \mathbb{R}^{6}\right)$. The positive-definite matrix $\left(\mathbf{C} \in \mathbb{R}^{6 \times 6}\right)$ is used to model the compliance of the PRB joints. We define the effective wrench $\left(\mathbf{w} \in \mathbb{R}^{6}\right)$ as follows:

$$
\mathbf{w}=\mathbf{w}_{\mu}+\mathbf{w}_{g}
$$

to include the effect of both the magnetic wrench $\left(\mathbf{w}_{\mu}\right)$ and the gravity $\left(\mathbf{w}_{g} \in \mathbb{R}^{6}\right)$ due to the weight of the catheter tip.

We aim to use the PRB model (6.4) in a resolved-rate inverse model controller, establishing the relation between the ARMM control variables and the catheter 
tip velocity $\left({ }_{\mathcal{C}}^{\mathcal{G}} \dot{\mathbf{p}}\right)$. We differentiate $(6.4)$ to reach the following:

$$
\mathbf{J}_{c}^{T} \dot{\mathbf{w}}+\dot{\mathbf{J}}_{c}^{T} \mathbf{w}_{\mu}+\dot{\mathbf{J}}_{c}^{T} \mathbf{w}_{g}=\mathbf{C} \dot{\mathbf{q}}_{c} .
$$

The Jacobian derivative terms in (6.6) can be expressed linearly in $\dot{\mathbf{q}}$, such that

$$
\dot{\mathbf{J}}_{c}^{T} \mathbf{w}=\boldsymbol{\Gamma}\left(\mathbf{J}_{c}^{T}, \mathbf{w}\right) \dot{\mathbf{q}} .
$$

The transformation $\left(\boldsymbol{\Gamma}\left(\mathbf{J}_{c}^{T}, \mathbf{w}\right): \mathbb{R}^{6 \times 6} \times \mathbb{R}^{6} \mapsto \mathbb{R}^{6 \times 6}\right)$ is defined as follows [194]:

$$
\boldsymbol{\Gamma}\left(\mathbf{J}_{c}^{T}, \mathbf{w}\right)=\left[\begin{array}{c}
\frac{\partial J^{1,1}}{\partial \mathbf{q}_{c}} w_{1}+\cdots+\frac{\partial J^{6,1}}{\partial \mathbf{q}_{c}} w_{6} \\
\cdots \\
\frac{\partial J^{1,6}}{\partial \mathbf{q}_{c}} w_{1}+\cdots+\frac{\partial J^{6,6}}{\partial \mathbf{q}_{c}} w_{6}
\end{array}\right] .
$$

By combining (6.6) and (6.7), an analytic relation between the magnetic wrench rate $(\dot{\mathbf{w}})$ and catheter tip velocity becomes:

$$
\mathbf{J}_{a} \dot{\mathbf{w}}_{\mu}=\dot{\mathbf{p}}
$$

where

$$
\mathbf{J}_{a}={ }_{\mathcal{C}}^{\mathcal{G}} \mathbf{R}^{T} \mathbf{J}_{c, l}\left(\mathbf{C}-\boldsymbol{\Gamma}\left(\mathbf{J}_{c}, \mathbf{w}_{\mu}\right)-\boldsymbol{\Gamma}\left(\mathbf{J}_{c}, \mathbf{w}_{g}\right)\right)^{-1} \mathbf{J}_{c}^{T}
$$

and $\mathbf{J}_{c, l}$ is the linear component of the Jacobian matrix $\left(\mathbf{J}_{c}\right)$

Having independent control over the elements of wrench $\left(\mathbf{w}_{\mu}\right)$ would allow us for direct application of (6.9) in an inverse model controller similar to our previous approach [194]. Nevertheless, the ARMM system relies on controlled motion of the coil to generate the desired $\mathbf{B}(\mathbf{p})$, which prevents instantaneous, arbitrary variation of $\mathbf{w}_{\mu}$. Therefore, the behaviour of the ARMM system has to be explicitly taken into account, while designing the controller for our catheter. We divide the controller into two cascades, making the inner loop responsible for low-level motion of the UR10 described by a set of parameters. The outer loop will control these parameters to steer the tip position $\left({ }_{\mathcal{C}}^{\mathcal{G}} \mathbf{p}\right)$ along a trajectory. The entire control strategy is summarised in Figure 6.6.

At any given configuration, the effect of UR10 joint angles $\left(\mathbf{q}_{m}\right)$ and the mobile current $(I)$ on the magnetic wrench $(\mathbf{w})$ can be described by a magnetic field model. In our motion strategy, we employ the model associated with the actuation along the symmetry axis of the coil $\left({ }_{\mathcal{T}}^{\mathcal{G}} \widehat{\mathbf{z}}\right)$. If radius $\left(r \in \mathbb{R}^{+}\right)$denotes the distance from the face of the coil the magnetic field and its gradient along that axis expressed in frame $(\{\mathcal{T}\})$ can be computed as follows:

$$
\begin{aligned}
\mathbf{B} & =I\left[\begin{array}{lll}
0 & 0 & 1
\end{array}\right]^{T} b(r, I) \\
\nabla \mathbf{B} & =\operatorname{diag}\left(\left[\begin{array}{lll}
-0.5 & -0.5 & 1
\end{array}\right]\right) I \frac{\partial b}{\partial r},
\end{aligned}
$$

where $\left(b(r, I): \mathbb{R}^{+} \times \mathbb{R}^{+} \mapsto \mathbb{R}\right)$ is a non-linear field model [217]. More details of that model can be found in the Appendix. 


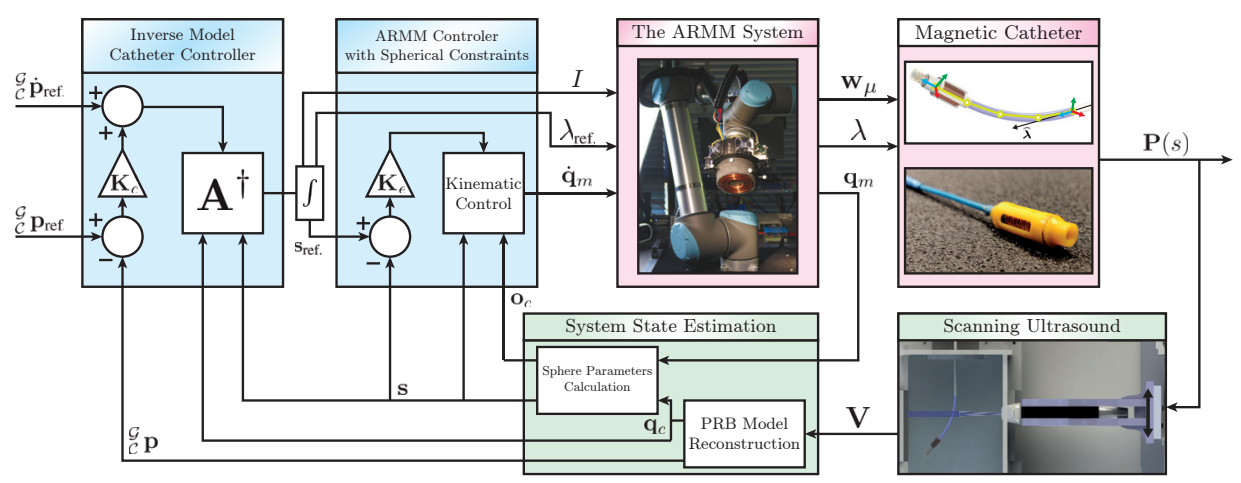

Figure 6.6: The control system (blue) proposed in this work is used to track the reference position of the magnetic catheter $\left({ }_{C}^{\mathcal{G}} \mathbf{p}_{\text {ref. }} \in \mathbb{R}^{3}\right)$. The catheter shape $(\mathbf{P}(s)$, defined as in Chapter 4$)$ is manipulated using the Advanced Robotics for Magnetic Manipulation (ARMM) system by exerting magnetic wrench $\left(\mathbf{w}_{\mu} \in \mathbb{R}^{6}\right)$ at the tip of the catheter and the displacement $(\lambda \in \mathbb{R})$ at its base. The behaviour of the ARMM system is driven by specifying the robot joint velocity $\left(\dot{\mathbf{q}}_{m} \in \mathbb{R}^{7}\right)$, reference inserter position $\left(\dot{\lambda}_{\text {ref. }} \in \mathbb{R}\right)$ and coil current $(I \in \mathbb{R})$. These values are computed in a two stage control approach. The inner controller enforces spherical constraint (Figure 6.5) with parameters $\left(\mathbf{s}_{\text {ref. }} \in \mathbb{R}^{+} \times \mathbb{S}^{2}\right)$. These parameters are calculated by the inverse model controller, which uses the linearised, reduced-order inverse model of the system $\left(\mathbf{A}^{\dagger} \in \mathbb{R}^{5 \times 3}\right)$ to minimize the error between ${ }_{\mathcal{C}}^{\mathcal{G}} \mathbf{p}_{\text {ref. }}$ and the actual position estimate $\left({ }_{\mathcal{C}}^{\mathcal{G}} \mathbf{p} \in \mathbb{R}^{3}\right)$. We provide the feedback to both control stages by tracking the shape of the catheter using linear scanning ultrasound technique. This technique generates a point cloud $(\mathbf{V})$, processed to retrieve the estimates of the pseudo-rigid-body configuration of the catheter $\left(\mathbf{q}_{c} \in \mathbb{R}^{8}\right)$. For the purpose of spherical constraint control, the actual sphere parameters $\left(\mathbf{s} \in \mathbb{R}^{+} \times \mathbb{S}^{2}\right)$ and the offset $\left(\mathbf{o}_{c} \in \mathbb{R}^{3}\right)$ between the centre of the sphere and the catheter tip are calculated using the information about ARMM and catheter configuration. The gain matrices $\left(\mathbf{K}_{c}, \mathbf{K}_{e} \in \mathbb{R}^{3 \times 3}\right)$ are used to tune the performance of both control stages.

For a dipole frame $(\{\mathcal{C}\})$ located on axis $\left(\mathcal{G}_{\mathcal{T}} \widehat{\mathbf{z}}\right)$, the position of the face of the coil can be parametrised by spherical coordinates

$$
\mathbf{s}=\left[\begin{array}{lll}
r & \theta & \phi
\end{array}\right] \in \mathbb{R}^{+} \times \mathbb{S}^{2},
$$

as shown in Figure 6.5. Subsequently, combining (1.1) and (6.11), the magnetic wrench $\left(\mathbf{w}_{\mu}\right)$ can be expressed in frame $(\{\mathcal{C}\})$ as a function of control parameters $(\mathbf{s}, I)$ as follows:

$$
\mathbf{w}_{\mu}(\mathbf{s}, I)=I \mu\left[\begin{array}{cc}
-\frac{3}{2} \sin \theta \sin \phi \cos \phi & 0 \\
\frac{3}{2} \cos \theta \sin \phi \cos \phi & 0 \\
\sin ^{2} \phi-\frac{1}{2} \cos ^{2} \phi & 0 \\
0 & -\cos \theta \cos \phi \\
0 & -\sin \theta \cos \phi \\
0 & 0
\end{array}\right]\left[\begin{array}{c}
\frac{\partial b}{\partial r} \\
b(r)
\end{array}\right]
$$

We initialise the system such that at the tip of the catheter is located in the close vicinity of the long axis of the coil $\left({ }_{\mathcal{T}}^{\mathcal{C}} \widehat{\mathbf{z}}\right)$. Hence, we assume that (6.11) holds approximately. We can then calculate the effective radius vector $\left(\mathbf{r}_{c} \in \mathbb{R}^{3}\right)$ as

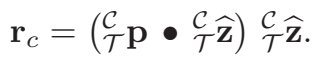


This vector is used to retrieve spherical coordinates $\left(\mathbf{s} \in \mathbb{R}^{3}\right)$ as shown in Figure 6.5. Furthermore, we calculate the offset $\left(\mathbf{o}_{c} \in \mathbb{R}^{3}\right)$ of the dipole centre from the coil axis $(\underset{\mathcal{T}}{\mathcal{C}} \widehat{\mathbf{z}})$ as follows:

$$
\mathbf{o}_{c}={ }_{\mathcal{T}}^{\mathcal{C}} \mathbf{p}+\mathbf{r}_{c}
$$

We use the retrieved parameters (6.15) and (6.16) to drive the inner loop of the system with the control law constraining the ARMM coil on a sphere centred at the location of catheter tip $\left({ }_{\mathcal{T}}^{\mathcal{C}} \mathbf{p}\right)$. Since the UR10 provides direct control over its joint velocities $\left(\dot{\mathbf{q}}_{m}\right)$, we use the inverse Jacobian controller

$$
\dot{\mathbf{q}}_{m}=\mathbf{J}_{t}^{-1}\left(\left[\begin{array}{c}
\mathbf{K}_{o}{ }_{\mathcal{C}}^{\mathcal{T}} \mathbf{R} \mathbf{o}_{c} \\
\mathbf{0}_{3 \times 1}
\end{array}\right]+\mathbf{K}_{e} \mathbf{J}_{e} \mathbf{e}_{s}\right),
$$

where $\mathbf{J}_{t} \in \mathbb{R}^{6 \times 6}$ is the UR10 end-effector Jacobian, $\mathbf{K}_{0}, \mathbf{K}_{e} \in \mathbb{R}^{3 \times 3}$ are the positive-definite gain matrices. The matrix $\left(\mathbf{J}_{e} \in \mathbb{R}^{6 \times 3}\right)$ defined as follows:

$$
\mathbf{J}_{e}=\left[\begin{array}{cccccc}
0 & 0 & -1 & 0 & 0 & 0 \\
-r \cos \theta & 0 & 0 & 0 & -\cos \phi & -\sin \phi \\
0 & r & 0 & -1 & 0 & 0
\end{array}\right]^{T}
$$

maps the spherical motion to velocity of the origin of frame $(\{\mathcal{T}\})$. The controller minimizes both the offset $\left(\mathbf{o}_{c}\right)$ as well as the error $\left(\mathbf{e}_{s}=\mathbf{s}_{\text {ref. }}-\mathbf{s}\right)$ between the reference and actual positions expressed in spherical coordinates.

The outer loop of the catheter controller provides reference values for the spherical parameters, the current of the ARMM coil and the position of the catheter inserter. We define a spherical actuation Jacobian $\left(\mathbf{J}_{s}\right) \in \mathbb{R}^{4 \times 6}$

$$
\mathbf{J}_{s}=\left[\begin{array}{ll}
\frac{\partial \mathbf{w}^{T}}{\partial \mathbf{s}} & \frac{\partial \mathbf{w}^{T}}{\partial I}
\end{array}\right]^{T} .
$$

This Jacobian is used along with the PRB model (6.9) to derive an equation describing the effect of all control inputs on the catheter tip velocity

$$
\mathbf{J}_{a} \mathbf{J}_{s}\left[\begin{array}{c}
\dot{\mathbf{s}} \\
\dot{I}
\end{array}\right]+\hat{\lambda} \dot{\lambda}=\mathbf{A}\left[\begin{array}{c}
\dot{\mathbf{s}} \\
\dot{I} \\
\dot{\lambda}
\end{array}\right]=\mathbf{A} \dot{\boldsymbol{\sigma}}={ }_{\mathcal{C}}^{\mathcal{G}} \dot{\mathbf{p}} .
$$

An analytic derivation of $\mathbf{J}_{s}$ is presented in the Appendix.

Inverting the actuation matrix $\left(\mathbf{A} \in \mathbf{R}^{3 \times 5}\right)$ allows us to compute the rate of reference control parameters $\left(\boldsymbol{\sigma}_{\text {ref. }} \in \mathbb{R}^{3} \times \mathbb{S}^{2}\right)$ which minimizes the catheter position error

$$
\dot{\boldsymbol{\sigma}}_{\text {ref. }}=\mathbf{A}^{\dagger}\left({ }_{\mathcal{C}}^{\mathcal{G}} \dot{\mathbf{p}}_{\text {ref. }}+\mathbf{K}_{c} \mathbf{e}_{c}\right)
$$

where $\dagger$ denotes weighted-damped pseudoinverse [220], $\mathbf{K}_{c} \in \mathbb{R}^{3 \times 3}$ is a positivedefinite gain matrix, and $\mathbf{e}_{c} \in \mathbb{R}^{3}$ is trajectory error is defined as follows:

$$
\mathbf{e}_{c}={ }_{\mathcal{C}}^{\mathcal{G}} \mathbf{p}_{\text {ref. }}-{ }_{\mathcal{C}}^{\mathcal{G}} \mathbf{p} \text {. }
$$


The heuristically established weights in the weighted-damped pseudoinverse improve the behaviour of the resulting solution. In our case, they are predominantly used to limit the displacement rates along $r$ to avoid the collisions of the mobile coil. Additionally, this technique inherently allows imposing limits on control variables, avoiding collisions with a predefined workspace.

\subsubsection{Stage II: Projectile Targeting}

Once the catheter is delivered close to the target site, the second stage of the drug delivery procedure is realised. In this stage, the capsule with the active substance is released from the catheter by reversing the current in the coil located on the catheter tip. It moves to the final target as a projectile under the influence of the magnetic wrench $\left(\mathbf{w}_{\mu}\right)$. By modifying this wrench, the trajectory of that projectile can be guided remotely towards the target of choice.

However, due to the absence of feedback modality capable of continuously tracking the position of the projectile, this guidance cannot effectively be realised in a closed-loop fashion. Overcoming that problem, we propose an alternative approach, in which the trajectory of the projectile is determined before ejection. Our approach exploits the advantage of actuation with a single mobile electromagnet (Figure 6.7). Magnetic wrench $\left(\mathbf{w}_{\mu}\right)$ experienced by the projectile in external magnetic field generated by such a device stabilises its trajectory. If ejected close to the long axis $\left({ }_{\mathcal{T}}^{\mathcal{C}} \widehat{\mathbf{z}}\right)$ the projectile eventually converges to follow that axis regardless of its initial pose. Thus, by changing the direction of ${ }_{\mathcal{T}}^{\mathcal{C}} \widehat{\mathbf{z}}$ before the projectile release, we can modify the trajectory, such that the projectile reaches the target with no closed-loop guidance.

We exploit the over-actuation of the principal catheter controller (6.21) to develop a targeting controller. We define the error between the target $(t)$ and the actual $(a)$ orientation of the long axis of the mobile electromagnet, expressing it using spherical angles $(\theta, \phi)$ as follows:

$$
\mathbf{e}_{r}=\left[\begin{array}{ll}
e_{\theta} & e_{\phi}
\end{array}\right]^{T}=\left[\begin{array}{ll}
\theta_{\mathrm{t}}-\theta_{\mathrm{a}} & \phi_{\mathrm{t}}-\phi_{\mathrm{a}}
\end{array}\right]^{T} .
$$

We perform the targeting of the projectile by minimizing this error with motion within the null-space of the system. This way, we reconfigure the ARMM system, while keeping the position of the catheter tip steady. We realise the targeting iteratively, using a resolved-rate approach, adding the resulting control input to the position controller

The controller utilises information about the configuration of the system as provided by the scanning ultrasound technique. We use the LU decomposition, to compute the kernel

$$
\operatorname{ker}(\mathbf{A})=\left[\begin{array}{ll}
\boldsymbol{\nu}_{\theta} & \boldsymbol{\nu}_{\phi}
\end{array}\right] \in \mathbb{R}^{5 \times 2}
$$

where

$$
\begin{aligned}
\boldsymbol{\nu}_{\theta} & =\left[\begin{array}{lllll}
\nu_{\theta}^{r} & 1 & 0 & \nu_{\theta}^{I} & \nu_{\theta}^{\lambda}
\end{array}\right]^{T}, \\
\boldsymbol{\nu}_{\phi} & =\left[\begin{array}{lllll}
\nu_{\phi}^{r} & 0 & 1 & \nu_{\phi}^{I} & \nu_{\phi}^{\lambda}
\end{array}\right]^{T},
\end{aligned}
$$




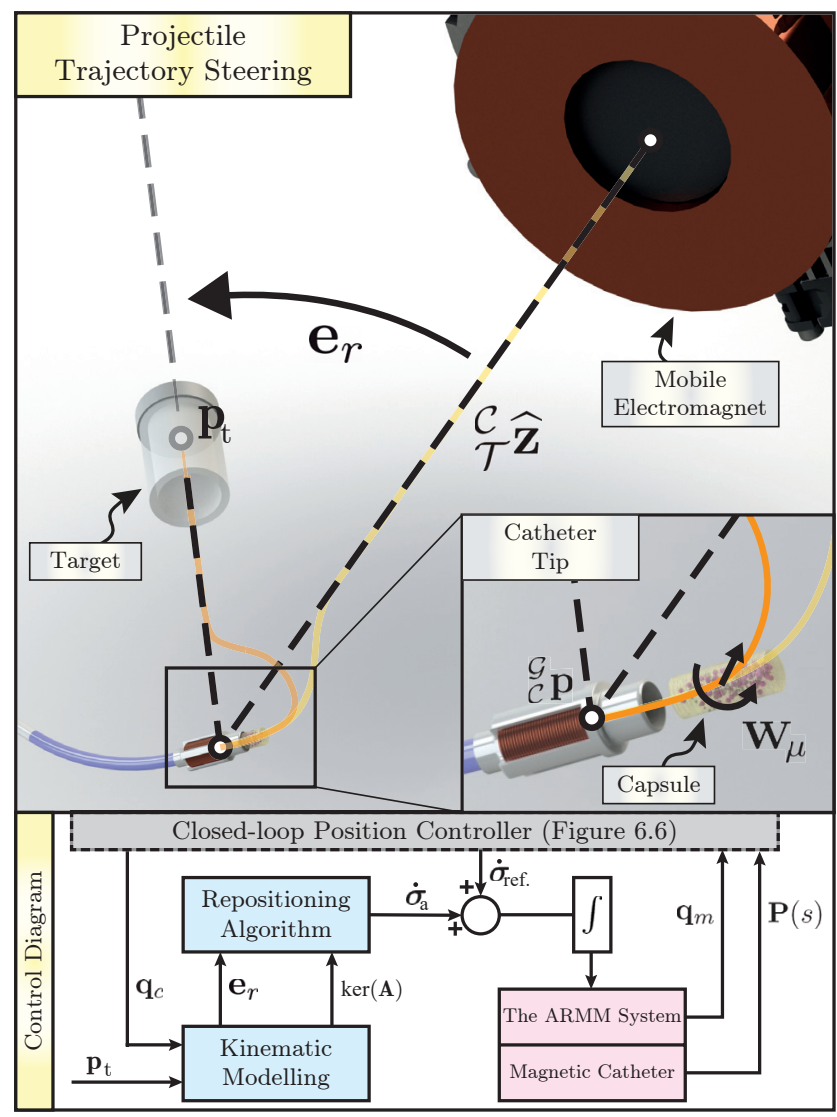

Figure 6.7: After ejection from the dock at the tip of the catheter, the capsule moves as a projectile. This projectile converges to the trajectory along the long axis $\left(\mathcal{C}_{\mathcal{T}}^{\mathcal{Z}} \in \mathbb{R}^{3}\right)$ of the mobile electromagnet, determined by its configuration $\left(\mathbf{q}_{m} \in \mathbb{R}^{3}\right)$. This happens due to the effect of the same magnetic wrench $\left(\mathbf{w}_{\mu} \in \mathbb{R}^{6}\right)$ generated by the Advanced Robotics for Magnetic Manipulation (ARMM) system, which is used for controlling the shape $(\mathbf{P}(s))$ of the catheter. We use that property in a repositioning control scheme, to target the projectile at a target site located at $\left(\mathbf{p}_{t} \in \mathbb{R}^{3}\right)$. The scheme iteratively minimizes the error $\left(\mathbf{e}_{r} \in \mathbb{S}^{2}\right)$ between the current and desired electromagnet orientation as expressed in the coordinates of the spherical constraint. The control inputs $\left(\dot{\boldsymbol{\sigma}}_{\mathrm{a}} \in \mathbb{R}^{3} \times \mathbb{S}^{2}\right)$ are computed using the kernel $\left(\operatorname{ker}(\mathbf{A}) \in \mathbb{R}^{5 \times 2}\right)$ and added to the reference $\left(\dot{\boldsymbol{\sigma}}_{\text {ref. }} \in \mathbb{R}^{3} \times \mathbb{S}^{2}\right)$ from the closed-loop position controller (Figure 6.6) Since $\operatorname{ker}(\mathbf{A})$ describes the span of the actuation null-space, the targeting occurs with no motion of the catheter tip located at $\left({ }_{\mathcal{C}}^{\mathcal{G}} \mathbf{p} \in \mathbb{R}^{3}\right)$.

are the vectors describing the span of the null-space of the system (6.20). The rates of change within the null-space do not generate the motion of the catheter tip.

Therefore, we can use the vectors within the kernel $(k e r(\mathbf{A}))$ to reconfigure the long axis of the mobile electromagnet, while keeping the catheter steady. For each iteration of our targeting controller, we can calculate the rate of spherical 
parameters minimizing the error (6.23) as follows:

$$
\dot{\boldsymbol{\sigma}}_{\mathrm{a}}=K_{a}\left(e_{\theta} \boldsymbol{\nu}_{\theta}+e_{\phi} \boldsymbol{\nu}_{\phi}\right) \text {. }
$$

This rate is added to the reference from (6.21) and fed to the low-level spherical constraint controller (Figure 6.7), until the expected trajectory of the projectile is considered sufficiently close to the reference. Subsequently, the projectile is ejected from the tip and moves in open-loop conditions.

\subsection{Experimental Setup}

We test our catheter experimentally in a dedicated test-bed shown in Figure 6.8. The catheter is inserted into a PMMA tank filled with water, using LX15 linear actuator (Misumi Europa GmbH, Schwalbach am Taunus, Germany). The ultrasound scanner presented in Section 6.3 is mounted at the side of the tank, imaging the water-filled volume through a silicon membrane. The scanner is motorized using a LX20 linear actuator (Misumi Europa GmbH). The linear actuators of both the inserter and the scanner are position-controlled using iPOS4808 BX-CAT drives (Technosoft Motion).

The poses of all components of the test-bed are registered within the workspace of the ARMM system. The tracking and control framework presented in previous sections is implemented in $\mathrm{C}++$ on Linux Ubuntu 16.04 workstation (Kernel 4.4, Intel Xeon E5 CPU, NVidia Quadro K4200 GPU and 32 GB RAM). The inner spherical constraint controller (Figure 6.6) is timed at $125 \mathrm{~Hz}$, which is the internal rate used by the UR10. The rate of the inverse model controllers (6.21) and (6.27) is limited by the speed of the ultrasound scanner. The system requires approximately $7 \mathrm{~s}$ for a single scan.

\subsection{Results}

The experimental setup is used to test our magnetic catheter (Figure 6.2). Since the scanning ultrasound is the principal tracking method used for shape reconstruction, we start by validating its accuracy. Subsequently, we demonstrate Stage I of our method. We present the experiments involving closed-loop position control of magnetic catheter across a series of trajectories using the inverse model controller. The data from these experiments are then used in a computational study investigating the capabilities of the targeting algorithm (Figure 6.7). Finally, we execute a proof-of-concept projectile delivery experiment, demonstrating the feasibility of our approach.

\subsubsection{Validation of Catheter Shape Reconstruction Using Scanning Ultrasound}

We quantify the accuracy of our shape reconstruction method using two mock-ups with known shapes as a ground truth (Figure 6.9). The mock-ups are designed using SolidWorks 2018 CAD (Dassault Systèmes, Vélizy-Villacoublay, France) and printed using Form2 SLA 3D printer (Formlabs Inc., Somerville, USA). Subsequently, they are fixed within the test-bed, with their poses registered with respect 


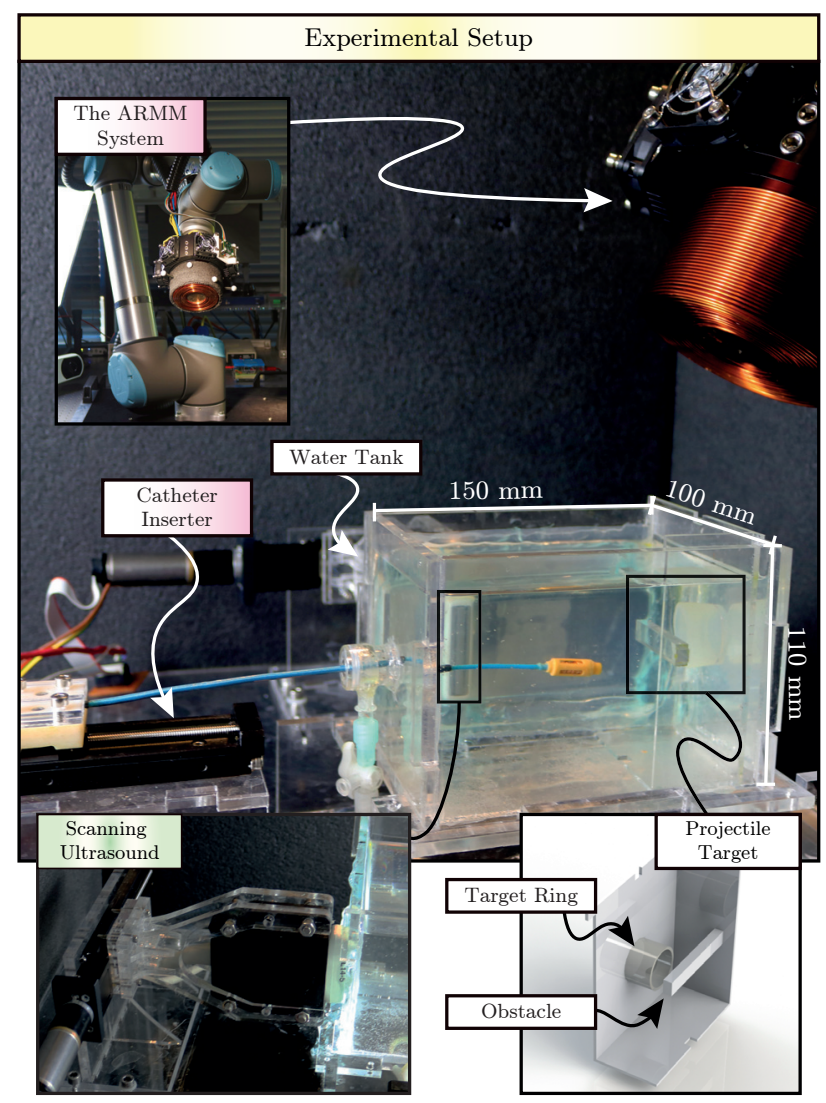

Figure 6.8: The test-bed used for experimental studies involving our magnetic catheter. The Advanced Robotics for Magnetic Manipulation (ARMM) system is used to control the catheter in a water-filled tank. The catheter is inserted into the tank using an LX15 linear actuator (Misumi Europa GmbH, Schwalbach am Taunus, Germany). During the experiments, the catheter is tracked using the scanning ultrasound technique (Figure 6.4) through an ultrasound-transparent wall made out of silicon rubber. For experiments involving projectile delivery, a Poly(Methyl Methacrylate) (PMMA) target is fabricated and placed at the far end of the tank. The target contains a Polyvinyl chloride (PVC) ring, into which the projectile has to be delivered, and a horizontal obstacle positioned in front of the ring.

to the frame $\{\mathcal{G}\}\}$. The ultrasound scanner is used to acquire 5 independent stacks of images representing each mock-up.

Each image stack is processed independently by our shape reconstruction algorithm (Figure 6.4) to obtain a polynomial representation $(\mathbf{P}(s))$. Subsequently, the CAD models are converted into a set of points along the centreline of each mock-up. These points are used to fit the ground truth polynomials. The ground truth is compared with the reconstruction by using the respective polynomials to generate sets of $1003 \mathrm{D}$ points at corresponding lengths $(s)$.

The results (Figure 6.9) show good correspondence of the reconstructed shapes with the ground truth with the mean errors of $0.37 \mathrm{~mm}$ and $0.63 \mathrm{~mm}$. We additionally quantify the standard deviations of the errors, which are $0.24 \mathrm{~mm}$ and $0.58 \mathrm{~mm}$ for mock-up 1 and 2 respectively. The discrepancies between the indi- 


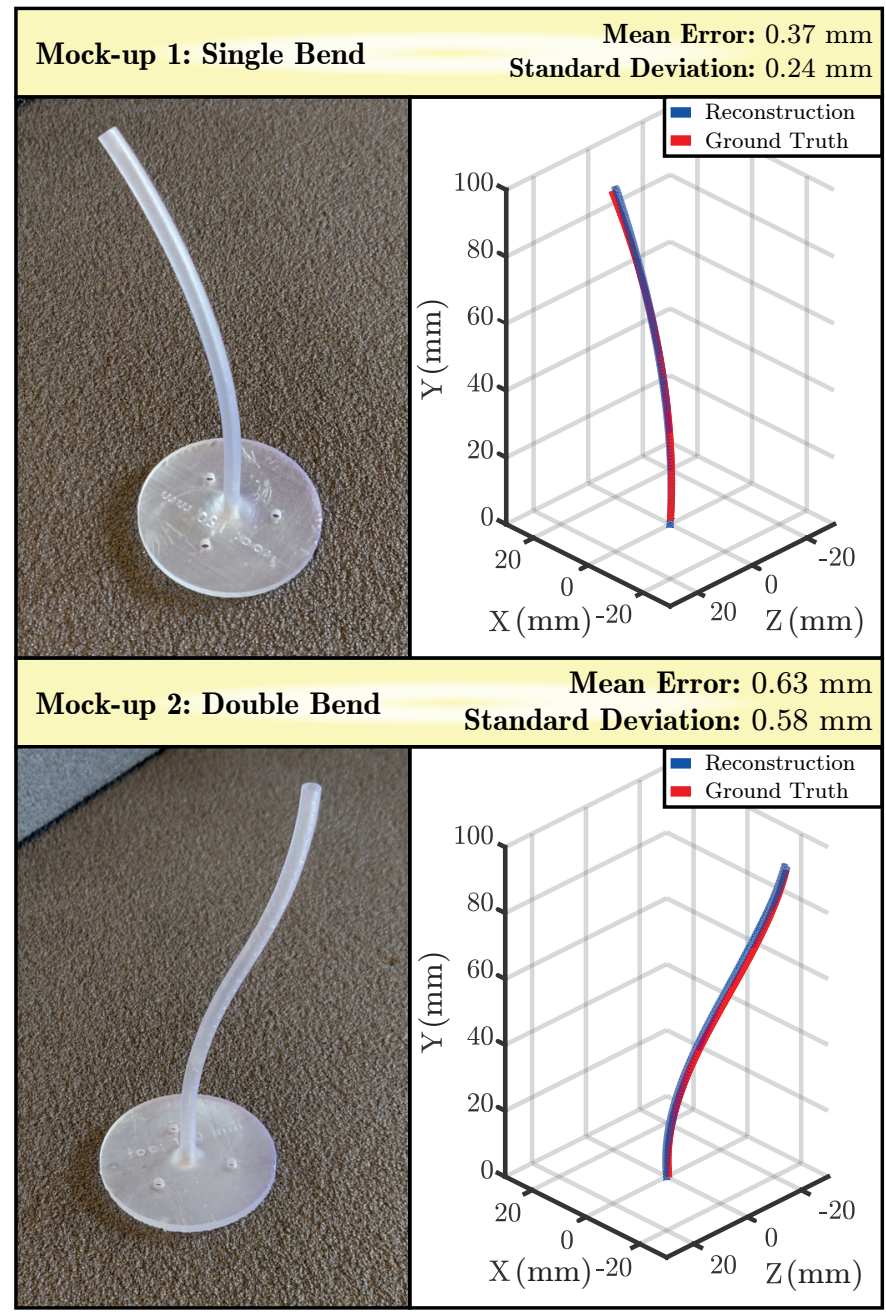

Figure 6.9: The accuracy of the catheter tracking using scanning ultrasound technique is established using two mock-ups with predefined shapes as a ground truth. The mock-ups (Left) are $3 \mathrm{D}$ printed and fixed in a known pose within the experimental test-bed. The ultrasound scanner is used to collect 5 independent stacks of B-mode images corresponding to each mock-up. Each stack is processed using our shape reconstruction algorithm to recover a polynomial model. The same polynomial model is fitted onto a set of points along the centreline of each mock-up, generating ground truth. The error metrics are generated by comparing the positions (Right) of 100 points at corresponding lengths along the continuous shape. The errors between the shapes reconstructed from individual stacks are negligible (below $0.1 \mathrm{~mm}$ ).

vidual scanning stacks are negligible (below $0.1 \mathrm{~mm}$ ). This allows us to conclude that the scanning ultrasound can be used reliably as a tracking method for our magnetic catheter. 


\subsubsection{Closed-loop Position Control of Magnetic Catheter}

The inverse-model position controller (Figure 6.6) is tested experimentally in a study involving steering of our magnetic catheter through a series of trajectories. The trajectories are defined using point-to-point parabolic segments. The trajectories are selected arbitrarily to cover the entire possible range of catheter deflections, both when the catheter is deflected both towards (positive current) and away from (negative current) ARMM coil. Before each trajectory, a volumetric ultrasound scan is taken to align the ARMM system in a predefined position in spherical coordinates (s). The results of the study are shown in the Figure 6.10. The minimum reported mean error is $0.82 \mathrm{~mm}$ for Trajectory 2, whereas Trajectory 1 was executed with the smallest maximum error norm of $2.66 \mathrm{~mm}$.

\subsubsection{Projectile Targetting: Computational Analysis}

With respect to the targeting algorithm (Figure 6.7), an important question concerns the limits of possible reconfiguration given a particular state of the system. In the context of highly complex mechanics of the catheter actuated by the ARMM system it remains unlikely that the transition between any two orientations of the long axis $\left({ }_{\mathcal{T}}^{\mathcal{C}} \widehat{\mathbf{z}} \in \mathbb{R}^{3}\right)$ is possible within the practical bounds of the control input $(\boldsymbol{\sigma})$. This directly impacts the effective reach of a projectile delivered using our method from a given location. The analysis of this reach is therefore vital to understanding the capabilities of our targeting method.

We proceed by using the data collected in the closed-loop control study. For every configuration across the three trajectories, we calculate the kernel $(\operatorname{ker}(\mathbf{A}))$ to analyse the span of the corresponding null-space. In each case the span can be converted to the form described by (6.25) and (6.26), which indicates that for every configuration of the catheter assumed during the steering experiment, the targeting is possible along both $\theta$ and $\phi$. However, by comparing the average ratio of the two constituent vectors of the kernel for all configurations, we determine that $\left(\left\|\boldsymbol{\nu}_{\theta}^{\prime}\right\|=\left\|\left[\begin{array}{lll}\nu_{\theta}^{r} & \nu_{\theta}^{I} & \nu_{\theta}^{\lambda}\end{array}\right]^{T}\right\|\right)$ is on average 228 times higher than $\left(\left\|\nu_{\phi}^{\prime}\right\|=\left\|\left[\begin{array}{lll}\nu_{\phi}^{r} & \nu_{\phi}^{I} & \nu_{\phi}^{\lambda}\end{array}\right]^{T}\right\|\right)$. This allows us to confer that the reconfiguration along $\phi$ results in significantly less change in system variables and hence it is easier to achieve given the bounds of the system.

We further investigate this phenomenon by numerically finding the optimal combination of rotations $\left(\left[\begin{array}{ll}\hat{\theta}_{o} & \hat{\phi}_{o}\end{array}\right]\right)$ such that

$$
\hat{\theta}_{o}^{2}+\hat{\phi}_{o}^{2}=1
$$

This combination determines the direction, which for each state of the catheter allows for null-space targeting with the minimal change of system parameters $(\boldsymbol{\sigma})$. We do this by numerically solving the optimization problem as follows:

$$
\hat{\theta}_{o}=\underset{\hat{\theta} \in(-1,1)}{\operatorname{argmin}}\left(\left\|\boldsymbol{\nu}_{\theta}^{\prime} \hat{\theta} \pm \boldsymbol{\nu}_{\phi}^{\prime} \sqrt{\left(1-\hat{\theta}^{2}\right)}\right\|\right)
$$




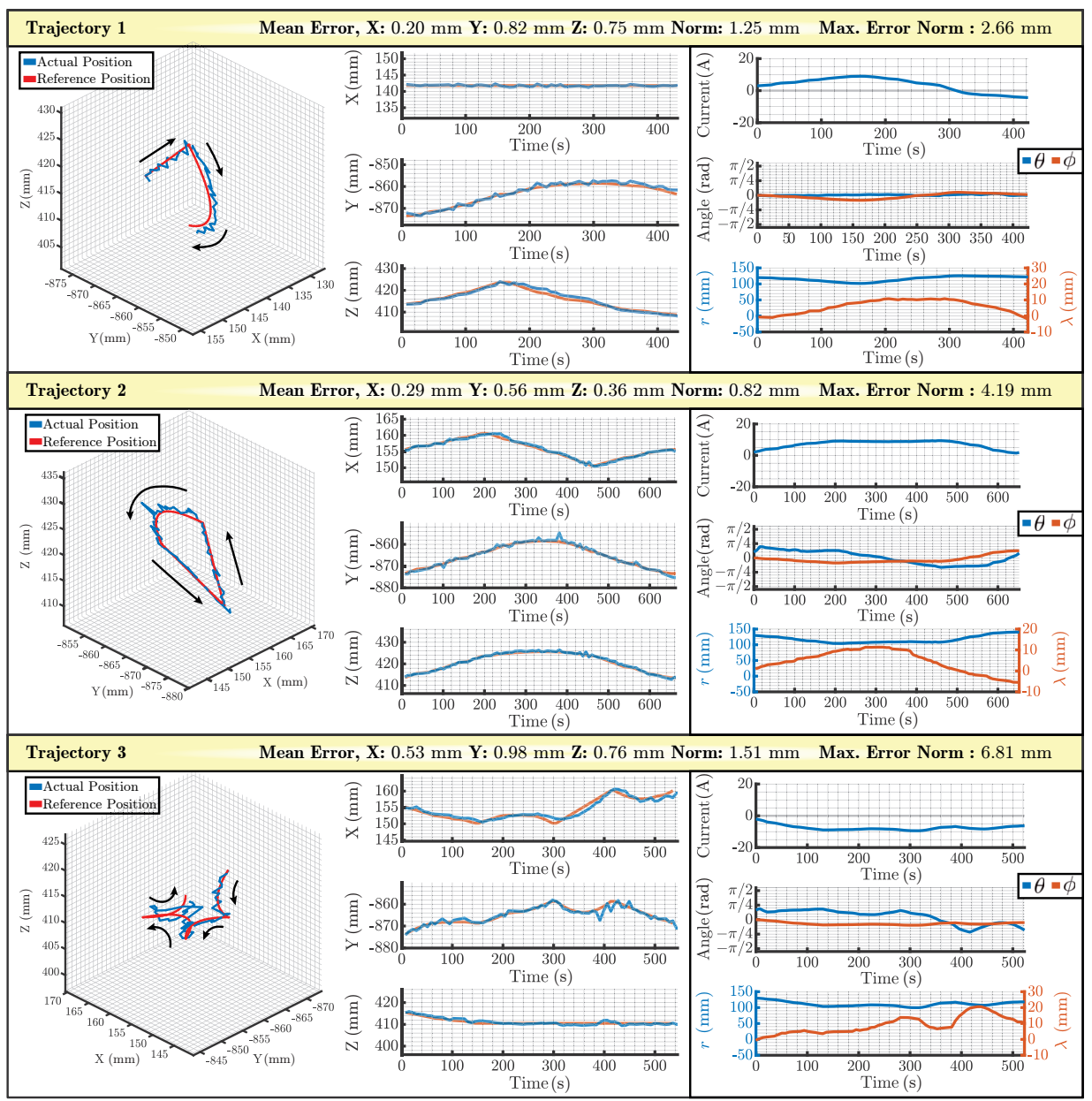

Figure 6.10: The closed-loop position controller is tested in an experiment involving steering of the magnetic catheter across three predefined trajectories. Left: The trajectory of catheter tip in carthesian coordinates $(X, Y, Z)$ of global reference frame $(\{\mathcal{G}\})$. Centre: The time plots of reference and actual tip position for each Cartesian coordinate. Right: The time plots of the control inputs used for catheter steering: the current in the mobile electromagnet $(I \in \mathbb{R})$, the parameters $\left(\mathbf{s}=\left[\begin{array}{lll}r & \theta & \phi\end{array}\right]^{T} \in \mathbb{R}^{+} \times \mathbb{S}^{2}\right)$ of the spherical constraint (Figure 6.5) and the position $(\lambda \in \mathbb{R})$ of the motorized catheter inserter.

for every state of the system recorded during the closed-loop trajectories (Figure 6.10). Subsequently, we compute the corresponding $\hat{\phi}_{o}$ from (6.28). For each state of the system two minima exist, which are symmetric around the 0 point. The solution of our study indicates that in each case the optimal reconfiguration direction is very close to $\phi$, with the mean $\left|\hat{\phi}_{o}\right|$ of 0.98 and mean $\left|\hat{\theta}_{o}\right|$ of 0.043 .

\subsubsection{Projectile Delivery: Experiments}

We use the results of the computational analysis to determine the conditions for our projectile targeting experiment. Since more in-depth work on trajectory planning 
is beyond the scope of this chapter, we decide to confine the targeting experiment to a vertical plane. This way we can demonstrate the performance of our magnetic catheter in projectile delivery, while leveraging on the straightforward relation between the target location and the position of the catheter tip for successful reconfiguration within the bounds of the system.

The results of our experiment are presented in Figure 6.11. After bringing the catheter into the final configuration, we use the targeting algorithm (6.27) to reconfigure the mobile electromagnet such that its long axis is brought into the vicinity of the target. Throughout the reconfiguration, we report the mean catheter tip displacement of $0.88 \mathrm{~mm}$. Due to gravitational force having a large impact on the motion of the projectile, we compensate for it by offsetting the reference angular position $\left(\phi_{t}\right)$ by $20^{\circ}$. Once the targeting finishes, the projectile is ejected from the catheter tip and successfully reaches the target. Subsequently, we realise the retrieval of the projectile by repositioning an inactive mobile electromagnet. Upon activation of the electromagnet, the projectile is drawn back to the catheter tip, which allows for its extraction.

Additionally, we execute a second trial to study the motion of the projectile upon ejection. For that purpose, we use a MotionBlitz EoSens ${ }^{\circledR}$ Cube7 high-speed camera (Mikrotron GmbH, Unterschleißheim, Germany) to record a trajectory at 1000 FPS. The results of that experiment are presented in Figure 6.12. The projectile successfully reaches the target within $200 \mathrm{~ms}$ and its trajectory is consistent with the expected behaviour shown in Figure 6.7.

\subsection{Discussion}

The experimental work presented in this chapter demonstrates that a magnetic catheter can successfully be used as a delivery vehicle for untethered agents, such as our capsule for targeted drug delivery. By using external magnetic fields provided by a mobile electromagnet, the catheter can be steered into the vicinity of the target. Subsequently, the catheter can eject the capsule, which is delivered to the target as a projectile. Overacuation of the system can be used to determine the final trajectory of the projectile, effectively allowing to aim at the target.

The principal factor, which restricts the performance of our system at all stages of the delivery procedure, is a very slow tracking rate of the scanning ultrasound. As we rely only on this modality for state feedback, the effective rate of our controllers is approximately $0.14 \mathrm{~Hz}$. This results in control performance effectively worse than in comparable previous work involving high-speed optical tracking (which is nevertheless not clinically relevant) [116, 194]. Furthermore, such a low control rate has a negative impact on the stability of the system. This predominantly affects the ability of the inner control loop to enforce spherical constraint at larger deflections of the catheter, where significant magnetic forces are present.

There are several ways in which the shape reconstruction rate could be improved. First of all, as this rate is determined by the speed of the mechanical displacement of the ultrasound transducer, which could be increased by using faster linear actuation unit. This, however, would result in significant increase in shear at the contact surface. If the resulting tracking rate was to be increased to values comparable with camera-based modalities, the technique would become unsuit- 


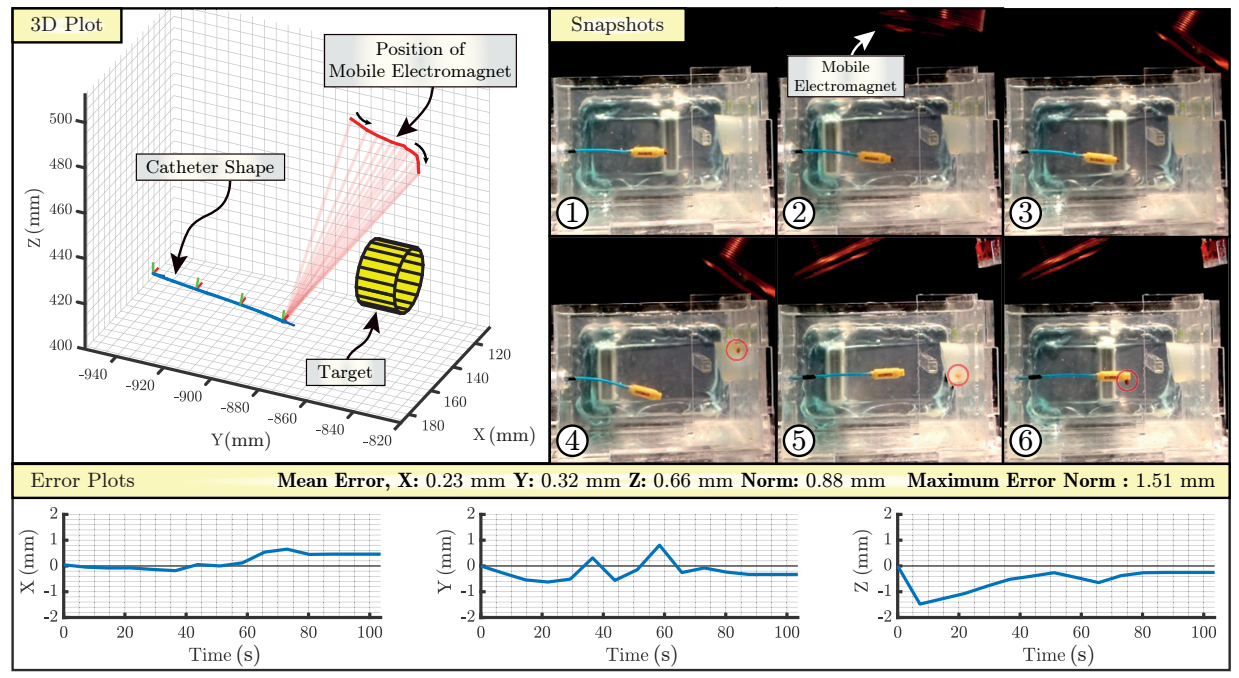

Figure 6.11: The targeting algorithm is used to deliver the projectile to a target within the workspace. Left: the 3D plot of the position of the mobile electromagnet during reconfiguration. Right: snapshots showing consecutive stages of the delivery procedure. (1) The catheter is located within the workspace with no actuation. (2) The mobile electromagnet is used to deflect it to the ejection position. (3) The targeting algorithm is used to change the pose of the mobile electromagnet. (4) The projectile (red circle) is ejected and reaches the target. (5) The mobile electromagnet is reconfigured for projectile retrieval. (6) The mobile electromagnet is activated and the retrieved projectile is driven towards the catheter. Bottom: the error plots showing the displacement of the catheter from the ejection position during the reconfiguration if the mobile electromagnet.

able for clinical use. Alternative, clinically-relevant methods could involve using three-dimensional ultrasound transducers or Fiber-Bragg Grating (FBG) based proprioceptive shape sensing [166,221,222]. An ultrasound-FBG sensor fusion method can also be considered for improved robustness [223]. Finally, multi-rate state observers could be used to combine estimation based methods and the scanning ultrasound for improved effective feedback rate [218, 224].

Other aspects, where improvements can be made regarding the catheter control, involve replacing the spherical constraint actuation model, with a more complex framework. The spherical constraint is burdened with inherent singularities, which occur when the coil current $(I)$ is zero and when $(\phi)$ reaches $90^{\circ}$. Throughout the experiments, we observed that by the use of damping in our inverse model, the current singularity poses no problem to the performance of the system, as demonstrated in Trajectory 1 (Figure 6.10). Nevertheless, the use of advanced, polynomial field models could potentially alleviate the problem completely, additionally allowing us to use the mobile electromagnet regardless of its orientation with respect to the catheter tip [96]. Finally, the magnetic actuation system could be expanded to involve several independent mobile coils, which would vastly enhance the control capabilities of the system [225].

Regrading the projectile delivery, our work demonstrates the potential of this approach for targeted drug delivery and other medical procedures performed using 


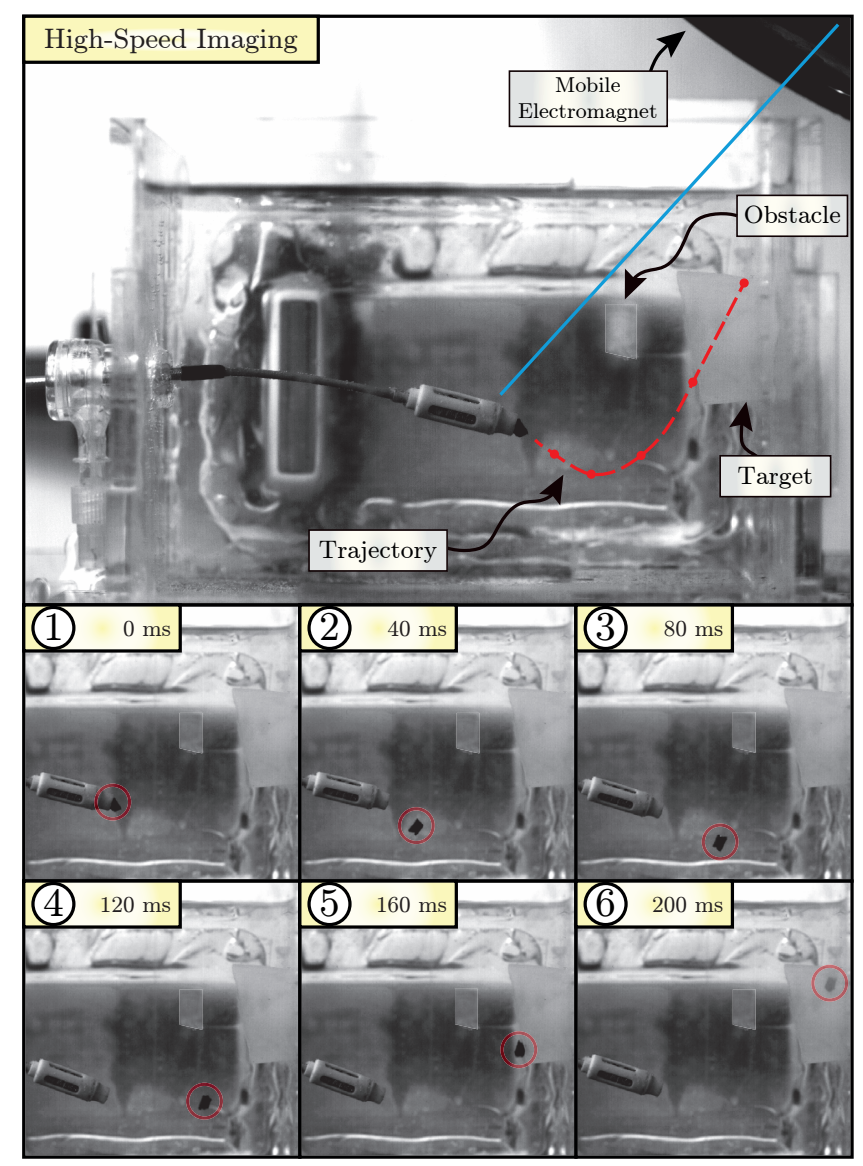

Figure 6.12: We study the trajectory (red line) of the projectile (red circle) by using a MotionBlitz EoSens ${ }^{\circledR}$ Cube7 high-speed camera (Mikrotron GmbH, Unterschleißheim, Germany) to register its motion (1) - 6) upon ejection from the catheter. We image the workspace at 1000 FPS. The results indicate that the projectile follows a trajectory coherent with the assumption made by the proposed targeting algorithm (Figure 6.7). However, the combined effects of gravity and initial acceleration due to ejection force, substantially increase the distance at which the projectile converges with the long axis of the mobile electromagnet (blue), thus degrading the final targeting accuracy.

milli/microrobotic untethered agents. However, the scope of our work naturally requires expansion before being translated into clinical practice. Improvements are required regarding both the design of untethered agents to be delivered by magnetic catheters as well as the techniques for steering them towards a selected target.

The capsules used in our work can effectively carry an active substance contained within a biodegradable matrix to a given location. Nevertheless, demonstration of the controlled release of a given substance, as well as its effect in a clinically-relevant environment remain outside of the scope of this chapter. Therefore, the concept needs to be further elaborated, creating fully bioactive designs capable of localized action. 
By using permanent magnets within the capsule, we ensure both adequate magnetic properties, as well as containment of the potentially harmful rare-earth alloy within a solid, easily-retrievable structure. Nevertheless, our approach has its drawbacks, as the resulting weight of the capsule is large, pronouncing the effects of gravity. Recent progress in development of soft magnetic materials may provide an alternative approach, whereby magnetic particles could be incorporated directly within the biocomposite material $[148,226]$. However, the resulting designs must either allow for easy retrieval of the degraded debris, or ensure the biocompatibility of its hard magnetic phase.

The targeting of an untethered agent moving as a projectile should also be a subject to further study. The trajectory of the projectile is determined by the nature of the external magnetic field, and thus on a particular magnetic actuation system. For a single mobile electromagnet, such as the ARMM system, targeting is possible exploiting the overactuation of the catheter. Nevertheless, it remains challenging without a reliable path planning technique. Thus, we are unable to determine whether, for a given catheter location, successful trajectories are attainable within the bounds of the actuation system. Alternatively, the use of multiple electromagnets could potentially alleviate the problem, expanding the range of targets reachable from a particular location, although such actuation systems would potentially require a different targeting strategy due to more complex magnetic field patterns they generate.

Additionally, a dynamic model of a projectile could help understanding its behaviour and predicting the resulting trajectories. As experienced in our experiment, gravity and the forces experienced during the ejection play a significant role in determining the motion of the projectile, therefore degrading the targeting accuracy. Models taking into account these phenomena could in the future be incorporated into more advanced targeting algorithms, enabling precise targeting also in clinically-relevant conditions.

\subsection{Conclusions}

In this chapter we present a magnetic catheter capable for controller release and retrieval of untethered magnetic capsules. We propose to use our catheter in an ultrasound-guided targeted drug delivery procedure, whereby the magnetic capsule is released from the catheter and navigated into the target as a projectile. We use the mobile electromagnet of the Advanced Robotics for Magnetic Manipulation (ARMM) system as a source of the external field for magnetic actuation. In our procedure, the catheter is steered into the vicinity of the target with a closed-loop position controller under the guidance of volumetric scanning ultrasound. Upon reaching the final position of the catheter tip, a targeting algorithm is used to reconfigure the pose of the mobile electromagnet, which determines the trajectory of the projectile, subsequently ejected from the catheter.

The catheter is tested in a series of experiments. We first determine the accuracy of the catheter shape reconstruction using scanning ultrasound, reporting the best mean error of $0.37 \mathrm{~mm}$. Subsequently, we use our closed-loop controller to guide the catheter along a set of trajectories with the best error of $0.82 \mathrm{~mm}$. Finally, we realise a projectile delivery and retrieval, demonstrating successful use 
of our targeting algorithm to reach a designated target with a mean catheter tip displacement of $0.88 \mathrm{~mm}$.

\section{Appendix}

The spherical actuation Jacobian $\left(\mathbf{J}_{s}\right)$ is defined as follows:

$$
\left(\mathbf{J}_{s}\right)=\left[\begin{array}{llll}
\frac{\partial \mathbf{w}}{\partial r} & \frac{\partial \mathbf{w}}{\partial \theta} & \frac{\partial \mathbf{w}}{\partial \phi} & \frac{\partial \mathbf{w}}{\partial I}
\end{array}\right]
$$

and is derived from the model (6.14). The field $(b(r, I))$ and gradient maps within that model were presented in Chapter 3 . They have the non-linear form

$$
\begin{aligned}
b(r, I) & =\left(b_{w}(r)+\eta(I) b_{c}(r)\right) \\
\frac{\partial b}{\partial r} & =\left(\frac{\partial b_{w}}{\partial r}+\eta(I) \frac{\partial b_{c}}{\partial r}\right),
\end{aligned}
$$

where $b_{w}(r), b_{c}(r): \mathbb{R}^{+} \mapsto \mathbb{R}^{+}$are unit field maps for the windings and the core of the mobile electromagnet respectively. The coefficient $(\eta(I) \in(0,1])$ is used to take into account the saturation of electromagnet core, which happens above \pm 15 A. Combining (6.14) with (6.31) and (6.32), we can analytically derive the individual columns of (6.30) as follows:

$$
\frac{\partial \mathbf{w}}{\partial r}=I \mu\left[\begin{array}{c}
-\frac{3}{2} \sin \theta \sin \phi \cos \phi \frac{\partial^{2} b}{\partial r^{2}} \\
\frac{3}{2} \cos \theta \sin \phi \cos \phi \frac{\partial^{2} b}{\partial r^{2}} \\
\left(\sin ^{2} \phi-0.5 \cos ^{2} \phi\right) \frac{\partial^{2} b}{\partial r^{2}} \\
-\cos \theta \cos \phi \frac{\partial b}{\partial r} \\
-\sin \theta \cos \phi \frac{\partial b}{\partial r} \\
0 \\
\frac{\partial \mathbf{w}}{\partial \theta}=I \mu\left[\begin{array}{c}
-\frac{3}{2} \cos \theta \sin \phi \cos \phi \frac{\partial b}{\partial r} \\
-\frac{3}{2} \sin \theta \sin \phi \cos \phi \frac{\partial b}{\partial r} \\
0 \\
\sin \theta \cos \phi b(r, I) \\
-\cos \theta \cos \phi b(r, I) \\
0
\end{array}\right]
\end{array}\right.
$$




$$
\frac{\partial \mathbf{w}}{\partial \phi}=I \mu\left[\begin{array}{c}
\frac{3}{2} \sin \theta\left(\cos ^{2} \phi-\sin ^{2} \phi\right) \frac{\partial b}{\partial r} \\
\frac{3}{2} \cos \theta\left(\cos ^{2} \phi-\sin ^{2} \phi\right) \frac{\partial b}{\partial r} \\
3 \sin \phi \cos \phi \frac{\partial b}{\partial r} \\
\cos \theta \sin \phi b(r, I) \\
\sin \theta \sin \phi b(r, I) \\
0
\end{array}\right]
$$

$$
\frac{\partial \mathbf{w}}{\partial I}=\mu\left[\begin{array}{c}
-\frac{3}{2} \sin \theta \sin \phi \cos \phi\left(\frac{\partial b}{\partial r}+I \frac{\partial b_{c}}{\partial r} \frac{\partial \eta}{\partial I}\right) \\
\frac{3}{2} \cos \theta \sin \phi \cos \phi\left(\frac{\partial b}{\partial r}+I \frac{\partial b_{c}}{\partial r} \frac{\partial \eta}{\partial I}\right) \\
\left(\sin ^{2} \phi-0.5 \cos ^{2} \phi\right)\left(\frac{\partial b}{\partial r}+I \frac{\partial b_{c}}{\partial r} \frac{\partial \eta}{\partial I}\right) \\
-\cos \theta \cos \phi\left(b(r, I)+I b_{c} \frac{\partial \eta}{\partial I}\right) \\
-\sin \theta \cos \phi\left(b(r, I)+I b_{c} \frac{\partial \eta}{\partial I}\right) \\
0
\end{array}\right] .
$$





\section{Part III}

\section{A Matter of Perspective: Catheters as Magnetic Actuation Systems}





\section{Preface}

The previous parts of this dissertation considered magnetic catheters as robotic tools, used for execution of tasks inside the human body. Prototypes discussed within Part 2 demonstrated how increasingly sophisticated magnetic end-effectors can expand the behaviour of a flexible catheter. In particular, the use of miniaturized electromagnets in the designs allowed for creation of catheters, which rely on internal mechatronic components for at least some part of their behaviour. Both in Chapters 5 and 6, by controlling the current in miniaturized electromagnets, a catheter is used to exert magnetic wrenches on parts of itself: the tip magnet and the capsule respectively. To a degree, such devices take part in the task intended originally for BigMag and the ARMM system. Catheters become magnetic actuation systems, although at much smaller scales.

We bring this concept further, rethinking classical systems for magnetic actuation of untethered microagents. These agents, with various form and function, are usually manipulated using macroscale systems comparable to BigMag or the ARMM system. In Chapter 7, we show that similar tasks can be realised by a mesoscale catheter with significantly reduced footprint. The result of that study - MILiMAC (Microrobotic Infrastructure Loaded into MagneticallyActuated Catheter) - is a flexible catheter with three parallel miniaturized electromagnets. MILiMAC can be deployed in deeply-seated regions of human body, creating a workspace for two-dimensional control of microrobotic agents. The agents are actuated by magnetic wrenches, generated by controlling the current in each miniaturized electromagnet. In this study we test MILiMAC in a proof-ofconcept experiment, in which the catheter is inserted into a test bed and deployed around a target site. Subsequently, a ferromagnetic microbead is delivered into that workspace and steered along point-to-point trajectories under feedback from optical camera. 



\title{
Chapter 7
}

\section{MILiMAC: A Flexible Probe for Delivery and On-site Magnetic Control of Medical Microrobotic Agents}

\begin{abstract}
Advancements in medical microrobotics have given rise to an abundance of agents capable of localised interaction with human body in small scales. Nevertheless, clinically-relevant applications of this technology are still limited by the auxiliary infrastructure required for actuation of micro-agents. In this chapter, we approach this challenge. Using finite-element analysis, we show that miniaturization of electromagnets can be used to create systems capable of providing magnetic forces adequate for micro-agent steering, while retaining small footprint and power consumption. We use these observations to create MILiMAC (Microrobotic Infrastructure Loaded into Magnetically-Actuated Catheter). MILiMAC is a flexible catheter employing three miniaturized electromagnets to provide localized magnetic actuation at the deeply-seated microsurgery site. We test our approach in a proof-of-concept study deploying MILiMAC inside a test platform to deliver and steer a $600 \boldsymbol{\mu m}$ ferromagnetic microbead. The bead is steered along a set of user-defined trajectories using closed-loop position control. Across all trajectories the best performance metrics are the mean error of $0.41 \mathrm{~mm}$ and the steady-state error of $0.27 \mathrm{~mm}$.
\end{abstract}

Adapted from:

J. Sikorski, S. Mohanty, S. Misra. MILiMAC: Flexible catheter with miniaturized electromagnets as a small-footprint system for microrobotic tasks, IEEE Robotics and Automation Letters vol. 5, no. 4, pp. 5260 - 5267, 2020 


\subsection{Introduction}

Microrobotic surgery is a future medical technology with the potential of revolutionising the way clinicians interact with human body. Employing functional miniaturized agents and controlling them in vivo could allow for localized and selective engagement of human body on cellular and tissue organization levels [105]. Such capabilities would complement contemporary medicine, which excels in procedures targeting entire organs, as well as in systemic treatment through delivery of therapeutic substances via the bloodstream.

The potential of microrobotic surgery has been so far predominantly demonstrated in an abundance of in vitro experiments performed with miniaturized milli- and micro-agents. These agents are often designed with a specific surgical tasks in mind and fabricated using sophisticated techniques [104]. Prominent examples involve: soft grippers for single-cell biopsy [227], scaffold-type robots for stem cell manipulation [228], thermoactive polymers for targeted drug delivery [229], stress-engineered MEMS microrobots [230], tubular micromotors, such as spermbots or microjets [231], and helical microswimmers [232]. With recent advancements in soft materials and microfabrication techniques, the surge of new micro-agents is expected to continue [233].

Due to their size, a vast majority of these micro-agents cannot rely on internal power sources and sensors for actuation and localization. Hence, they need auxiliary robotic infrastructure to assist them in successful operation [102]. In contrast to the rapid advancements in the design of micro-agents, as described above, the driving concepts behind the auxiliary infrastructure have not changed significantly in the last decade.

Clinically-relevant sensing of micro-agents remains an ongoing challenge, hampering attempts for controlling them in vivo [234]. Techniques used to actuate micro-agents, predominantly employing magnetic interaction, are somewhat more mature [103]. Nevertheless, in most cases they still follow the trend initiated by the OctoMag system, using sources of external magnetic field located outside of the body to exert wrenches on magnetic domains within the agents $[101,107,108]$. Modifications of this approach involve the use of mobile magnetic sources, however, these have been predominantly used for non-contact actuation of mesoscale medical devices [110,183,225].

Despite their prevalence in literature and success with various micro-agents during in vitro experiments, magnetic actuation systems located outside of the patient are inherently burdened with major disadvantages. Large electromagnets are required to generate adequate magnetic fields in deeply seated regions, such as the heart or the stomach [217]. This is particularly problematic when employing static coils, as the workspace of such a system must span a significant portion of human body [116]. Since the task workspace for micro-agents is several orders of magnitude smaller, these systems can be considered overscaled for that purpose. This situation is particularly challenging in presence of internal biological motion, which involves large displacements of tissues around the surgery site due to respiration, peristalsis or blood flow [32].

Furthermore, even the sheer delivery of micro-agents into a desired workspace remains challenging. Current literature usually assumes the micro-agents would be 
injected into the bloodstream and guided over large distances to the desired, deeply seated location [235]. These distances are several orders of magnitude larger than the micro-agents themselves. Free release of the agents into the body inherently subjects them to a variety of unpredictable biomechanical forces present in complex in vivo environments [234]. Due to related challenges, contemporary approaches to delivery and control of micro-agents have yet to provide plausible scenarios of clinically-relevant microsurgical procedures, making room for an alternative.

In this chapter, we reconsider the classical infrastructure used for delivery and control of micro-agents. We demonstrate that the forces available for microrobotic control increase as the magnetic actuation system, along with the available workspace are scaled down in size. The implications of that motivate stepping away from using electromagnets located outside the patient. Instead we propose to miniaturize them to the size of a few millimetres. The small footprint of such devices enables their delivery directly into the microsurgery site using tools for minimally-invasive surgery. Magnetic actuation systems integrated on catheters, endoscopes and needles have a potential of bridging the scales of the clinician and the agents and cells, providing well-defined and stable workspace for microsurgeries within the body.

We demonstrate the feasibility of this approach creating a system for steering of micro-agents, which is inspired by endovascular catheters and endoscopes [134]. Microrobotic Infrastructure Loaded into Magnetically-Actuated Catheter (MILiMAC) (Figure 7.1) is a device with three miniaturized electromagnets, which can be delivered to a deeply seated microsurgery site and deployed, creating a twodimensional target workspace. Within that workspace, MILiMAC can be used to actuate a wide range of micro-agents.

We test MILiMAC in an experimental scenario involving delivery and control of a ferromagnetic microbeads. Such microbeads are reliable micro-agents, which can be purposed to carry bioactive substances [236]. The catheter is inserted into a target workspace located at the distal end of a long channel imitating an anatomical duct. The bead is injected into that workspace through MILiMAC and controlled using miniaturized electromagnets along a set of predefined trajectories. Optical tracking is used for closed-loop control. Nevertheless, we also discuss the possibility of using on-site clinically-relevant modalities.

The rest of the chapter is structured as follows. Section 7.2 introduces the concept of miniaturized electromagnets, presenting its advantages over current approach. Section 7.3 presents the design of MILiMAC. Section 7.4 highlights the methods of the proof-of-concept study, including experimental setup. The control algorithm utilizing magnetic forces to move a microbead with MILiMAC is presented in Section 7.5. Section 7.6 contains the results of proof-of-concept validation and the discussion that follows. Finally, the entire chapter is summarised by Section 7.7, proposing next steps to bring our approach closer to clinical practice.

\subsection{The Effect of Scale on Magnetic Actuation Systems}

Magnetic interaction is exploited extensively as a principal method of non-contact actuation of micro-agents. It occurs between any micro-agent with magnetic prop- 


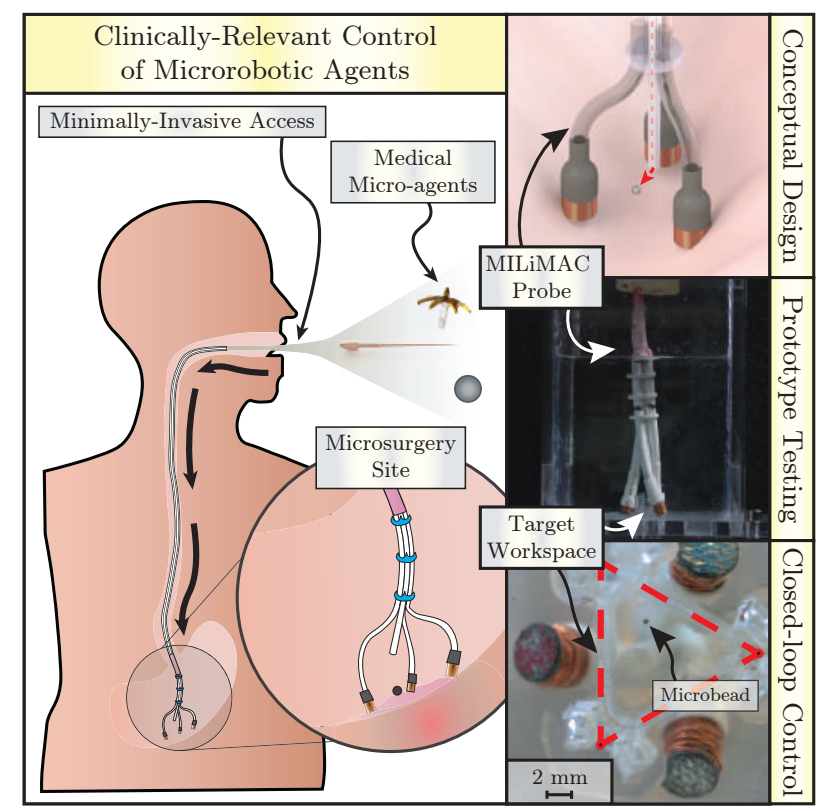

Figure 7.1: In this chapter, we exploit the concept of electromagnet miniaturization, creating Microrobotic Infrastructure Loaded into Magnetically-Actuated Catheter (MILiMAC). To our best knowledge, MILiMAC is the first device capable of providing localized means of steering magnetic micro-agents from within the human body. For that purpose, MILiMAC is inserted into the body in a minimally-invasive fashion and deployed at the site, where the microsurgery is to be conducted. Subsequently, various micro-agents can be delivered into the target workspace created by the catheter, and steered using miniaturized electromagnets.

erties represented by magnetic dipole moment $\left(\mathbf{m}_{a} \in \mathbf{R}^{3}\right)$ and the external magnetic field $\left(\mathbf{B}(\mathbf{p})=\left[\begin{array}{lll}B_{x} & B_{y} & B_{z}\end{array}\right]^{T} \in \mathbf{R}^{3}\right)$ at the location of the agent $\left(\mathbf{p} \in \mathbf{R}^{3}\right)$. Under the influence of that field, micro-agent experiences a magnetic wrench as described by (1.1). This wrench can be controlled by influencing $\mathbf{B}(\mathbf{p})$ and its spatial gradient using magnetic actuation systems comprising of electromagnets or permanent magnets.

The requirements imposed on magnetic actuation systems are agent-specific, due to diverse methods for using magnetic wrenches to achieve the desired behaviour of a particular agent. Nevertheless, in majority of applications one of the principal design criteria involves making the system capable of generating sufficiently high wrenches $(\mathbf{W})$, thus maximizing magnetic fields/gradients. Conventional way of solving this requirement involves maximizing the power of magnetic field sources. Exploiting the effects of scale on magnetic actuation systems offers an alternative solution.

While considering a simple point-dipole model, the resulting distribution of the magnetic fields remains constant, whereas the gradients decrease by the same factor by which the source is scaled [101] However, this conjecture does not precisely describe the behaviour of cored electromagnets. As they are complex devices, their magnetic field distribution depends on factors like the number of windings 
(determined by wire diameter), which do not necessarily scale in a straightforward fashion. Therefore, to represent their behaviour more accurately, we create a scalable FE model (Figure 7.2) of an iron-core electromagnet acting on a bead located at distance $\left(\mathbf{d} \in \mathbb{R}^{+} 3\right)$ using the approach presented in Chapter 3 . The distance (d) is scaled by the same factor the geometry of the coil, to show the effect of changing workspace. We estimate magnetic field $(\mathbf{B}(\mathbf{d}))$ under constant current for a range of $k$. Furthermore, for each simulation we also measure $\left(\nabla \mathbf{B} \in \mathbb{R}^{3 \times 3}\right)$ indirectly, by estimating the magnitude of the magnetic force $\left(\mathbf{F}_{\mu}\right)$ acting on a constant-size permanent magnet at $\mathbf{d}$.

The simulations performed with our scalable model indicate that, while the magnetic field distribution does depend on a particular scaling method (Figure 7.2) for wire diameter, the magnitude of the magnetic force $\left(\mathbf{F}_{\mu}\right)$ decreases sharply with $k$ in all simulated cases. As the scale of the model increases from 1 to 20, the forces are reduced in size by three orders of magnitude. This notion indicates that miniaturization of systems for magnetic actuation is a valid way of increasing the

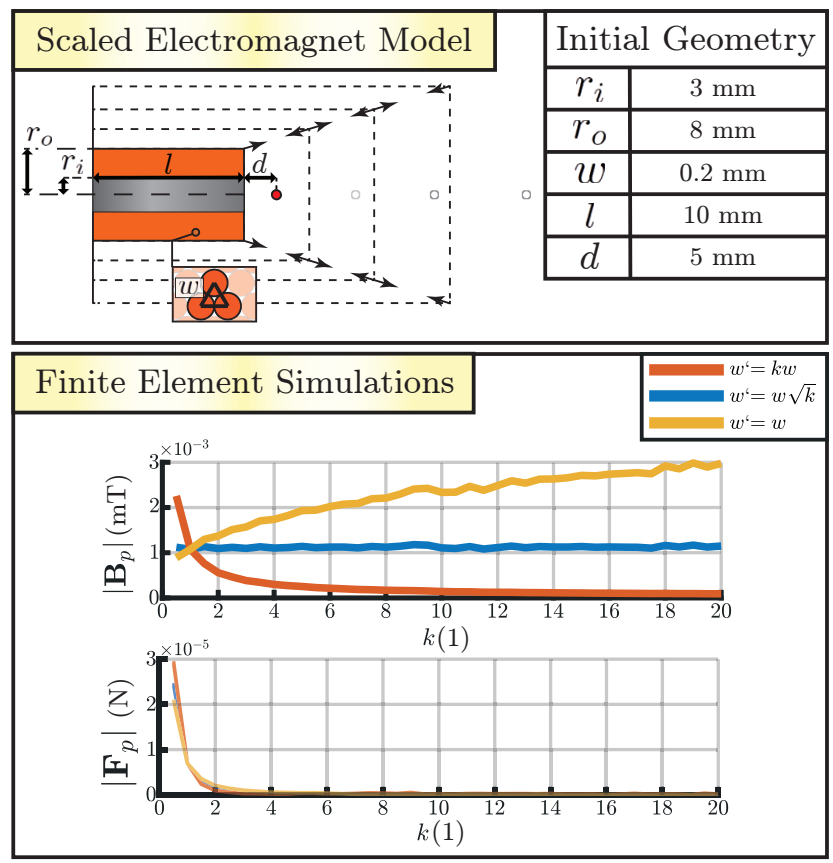

Figure 7.2: The results of finite-element (FE) simulations showing the effects of scale on the magnetic field generated by an iron-cored electromagnets. This simulation employs a scalable model following the convention proposed in Chapter 3 . In the model a scalable electromagnet is defined by the radius of the core $\left(r_{i} \in \mathbb{R}_{+}\right)$, outer radius of the coil $\left(r_{0} \in \mathbb{R}_{+}\right)$, wire diameter $\left(w \in \mathbb{R}_{+}\right)$and length $\left(l \in \mathbb{R}_{+}\right)$. We select and a measurement point (marked with red dot) at a distance $\left(d \in \mathbb{R}_{+}\right)$, at which we measure the magnitudes of magnetic field $\left(\left|\mathbf{B}_{p}\right| \in \mathbb{R}_{+}\right)$and magnetic force $\left(\left|\mathbf{F}_{p}\right| \in \mathbb{R}_{+}\right)$to be exerted on an iron sphere with a diameter of $1 \mathrm{~mm}$ located at the measurement point. We use the model to run a series of simulations, where the initial geometry of the model is scaled by a given constant factor $(k=0.5,1,1.5, \ldots, 20)$, with three different laws for the scaled wire diameter $\left(w^{\prime} \in \mathbb{R}^{+}\right)$. The distance $(d)$ is scaled by the same factor as the coil to preserve the relative location of the workspace. Our study show that the forces available for magnetic actuation can be significantly increased by miniaturizing the electromagnets. 

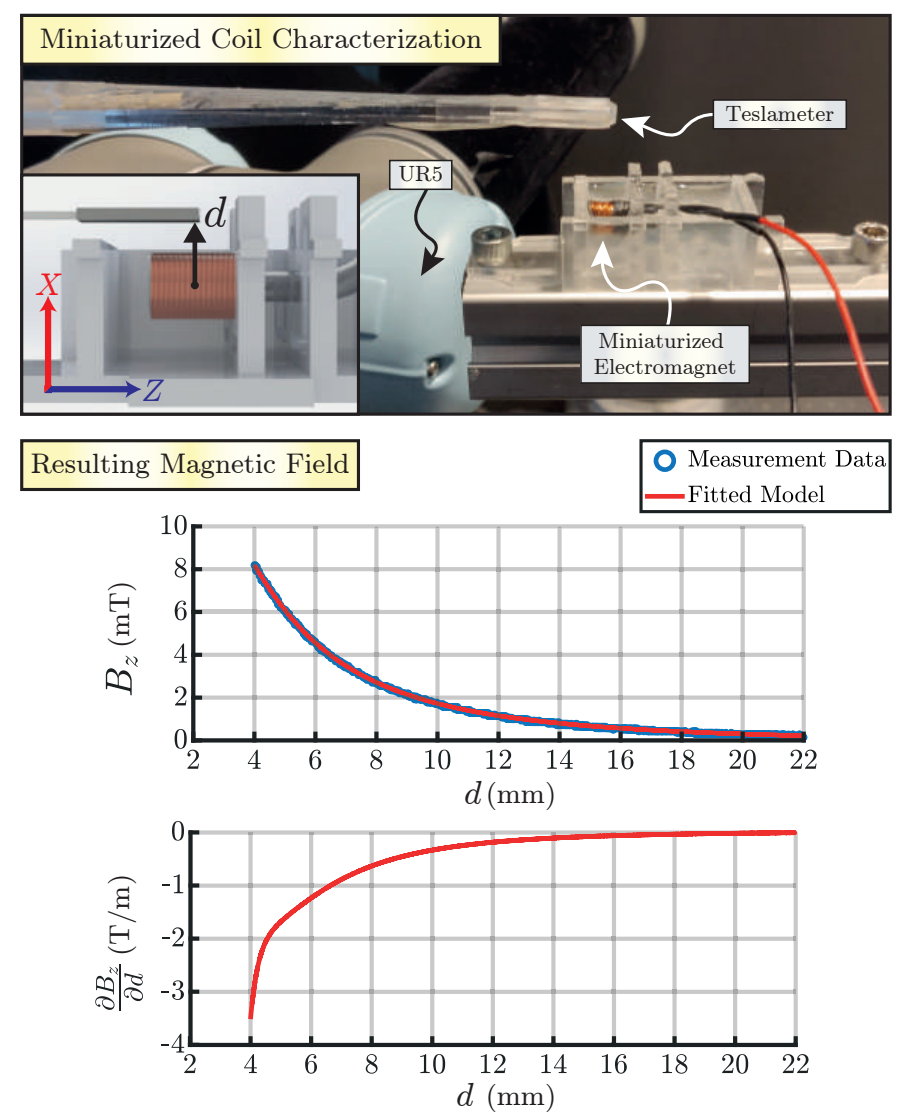

Figure 7.3: We characterize a prototype miniaturized electromagnet by measuring the magnetic field $\left(B_{z} \in \mathbb{R}\right)$ it generates along the distance $\left(d \in \mathbb{R}^{+}\right)$at $1 \mathrm{~A}$. The measurements are performed with a Senis 3MH3A-500MT (Senis AG, Baar, Switzerland) teslameter mounted on UR5 robotic arm (Universal Robots, Odense, Denmark). Furthermore, we obtain magnetic field gradient $\left(\frac{\partial B_{z}}{\partial d} \in \mathbb{R}\right)$ by fitting a rational function over the measured data.

magnetic gradients available for steering of micro-agents.

We test this experimentally, by characterizing a prototype miniaturized electromagnet (see Figure 7.3). Each dimension of the magnet is approximately one order of magnitude smaller than the ones used in conventional systems, which reduces the total volume occupied by it by three orders of magnitude. The results confirm the findings from $\mathrm{FE}$ analysis. Rated at a maximum of $2 \mathrm{~A}$, our miniaturized electromagnet provides gradients of up to $2-3 \mathrm{~T} / \mathrm{m}$ and fields of up to 10-12 $\mathrm{mT}$, which is comparable with the state-of-the-art systems discussed in [108]. In the same time, the electromagnet has largely minimized footprint and low power consumption of approx. $6 \mathrm{~W}$. Using such actuators as building blocks in magnetic actuation systems can reduce the means necessary for microrobotic control. 


\subsection{MILiMAC: Flexible Catheter with Miniatur- ized Electromagnets}

Despite the advantages discussed in previous section, magnetic actuation employing miniaturized electromagnets necessitates that the system is deployed at a distance of a few centimetres from the microsurgery site to be effective. This requirement inherently inspires revision of the classical paradigm, whereby the auxiliary magnetic actuation system is located outside of the body of the patient. Utilizing the small footprint of the miniaturized electromagnets, we propose to bring them close to the microsurgery site, a task possible using modern flexible surgical tools. As a consequence, we provide an alternative, minimalist approach to magnetic actuation.

We propose to use a flexible catheter to introduce miniaturized electromagnets into deeply seated body regions in a minimally invasive fashion. The minimum viable design of MILiMAC (Figure 7.4) employs three electromagnets on flexible tethers fitted at a distal end of a multilumen catheter, which also provides a central channel used for delivery of micro-agents. The sleek shape of MILiMAC allows for insertion into the microsurgery site in a minimally-invasive fashion through the natural orifices of human body (Figure 7.5) Upon reaching this site, the catheter is opened by running identical currents run through all coils. The resulting repulsive magnetic forces deflect MILiMAC tethers away from one another. Open catheter is positioned within the microsurgery site, using friction to lock the coils in place. This way, we form a well-defined workspace, into which micro-agents are subsequently delivered through the central channel of MILiMAC.

In contrast to classical, macroscale magnetic actuation systems, symmetry axes of all MILiMAC electromagnets are parallel. During the proof-of-concept validation presented in the next section, we use ferromagnetic microbeads, which can readily be manipulated with parallel electromagnets by exploiting magnetic forces (Figure 7.4). Despite their simple composition, microbeads can be coated with a wide range of substances to realise tasks such as targeted drug delivery [237].

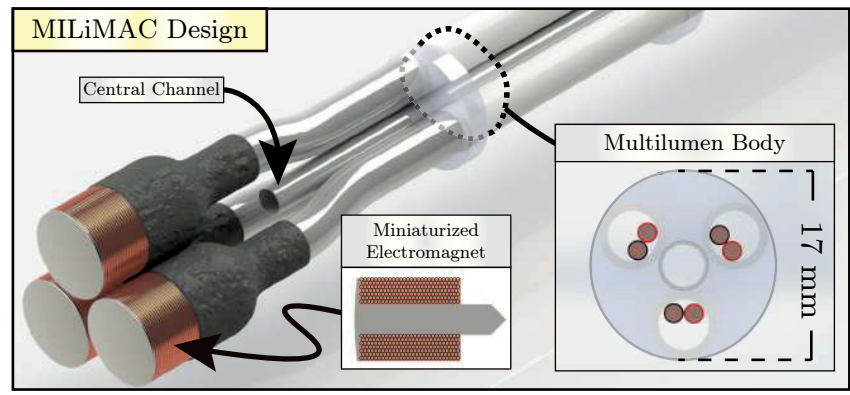

Figure 7.4: MILiMAC (Microrobotic Infrastructure Loaded into Magnetically-Actuated Catheter) is a proof-of-concept device, demonstrating the clinical relevance of localized magnetic actuation of micro-agents using miniaturized electromagnets. Three of such electromagnets are distributed radially along a central channel of MILiMAC, which is used for micro-agent delivery. Due to its flexible multilumen body, MILiMAC can be inserted into natural orfices of human body, delivering microrobotic infrastructure to deeply-seated regions. 


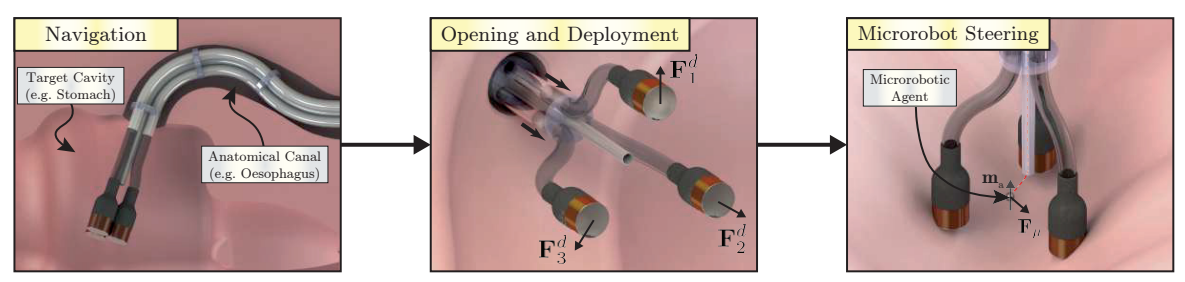

Figure 7.5: The proposed mode of operation of MILiMAC (Micorobotic Infrastructure Loaded into Magnetically-Actuated Catheter). The sleek shape and compliant structure of MILiMAC enable navigation to microsurgery sites through natural anatomical canals to deliver miniaturized electromagnets into deeply seated regions, such as stomach. Upon reaching the site, the catheter is deployed by running equal currents through all coils. The resulting repulsive forces $\left(\mathbf{F}_{i}^{d} \in \mathbf{R}^{3}\right)$ open MILiMAC by deflecting the electromagnet tethers away from one another. Open MILiMAC can be pressed against a surface, using friction to lock the coils in place and form target workspace. In this study, we use MILiMAC to steer ferromagnetic microbeads, as these micro-agents display both clinical relevance and properties favourable for magnetic steering. When placed in external magnetic field, a microbead becomes magnetized, with a resulting magnetic dipole moment $\left(\mathbf{m}_{a} \in \mathbf{R}^{3}\right)$. Such a magnetized microbead experiences magnetic force $\left(\mathbf{F}_{\mu} \in \mathbf{R}^{3}\right)$ dependent on gradients of the field at its location. We control this force by steering currents within MILiMAC coils.

\subsection{Proof-of-Concept Validation}

Since our catheter is designed to eventually become a clinically-relevant tool, we create an experimental setup to demonstrate the operation of MILiMAC in a procedure emulating the full workflow demonstrated in Figure 7.4. Albeit not as challenging, as in vivo deployment and control, this experiment highlights the advantages of MILiMAC, serving as a foundation for future development towards clinical use.

The prototype of MILiMAC and the experimental setup for proof-of-concept validation are shown in Figure 7.6. The catheter comprises of three miniaturized electromagnets with the dimensions as in Section 7.2. The coils are fitted on silicone tethers (length $100 \mathrm{~mm}$, diameter $4.8 \mathrm{~mm}$ ) located symmetrically around a HDPE (high-density polyethylene) 7.5 Fr. endovascular sheath (Maquet, Mahwah, NJ, USA) used as a central channel. The proximal end of the central channel provides a syringe port, used for injection of $600 \mu \mathrm{m} 440 \mathrm{C}$ stainless steel microbeads (MiSUMi Corporation, Tokyo, Japan) into the target workspace. Each miniaturized electromagnet is powered by a dedicated iPOS4808 BX-CAT servo drive (Technosoft S.A., Neuchâtel, Switzerland). The current used during the microrobot steering is limited to a maximum $2 \mathrm{~A}$, whereas during the deployment phase a current of $6 \mathrm{~A}$ is used to deflect the tethers of MILiMAC. The drives are connected to a research laptop through EtherCAT network (control rate $650 \mathrm{~Hz}$ ).

The target workspace has been delimited by a triangular reservoir located at the bottom of a larger acrylic tank filled with water. The catheter is inserted into the tank by an opening in its top wall, accessed through a $0.4 \mathrm{~m}$ long silicone tube imitating an anatomical duct, such as an oesophagus. The operator inserts MILiMAC through the tube, deploys the tethers as shown in Figure 7.4. and positions it around triangular reservoir. Once the catheter is in place, a ferromagnetic microbead is injected through the central channel.

FLIR Blackfly S-USB3 camera (FLIR Systems, Wilsonville, OR, USA) is used 
to image the workspace from below through the transparent floor of the container. The position of the agent is estimated by a segmentation-based tracker similar to our previous work [108]. The resulting information, along with real-time feedback on the location of the microbead are employed to steer it along a set of predefined trajectories. To achieve this, we develop a closed-loop position control algorithm presented in the following section.

\subsection{Position Control using MILiMAC}

The key part of the experiment involves autonomous steering of a microbead using coils provided by MILiMAC. This task is executed on a 2D plane, which we assume to be horizontal. This allows us to neglect the effect of gravity.

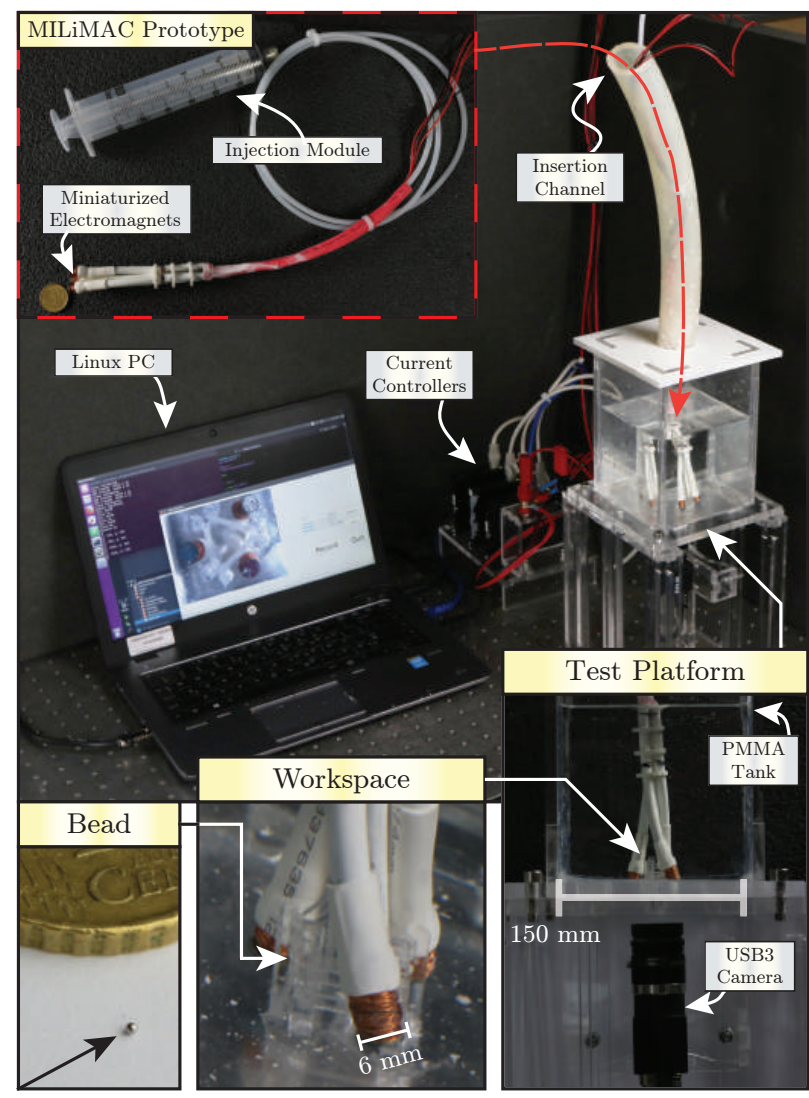

Figure 7.6: MILiMAC (Micorobotic Infrastructure Loaded into Magnetically-Actuated Catheter) is validated using a custom test platform, which comprises of a target workspace at the bottom of an acrylic tank. A prototype of MILiMAC is inserted into the tank through a long channel, imitating an anatomical duct. It is deployed around the workspace and monitored by FLIR BlackflyS USB3 Camera (FLIR Systems. Wilsonville. USA). Subsequently, the injection module comprising of a valved syringe at the proximal end of MILiMAC is used to deliver a $600 \mu \mathrm{m}$ microbead into the workspace. Finally, the bead is steered along a set of trajectories, using closed-loop control. 
Under such conditions, the steering task reduces to tracking a planar trajectory, defined as a set of points $\left(\mathbf{p}_{r} \in \mathbb{R}^{2}\right)$. We realise this task by implementing a closedloop position controller, which utilizes feedback from the optical tracking system. This feedback includes the location of the microbead $\left(\mathbf{p}_{a} \in \mathbb{R}^{2}\right)$, and the position $\left(\mathbf{p}_{i} \in \mathbb{R}^{2}\right)$ of each coil $(i=1,2,3)$ with respect to the global frame of reference. This frame is shown in Figure 7.7, which shows the task space as seen by the camera and illustrates the approach we propose.

The total magnetic field at $\mathbf{p}_{a}$ is the superposition of contributions from all coils. As a result, the bead becomes magnetised and gains magnetic dipole moment $\left(\mathbf{m}_{a}\right)$, with the value proportional to the field up to saturation, which usually happens in the range of 1-2 T. Nevertheless, to avoid quadratic dependence of the actuation force on the magnetic field we assume that $\mathbf{m}_{a}$ is constant and naturally aligned with the magnetic field, which is a common approach taken in the subject [227]

As gravity prevents off-plane motion of the microbead, it is useful to assume that all magnetic elements taking part in the interaction: the ferromagnetic microbead and the miniaturized electromagnets, are parallel and located on the task plane. The configuration of MILiMAC electromagnets (as shown in Figure 7.4) restricts the possible values of the magnetic field to

$$
\mathbf{B}(\mathbf{p})=\left[\begin{array}{lll}
0 & 0 & B_{z}
\end{array}\right]^{T} .
$$

We use this assumption to transform the equation (1.1). We derive the model relating planar magnetic force $\left(\mathbf{F}_{a} \in \mathbb{R}^{2}\right)$ exerted on the microbead by MILiMAC

$$
\mathbf{F}_{a}=\left\|\mathbf{m}_{a}\right\|\left[\frac{\partial B_{z}}{\partial x} \frac{\partial B_{z}}{\partial y}\right]^{T}=\left\|\mathbf{m}_{a}\right\|\left[\boldsymbol{\beta}\left(\mathbf{p}_{1}\right) \boldsymbol{\beta}\left(\mathbf{p}_{2}\right) \boldsymbol{\beta}\left(\mathbf{p}_{3}\right)\right] \mathbf{I},
$$

to the currents $\left(\mathbf{I} \in \mathbb{R}^{3}\right)$ through miniaturized electromagnets. The coil specific model $\left(\boldsymbol{\beta}\left(\mathbf{p}_{i}\right) \in \mathbb{R}^{2}\right)$ corresponds to the gradients per unit current calculated using the data from coil characterization (Figure 7.3).

We control the system by using the following modification of the standard PI law:

$$
\mathbf{F}_{a}(\mathbf{e})=\left(K_{p}\|\mathbf{e}\|+K_{i} \int_{t_{0}}^{t}\|\mathbf{e}\| d t\right) \widehat{\mathbf{e}}
$$

to calculate the virtual actuation force in the direction of the error $\left(\mathbf{e}=\mathbf{p}_{r}-\mathbf{p}_{a}\right)$. The magnitude of the actuation force is controlled by the PI action, with performance defined by gains $\left(K_{p}, K_{i} \in \mathbb{R}_{+}\right)$. The total value of the integral control is reset whenever $\|\mathbf{e}\|$ falls below $0.1 \mathrm{~mm}$. The model (7.2) can be inverted to map any $\mathbf{F}_{a}$ to corresponding control currents $(\mathbf{I})$.

Inverting the model (7.2) using Moore-Penrose pseudoinverse is likely to provide a solution assigning negative current to at least one of the coils. As observed experimentally, this situation leads to unpredictable violations of assumption about constant value and direction of $\mathbf{m}_{a}$, causing stability problems and requiring alternate control framework, beyond the proof-of-concept scope of this chapter. [108] Nevertheless, the operation of MILiMAC with simple 


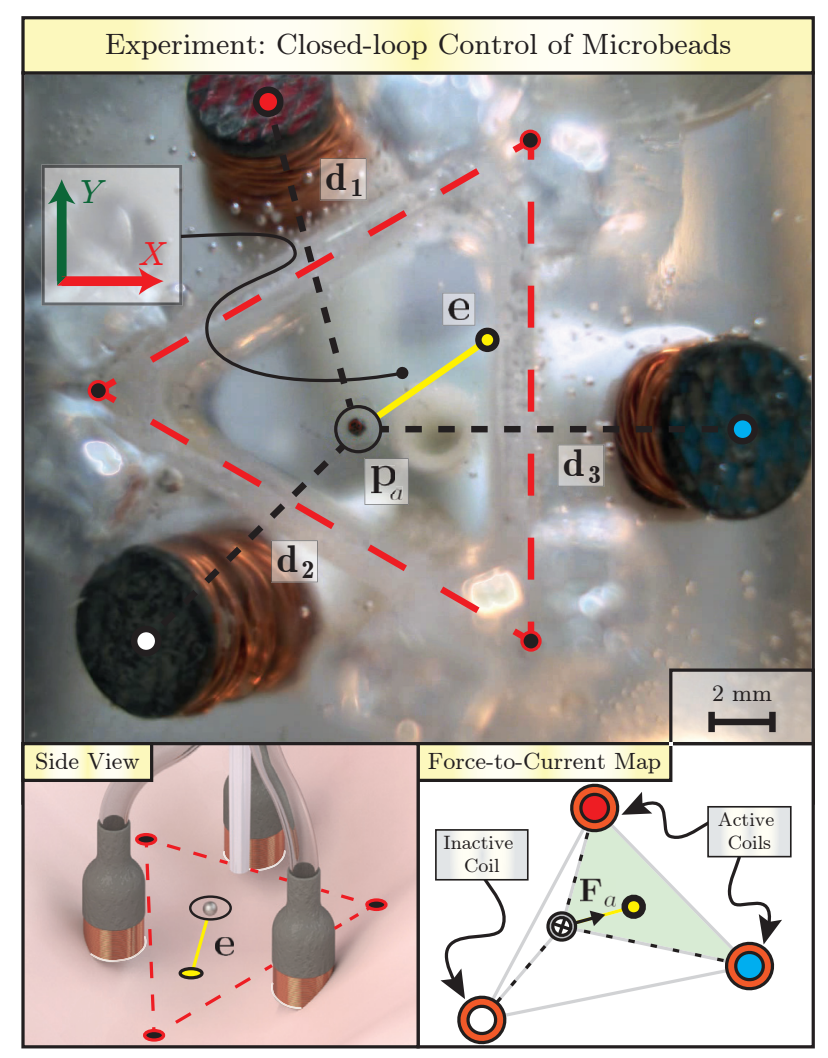

Figure 7.7: MILiMAC (Micorobotic Infrastructure Loaded into Magnetically-Actuated Catheter) is used to steer ferromagnetic microbead along point-to-point trajectories (yellow dot) employing closed-loop control. The catheter deployed around a triangular reservoir (red lines). Tracking system is used to provide locations (red, white and blue dots) of MILiMAC coils $(i=1,2,3)$ and the position $\left(\mathbf{p}_{a} \in \mathbb{R}^{2}\right)$ of the microbead. This information is used to calculate: the error $\left(\mathbf{e} \in \mathbb{R}^{2}\right)$ between actual and reference position, and the relative distances $\left(\mathbf{d}_{i} \in \mathbb{R}^{2}\right)$ from the microbead to each coil. These distances are required by a force to current map relating the output of a PI controller to the currents in MILiMAC coils. The map employs selective activation of coils to rely on pulling for force generation.

PI controller can still be effectively realised by introducing an intermediate step, in which only two coils of MILiMAC are selected to actuate the bead at any instance of time. These coils are selected based on the direction of the force $\mathbf{F}_{a}$, such that the magnetic interaction is used solely for pulling (see Figure 7.7). We use that property in our control scheme to map the actuation force to only the currents $\left(\mathbf{I}_{a} \in \mathbb{R}_{+}^{2}\right)$ for the selected coils $\left(a_{1}, a_{2} \in i\right)$ as follows:

$$
\mathbf{I}_{a}=\frac{1}{m_{a}}\left[\boldsymbol{\beta}\left(\mathbf{d}_{a_{1}}\right) \boldsymbol{\beta}\left(\mathbf{d}_{a_{2}}\right)\right]^{-1} \mathbf{F}_{a},
$$

whereas keeping third coil unpowered. The resulting currents are always positive, ensuring predictable magnetization of the microbead.

The forces contributing to static friction are a significant factor limiting reliable motion of ferromagnetic beads smaller than $1.2 \mathrm{~mm}$ in large parts of the workspace. 
We provide a solution to that problem by revisiting the assumption made earlier in this section on absence of magnetic torque within our actuation technique. Instead of driving a coil $(i)$ with steady current $\left(I_{i}\right)$ corresponding to the solution of (7.4), we use the following oscillatory currents instead

$$
I_{i}^{o}(t)=I_{i} \sin \left(2 \pi \omega_{i} t+\phi_{i}\right) .
$$

Both the frequency $\left(\omega_{i} \in \mathbb{R}_{+}\right)$and the phase $\left(\phi_{i} \in \mathbb{S}\right)$ of the currents are synchronized for each coil.

Under such conditions, the values of $I_{i}^{o}(t)$ oscillate continuously in a synchronous manner, crossing the zero point simultaneously. When the crossing happens, the microbead experiences short-lasting magnetic torque. This effect is used to perturb the microbead, generating rolling motion, which overcomes the static friction. As the currents increase, the bead re-magnetises and follows in direction of the magnetic force $\left(\mathbf{F}_{a}^{o}(t)=\mathbf{F}_{a} \sin \left(2 \pi \omega_{i} t+\phi_{i}\right)\right)$ acting on the bead [238].

\subsection{Results and Discussion}

The results of the proof-of-concept study are presented in Figure 7.8. The control algorithm presented in the previous section is implemented in the experimental setup presented in Figure 7.6. The control cycle is timed at $45 \mathrm{~Hz}$, limited by the frame rate of the optical camera.

Our control algorithm is used to drive the currents inside the miniaturized electromagnets of MILiMAC. The resulting magnetic force acting on the bead affects the direction and the magnitude of its rolling motion. As a result, the microbead successfully accomplishes three trajectories defined by the user. These trajectories comprise of a number of target points, arbitrarily selected through the user-guided interface.

Across three representative trajectories presented in this chapter the largest mean error is $0.81 \mathrm{~mm}$ for Trajectory 1 . Given these values are comparable to the size of the microbead itself $(0.6 \mathrm{~mm})$, and much smaller than the total size of the workspace (triangle side $16 \mathrm{~mm}$ ), we can consider our steering successful. The steady-state behaviour of our system is generally better than the transient one due to lack of high-frequency oscillations, visibly introduced by the rolling motion. Thus, we additionally quantify the error only during these intervals, when the reference location position remains constant. During these intervals, the largest mean error across all trajectories is reported to be $0.41 \mathrm{~mm}$.

When using electromagnets inside human body, care has to be taken to ensure that the thermal energy dissipated by these devices does not damage the surrounding tissues. We quantify that energy by calculating the root mean square power (RMS) dissipated for each coil during the time intervals, when it is active. We do that using the data describing the instantaneous coil currents along each trajectory, as prescribed by (7.5). The maximum RMS dissipated power is 0.96 $\mathrm{W}$, which is almost two orders of magnitude less than the power used in destructive procedures, such as cardiac ablation (50 W) [75] This allows us for positive evaluation of the thermal safety of MILiMAC.

The results of the proof-of-concept study indicate, that miniaturized electromagnets constitute a promising alternative to macroscale magnetic actuation sys- 


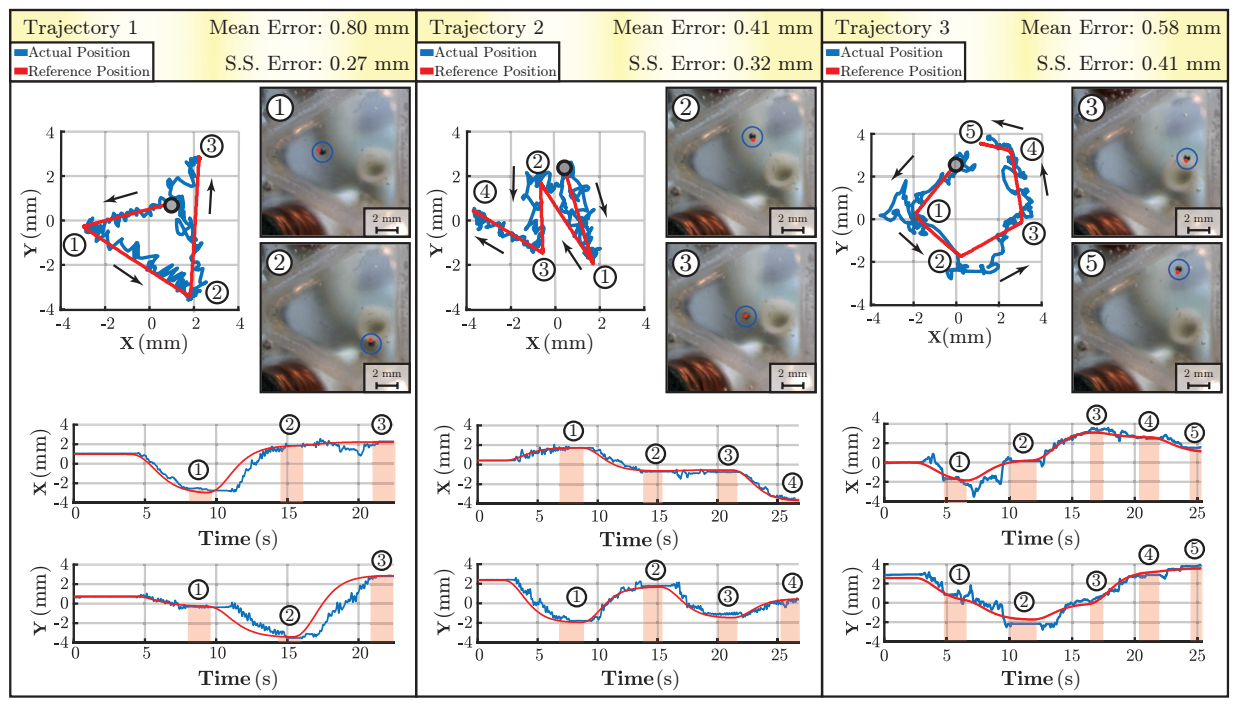

Figure 7.8: We test the prototype of MILiMAC by controlling the microbead along a set of point-to-point trajectories. Three representative trajectories are defined on-the-go by the user, who selects instantaneous target point using user-guided interface. Smooth trajectories between the target points (marked with numbers) are generated using low-pass filter. The top plots show the $2 \mathrm{D}$ position plot for each trajectory, whereas the bottom show the time evolution of both coordinates of the position of the microbead. Gray circles on each 2D plot are used to indicate the size of the microbead. For each trajectory the root mean square (RMS) heat dissipation of each coil (C1, C2, C3) and the mean position error is calculated. Since the steady-state (S.S.) error of the system is generally lower than the mean due to the rolling motion, we additionally calculate the error for the S.S. regions, marked with red trapezoids on the time plots.

tem. Located on instruments for minimally-invasive surgery, miniaturized electromagnets are capable of providing effective local magnetic actuation in deeply seated target areas. Furthermore, the study allowed us to identify several key limitations of our current concept, which have to be tackled as the next steps on the road towards clinical applications.

During the deployment phase, we have successfully managed to use the magnetic repulsion between the coils to deploy MILiMAC around the target workspace. Nevertheless, the approximate average deflection of a tether from the initial position, as measured from from the camera images, was only $14 \mathrm{~mm}$ for the current of 2 A. Therefore, imprecise manual manipulation at the base of the insertion channel was still required to position MILiMAC around the reservoir. A possible way of addressing that problem involves the use of externally-generated strong magnetic field around the target workspace to exert magnetic torques on the tethers. These torques would allow for large and precisely controlled deflections enabling positioning of MILiMAC tethers using techniques developed for steering magnetic catheters [116, 217]

Considering the results of the proof-of-concept steering experiment, the transient behaviour of the microbead still needs to be significantly improved, to reduce the steering errors to values comparable with macroscale magnetic actuation systems (0.1-0.2 mm). As our control approach involved a simple PI action with heuristically determined gains and a significantly simplified model of the magneti- 
zation of the bead, improvements could involve a more advanced controller, based on a realistic, quadratic model of the actuation [108]. The main factor degrading the performance of the system is significant static friction between the microbeads and the surface, which necessitates using imprecise rolling motion. As friction is inherently determined by the nature of contact between the agent and the environment, we expect the significance thereof to be reduced by using agents exhibiting low friction in body environment. Ultimately, the problem vanishes for procedures involving agents completely suspended in fluid environment.

Another important limitation involves phenomenon most readily visible in Trajectories 1 and 3, where the bead deviates extensively from the path at times. We attribute these deviations to the unmodelled influence from the unpowered coil, magnetized nevertheless due to cross-talk. Cross-talk is usually not observed in macroscale magnetic actuation systems employing standard configurations, such as these described in [107]. However, its significance increases at smaller scales, thus is likely to occur in our miniaturized electromagnets, especially if they are kept in parallel. This particular behaviour can be reduced by including hall-effect sensors and controlling miniaturized electromagnets in a closed-loop manner [217].

A separate challenge involves a possibility of electromagnet displacement within the target site, either due to biological motion, or mutual attraction of the magnets (conversely to repulsion used to deploy the system). In case of our proof-of-concept experiment, we rely on friction as well as on the presence of triangular reservoir within the workspace. In clinical setting this problem can be solved by either real-time tracking of electromagnet positions, or by integrating them on surgical instruments, such as needles, which offer improved stability with respect to the environment. For superficial procedures, miniaturized electromagnets could be potentially attached to the skin of the patient.

It is important at this point to stress that MILiMAC is a prototype device. We envision several ways, in which the concept of a surgical instrument with miniaturized electromagnets can evolve (Figure 7.9). Since our strategy assumes creation of a well-defined workspace, sheets of elastic polymeric film can be fitted between the tethers of MILiMAC to seal the workspace from physiological disturbance. Furthermore, millimetre-sized ultrasound transducers or optical devices could be integrated into the body of MILiMAC, or inserted through its central channel for local tracking of micro-agents and their environment [239,240]. Finally, additional off-plane electromagnets can be integrated into the body of MILiMAC to enable three-dimensional actuation of microrobots. Such actuation is desirable, as it not only ultimately allows for $3 \mathrm{D}$ control of microrobots, but can also be exploited for planar manipulation in situations, where the effects of gravity on the motion of the microrobot cannot be neglected.

\subsection{Conclusions}

In this chapter we reconsider the classical infrastructure used for control of medical micro-agents. Informed by results indicating that miniaturized electromagnets offer larger actuation forces than conventional macroscale system, we present MILiMAC. MILiMAC is equipped with three miniaturized electromagnets located on distal end of a multilumen catheter, and can be navigated into a deeply seated 


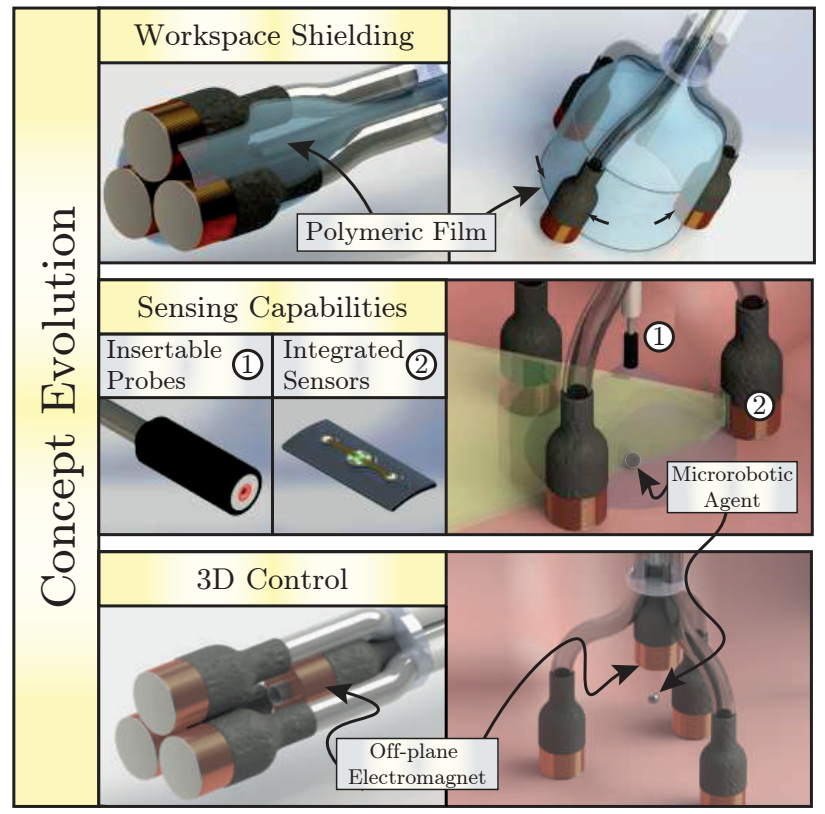

Figure 7.9: We envision the concept of a flexible catheter with miniaturized electromagnet to evolve gradually towards clinically-relevant designs. We propose three such improvements, which illustrate the potential of current design. First, the structure of the catheter can be used to support sheets of polymeric film to shield the microrobotic workspace from external disturbance, such as fluid flow. Secondly, catheter can be used as a platform for on-site sensing of the agents, using miniaturized optical and ultrasound devices. Finally, three-dimensional actuation of a micro-agent can be enabled, by integrating additional off-plane electromagnets into the body of the device.

microsurgery site in a minimally-invasive fashion. Upon deployment, MILiMAC creates a target workspace, in which magnetic micro-agents can be controlled using magnetic field generated by miniaturized electromagnets. We test MILiMAC in a proof-of-concept experiment, demonstrating closed-loop position control of a ferrmagnetic microbead using feedback from optical camera. 



\section{Part IV}

\section{Concluding Remarks}





\section{Chapter 8}

\section{Discussion}

In this dissertation we addressed important challenges related to the design, modelling and control of magnetic catheters, using robotic technology with the intention of bringing these devices closer to clinical practice. We explored a broad range of topics across a series of studies, generating a solid foundation, which can be used both as an inspiration in future academic pursuits, as well as applied commercially. This chapter serves to summarise our work, providing logical link between knowledge accumulated in individual studies and drawing general conclusions and discussing them with respect to the research objectives formulated in the introduction. Finally, to put our research in perspective, we express our opinion on future directions in which the field of magnetic catheters (and, more broadly, magnetically-actuated surgical instruments) is likely to evolve within the next decades.

\subsection{Conclusions}

The use of magnetic actuation opens up unique possibilities for development of robotic catheters with advanced functionalities. Magnetic interaction allows for direct and localized application of controlled forces and torques to the elements within the structure of the catheter in a non-contact fashion. The universality and versatility of the underlying principles makes them applicable in a wide range of clinical scenarios. Magnetic actuation can be effectively used to move the catheter, change its internal configuration, or make it interact with the environment directly or through deployable intermediate agents. Furthermore, a single set of structural elements is capable of realising several of these tasks, with the instantaneous functionality achieved through a particular interaction of the catheter and the auxiliary infrastructure used to generate external magnetic fields.

This broad landscape offers unprecedented freedom to designers of future medical instruments. By addressing the research questions formulated in Section 1.4, we have intended to strengthen the foundation, upon which advanced magnetic catheters can be developed, commercialised and put into effective clinical use. The key findings of the work presented in each part of this dissertation are: 


\subsubsection{Part I}

Magnetic catheters rely on external auxiliary infrastructure for their successful operation. Means of generating sufficient external magnetic fields must be made available in a workspace, which is large enough for a particular task. To approach RQ.1, we direct our efforts towards the development of clinically-relevant systems utilizing mobile electromagnetic coils. These coils, several orders of magnitude larger than the catheter itself in terms of volume, are located outside of the body of the patient. They are used to generate external fields which penetrate through the tissues of the body, transmitting power to the functional elements within the body of the catheter.

- In Chapter 2 we initiate our work developing BigMag, which is a prototype containing six mobile electromagnets revolving around a spherical workspace with a diameter of $100 \mathrm{~mm}$. A significant amount of work within this chapter has been devoted to formulating a kinematic model of a mobile coil array. Furthermore, we study the behaviour of BigMag using numerical and experimental techniques, ultimately creating an optimization-based inverse map, which allows us to generate prescribed magnetic fields. As a result, we obtain a reliable workbench system, capable of on-demand generation of three-dimensional magnetic fields with magnitudes of at least $40 \mathrm{mT}$ with the average error of $2.20 \%$. Additionally, we establish significant engineering expertise in the subject which facilitated more clinically-oriented further developments.

- In Chapter 3 the mobile coil approach has been used to realise an actuator for the ARMM system. Here, a single macroscale electromagnet has been used as an end-effector in a $6 \mathrm{DoF}$ robotic arm. The geometry of the electromagnet is calculated using an optimization routine to achieve maximum possible field for a limited payload. The resulting system is clinically-relevant. Even though the ARMM system is intended for the generation of magnetic fields at a maximal distance of $300 \mathrm{~mm}$ from the face of the coil, the robotic arm allows for repositioning of the coil at a distance of $1300 \mathrm{~mm}$ from its base. Thus, the ARMM system can be used to provide magnetic actuation in an effective workspace spanning the entire human body. In contrast to BigMag, the ARMM system can be used to generate both prescribed magnetic fields as well as gradients. We enable that by an iterative map, which accounts for saturation of the mobile electromagnet. By simultaneously controlling the position of the coil and the current running through it, we demonstrate maximum achievable gradients of $0.6 \mathrm{~T} / \mathrm{m}$ for a maximum field of $20 \mathrm{mT}$. Across a set of field/gradient trajectories, we achieve an average field error of $2.34 \%$ and an average gradient error of $7.20 \%$.

The main challenge related to creation of clinically-relevant auxiliary infrastructure is imposed by the unfavourable scaling properties of magnetic actuation. In principle, scaling the effective workspace available for operation of magnetic catheters by one order of magnitude requires a system with power consumption three orders of magnitude larger. By using the mobile coil approach, we are ultimately able to work around this limitation. 
Since, at any instance, the volume in which the catheter moves is relatively small (the tip of the catheter is rarely more than 10 centimetres long), auxiliary infrastructure does not necessarily need to generate fields spanning the entire human body at once. Using the mobile coils, we provide actuation in a much smaller workspace which nevertheless remains positioned around the catheter tip as it moves throughout the body. This way, effective operation of magnetic catheters can be realised using infrastructure with modest power requirements and much reduced financial costs. We reach this conclusion in two steps, each one producing in a fully operational magnetic actuation system as an outcome.

\subsubsection{Part II}

Successful development of reliable auxiliary infrastructure has enabled us to delve into mesoscale, performing a series of studies focused on the catheters themselves. Here, our efforts are eventually concentrated within two interdependent domains. On one hand, within the boundaries of research question RQ.2, we explore the design possibilities offered by magnetic actuations to create novel instruments with advanced functionalities. In parallel, a second body of work involved using the principles of (continuum) robotics, developing tracking (research question RQ.3) and control (research question RQ.4) solutions which make our catheters operational. This dual nature permeates the chapters presented in Part II. Each catheter design presented therein is in essence co-defined by the context of enabling robotic solutions, which are in part universal and transferable.

- Our first catheter is presented in Chapter 4. Despite having a structure which is much simpler than classical tendon-driven devices, it can be reliably steered in 3D using magnetic actuation provided by the BigMag system. The catheter is tracked using shape-from-silhouette technique, generating a pointcloud from stereo vision cameras. The continuous shape of the catheter is reconstructed, which an accuracy of $0.59 \mathrm{~mm}$. The mechanics of the catheter is represented using PRB modelling, which allows us to formulate an inversemodel controller based on quasi-static assumption. The controller is used to steer the catheter across a set of trajectories with the minimum reported mean position error of $0.57 \mathrm{~mm}$ (RMS error $0.77 \mathrm{~mm}$ ), and the maximum catheter tip speed achieved of $5 \mathrm{~mm} / \mathrm{s}$. To the best of our knowledge, this is the first demonstration of a magnetic catheter being controlled using the full-shape feedback and PRB modelling approach.

- In Chapter 5 we create a tentacle catheter for underactuated grasping. The tentacle catheter is selected out of three possible grasping strategies, based on its adherence to a set of design requirements. The catheter contains a permanent magnet at its tip and a proximally located miniaturized electromagnet. The section of the catheter between these two magnetic elements can be wrapped around a target object using external fields. By controlling the current within the miniaturized electromagnet, a stable and detachable underactuated grasp can be achieved. We test the prototype of our device inside BigMag, showing that it can apply up to $0.1 \mathrm{~N}$ of force to a target object. Finally, we demonstrate the validity of our approach by using the 
tentacle catheter for manipulation of porcine tissue. Our device is the first of its class, as no continuum manipulator has been applied in the literature for underactuated grasping in mesoscale before our study.

- Chapter 6 proposes means for ultrasound-guided targetted-drug delivery. Here, we develop a magnetic catheter with a millirobotic tip, containing a miniaturized electromagnet and a biodegradable capsule. The capsule is made out of a composite of hard magnetic material and Sudan Orange dye (imitating the active substance) suspended in gelatin. In the first part of the procedure, the capsule remains attached to the electromagnet, attracted by magnetic forces. The catheter is guided to the target site using external actuation provided by the ARMM system. Upon reaching the deployment position, the capsule is ejected, by reversing the direction of the current within the miniaturized electromagnet. We test our design in a proof-ofconcept experiment. Tracking of the catheter is realised using custom built scanning ultrasound, providing full 3D pose of the catheter with the accuracy of $0.37 \mathrm{~mm}$. We test the guidance of the catheter in a series of trajectories, with an lowest mean position control error of $0.82 \mathrm{~mm}$. Ultimately, we propose a null-space based controller for capsule targeting, reaching a designated target with a mean residual catheter displacement of $0.88 \mathrm{~mm}$.

From the design perspective (research question RQ.2), the most important paradigm shift occurring throughout the studies within Part II is our gradual transition from permanent magnets to miniaturized electromagnets as principal magnetic elements incorporated into the structure of the catheter. In contrast to simple permanent magnets, the properties of miniaturized electromagnets depend on currents running through them, which can be controlled directly. In a breakthrough step, we realise that incorporation of ferromagnetic cores into miniaturized electromagnets significantly amplifies the magnetic forces and torques they generate/experience, making them reliable and versatile actuators. This supposedly straightforward notion has so far been completely neglected in existing literature on magnetic catheters, which considered only coreless miniaturzed electromagnets for MRI-compatible actuation. [119,121,241,242]. The possibilities offered by electromagnets with ferromagnetic cores allow for designing of a wide range of reconfigurable magnetic catheters, thus opening up ways of achieving tasks unattainable otherwise. We are certain that our work only scratches the surface of what these elements can enable.

The robotics work within this part is essential to integrate the mesoscale catheter and the macroscale auxiliary infrastructure in each study into a single, autonomous robotic system. Two key contributions presented in this dissertation allow us to achieve this. First of all, we address research question RQ.3 by demonstrating that vision can be used to create a shape sensing framework for real-time 3D estimation of state of magnetic catheter. Secondly, we explore a PRB modelling approach to approximate the behaviour of our catheters. This approach allows us to develop controllers for realisation of given clinically-relevant tasks using magnetic actuation (question RQ.4). We put particular emphasis on the modularity of our solutions, which facilitates their transfer between studies. Our vision-based framework is used first with optical cameras and is then expanded to 
work with scanning ultrasound. The PRB model-based controller is first applied to relatively unconstrained actuation provided by BigMag and is later integrated within the more demanding context of the ARMM system.

\subsubsection{Part III}

The concept of miniaturized electromagnets utilised within this dissertation vastly expands the possible functional behaviour of catheters. As demonstrated in Chapters 5 and 6, such electromagnets can be used to achieve controlled displacement of other magnetic elements within the same catheter. In fact, this functionality bears resemblance to the way, in which we use the macroscale electromagnets of BigMag and the ARMM system, although at much smaller scale. Exploiting this analogy allows us to approach the topic of magnetic catheters from a different perspective. The result of that investigation - MILiMAC - relates both to research questions RQ.1 and RQ.2. It is a novel catheter, which can be externally actuated by macroscale auxiliary infrastructure, and which itself can be considered as mesoscale auxiliary infrastructure. Its main functionality is to bridge scales, providing localised magnetic actuation of yet smaller objects: the medical microrobotic agents.

- The proof-of-concept study introducing MILiMAC is presented in Chapter 7. Here, we first discuss the benefits of electromagnet miniaturization, showing that they are capable of generating sufficient magnetic fields and gradients for actuation of untethered microrobotic agents. We use three such electromagnets fitted at distal ends of flexible tethers to create the end-effector for the prototype of MILiMAC. We test our catheter in an experimental scenario., inserting it into a test-bed through a long channel imitating an anatomical duct. Subsequently, we deploy MILiMAC around a triangular reservoir, into which a $0.6 \mathrm{~mm}$ ferromagnetic microbead is introduced. We use MILiMAC to control the position of the microbead across user-selected trajectories, reporting the minimum mean error of 0.41 $\mathrm{mm}$ and the minimum steady-state error of $0.27 \mathrm{~mm}$.

Our work demonstrates that catheters can successfully be used to provide localised magnetic actuation in deeply-seated locations within human body. By using miniaturised electromagnets, large fields and gradients can be generated with power consumption much lower than in conventional macroscale auxiliary infrastructure, such as BigMag or the ARMM system. Devices such as MILiMAC open up a new potential route for clinical applications of microrobotic agents in the future.

\subsection{Outlook}

The work contained within this dissertation demonstrates that the principles of magnetic actuation can be successfully applied to create a wide range of robotic catheters. The subject of magnetic catheters is likely to evolve, with future studies bringing novel insights on how these devices can be used in clinical practice. 
Based on our experience, in this section we suggest the potential directions of that evolution.

\subsubsection{Magnetic Actuation Systems}

Our work presented in Part I addresses the challenge of generating sufficiently strong ( $>30 \mathrm{mT}$ ) magnetic fields in workspaces appropriate for clinical tasks. We believe that by using mobile electromagnetic coils, this problem can be alleviated within the next decades. Designs similar to our ARMM system, presented in Chapter 3 and applied in Chapter 6 , can in the future become an indispensable components of a surgical suite. Nevertheless, several aspects of magnetic actuation systems employing mobile coils still have to be improved, in order to unleash their full clinical potential and enable safe use in operating theatre.

- The capabilities of a clinical magnetic actuation systems can be significantly expanded by using more than one mobile electromagnet. The six coils of BigMag can perform that task relatively fast regardless of their instantaneous configuration, whereas the ARMM system requires mechanical reconfiguration, involving motion of the device. Furthermore, since certain positions of the electromagnet cannot be achieved (e.g. due to obstacles, such as the body of the patient or the operating table itself), the effective range of directions for the resulting magnetic field is limited.

Future studies can remedy these problems, by combining the advantages of the two systems. By using a larger number of independently-moving electromagnets, a more versatile magnetic field control can be achieved. Remarkably, three such mobile electromagnets used simultaneously in a fashion demonstrated in Chapter 3 can already provide independent, threedimensional control of fields/gradients acting on the catheter with considerably less motion required. The new DeltaMag system presented in the literature can be considered a step in this direction [225].

- Before transitioning the solutions based on mobile electromagnets into clinical context, extensive efforts have to be made to ensure their safety. Since the robotic arms positioning the electromagnets will have to work in the cluttered and dynamic environment of the operating theatre, routines have to be created to enable collaborative operation in the proximity of human actors. On-line mapping of the motion of clinicians and the patient within operating theatre can be integrated within the context of the magnetic actuation systems [243]. The resulting information can be used to create more advanced controllers of magnetic catheters, taking environmental constraints into account while positioning the mobile electromagnets [244].

- More complex motion strategies for the mobile electromagnets can be developed, helping effective field generation depending on a particular clinical scenario. In contrast to our approach, where the symmetry axis of the electromagnet is always aligned with the magnetic element on the catheter, techniques utilising more advanced field maps can be developed enabling 
the coils to be effectively used regardless of their poses. This way, independent simultaneous actuation of several magnetic elements (or even several catheters) could be achieved.

- Engineering work can be done on the mobile electromagnets themselves to increase the field generation. This involves both the design of the electromagnets themselves and the development of modules assisting their action. In particular, work on lightweight, efficient heat dissipation systems mountable on robotic arms can enable the use of electromagnets with smaller wire diameters and larger maximum currents [108]. Thereby, the magnetic actuation systems of the future could provide much stronger magnetic fields for extended periods of time without the risk of overheating.

The clinical magnetic actuation systems which successfully approach these challenges will become indispensable tools in the surgical practice of the future. The versatility of magnetic interaction can allow us to expect, that the possible application of these systems will not be restricted solely to catheters, as various other types of surgical instruments can be actuated in this fashion [102]. Ultimately, a cost-effective suite for magnetic actuation should involve a broad range of procedures in various medical disciplines. This way, the full potential of this mode of actuation can be exploited, helping the clinicians to treat patients faster and more effectively.

\subsubsection{Catheter Design, Tracking and Control}

The complex mechanical behaviour of various magnetic catheters developed in Part II of this dissertation leads us to expect that these devices can in the future vastly expand the possible set of tasks executed by the clinicians in challenging locations within the body. However, a relevant set of challenges within the state-of-the-art methodology still has to be tackled before the principles of magnetic catheters can be translated effectively to industry use. The improvements are possible both with respect to the techniques used in structural design of the catheters, as well as to the robotic technology used to track and control their state:

- The hard magnetic materials currently used within the structure of catheters negatively affect the safety of resulting devices. Recent breakthroughs in soft magnetic materials can in the future alleviate this problem [149]. By employing shape-programmable soft composites, the range of motion of the resulting magnetic catheters can be expanded. In the same time, the resulting devices will retain the favourable compliance matching with soft tissue. Moreover, the use of soft conductive materials can enable the creation of deformable miniaturized electromagnets, allowing the soft magnetic catheters of the future to perform as active devices, in line with the actuation techniques presented within this dissertation.

- The use of soft composites can also allow for future magnetic catheters to involve a higher degree of biomimetics. In particular, an analogy can be drawn between the catheters and various groups of internal human parasites. These organisms are particularly skilled in traversing large distances 
in various parts of human body, causing minimum tissue damage. In the same time, their elongated shape closely resembles the one of catheters. The strategies used by parasites to traverse the body could be realised using programmable magnetic matter, thus inspiring new generations of soft magnetic catheters.

- Irrespective of a design of a particular magnetic catheter, its location in relation to the internal structures of body tissues must be known at all times in order to ensure reliable operation. Therefore, future work should involve establishing techniques on precise localization of a magnetic catheter in clinically relevant setting. Currently used techniques, which involve medical imaging modalities, are sufficient for only coarse, low-bandwidth tracking, and suffer from low signal-to-noise ratio. Research on sensor fusion based on multi-modal tracking or development of entirely new modalities could improve this. In particular, the use of Fiber-Bragg Grating (FBG) for shape sensing of continuum robots can be regarded as a promising technology, allowing high-bandwidth feedback necessary for stable and precise control, rejecting unpredictable environmental disturbances [166,245]. Additionally, miniaturized cameras can be employed both for visual localization, as well as mapping of the environment [246].

- The subject of closed-loop control of general continuum robots has evolved in recent years, producing techniques which take into account the dynamics of the robot, thus allowing for precise position and force control [196, 247]. These techniques could potentially be translated into the subject of magnetic catheters, thereby vastly improving their behaviour in vivo and enabling fast operation in a wide range of clinically-relevant tasks. Of particular interest is the compliance/force control, which would allow a single manipulator to change its deformation characteristics, facilitating both the safe navigation to the target site, as well as the contact with body tissues [209]. Miniaturized, haptic and FBG-based sensors can be used in conjunction with force control, enabling the catheter to sense the interaction forces acting on its body and map the geometry of its environment [248, 249].

As a consequence of these developments, medical engineers of the future will gain valuable know-how which could be exploited to develop reliable devices for a wide range of possible surgical applications. The range of technologies available within the field is expected to surge, offering new possibilities both in terms of design as well as automation of magnetic catheters. Furthermore, as majority of these developments are transferable to another classes of continuum devices, research on magnetic catheters ultimately benefits continuum robotics as a whole, in applications well outside the medical domain.

\subsubsection{Novel Applications}

Ultimately, surgical robotics is intended to provide new means of assisting clinicians in effective medical treatment. Therefore, a large part of research related to magnetic catheters is focused on current medical practice. This approach, although certainly valuable and practical, restricts the potential applications of catheters 
by assuming that contemporary procedures require gradual improvement instead of a radical change.

In the same time, the larger domain of biomedical engineering seeks to define the clinicians of tomorrow. New fields of tissue engineering and immunoengieering give promise of significant expansion of the state of the art within medical practice [250,251]. Both of these domains can provide means to develop robotic tools, which realise surgical procedures by actively engaging human biology. Magneticallyactuated catheters can serve as valuable platforms enabling in vivo deployment of novel biomedical technologies, paving their way to clinical practice.

- Tissue engineering employs techniques derived from biochemistry and materials science. Its ultimate goal is to develop functional methods allowing for repairing or replacement of damaged tissues within the human body, thus enabling regenerative procedures. The principal tools used by tissue engineering are scaffolds interacting with living cells and driving their behaviour towards formation of new functional tissue. Most of current TE research involves in vitro studies in controlled environments in search for techniques allowing for successful control over tissue-related processes. However, before viable techniques can be applied in clinical practice, methods of applying them in vivo must be developed. The potential of magneticallyactuate catheters for precise navigation in deeply seated regions of human body can be exploited in the future, providing means to deploy tissue engineering constructs within the body. Furthermore, magnetic materials could be incorporated into the constructs themselves, enabling their assembly and reconfiguration in situ by means of magnetic manipulation. Ultimately, these advancements can pave the way towards minimally-invasive regenerative procedures.

- Immunoengineering is another rapidly developing field seeking to exploit biological processes in a controlled manner for the purpose of more effective treatment. By using novel materials and pharmaceuticals, research in immunoengineering seeks to modulate the natural defence mechanisms of the human body to effectively fight diseases such as cancer. The paradigms of immunoengineering stand in stark opposition to surgical robotics in its current form. Contemporary surgical robots are developed with the intentions of facilitating inherently iatrogenic interventions, disregarding the immune response or treating it as an obstacle (e.g. macrophages damaging microrobotic agents) [252]. By shifting towards immunoactive designs, future surgical robots could potentially engage the natural capabilities of the body to self-regenerate. Flexible catheters delivering localized immunoengieered functionalities could be used to execute surgical procedures not by mechanical means, but by modulation of the immune system. Even though it remains an exotic and remote prospect, such robots could potentially translate the technique of immunosurgery - which is currently applicable to a handful of stem cells on a Petri dish - in vivo [253]. This way the iatrogenic trauma incurred on the patient during surgical treatment could be completely removed.

By exploiting the novel methods of biomedical engineering, catheters and other 
surgical instruments could potentially evoke controlled biological responses of the human body. In order to achieve that, they will be required to gain new layers of sophistication, utilizing (the now relevant as the state of the art) magnetic actuation merely as a mean of moving objects around. A possible path to bioactive surgical robots leads through medical microrobotic technology, which seeks to utilize micro- and nano-robotic agents to engage cells and tissues of human body $[228,254]$. As we demonstrate in Chapters 6 and 7, magnetic catheters can serve as reliable platforms to deploy and to locally control such agents in clinically-relevant conditions. Ironically, flexible instruments and microrobotic agents, the two classes of devices once considered separate and competing, may in the future become the two constituent and inseparable elements of the same bioactive medical tools [18]. 


\section{Bibliography}

[1] J. Thorwald, The century of the surgeon. New York: Pantheon, 1957, vol. 523.

[2] R. Porter et al., The Cambridge history of medicine. Cambridge University Press, 2006.

[3] J. Eig, The Birth of the Pill: How Four Crusaders Reinvented Sex and Launched a Revolution. W. W. Norton, 2014.

[4] A. Gawande, Being mortal: Medicine and what matters in the end. Metropolitan Books, 2014.

[5] W. G. Bradley, "History of medical imaging," Proceedings of the American Philosophical Society, vol. 152, no. 3, pp. 349-361, 2008.

[6] A. M. Keeler and T. R. Flotte, "Recombinant adeno-associated virus gene therapy in light of luxturna (and zolgensma and glybera): where are we, and how did we get here?" Annual Review of Virology, vol. 6, pp. 601-621, 2019.

[7] C. Friedman, G. Hripcsak, S. B. Johnson, J. J. Cimino, and P. D. Clayton, "A generalized relational schema for an integrated clinical patient database," in Proceedings of the Annual Symposium on Computer Application in Medical Care, 1990, p. 335.

[8] C. B. Wilson, "Adoption of new surgical technology," British Medical Journal, vol. 332, no. 7533, pp. 112-114, 2006.

[9] J. Wickham, "The new surgery." British Medical Journal (Clinical research ed.), vol. 295, no. 6613, p. 1581, 1987.

[10] M. M. Siddiqui, S. Rais-Bahrami, B. Turkbey, A. K. George, J. Rothwax, N. Shakir, C. Okoro, D. Raskolnikov, H. L. Parnes, W. M. Linehan et al., "Comparison of MR/ultrasound fusion-guided biopsy with ultrasoundguided biopsy for the diagnosis of prostate cancer," Journal of American Medical Association, vol. 313, no. 4, pp. 390-397, 2015.

[11] D. D. Bensard, R. C. McIntyre Jr, B. J. Waring, and J. S. Simon, "Comparison of video thoracoscopic lung biopsy to open lung biopsy in the diagnosis of interstitial lung disease," Chest, vol. 103, no. 3, pp. 765-770, 1993. 
[12] W. M. Jackman, X. Wang, K. J. Friday, C. A. Roman, K. P. Moulton, K. J. Beckman, J. H. McClelland, N. Twidale, H. A. Hazlitt, M. I. Prior et al., "Catheter ablation of accessory atrioventricular pathways (wolffparkinson-white syndrome) by radiofrequency current," New England Journal of Medicine, vol. 324, no. 23, pp. 1605-1611, 1991.

[13] J. P. McGahan and G. D. Dodd III, "Radiofrequency ablation of the liver: current status," American Journal of Roentgenology, vol. 176, no. 1, pp. 3-16, 2001.

[14] L. Leksell, "Stereotactic radiosurgery." Journal of Neurology, Neurosurgery \& Psychiatry, vol. 46, no. 9, pp. 797-803, 1983.

[15] D. A. Larson, J. C. Flickinger, and J. S. Loeffler, "The radiobiology of radiosurgery," International Journal of Radiation Oncology, Biology, Physics, vol. 25, no. 3, pp. 557-561, 1993.

[16] S. J. Spaner and G. L. Warnock, "A brief history of endoscopy, laparoscopy, and laparoscopic surgery," Journal of Laparoendoscopic \& Advanced Surgical Techniques, vol. 7, no. 6, pp. 369-373, 1997.

[17] S. Sauerland, F. Agresta, R. Bergamaschi, G. Borzellino, A. Budzynski, G. Champault, A. Fingerhut, A. Isla, M. Johansson, P. Lundorff et al., "Laparoscopy for abdominal emergencies," Surgical Endoscopy and Other Interventional Techniques, vol. 20, no. 1, pp. 14-29, 2006.

[18] P. Gomes, "Surgical robotics: Reviewing the past, analysing the present, imagining the future," Robotics and Computer-Integrated Manufacturing, vol. 27, no. 2, pp. 261-266, 2011.

[19] P. L. Gildenberg, "The history of stereotactic neurosurgery," Neurosurgery Clinics of North America, vol. 1, no. 4, pp. 765-780, 1990.

[20] Y. S. Kwoh, J. Hou, E. A. Jonckheere, and S. Hayati, "A robot with improved absolute positioning accuracy for ct guided stereotactic brain surgery," IEEE Transactions on Biomedical Engineering, vol. 35, no. 2, pp. 153-160, 1988.

[21] S. Harris, F. Arambula-Cosio, Q. Mei, R. Hibberd, B. Davies, J. Wickham, M. Nathan, and B. Kundu, "The probot - an active robot for prostate resection," Proceedings of the Institution of Mechanical Engineers, Part H: Journal of Engineering in Medicine, vol. 211, no. 4, pp. 317-325, 1997.

[22] M. H. L. Liow, P. L. Chin, H. N. Pang, D. K.-J. Tay, and S.-J. Yeo, "THINK surgical TSolution-Oneß (Robodoc) total knee arthroplasty," SICOT-J, vol. 3, 2017.

[23] G. H. Ballantyne, "Robotic surgery, telerobotic surgery, telepresence, and telementoring," Surgical Endoscopy and Other Interventional Techniques, vol. 16, no. 10, pp. 1389-1402, 2002.

[24] T. Haidegger, J. Sándor, and Z. Benyó, "Surgery in space: the future of robotic telesurgery," Surgical Endoscopy, vol. 25, no. 3, pp. 681-690, 2011. 
[25] N. G. Hockstein, C. Gourin, R. Faust, and D. J. Terris, "A history of robots: from science fiction to surgical robotics," Journal of Robotic Surgery, vol. 1, no. 2, pp. 113-118, 2007.

[26] K. E. Lee, J. Rao, and Y.-K. Youn, "Endoscopic thyroidectomy with the da vinci robot system using the bilateral axillary breast approach (baba) technique: our initial experience," Surgical Laparoscopy Endoscopy \& Percutaneous Techniques, vol. 19, no. 3, pp. e71-e75, 2009.

[27] R. Shiroki, N. Fukami, K. Fukaya, M. Kusaka, T. Natsume, T. Ichihara, and H. Toyama, "Robot-assisted partial nephrectomy: Superiority over laparoscopic partial nephrectomy," International Journal of Urology, vol. 23, no. 2, pp. 122-131, 2016.

[28] W. W. Kim, J. S. Kim, S. M. Hur, S. H. Kim, S.-K. Lee, J. H. Choi, S. Kim, J. E. Lee, J.-H. Kim, S. J. Nam et al., "Is robotic surgery superior to endoscopic and open surgeries in thyroid cancer?" World Journal of Surgery, vol. 35, no. 4, pp. 779-784, 2011.

[29] Z. Liu, X. Li, S. Tian, T. Zhu, Y. Yao, and Y. Tao, "Superiority of robotic surgery for cervical cancer in comparison with traditional approaches: A systematic review and meta-analysis," International Journal of Surgery, vol. 40, pp. 145-154, 2017.

[30] M. Daouadi, A. H. Zureikat, M. S. Zenati, H. Choudry, A. Tsung, D. L. Bartlett, S. J. Hughes, K. K. Lee, A. J. Moser, and H. J. Zeh, "Robotassisted minimally invasive distal pancreatectomy is superior to the laparoscopic technique," Annals of Surgery, vol. 257, no. 1, pp. 128-132, 2013.

[31] G. Turchetti, I. Palla, F. Pierotti, and A. Cuschieri, "Economic evaluation of da vinci-assisted robotic surgery: a systematic review," Surgical Endoscopy, vol. 26, no. 3, pp. 598-606, 2012.

[32] G. J. Vrooijink, A. Denasi, J. G. Grandjean, and S. Misra, "Model predictive control of a robotically actuated delivery sheath for beating heart compensation," International Journal of Robotics Research, vol. 36, no. 2, pp. 193-209, 2017.

[33] F. Khan, A. Donder, S. Galvan, F. R. y Baena, and S. Misra, "Pose measurement of flexible medical instruments using fiber bragg gratings in multi-core fiber," IEEE Sensors Journal.

[34] P. E. Dupont, J. Lock, B. Itkowitz, and E. Butler, "Design and control of concentric-tube robots," IEEE Transactions on Robotics, vol. 26, no. 2, pp. 209-225, 2009.

[35] E. J. Butler, R. Hammond-Oakley, S. Chawarski, A. H. Gosline, P. Codd, T. Anor, J. R. Madsen, P. E. Dupont, and J. Lock, "Robotic neuroemdoscope with concentric tube augmentation," in 2012 IEEE/RSJ International Conference on Intelligent Robots and Systems. IEEE, 2012, pp. 2941-2946. 
[36] S. Misra, Surgical robotics: the happy accident in my life, 2018.

[37] C. Bergeles and G.-Z. Yang, "From passive tool holders to microsurgeons: safer, smaller, smarter surgical robots," IEEE Transactions on Biomedical Engineering, vol. 61, no. 5, pp. 1565-1576, 2013.

[38] G. Robinson and J. B. C. Davies, "Continuum robots-a state of the art," in Proceedings of the IEEE International Conference on Robotics and Automation (ICRA), vol. 4, 1999, pp. 2849-2854.

[39] C. Majidi, "Soft robotics: a perspective - - current trends and prospects for the future," Soft Robotics, vol. 1, no. 1, pp. 5-11, 2014.

[40] V. K. Venkiteswaran, J. Sikorski, and S. Misra, "Shape and contact force estimation of continuum manipulators using pseudo rigid body models," Mechanism and Machine Theory, vol. 139.

[41] J. Burgner-Kahrs, D. C. Rucker, and H. Choset, "Continuum Robots for Medical Applications: A Survey," IEEE Transactions on Robotics, vol. 31, no. 6, pp. 1261-1280, 2015.

[42] D. Gao, Y. Lei, and H. Zheng, "Needle steering for robot-assisted insertion into soft tissue: A survey," Chinese Journal of Mechanical Engineering, vol. 25, no. 4, pp. 629-638, 2012.

[43] N. Abolhassani, R. Patel, and M. Moallem, "Needle insertion into soft tissue: A survey," Medical Engineering \& Physics, vol. 29, no. 4, pp. 413-431, 2007.

[44] M. Azizian, M. Khoshnam, N. Najmaei, and R. V. Patel, "Visual servoing in medical robotics: a survey. part i: endoscopic and direct vision imagingtechniques and applications," The International Journal of Medical Robotics and Computer Assisted Surgery, vol. 10, no. 3, pp. 263-274, 2014.

[45] I. Elgezua, Y. Kobayashi, and M. G. Fujie, "Survey on current state-ofthe-art in needle insertion robots: Open challenges for application in real surgery," Procedia CIRP, vol. 5, pp. 94-99, 2013.

[46] H. B. Gilbert, D. C. Rucker, and R. J. Webster III, "Concentric tube robots: The state of the art and future directions," in Robotics Research. Springer, 2016, pp. 253-269.

[47] A. Ali, A. Sakes, E. A. Arkenbout, P. Henselmans, R. van Starkenburg, T. Szili-Torok, and P. Breedveld, "Catheter steering in interventional cardiology: Mechanical analysis and novel solution," Proceedings of the Institution of Mechanical Engineers, Part H: Journal of Engineering in Medicine, vol. 233, no. 12, pp. 1207-1218, 2019.

[48] K. H. Polderman and A. R. Girbes, "Central venous catheter use," Intensive Care Medicine, vol. 28, no. 1, pp. 1-17, 2002. 
[49] M. Zanchetta, G. Rigatelli, L. Pedon, M. Zennaro, E. Onorato, and P. Maiolino, "Intracardiac echocardiography during catheter-based procedures: Ultrasound system, examination technique, and image presentation," Echocardiography, vol. 19, no. 6, pp. 501-507, 2002.

[50] F. K. Wacker, J. Boese-Landgraf, A. Wagner, D. Albrecht, K.-J. Wolf, and F. Fobbe, "Minimally invasive catheter implantation for regional chemotherapy of the liver: a new percutaneous transsubclavian approach," Cardiovascular and Interventional Radiology, vol. 20, no. 2, pp. 128-132, 1997.

[51] P. Schneider, Endovascular skills: guidewire and catheter skills for endovascular surgery. CRC press, 2019.

[52] R. L. Mueller and T. A. Sanborn, "The history of interventional cardiology: cardiac catheterization, angioplasty, and related interventions," American Heart Journal, vol. 129, no. 1, pp. 146-172, 1995.

[53] V. A. Aletich, G. M. Debrun, M. Misra, F. Charbel, and J. I. Ausman, "The remodeling technique of balloon-assisted guglielmi detachable coil placement in wide-necked aneurysms: experience at the university of illinois at chicago," Journal of Neurosurgery, vol. 93, no. 3, pp. 388-396, 2000.

[54] S. O'neill, R. Greenberg, F. Haddad, T. Resch, J. Sereika, and E. Katz, “A prospective analysis of fenestrated endovascular grafting: intermediate-term outcomes," European Journal of Vascular and Endovascular Surgery, vol. 32, no. 2, pp. 115-123, 2006.

[55] R. Greenhalgh and E. The, "Comparison of endovascular aneurysm repair with open repair in patients with abdominal aortic aneurysm (evar trial 1), 30-day operative mortality results: randomised controlled trial," The Lancet, vol. 364, no. 9437, pp. 843-848, 2004.

[56] R. A. Byrne, G. W. Stone, J. Ormiston, and A. Kastrati, "Coronary balloon angioplasty, stents, and scaffolds," The Lancet, vol. 390, no. 10096, pp. 781792, 2017.

[57] M. Brueck, D. Bandorski, W. Kramer, M. Wieczorek, R. Höltgen, and H. Tillmanns, "A randomized comparison of transradial versus transfemoral approach for coronary angiography and angioplasty," JACC: Cardiovascular Interventions, vol. 2, no. 11, pp. 1047-1054, 2009.

[58] E. J. Benjamin, S. S. Virani, C. W. Callaway, A. M. Chamberlain, A. R. Chang, S. Cheng, S. E. Chiuve, M. Cushman, F. N. Delling, R. Deo et al., "Heart disease and stroke statistics - 2018 update: a report from the american heart association," Circulation, 2018.

[59] D. J. Barron and R. D. Vanderlaan, "Predicting the future: Tetralogy of fallot will be primarily treated with catheter based intervention within two decades. surgeon's perspective," Pediatric Cardiology, pp. 1-7, 2020. 
[60] R. Roelz, V. A. Coenen, C. Scheiwe, W.-D. Niesen, K. Egger, I. Csok, R. Kraeutle, R. Jabbarli, H. Urbach, and P. C. Reinacher, "Stereotactic catheter ventriculocisternostomy for clearance of subarachnoid hemorrhage: a matched cohort study," Stroke, vol. 48, no. 10, pp. 2704-2709, 2017.

[61] O. C. Mora, P. Zanne, L. Zorn, F. Nageotte, N. Zulina, S. Gravelyn, P. Montgomery, M. De Mathelin, B. Dallemagne, and M. J. Gora, "Steerable oct catheter for real-time assistance during teleoperated endoscopic treatment of colorectal cancer," Biomedical Optics Express, vol. 11, no. 3, pp. 1231$1243,2020$.

[62] R. K. Greenberg, D. Clair, S. Srivastava, G. Bhandari, A. Turc, J. Hampton, M. Popa, R. Green, and K. Ouriel, "Should patients with challenging anatomy be offered endovascular aneurysm repair?" Journal of Vascular Surgery, vol. 38, no. 5, pp. 990-996, 2003.

[63] P. W. Stather, J. B. Wild, R. D. Sayers, M. J. Bown, and E. Choke, "Endovascular aortic aneurysm repair in patients with hostile neck anatomy," Journal of Endovascular Therapy, vol. 20, no. 5, pp. 623-637, 2013.

[64] N. Piazza, R. Lange, G. Martucci, and P. W. Serruys, "Patient selection for transcatheter aortic valve implantation: patient risk profile and anatomical selection criteria," Archives of Cardiovascular Diseases, vol. 105, no. 3, pp. 165-173, 2012.

[65] A. Ali, D. H. Plettenburg, and P. Breedveld, "Steerable Catheters in Cardiology: Classifying Steerability and Assessing Future Challenges," IEEE Transactions on Biomedical Engineering, vol. 63, no. 4, pp. 679-693, 2016.

[66] H.-U. Laasch, A. Tringali, L. Wilbraham, A. Marriott, R. England, M. Mutignani, V. Perri, G. Costamagna, and D. Martin, "Comparison of standard and steerable catheters for bile duct cannulation in ercp," Endoscopy, vol. 35, no. 08, pp. 669-674, 2003.

[67] I. T. Dairywala, P. Li, Z. Liu, D. Bowie, S. R. Stewart, A.-A. Bayoumy, T. H. Murthy, and M. A. Vannan, "Catheter-based interventions guided solely by a new phased-array intracardiac imaging catheter: In vivo experimental studies," Journal of the American Society of Echocardiography, vol. 15, no. 2, pp. 150-158, 2002.

[68] C. Piorkowski, C. Eitel, S. Rolf, K. Bode, P. Sommer, T. Gaspar, S. Kircher, U. Wetzel, A. S. Parwani, L.-H. Boldt et al., "Steerable versus nonsteerable sheath technology in atrial fibrillation ablation a prospective, randomized study," Circulation: Arrhythmia and Electrophysiology, vol. 4, no. 2, pp. 157-165, 2011.

[69] R. Cappato, H. Calkins, S.-A. Chen, W. Davies, Y. Iesaka, J. Kalman, Y.-H. Kim, G. Klein, D. Packer, and A. Skanes, "Worldwide survey on the methods, efficacy, and safety of catheter ablation for human atrial fibrillation," Circulation, vol. 111, no. 9, pp. 1100-1105, 2005. 
[70] P. M. Loschak, A. Degirmenci, C. M. Tschabrunn, E. Anter, and R. D. Howe, "Automatically steering cardiac catheters in vivo with respiratory motion compensation," The International Journal of Robotics Research, vol. 39, no. 5, pp. 586-597, 2020.

[71] G. A. Antoniou, C. V. Riga, E. K. Mayer, N. J. Cheshire, and C. D. Bicknell, "Clinical applications of robotic technology in vascular and endovascular surgery," Journal of Vascular Surgery, vol. 53, no. 2, pp. 493-499, 2011.

[72] H. Rafii-Tari, C. J. Payne, and G.-Z. Yang, "Current and emerging robotassisted endovascular catheterization technologies: a review," Ann. Biomed. Eng., vol. 42, no. 4, pp. 697-715, 2014.

[73] S. A. Menaker, S. S. Shah, B. M. Snelling, S. Sur, R. M. Starke, and E. C. Peterson, "Current applications and future perspectives of robotics in cerebrovascular and endovascular neurosurgery," Journal of Neurointerventional Surgery, vol. 10, no. 1, pp. 78-82, 2018.

[74] R. J. Roesthuis and S. Misra, "Steering of Multisegment Continuum Manipulators Using Rigid-Link Modeling and FBG-Based Shape Sensing," IEEE Transactions on Robotics, vol. 32, no. 2, pp. 372-382, 2016.

[75] W. Saliba, V. Y. Reddy, O. Wazni, J. E. Cummings, J. D. Burkhardt, M. Haissaguerre, J. Kautzner, P. Peichl, P. Neuzil, V. Schibgilla et al., "Atrial fibrillation ablation using a robotic catheter remote control system: initial human experience and long-term follow-up results," Journal of the American College of Cardiology, vol. 51, no. 25, pp. 2407-2411, 2008.

[76] A. Rillig, B. Schmidt, L. Di Biase, T. Lin, L. Scholz, C. H. Heeger, A. Metzner, D. Steven, P. Wohlmuth, S. Willems et al., "Manual versus robotic catheter ablation for the treatment of atrial fibrillation: the man and machine trial," JACC: Clinical Electrophysiology, vol. 3, no. 8, pp. 875-883, 2017.

[77] P. Kanagaratnam, M. Koa-Wing, D. T. Wallace, A. S. Goldenberg, N. S. Peters, and D. W. Davies, "Experience of robotic catheter ablation in humans using a novel remotely steerable catheter sheath," Journal of Interventional Cardiac Electrophysiology, vol. 21, no. 1, pp. 19-26, 2008.

[78] D. Thomas, E. P. Scholz, P. A. Schweizer, H. A. Katus, and R. Becker, "Initial experience with robotic navigation for catheter ablation of paroxysmal and persistent atrial fibrillation," Journal of Electrocardiology, vol. 45, no. 2, pp. 95-101, 2012.

[79] C. V. Riga, N. J. Cheshire, M. S. Hamady, and C. D. Bicknell, "The role of robotic endovascular catheters in fenestrated stent grafting," Journal of Vascular Surgery, vol. 51, no. 4, pp. 810-820, 2010.

[80] B. S. Peters, P. R. Armijo, C. Krause, S. A. Choudhury, and D. Oleynikov, "Review of emerging surgical robotic technology," Surgical Endoscopy, vol. 32, no. 4, pp. 1636-1655, 2018. 
[81] G. J. Vrooijink, T. Ellenbroek, P. Breedveld, J. G. Grandjean, and S. Misra, "A preliminary study on using a Robotically-Actuated Delivery Sheath (RADS) for transapical aortic valve implantation," Proceedings of 2014 IEEE International Conference on Robotics and Automation (ICRA), pp. 43804386, 2014.

[82] P. M. Loschak, L. J. Brattain, and R. D. Howe, "Automated pointing of cardiac imaging catheters," in Proceedings of the IEEE International Conference on Robotics and Automation (ICRA), 2013, pp. 5794-5799.

[83] W. Zhao and J. P. Desai, "Towards the development of a new tendon-driven minimally invasive neurosurgical intracranial robot," in $A S M E 2014 D y$ namic Systems and Control Conference, 2014.

[84] J. P. Desai, M. Ho, J. M. Simard, and R. Gullapalli, "Minimally invasive neurosurgical intracranial robot system and method," Aug. 22 2013, US Patent App. 13/763,284.

[85] D. B. Camarillo, C. F. Milne, C. R. Carlson, M. R. Zinn, and J. K. Salisbury, "Mechanics modeling of tendon-driven continuum manipulators," IEEE Transactions on Robotics, vol. 24, no. 6, pp. 1262-1273, 2008.

[86] T. Kimura, S. Takatsuki, A. Oishi, M. Negishi, S. Kashimura, Y. Katsumata, T. Nishiyama, N. Nishiyama, Y. Tanimoto, and Y. Aizawa, "Operatorblinded contact force monitoring during pulmonary vein isolation using conventional and steerable sheaths," International Journal Cardiol., vol. 177, no. 3, pp. 970-976, 2014.

[87] G. Subramani and M. R. Zinn, "Tackling friction-an analytical modeling approach to understanding friction in single tendon driven continuum manipulators," in Proceedings of 2015 IEEE International Conference on Robotics and Automation (ICRA), 2015, pp. 610-617.

[88] K. Ikuta, H. Ichikawa, and K. Suzuki, "Safety-active catheter with multiplesegments driven by micro-hydraulic actuators," in International Conference on Medical Image Computing and Computer-Assisted Intervention. Springer, 2002, pp. 182-191.

[89] Y. Haga, Y. Muyari, T. Mineta, T. Matsunaga, H. Akahori, and M. Esashi, "Small diameter hydraulic active bending catheter using laser processed super elastic alloy and silicone rubber tube," in 2005 3rd IEEE/EMBS Special Topic Conference on Microtechnology in Medicine and Biology, 2005, pp. 245-248.

[90] R. R. Miles, D. L. Schumann, M. A. Pearson, and D. Hilken, "Steerable catheter with distending lumen-actuated curling catheter tip," Jan. 14 2010, US Patent App. 12/502,097.

[91] E. Ayvali, C.-P. Liang, M. Ho, Y. Chen, and J. P. Desai, "Towards a discretely actuated steerable cannula for diagnostic and therapeutic procedures," The International Journal of Robotics Research, vol. 31, no. 5, pp. 588-603, 2012. 
[92] M. Langelaar and F. van Keulen, "Modeling of a shape memory alloy active catheter," in 45th AIAA/ASME/ASCE/AHS/ASC Structures, Structural Dynamics 6 Materials Conference, 2004, p. 1653.

[93] R. E. Goldman, A. Bajo, and N. Simaan, "Compliant motion control for multisegment continuum robots with actuation force sensing," IEEE Transactions on Robotics, vol. 30, no. 4, pp. 890-902, 2014.

[94] T. da Veiga, J. H. Chandler, P. Lloyd, G. Pittiglio, N. J. Wilkinson, A. K. Hoshiar, R. A. Harris, and P. Valdastri, "Challenges of continuum robots in clinical context: a review." Progress in Biomedical Engineering, vol. 2, no. 3, 2020 .

[95] S. L. Charreyron, B. Zeydan, and B. J. Nelson, "Shared control of a magnetic microcatheter for vitreoretinal targeted drug delivery," in 2017 IEEE International Conference on Robotics and Automation (ICRA), May 2017, pp. 4843-4848.

[96] A. J. Petruska, J. Edelmann, and B. J. Nelson, "Model-based calibration for magnetic manipulation," IEEE Transactions on Magnetics, vol. 53, no. 7, pp. 1-6, 2017.

[97] P. R. Lloyd, T. Da Veiga, A. Attanasio, N. Marahrens, J. H. Chandler, P. Valdastri et al., "A learnt approach for the design of magnetically actuated shape forming soft tentacle robots," IEEE Robotics and Automation Letters, 2020.

[98] D. J. Griffiths and C. Inglefield, Introduction to Electrodynamics, 3rd ed. Upper Saddle River, NJ: Prentice Hall, 2005.

[99] A. J. Petruska and B. J. Nelson, "Minimum bounds on the number of electromagnets required for remote magnetic manipulation," IEEE Transactions on Robotics, vol. 31, no. 3, pp. 714-722, 2015.

[100] A. J. Petruska and J. J. Abbott, "Optimal permanent-magnet geometries for dipole field approximation," IEEE Transactions on Magnetics, vol. 49, no. 2, pp. 811-819, 2013.

[101] M. P. Kummer, J. J. Abbott, B. E. Kratochvil, R. Borer, A. Sengul, and B. J. Nelson, "OctoMag: An electromagnetic system for 5-DOF wireless micromanipulation," IEEE Transactions on Robotics, vol. 26, no. 6, pp. 1006-1017, 2010.

[102] J. J. Abbott, E. Diller, and A. J. Petruska, "Magnetic methods in robotics," Annual Review of Control, Robotics, and Autonomous Systems, vol. 3, 2019.

[103] T. Xu, J. Yu, X. Yan, H. Choi, and L. Zhang, "Magnetic actuation based motion control for microrobots: An overview," Micromachines, vol. 6, no. 9, pp. 1346-1364, 2015. 
[104] M. Sitti, H. Ceylan, W. Hu, J. Giltinan, M. Turan, S. Yim, and E. Diller, "Biomedical applications of untethered mobile milli/microrobots," Proceedings of the IEEE, vol. 103, no. 2, pp. 205-224, 2015.

[105] B. J. Nelson, I. K. Kaliakatsos, and J. J. Abbott, "Microrobots for minimally invasive medicine," Annual Review of Biomedical Engineering, vol. 12, pp. 55-85, 2010.

[106] M. P. Kummer, J. J. Abbott, B. E. Kratochvil, R. Borer, A. Sengul, and B. J. Nelson, "Octomag: An electromagnetic system for 5-DOF wireless micromanipulation," IEEE Transactions on Robotics, vol. 26, no. 6, pp. 1006-1017, 2010 .

[107] A. Pourkand and J. J. Abbott, "A critical analysis of eight-electromagnet manipulation systems: The role of electromagnet configuration on strength, isotropy, and access," IEEE Robotics and Automation Letters, vol. 3, no. 4, pp. 2957-2962, 2018.

[108] F. Ongaro, S. Pane, S. Scheggi, and S. Misra, "Design of an electromagnetic setup for independent three-dimensional control of pairs of identical and nonidentical microrobots," IEEE Transactions on Robotics, vol. 35, no. 1, pp. 174-183, 2018.

[109] C. Di Natali, M. Beccani, N. Simaan, and P. Valdastri, "Jacobian-Based Iterative Method for Magnetic Localization in Robotic Capsule Endoscopy," IEEE Transactions on Robotics, vol. 32, no. 2, pp. 327-338, 2016.

[110] P. R. Slawinski, A. Z. Taddese, K. B. Musto, K. L. Obstein, and P. Valdastri, "Autonomous retroflexion of a magnetic flexible endoscope," IEEE Robotics and Automation Letters, vol. 2, no. 3, pp. 1352-1359, 2017.

[111] G. Pittiglio, L. Barducci, J. W. Martin, J. C. Norton, C. A. Avizzano, K. L. Obstein, and P. Valdastri, "Magnetic levitation for soft-tethered capsule colonoscopy actuated with a single permanent magnet: a dynamic control approach," IEEE Robotics and Automation Letters, vol. 4, no. 2, pp. 12241231, 2019.

[112] A. J. Petruska, F. Ruetz, A. Hong, L. Regli, O. Sürücü, A. Zemmar, and B. J. Nelson, "Magnetic needle guidance for neurosurgery: Initial design and proof of concept," in 2016 IEEE International Conference on Robotics and Automation (ICRA). IEEE, 2016, pp. 4392-4397.

[113] M. Ilami, R. J. Ahmed, A. Petras, B. Beigzadeh, and H. Marvi, "Magnetic needle steering in soft phantom tissue," Scientific Reports, vol. 10, no. 1, pp. $1-11,2020$.

[114] H. Tillander, "Magnetic guidance of a catheter with articulated steel tip," Acta radiologica, vol. 35, no. 1, pp. 62-64, 1951.

[115] C. M. Heunis, Y. P. Wotte, J. Sikorski, G. P. Furtado, and S. Misra, "The ARMM system - autonomous steering of magnetically-actuated catheters: 
Towards endovascular applications," IEEE Robotics and Automation Letters, vol. 5, no. 2, pp. 705-712, 2020.

[116] J. Edelmann, A. J. Petruska, and B. J. Nelson, "Magnetic control of continuum devices," International Journal of Robotics Research, vol. 36, no. 1, pp. 68-85, 2017.

[117] V. N. T. Le, N. H. Nguyen, K. Alameh, R. Weerasooriya, and P. Pratten, "Accurate modeling and positioning of a magnetically controlled catheter tip," Medical Physics, vol. 43, no. 2, pp. 650-663, 2016.

[118] C. Chautems, A. Tonazzini, D. Floreano, and B. J. Nelson, "A variable stiffness catheter controlled with an external magnetic field," in Proceedings of the 2017 IEEE/RSJ International Conference on Intelligent Robots and Systems (IROS), Sept 2017, pp. 181-186.

[119] F. Settecase, M. S. Sussman, M. W. Wilson, S. Hetts, R. L. Arenson, V. Malba, A. F. Bernhardt, W. Kucharczyk, and T. P. Roberts, "Magnetically-assisted remote control (MARC) steering of endovascular catheters for interventional MRI: a model for deflection and design implications," Medical Physics, vol. 34, no. 8, pp. 3135-3142, 2007.

[120] A. D. Losey, P. Lillaney, A. J. Martin, D. L. Cooke, M. W. Wilson, B. R. Thorne, R. S. Sincic, R. L. Arenson, M. Saeed, and S. W. Hetts, "Magnetically assisted remote-controlled endovascular catheter for interventional MR imaging: in vitro navigation at $1.5 \mathrm{~T}$ versus X-ray fluoroscopy," Radiology, vol. 271, no. 3, pp. 862-869, 2014.

[121] T. Greigarn, R. Jackson, T. Liu, and M. C. Çavuşoğlu, "Experimental validation of the pseudo-rigid-body model of the MRI-actuated catheter," in Proceedings of the 2017 IEEE International Conference on Robotics and Automation (ICRA), 2017, pp. 3600-3605.

[122] T. Liu, N. L. Poirot, D. Franson, N. Seiberlich, M. Griswold, and M. Cavusoglu, "Modeling and validation of the three dimensional deflection of an MRI-compatible magnetically-actuated steerable catheter," IEEE Transactions on Biomedical Engineering, vol. 63, no. 10, pp. 2142-2154, 2015.

[123] Stereotaxis. (2020) Niobe magnetic navigation system. [Online]. Available: http://www.stereotaxis.com/products/niobe/

[124] F. Carpi and C. Pappone, "Stereotaxis niobe $\AA$ magnetic navigation system for endocardial catheter ablation and gastrointestinal capsule endoscopy," Expert review of medical devices, vol. 6, no. 5, pp. 487-498, 2009.

[125] S. A. Virk and S. Kumar, "Remote magnetic versus manual catheter navigation for atrial fibrillation ablation: a meta-analysis," Circulation: Arrhythmia and Electrophysiology, vol. 12, no. 10, p. e007517, 2019.

[126] F. Kiemeneij, M. S. Patterson, G. Amoroso, G. Laarman, and T. Slagboom, "Use of the stereotaxis niobeß magnetic navigation system for percutaneous 
coronary intervention: Results from 350 consecutive patients," Catheterization and Cardiovascular Interventions, vol. 71, no. 4, pp. 510-516, 2008.

[127] E. I. Canales, J. Córdova, J. Escaned, and R. Hernández-Antolín, "Stereotaxis: a new approach to treating highly tortuous and angulated coronary lesions after conventional techniques have failed," Revista Espanola de Cardiologia, vol. 62, no. 8, pp. 942-944, 2009.

[128] L. Lüthje, D. Vollmann, J. Seegers, M. Dorenkamp, C. Sohns, G. Hasenfuss, and M. Zabel, "Remote magnetic versus manual catheter navigation for circumferential pulmonary vein ablation in patients with atrial fibrillation," Clinical Research in Cardiology, vol. 100, no. 11, p. 1003, 2011.

[129] R. Proietti, V. Pecoraro, L. Di Biase, A. Natale, P. Santangeli, M. Viecca, A. Sagone, A. Galli, L. Moja, and L. Tagliabue, "Remote magnetic with open-irrigated catheter vs. manual navigation for ablation of atrial fibrillation: a systematic review and meta-analysis," Europace, vol. 15, no. 9, pp. 1241-1248, 2013.

[130] (2020) Stereotaxis receives FDA clearance for Genesis Robotic Magnetic Navigation system. [Online]. Available: https://cardiacrhythmnews.com/ stereotaxis-receives-fda-clearance-for-genesis-robotic-magnetic-navigation-system/

[131] Y. Shachar, "Apparatus and method for shaped magnetic field control for catheter, guidance, control, and imaging," Sep. 27 2011, US Patent 8,027,714.

[132] M. N. Faddis, J. Chen, J. Osborn, M. Talcott, M. E. Cain, and B. D. Lindsay, "Magnetic guidance system for cardiac electrophysiology: a prospective trial of safety and efficacy in humans," J. Am. Coll. Cardiol., vol. 42, no. 11, pp. 1952-1958, 2003.

[133] C. Chautems, B. Zeydan, S. Charreyron, G. Chatzipirpiridis, S. Pane, and B. J. Nelson, "Magnetically powered microrobots: a medical revolution underway?" European Journal of Cardio-Thoracic Surgery, vol. 51, no. 3, pp. 405-407, 2017.

[134] C. Heunis, J. Sikorski, and S. Misra, "Flexible instruments for endovascular interventions: improved magnetic steering, actuation, and image-guided surgical instruments," IEEE Robotics and Automation Magazine, vol. 25, no. 3, pp. 71-82, 2018.

[135] W. Lee, J. Nam, J. Kim, E. Jung, N. Kim, and G. Jang, "Steering, tunneling, and stent delivery of a multifunctional magnetic catheter robot to treat occlusive vascular disease," IEEE Transactions on Industrial Electronics, p. Early Access, 2020.

[136] F. Ullrich, S. Schuerle, R. Pieters, A. Dishy, S. Michels, and B. J. Nelson, "Automated capsulorhexis based on a hybrid magnetic-mechanical actuation system," in Proceedings of the IEEE International Conference on Robotics and Automation (ICRA), 2014, pp. 4387-4392. 
[137] S. Jeon, A. K. Hoshiar, K. Kim, S. Lee, E. Kim, S. Lee, J.-y. Kim, B. J. Nelson, H.-J. Cha, B.-J. Yi et al., "A magnetically controlled soft microrobot steering a guidewire in a three-dimensional phantom vascular network," Soft Robotics, vol. 6, no. 1, pp. 54-68, 2019.

[138] K. M. Popek, T. Hermans, and J. J. Abbott, "First demonstration of simultaneous localization and propulsion of a magnetic capsule in a lumen using a single rotating magnet," in Proceedings of the 2017 International Conference on Robotics and Automation (ICRA), June 2017, pp. 1154-1160.

[139] L. B. Kratchman, T. L. Bruns, J. J. Abbott, and R. J. Webster III, "Guiding elastic rods with a robot-manipulated magnet for medical applications," IEEE Transactions on Robotics, vol. 33, no. 1, pp. 227-233, 2016.

[140] N. Garbin, P. Valdastri, K. L. Obstein, N. Simaan, and P. R. Slawinski, "Steerable endoscope with continuum manipulator," Jan. 16 2020, US Patent App. 16/465,621.

[141] T. Greigarn, N. L. Poirot, X. Xu, and M. C. Çavuşoğlu, "Jacobian-based task-space motion planning for MRI-actuated continuum robots," IEEE Robotics and Automation Letters, vol. 4, no. 1, pp. 145-152, 2019.

[142] I. Tunay, "Position control of catheters using magnetic fields," in Proceedings of the 2004 International Conference on Robotics and Automation (ICRA), 2004, pp. 392-397.

[143] A. Azizi, C. C. Tremblay, K. Gagné, and S. Martel, "Using the fringe field of a clinical MRI scanner enables robotic navigation of tethered instruments in deeper vascular regions," Science Robotics, vol. 4, no. 36, 2019.

[144] C. Chautems and B. J. Nelson, "The tethered magnet: Force and 5-DOF pose control for cardiac ablation," in Proceedings of the 2017 IEEE International Conference on Robotics and Automation (ICRA), 2017, pp. 4837-4842.

[145] S. L. Charreyron, E. Gabbi, Q. Boehler, M. Becker, and B. J. Nelson, "A magnetically steered endolaser probe for automated panretinal photocoagulation," IEEE Robotics and Automation Letters, vol. 4, no. 2, pp. 284-290, April 2019.

[146] D. Son, H. Gilbert, and M. Sitti, "Magnetically actuated soft capsule endoscope for fine-needle biopsy," Soft Robotics, vol. 7, no. 1, pp. 10-21, 2020.

[147] S. Jeon, J. Nam, W. Lee, and G. Jang, "Selective navigating and unclogging motions of an intravascular helical magnetic millirobot actuated by external biaxial rotating magnetic fields," IEEE/ASME Transactions on Mechatronics, vol. 22, no. 3, pp. 1456-1464, 2017.

[148] V. K. Venkiteswaran, L. F. P. Samaniego, J. Sikorski, and S. Misra, "Bioinspired terrestrial motion of magnetic soft millirobots," IEEE Robotics and automation letters, vol. 4, no. 2, pp. 1753-1759, 2019. 
[149] G. Z. Lum, Z. Ye, X. Dong, H. Marvi, O. Erin, W. Hu, and M. Sitti, "Shapeprogrammable magnetic soft matter," Proceedings of the National Academy of Sciences, vol. 113, no. 41, pp. E6007-E6015, 2016.

[150] H. Lu, M. Zhang, Y. Yang, Q. Huang, T. Fukuda, Z. Wang, and Y. Shen, "A bioinspired multilegged soft millirobot that functions in both dry and wet conditions," Nature Communications, vol. 9, no. 1, pp. 1-7, 2018.

[151] E. Diller, J. Zhuang, G. Zhan Lum, M. R. Edwards, and M. Sitti, "Continuously distributed magnetization profile for millimeter-scale elastomeric undulatory swimming," Applied Physics Letters, vol. 104, no. 17, p. 174101, 2014 .

[152] P. Wang, T. Chen, Y. Zhu, W. Zhang, S. K. Zhou, and D. Comaniciu, "Robust guidewire tracking in fluoroscopy," in Proceedings of the 2009 IEEE Conference on Computer Vision and Pattern Recognition, 2009, pp. 691-698.

[153] Y. Ma, N. Gogin, P. Cathier, R. J. Housden, G. Gijsbers, M. Cooklin, M. O'Neill, J. Gill, C. A. Rinaldi, R. Razavi et al., "Real-time x-ray fluoroscopy-based catheter detection and tracking for cardiac electrophysiology interventions," Medical pPhysics, vol. 40, no. 7, p. 071902, 2013.

[154] A. Hong, A. J. Petruska, and B. J. Nelson, "Tracking a magnetically guided catheter with a single rotating c-arm," in Proceedings of the 2015 IEEE International Conference on Robotics and Automation (ICRA), 2015, pp. 618-623.

[155] K. J. Boskma, S. Scheggi, and S. Misra, "Closed-loop control of a magnetically-actuated catheter using two-dimensional ultrasound images," Proceedings of 2016 6th IEEE RAS/EMBS International Conference on Biomedical Robotics and Biomechatronics (BioRob), pp. 61-66, 2016.

[156] D. B. Camarillo, K. E. Loewke, C. R. Carlson, and J. K. Salisbury, "Vision based 3-d shape sensing of flexible manipulators," in Proceedings of 2008 IEEE International Conference on Robotics and Automation (ICRA), 2008, pp. $2940-2947$.

[157] J. M. Croom, D. C. Rucker, J. M. Romano, and R. J. Webster, "Visual sensing of continuum robot shape using self-organizing maps," in Proceedings of the 2010 IEEE International Conference on Robotics and Automation (ICRA), 2010, pp. 4591-4596.

[158] E. J. Lobaton, J. Fu, L. G. Torres, and R. Alterovitz, "Continuous shape estimation of continuum robots using x-ray images," in Proceedings of the 2013 IEEE International Conference on Robotics and Automation. IEEE, 2013, pp. $725-732$.

[159] W.-S. Lee and T. Poston, "Rapid 3d tube reconstruction from nearby views," in Fifth International Conference in Central Europe in Computer Graphics and Visualization, 1997, pp. 262-271. 
[160] G. J. Vrooijink, M. Abayazid, and S. Misra, "Real-time three-dimensional flexible needle tracking using two-dimensional ultrasound," in Proceedings of the IEEE International Conference on Robotics and Automation (ICRA), 2013, pp. 1688-1693.

[161] B. He, S. Xu, and Z. Wang, "Research on stiffness of multibackbone continuum robot based on screw theory and euler-bernoulli beam," Mathematical Problems in Engineering, vol. 2018, 2018.

[162] D. C. Rucker, B. A. Jones, and R. J. Webster, "A geometrically exact model for externally loaded concentric-tube continuum robots," IEEE Transactions on Robotics, vol. 26, no. 5, pp. 769-780, 2010.

[163] M. Mahvash and P. E. Dupont, "Stiffness control of a continuum manipulator in contact with a soft environment," in Proceedings of the 2010 IEEE/RSJ International Conference on Intelligent Robots and Systems, 2010, pp. 863870 .

[164] S. Grazioso, G. Di Gironimo, and B. Siciliano, "A geometrically exact model for soft continuum robots: The finite element deformation space formulation," Soft Robotics, vol. 6, no. 6, pp. 790-811, 2019.

[165] M. Khoshnam and R. V. Patel, "A pseudo-rigid-body 3r model for a steerable ablation catheter," in Proceedings of the 2013 IEEE International Conference on Robotics and Automation, 2013, pp. 4427-4432.

[166] R. J. Roesthuis and S. Misra, "Steering of multisegment continuum manipulators using rigid-link modeling and FBG-based shape sensing," IEEE Transactions on Robotics, vol. 32, no. 2, pp. 372-382, 2016.

[167] V. K. Venkiteswaran and H.-J. Su, "Development of a 3-spring pseudo rigid body model of compliant joints for robotic applications," in ASME 2014 International Design Engineering Technical Conferences and Computers and Information in Engineering Conference. American Society of Mechanical Engineers Digital Collection, 2014.

[168] T. arn and M. C. Çavuşoğlu, "Task-space motion planning of MRI-actuated catheters for catheter ablation of atrial fibrillation," in Proceedings of 2014 IEEE/RSJ International Conference on Intelligent Robots and Systems, 2014, pp. 3476-3482.

[169] T. Greigarn and M. C. Çavuşoğlu, "Pseudo-rigid-body model and kinematic analysis of MRI-actuated catheters," Proceedings of the 2015 IEEE International Conference on Robotics and Automation (ICRA), pp. 2236-2243, May 2015.

[170] R. J. Webster and B. A. Jones, "Design and Kinematic Modeling of Constant Curvature Continuum Robots: A Review," The International Journal of Robotics Research, vol. 29, no. 13, pp. 1661-1683, 2010. 
[171] I. A. Gravagne and I. D. Walker, "Manipulability, force, and compliance analysis for planar continuum manipulators," IEEE Transactions on Robotics and Automation, vol. 18, no. 3, pp. 263-273, 2002.

[172] T. Kimura, S. Takatsuki, A. Oishi, M. Negishi, S. Kashimura, Y. Katsumata, T. Nishiyama, N. Nishiyama, Y. Tanimoto, and Y. Aizawa, "Operatorblinded contact force monitoring during pulmonary vein isolation using conventional and steerable sheaths," International Journal of Cardiology, vol. 177, no. 3, pp. 970-976, 2014.

[173] A. Kangarlu, R. E. Burgess, H. Zhu, T. Nakayama, R. L. Hamlin, A. M. Abduljalil, and P. M. L. Robitaille, "Cognitive, cardiac, and physiological safety studies in ultra high field magnetic resonance imaging," Magnetic resonance imaging, vol. 17, no. 10, pp. 1407-1416, 1999.

[174] F. Ullrich, S. Schuerle, R. Pieters, A. Dishy, S. Michels, and B. J. Nelson, "Automated capsulorhexis based on a hybrid magnetic-mechanical actuation system," Proceedings of 2014 IEEE International Conference on Robotics and Automation (ICRA), pp. 4387-4392, 2014.

[175] F. Carpi and C. Pappone, "Stereotaxis Niobe $\Re$ magnetic navigation system for endocardial catheter ablation and gastrointestinal capsule endoscopy," Expert Review of Medical Devices, vol. 6, no. 5, pp. 487-498, 2009.

[176] B. Véron, A. Hubert, J. Abadie, and N. Andreff, "Geometric analysis of the singularities of a magnetic manipulation system with several mobile coils," Proceedings of the 2013 IEEE/RSJ International Conference on Intelligent Robots and Systems, pp. 4996-5001, 2013.

[177] F. P. Gosselin, V. Lalande, and S. Martel, "Characterization of the deflections of a catheter steered using a magnetic resonance imaging system," Medical Physics, vol. 38, no. 9, pp. 4994-5002, 2011.

[178] A. M. Gilroy, B. R. MacPherson, L. M. Ross, J. Broman, and A. Josephson, Atlas of anatomy. Thieme Stuttgart, 2008.

[179] J. Sikorski, I. R. Dawson, A. Denasi, E. E. G. Hekman, and S. Misra, "Introducing BigMag - a novel system for 3D actuation of flexible surgical devices," Proceedings of the 2017 IEEE International Conference on Robotics and Automation (ICRA), pp. 3594-3599, 2017.

[180] S. Chiaverini, B. Siciliano, and O. Egeland, "Review of the damped leastsquares inverse kinematics with experiments on an industrial robot manipulator," IEEE Transactions on Control Systems Technology, vol. 2, no. 2, pp. 123-134, 1994.

[181] Health Quality Ontario, "Coil embolization for intracranial aneurysms: an evidence-based analysis," Ontario Health Technology Assessment Series, vol. 6, no. 1, pp. 1-114, 2006. 
[182] N. Gudino, J. Heilman, J. Derakhshan, J. L. Sunshine, J. L. Duerk, and M. A. Griswold, "Control of intravascular catheters using an array of active steering coils," Medical Physics, vol. 38, no. 7, pp. 4215-4224, 2011.

[183] S. E. Wright, A. W. Mahoney, K. M. Popek, and J. J. Abbott, "The sphericalactuator-magnet manipulator: A permanent-magnet robotic end-effector," IEEE Transactions on Robotics, vol. 33, no. 5, pp. 1013-1024, 2017.

[184] I. S. Khalil, B. E. Wissa, B. G. Salama, and S. Stramigioli, "Wireless motion control of paramagnetic microparticles using a magnetic-based robotic system with an open-configuration," in Proceedings of the 2015 International Conference on Manipulation, Manufacturing and Measurement on the Nanoscale (3M-NANO), 2015, pp. 190-196.

[185] B. Veron, A. Hubert, J. Abadie, N. Andreff, and P. Renaud, "Advocacy for multi mobile coil magnetic manipulation in active digestive endoscopy." in IEEE/RSJ 2012 International Conference on Intelligent Robots and Systems (IROS)., 2012.

[186] B. Véron, J. Abadie, A. Hubert, and N. Andreff, "Magnetic manipulation with several mobile coils towards gastrointestinal capsular endoscopy," in New Trends in Mechanism and Machine Science. Springer, 2013, pp. 681689.

[187] G. Lucarini, G. Ciuti, M. Mura, R. Rizzo, and A. Menciassi, "A new concept for magnetic capsule colonoscopy based on an electromagnetic system," International Journal of Advanced Robotic Systems, vol. 12, no. 3, p. 25, 2015 .

[188] G. Lucarini, M. Mura, G. Ciuti, R. Rizzo, and A. Menciassi, "Electromagnetic control system for capsule navigation: Novel concept for magnetic capsule maneuvering and preliminary study," Journal of Medical and Biological Engineering, vol. 35, no. 4, pp. 428-436, 2015.

[189] J. J. Abbott and B. Osting, "Optimization of coreless electromagnets to maximize field generation for magnetic manipulation systems," IEEE Magnetics Letters, vol. 8, pp. 1-4, 2017.

[190] S. Muntwyler, B. Kratochvil, B. Nelson, D. Frutiger, D. Bell, and J. Baumann, "Magnetic navigation system with soft magnetic core electromagnets for operation in the non-linear regime," 2017, US Patent 9,681,859.

[191] F. Beyeler, S. Muntwyler, Z. Nagy, C. Graetzel, M. Moser, and B. J. Nelson, "Design and calibration of a mems sensor for measuring the force and torque acting on a magnetic microrobot," Journal of Micromechanics and Microengineering, vol. 18, no. 2, p. 025004, 2007.

[192] P. S. Rahko, "Evaluation of the skin-to-heart distance in the standing adult by two-dimensional echocardiography," Journal of the American Society of Echocardiography, vol. 21, no. 6, pp. 761-764, 2008. 
[193] R. C. Dorf, The Electrical Engineering Handbook. CRC press, 1997.

[194] J. Sikorski, A. Denasi, G. Bucchi, S. Scheggi, and S. Misra, "Vision-based 3-d control of magnetically actuated catheter using BigMag - an array of mobile electromagnetic coils," IEEE/ASME Transactions on Mechatronics, vol. 24, no. 2, pp. 505-516, 2019.

[195] J. M. Tobis and I. Abudayyeh, "New devices and technology in interventional cardiology," Journal of Cardiology, vol. 65, no. 1, pp. 5-16, 2015.

[196] A. Bajo and N. Simaan, "Hybrid motion/force control of multi-backbone continuum robots," The International Journal of Robotics Besearch, vol. 35, no. 4, pp. 422-434, 2016.

[197] F. Settecase, M. S. Sussman, M. W. Wilson, S. Hetts, R. L. Arenson, V. Malba, A. F. Bernhardt, W. Kucharczyk, and T. P. L. Roberts, "Magnetically-assisted remote control (MARC) steering of endovascular catheters for interventional MRI: a model for deflection and design implications." Medical Physics, vol. 34, no. 8, pp. 3135-3142, 2007.

[198] T. Liu, N. L. Poirot, D. Franson, N. Seiberlich, M. Griswold, and M. Cavusoglu, "Modeling and Validation of the Three Dimensional Deflection of an MRI-Compatible Magnetically-Actuated Steerable Catheter," IEEE Transactions on Biomedical Engineering, vol. PP, no. 99, pp. 1-1, 2016.

[199] T. Greigarn and M. C. Çavuşoğlu, "Pseudo-rigid-body model and kinematic analysis of MRI-actuated catheters," in Proceedings of 2015 IEEE International Conference on Robotics and Automation (ICRA), 2015, pp. 22362243 .

[200] S. W. Hetts, M. Saeed, A. J. Martin, L. Evans, A. F. Bernhardt, V. Malba, F. Settecase, L. Do, E. J. Yee, and A. Losey, "Endovascular catheter for magnetic navigation under MR imaging guidance: Evaluation of safety in vivo at 1.5 T," American Journal of Neuroradiology, vol. 34, no. 11, pp. 2083-2091, 2013.

[201] B. L. Nguyen, J. L. Merino, and E. S. Gang, "Remote navigation for ablation procedures-a new step forward in the treatment of cardiac arrhythmias," European Cardiology, vol. 6, no. 3, pp. 50-56, 2010.

[202] A. Laurentini, "The visual hull concept for silhouette-based image understanding," IEEE Transactions on Pattern Analysis and Machine Intelligence, vol. 16, no. 2, pp. 150-162, 1994.

[203] A. D. Losey, P. Lillaney, A. J. Martin, D. L. Cooke, M. W. Wilson, B. R. H. Thorne, R. S. Sincic, R. L. Arenson, M. Saeed, and S. W. Hetts, "Magnetically assisted remote-controlled endovascular catheter for interventional MR imaging: in vitro navigation at $1.5 \mathrm{~T}$ versus x-ray fluoroscopy," Radiology, vol. 271, no. 3, pp. 862-869, 2014. 
[204] G. Dogangil, B. L. Davies, and F. R. Y Baena, "A review of medical robotics for minimally invasive soft tissue surgery," Proceedings of the Institution of Mechanical Engineers, Part H: Journal of Engineering in Medicine, vol. 224, no. 5, pp. 653-679, 2010.

[205] C. Di Natali, J. Buzzi, N. Garbin, M. Beccani, and P. Valdastri, "Closed-loop control of local magnetic actuation for robotic surgical instruments," IEEE Transactions on Robotics, vol. 31, no. 1, pp. 143-156, 2015.

[206] N. Giri and I. Walker, "Continuum robots and underactuated grasping," Mechanical Sciences, vol. 2, no. 1, pp. 51-58, 2011.

[207] F. Burbank, S. H. Parker, and T. J. Fogarty, "Stereotactic breast biopsy: improved tissue harvesting with the mammotome." The American Surgeon, vol. 62, no. 9, pp. 738-744, 1996.

[208] J. J. Abbott, E. Diller, and A. J. Petruska, "Magnetic methods in robotics," Annual Review of Control, Robotics, and Autonomous Systems, vol. 3, pp. 57-90.

[209] C. Chautems, A. Tonazzini, D. Floreano, and B. J. Nelson, "A variable stiffness catheter controlled with an external magnetic field," in Proceedings of the 2017 IEEE/RSJ International Conference on Intelligent Robots and Systems (IROS), 2017, pp. 181-186.

[210] J. Sikorski, E. S. Rutting, and S. Misra, "Grasping using magneticallyactuated tentacle catheter: A proof-of-concept study," in Proceedings of the 2018 7th IEEE International Conference on Biomedical Robotics and Biomechatronics (Biorob), 2018, pp. 609-614.

[211] X. Chen, B. Jang, D. Ahmed, C. Hu, C. D. Marco, M. Hoop, F. Mushtaq, B. J. Nelson, and S. Pane, "Small-scale machines driven by external power sources," Advanced Materials, 2018.

[212] A. Vikram Singh and M. Sitti, "Targeted drug delivery and imaging using mobile milli/microrobots: A promising future towards theranostic pharmaceutical design," Current Pharmaceutical Design, vol. 22, no. 11, pp. 14181428, 2016.

[213] F. Ongaro, S. Scheggi, C. Yoon, F. Van den Brink, S. H. Oh, D. H. Gracias, and S. Misra, "Autonomous planning and control of soft untethered grippers in unstructured environments," Journal of Micro-Bio Robotics, vol. 12, no. 1-4, pp. 45-52, 2017.

[214] J. Leclerc, A. Ramakrishnan, N. V. Tsekos, and A. T. Becker, "Magnetic hammer actuation for tissue penetration using a millirobot," IEEE Robotics and Automation Letters, vol. 3, no. 1, pp. 403-410, 2018.

[215] L. Dupourqué, F. Masaki, Y. L. Colson, T. Kato, and N. Hata, "Transbronchial biopsy catheter enhanced by a multisection continuum robot with follow-the-leader motion," International Journal of Computer Assisted Radiology and Surgery, vol. 14, no. 11, pp. 2021-2029, 2019. 
[216] A. Denasi and S. Misra, "Independent and leader-follower control for two magnetic micro-agents," IEEE Robotics and Automation Letters, vol. 3, no. 1, pp. 218-225, Jan 2018.

[217] J. Sikorski, C. M. Heunis, F. Franco, and S. Misra, "The ARMM system: An optimized mobile electromagnetic coil for non-linear actuation of flexible surgical instruments," IEEE Transactions on Magnetics, vol. 55, no. 9, pp. $1-9,2019$.

[218] M. Kaya, A. Denasi, S. Scheggi, E. Agbahca, C. Yoon, D. H. Gracias, and S. Misra, "A multi-rate state observer for visual tracking of magnetic microagents using $2 \mathrm{~d}$ slow medical imaging modalities," in Proceedings of the 2018 IEEE/RSJ International Conference on Intelligent Robots and Systems (IROS), 2018, pp. 1-8.

[219] R. M. Murray, Z. Li, and S. S. Sastry, A Mathematical Introduction to Robotic Manipulation, 1994, vol. 29.

[220] A. S. Deo and I. D. Walker, "Overview of damped least-squares methods for inverse kinematics of robot manipulators," Journal of Intelligent and Robotic Systems, vol. 14, no. 1, pp. 43-68, 1995.

[221] C. Nadeau, H. Ren, A. Krupa, and P. Dupont, "Intensity-based visual servoing for instrument and tissue tracking in 3d ultrasound volumes," IEEE Transactions on Automation Science and Engineering, vol. 12, no. 1, pp. 367-371, 2014.

[222] P. Chatelain, A. Krupa, and N. Navab, "3d ultrasound-guided robotic steering of a flexible needle via visual servoing," in Proceedings of the 2015 IEEE International Conference on Robotics and Automation (ICRA), 2015, pp. 2250-2255.

[223] A. Denasi, F. Khan, K. J. Boskma, M. Kaya, C. Hennersperger, R. Göbl, M. Tirindelli, N. Navab, and S. Misra, "An observer-based fusion method using multicore optical shape sensors and ultrasound images for magneticallyactuated catheters," in Proceedings of the 2018 IEEE International Conference on Robotics and Automation (ICRA), 2018, pp. 50-57.

[224] J. Edelmann, A. J. Petruska, and B. J. Nelson, "Estimation-based control of a magnetic endoscope without device localization," Journal of Medical Robotics Research, vol. 3, no. 01, p. 1850002, 2018.

[225] L. Yang, X. Du, E. Yu, D. Jin, and L. Zhang, "DeltaMag: An electromagnetic manipulation system with parallel mobile coils," in Proceedings of the 2019 International Conference on Robotics and Automation (ICRA), 2019, pp. 9814-9820.

[226] T. Xu, J. Zhang, M. Salehizadeh, O. Onaizah, and E. Diller, "Millimeterscale flexible robots with programmable three-dimensional magnetization and motions," Science Robotics, vol. 4, no. 29, 2019. 
[227] S. Scheggi, K. K. T. Chandrasekar, C. Yoon, B. Sawaryn, G. van de Steeg, D. H. Gracias, and S. Misra, "Magnetic motion control and planning of untethered soft grippers using ultrasound image feedback," in Proceedings of the 2017 IEEE International Conference on Robotics and Automation (ICRA), 2017, pp. 6156-6161.

[228] S. Jeon, S. Kim, S. Ha, S. Lee, E. Kim, S. Y. Kim, S. H. Park, J. H. Jeon, S. W. Kim, C. Moon, B. J. Nelson, J.-y. Kim, S.-W. Yu, and H. Choi, "Magnetically actuated microrobots as a platform for stem cell transplantation," Science Robotics, vol. 4, no. 30, 2019.

[229] J. Lapointe and S. Martel, "Thermoresponsive hydrogel with embedded magnetic nanoparticles for the implementation of shrinkable medical microrobots and for targeting and drug delivery applications," in Proceedings of the 2009 Annual International Conference of the IEEE Engineering in Medicine and Biology Society (EBMS), 2009, pp. 4246-4249.

[230] B. R. Donald, C. G. Levey, I. Paprotny, and D. Rus, "Planning and control for microassembly of structures composed of stress-engineered MEMS microrobots," The International Journal of Robotics Research, vol. 32, no. 2, pp. 218-246, 2013.

[231] V. Magdanz, M. Guix, and O. G. Schmidt, "Tubular micromotors: from microjets to spermbots," Robotics and Biomimetics, vol. 1, no. 1, pp. 1-10, 2014 .

[232] X. Yan, Q. Zhou, M. Vincent, Y. Deng, J. Yu, J. Xu, T. Xu, T. Tang, L. Bian, Y.-X. J. Wang et al., "Multifunctional biohybrid magnetite microrobots for imaging-guided therapy," Science Robotics, vol. 2, no. 12, 2017.

[233] P. Fischer, B. J. Nelson, and G.-Z. Yang, "New materials for next-generation robots," Science Robotics, vol. 3, no. 18, 2018.

[234] S. Pané, J. Puigmartí-Luis, C. Bergeles, X.-Z. Chen, E. Pellicer, J. Sort, V. Počepcová, A. Ferreira, and B. J. Nelson, "Imaging technologies for biomedical micro-and nanoswimmers," Advanced Materials Technologies, vol. 4, no. 4, p. 1800575, 2019.

[235] A. Servant, F. Qiu, M. Mazza, K. Kostarelos, and B. J. Nelson, "Controlled in vivo swimming of a swarm of bacteria-like microrobotic flagella," Advanced Materials, vol. 27, no. 19, pp. 2981-2988, 2015.

[236] N. Jaffrezic-Renault, C. Martelet, Y. Chevolot, and J.-P. Cloarec, "Biosensors and bio-bar code assays based on biofunctionalized magnetic microbeads," Sensors, vol. 7, no. 4, pp. 589-614, 2007.

[237] F. Ongaro, D. Niehoff, S. Mohanty, and S. Misra, "A contactless and biocompatible approach for $3 d$ active microrobotic targeted drug delivery," $M i$ cromachines, vol. 10, no. 8, p. 504, 2019. 
[238] M. T. Hou, H.-M. Shen, G.-L. Jiang, C.-N. Lu, I.-J. Hsu, and J. A. Yeh, "A rolling locomotion method for untethered magnetic microrobots," Applied Physics Letters, vol. 96, no. 2, p. 024102, 2010.

[239] A. Katouzian, E. D. Angelini, S. G. Carlier, J. S. Suri, N. Navab, and A. F. Laine, "A state-of-the-art review on segmentation algorithms in intravascular ultrasound (IVUS) images," IEEE Transactions on Information Technology in Biomedicine, vol. 16, no. 5, pp. 823-834, 2012.

[240] T. Yamakawa, T. Inoue, Y. He, M. Fujii, M. Suzuki, and M. Niwayama, "Development of an implantable flexible probe for simultaneous near-infrared spectroscopy and electrocorticography," IEEE Transactions on Biomedical Engineering, vol. 61, no. 2, pp. 388-395, 2014.

[241] S. Hetts, M. Saeed, A. Martin, L. Evans, A. Bernhardt, V. Malba, F. Settecase, L. Do, E. Yee, and A. Losey, "Endovascular catheter for magnetic navigation under MR imaging guidance: Evaluation of safety in vivo at 1.5 T," AJNR Am. J. Neuroradiol., vol. 34, no. 11, pp. 2083-2091, 2013.

[242] T. Liu, R. Jackson, D. Franson, N. L. Poirot, R. K. Criss, N. Seiberlich, M. A. Griswold, and M. C. Çavuşoğlu, "Iterative jacobian-based inverse kinematics and open-loop control of an MRI-guided magnetically actuated steerable catheter system," IEEE/ASME Transactions on Mechatronics, vol. 22, no. 4, pp. 1765-1776, 2017.

[243] F. M. Sánchez-Margallo, F. J. Pérez-Duarte, J. A. Sánchez-Margallo, M. Lucas-Hernández, A. M. Matos-Azevedo, and I. Díaz-Güemes, "Application of a motion capture data glove for hand and wrist ergonomic analysis during laparoscopy," Minimally Invasive Therapy $\&$ Allied Technologies, vol. 23, no. 6, pp. 350-356, 2014.

[244] C. M. Heunis, B. B. F. M. Barata, G. P. Furtado, and S. Misra, "Collaborative surgical robots: Optical tracking during endovascular surgery," IEEE Robotics $\mathscr{G}$ Automation Magazine, 2020.

[245] F. Khan, A. Denasi, D. Barrera, J. Madrigal, S. Sales, and S. Misra, "Multicore optical fibers with bragg gratings as shape sensor for flexible medical instruments," IEEE Sensors, vol. 19, no. 14, pp. 5878-5884, 2019.

[246] O. Cogal and Y. Leblebici, "An insect eye inspired miniaturized multi-camera system for endoscopic imaging," IEEE transactions on Biomedical Circuits and Systems, vol. 11, no. 1, pp. 212-224, 2016.

[247] C. Della Santina, R. K. Katzschmann, A. Bicchi, and D. Rus, "Model-based dynamic feedback control of a planar soft robot: Trajectory tracking and interaction with the environment," The International Journal of Robotics Research, vol. 39, no. 4, pp. 490-513, 2020.

[248] N. Lepora and J. Lloyd, "Optimal deep learning for robot touch: Training accurate pose models of 3d surfaces and edges," IEEE Robotics 65 Automation Magazine, 2020. 
[249] F. Khan, R. J. Roesthuis, and S. Misra, "Force sensing in continuum manipulators using fiber bragg grating sensors," in Proceedings of the 2017 IEEE/RSJ International Conference of Robotics and Systems (IROS), 2017, pp. 2531-2536.

[250] K. M. Park, Y. M. Shin, K. Kim, and H. Shin, "Tissue engineering and regenerative medicine 2017: a year in review," Tissue Engineering Part B: Reviews, vol. 24, no. 5, pp. 327-344, 2018.

[251] Y.-Q. Xie, L. Wei, and L. Tang, "Immunoengineering with biomaterials for enhanced cancer immunotherapy," Wiley Interdisciplinary Reviews: Nanomedicine and Nanobiotechnology, vol. 10, no. 4, p. e1506, 2018.

[252] I. C. Yasa, H. Ceylan, U. Bozuyuk, A.-M. Wild, and M. Sitti, "Elucidating the interaction dynamics between microswimmer body and immune system for medical microrobots," Science Robotics, vol. 5, no. 43, 2020.

[253] A. E. Chen and D. A. Melton, "Derivation of human embryonic stem cells by immunosurgery," Journal of Visualized Experiments, no. 10, p. e574, 2007.

[254] I. C. Yasa, A. F. Tabak, O. Yasa, H. Ceylan, and M. Sitti, "3d-printed microrobotic transporters with recapitulated stem cell niche for programmable and active cell delivery," Advanced Functional Materials, vol. 29, no. 17, p. 1808992, 2019. 



\section{Acknowledgements}

I am sure this is the part of the dissertation that many of you will read with the greatest care, hence the pressure I feel while typing this section is also the greatest. Working in a relatively exotic subject, I sometimes cannot help but to ask myself whether what I do is relevant at all. It certainly would not be, if not for the people like you, who bother to be my audience, even for a short while. Thus, I would first want to thank you - my reader - whoever you are, and whatever the reason that brought you here, for being interested in what I have to say. Without people like you, I might have as well just slept through the last five years.

Of course, none of my work would be possible if not for the extraordinary amount of trust and support given to me by my supervisor, Prof. Dr. Sarthak Misra. I still remember the time I first spoke with you, and, how afterwards, I was sure I would not get this position. To my surprise, you did accept me as your student and, since then, you have always vouched for me, while I was on my way to pursue all the crazy ideas I discuss in this dissertation. Thanks a lot for giving me a chance to test myself and to grow up in the intense world-class intellectual environment you have created. It was the fun I needed. I would get bored otherwise.

Speaking of the environment; my stay at SRL would be in vain, if not for all the extraordinary people from all over the world who have shared this experience with me. Big up to all my former and current colleagues for the intellectual and social fun they have provided, in order of appearance. Momen: we have spoken only briefly, but thanks for letting me join your defence and dinner, thus allowing me to start my PhD journey by seeing what awaits at the end. Fede: I have never met another person like you, and I think it's a good thing - the world needs only one superhero. Thanks for all the time we spent together rambling around Enschede and beyond. It was a blast and I could not have asked for a better office mate. Stefano: the sheer extent of what I have learnt from you about academia will keep me feeling grateful for years to come. I am continuously rediscovering the meaning of things you were telling me when we worked together. Alper: your knowledge is as impressive as your patience and as intimidating as your reviews. Thanks for letting me into the control world and for teaching me how to speak maths, even though I'm sure I still suck at both. Pedro: you were always my beacon of sanity, whenever my $\mathrm{PhD}$ was getting into my head too much. And you were right, I did get better at Adobe suite. Klaas Jelmer: your work has spared me an awful lot of struggle. Thanks for having me at UMCG and for your

selfless help with Sonix Touch when I needed it the most. Guus: your stoicism and sense of humour is exceptional, and the robotic gear you have built was always 
making me jealous. Above all, thanks for teaching me the true meaning of the word "tomorrow". Roy: you were probably the first Twentse person with whom I got to talk for a longer amount of time, and your setup was the first functional robot I have worked with. Sorry I was not much help back then. Navid: your shining smile should be patented, so that after a decade everyone could wear it. Thanks for all the control trips to Utrecht, they were one of the highlights of my second year. Gert: thanks for always being the adult in the room, be it physically or via WhatsApp, and for the invaluable tips regarding Dutch traffic regulations. Fouzia: if I needed a real diplomat, I would ask for your help. Thanks for constantly making me realise the extent of my impolitic demeanour, and for invaluable help when I joined the EDEN family. Christoff: oh boi, I don't even know what to say. You're the best partner in crime. Without you, I would have probably already ended up insane, mauled to death by some Reviewer \#2, or crushed by a pile of M5 and M6 screws, while trying to find this one Allen key we all need. Thank you for patiently saving me from all of that, and for having me in the best braai circle north of the equator. Mert: thanks for trying to teach me the Turkish sense of humour, which I still don't understand. And for a virtually infinite stream of goodies, be it tea, candy, computer vision code or laboratory equipment, which has saved me on numerous occasions. Ugo: your perspective on science and on the microscale world has really helped me define where I want to go with my research. A pity that you chose the Flemish over us, but your spirit will always live on in SRL, playing the circle game with naive, knowledge-thirsty students. Sumit: now when I started talking about microscale, it was obvious that you'd pop up. And the answer is always YOS. Thanks for everything, starting from all the microrobotic research you have made me aware of, followed by our shared appreciation of all things floofy, ending with that @Daily__Goats profile you shared with me today. I keep my fingers crossed for boofers and citations alike coming your way en masse. Venkat: the know-how you have brought into the lab and developed further is something remarkable. Few people can make plastic exercise and dance repeatably. But what I find even more impressive is how repeatably you can make your students achieve great things. Thanks for being a source of inspiration, for the amazing hardware you always manage to find, and for delicious Indian food. Guilherme: even though you left suddenly, I would be surprised if this wasn't all just temporary. Once I get wherever you are now, I hope we can talk again about politics and control theory. I'm sure you will have all the answers figured out by then, and I can learn from you again, like I used to. Theodosia: thanks for your kindness, smile and readiness to recover information from my unstructured quasi-technical monologues. And for the general soothing effect you have on the people inside SRL. Zhengya: your arrival has added a completely new dimension to SRL, breathing new life into our UMCG satellite. Thanks for your enthusiasm about my work, and for your unbeatable persistence and resourcefulness. Islam: your accomplishments speak for themselves and your ability to patiently and kindly guide countless students through the labyrinths of robotics is something that will always make me impressed and a bit unsure about myself and my bad temper. Thank you for being an example, and for proving me wrong about the amount of things we can fit in $\mathrm{W}-130$. The lab looks great now. Morteza: your politeness can only match your ability to successfully handle 
matters that could otherwise be deemed a lost cause. Thanks for being with us, and for the fact that once you had arrived, the hissing sounds became much more pleasant. Jesus: I wish one could study and replicate the aura of kindness that surrounds you. Thanks for taking good care of BigMag and for the dank memes and wholesome content you distribute online. And please ensure the pom get enough pets and snacks. Yiyang: you were the first person in SRL I have seen working with machine learning. Thanks for bringing us closer to that world. And thank you for demonstrating that one can naturally oscillate with their outfit from academic to fashion icon and back. We need that attitude more widespread at the UT. Chuang: your infectious positive energy and enthusiasm are one of a kind. Thanks for that, I always feel energized after we talk. Filip: thanks for all the professional and private discussions we have had in the last few months. I have already managed to learn a lot from you, and I am drooling while I look at the hardware you have brought into SRL. And your openness, friendliness and a sense of humour remind me of all the best things Eastern Europe has to offer. Edwin: last but not least, thanks for your moderating influence on me, while I was emotionally struggling to get my last set of results for this thesis. And for calling me a wizard, I like that way of looking at my career choice.

A special place in my heart will always be occupied with respect and gratefulness to all the students, who have worked under my supervision. Imro: thanks for your patience, when I was still working things out, and your professional and engaged approach, which has taught me a lot. I wish we met a bit later, but I'm still very happy that I got to know you. Federico: your engineering skills were exceptional, and so was your independence. You could have as well been my colleague. Or my electronics lead. Giuseppe: your help with my first journal paper was invaluable. Thanks for handling with grace every problem I threw at you. And I'm sure no one will forget the precious attitude you were famous for. Maybe that is why legends about you were still circulating around Enschede long after you left. Rafic: the people person, the heart of the team. Your kindness, enthusiasm and the natural ability to click with others have always impressed me. Thanks for successfully tackling the broadest of my projects. And for figuring out that silicon tubes are transparent to ultrasound, which saved a lot of SRL members from distress. Etienne: "oh man", what a journey it was. You gave me a breakthrough in my research, a straight conference paper and no opportunity to proof-read your thesis before submission. Either way, it's you who's the man now. I am eternally grateful you decided to embark with me on this one. Oskar: seeing your drive and your intellect I could not help but to miserably look back at myself that age. A pity you didn't stay in robotics, but I hope you're happy where you are now. Victorien: the research conclusions of your internship were as unexpected as your appearance. You spared me months of going in circles with torpedoes that do not move. Thanks for that and for all the banter.

Neither the professional part of my acknowledgements, nor my professional life in general would be complete without the two guardian angels always present to dig me out from under a pile of paperwork, brighten my day with a smile, and offer me a few spare pens and whiteboard markers from the cabinet of abundance. Thank you Jeanine and Lianne for running the best secretariaat at the entire UT and for always being there to assist me with my issues. Without you two, the 
entire department would have collapsed ages ago (or at least I would).

Z całego serca chcę też podziękować mojej rodzinie, a w szczególności moim rodzicom: Teresie i Wiesławowi. Bez Waszego wsparcia i miłości, a także bez wartości, których (z różnym skutkiem) uczyliście mnie przez całe życie, nie stałybym dzisiaj w tym miejscu z podniesioną głową. Wielkie dzięki za opiekę, wyrozumiałość, dobre słowo i za nieustanną wiarę we mnie. Kocham Was nad życie i cieszę się, że razem możemy świętować ten sukces.

Osobne podziękowania należą się mojej siostrze Marii, na którą zawsze mogę liczyć. Dzięki za dawanie dobrej beki zawsze, przednie foty puchatych flooferów, szacunek do największego polaka, najlepsze techenko (\#babiczstyle) i tak wyborną gościnę na Śląsku, że nawet w tej chwili nie mogę się doczekać, aż znowu wpadnę Cię podenerwować swoim nieogarnięciem. Bez Twojego wsparcia i dobrej rady byłoby mi znacznie ciężej w życiu. Jestem zawsze maks dumny z Ciebie i trzymam kciuki za Twoje sukcesy tak mocno, że prawie mi odpadają z niedokrwienia. Czapki z głów, Pani Doktor!

When I think of the family, I could not omit my sista from another mista. Lusia, big thank you for all these years of KDL worldwide and for your unbeatable expertise in life, wiksa and beyond. I write this in English because I can, because you're a native South Londoner by now anyways (as certified by the medal you got), and to show off that thanks to you I also sometimes have a chance to be that metropolitan myself. I would not be able to tell the number of precious events, that have happened thanks to your doing, and of the extraordinary people I have met through you. I am super lucky to be related to you, even though I am not really. Pieseł stobo!

Piątka dla wszystkich moich polskojęzycznych znajomych, z którymi udało mi się utrzymać kontakt przez te zwariowane lata. Dla ludzi z czasów liceum: Adamczyka, Iwa, Magdy, Cytryny, Gostyka, Pameli, Martyny, Karoli - dzięki, że jest mi nadal dane widzieć z doskoku, jak rozwijają się Wasze historie. Dla znajomych ze studiów w Glasgow: Patoczki, Ozimka, Roksi i Kyla, Kacpra - mam nadzieję, że do zobaczenia gdzieś niebawem! Dla całej masy świetnych ludzi z innych miejsc: Kasi i Marczałke, Osy, Kipki, Utka, Tomka, Ziucia, Krynia, Dominika. Jestem pewien, że o kimś zapomniałem, ale mam nadzieję, że mi wybaczycie.

Thank you to all the fabulous people from the entire world, whom I had a pleasure to interact with in the last 5 years. Guido, Francesco, Luigi, Federica, Dimitri and the rest of the famous "Italian gang" of Enschede. Vasilis for our ongoing research chat that really makes me want to look back at biology. Jiena and Tim for the rooftop sessions and the homey British vibe you always bring around. Henrike for providing ongoing support to my best boi. Fabio, Gionata, Irfan and Asad for the best company I could possibly have had in Singapore. Diego for all the flamingos, an unforgettable New Year's Eve and one of the best birthday gifts I have ever received. Kostas, Stergios, Wolfgang, Frank and Arvid for $100 \%$ exquisite banter that one special week our department has taken over the stadium and the city, and for all the fun at ET-BE borrels. Stefano G. and Riccardo for your hospitality at Imperial and for reminding me of the charm of how things are done across the ditch. Tommi for being a friend to probably the best woman on this planet (as discussed later in this section) and for a blast 
in Berlin and Brussels. Tom for showing me around KL and saving me from a cabin fever, which I got after not talking to anyone for a week. Michieltje, hartelijk bedankt voor een onverwachte vriendschap, lekkere eten en een zeldzame mogelijkheid om mijn Nederlands comfortabel te oefenen.

Special thanks for Ms. Alina Płoszaj, who managed to make me stop and take a breath, when I was running blindly in a wrong direction. You taught me how to look around to see better where I stand now and where I want to go next. Without your help, my cart would lose its wheels a while back, and I would definitely not be finishing this thesis today. I am extremely obliged.

Finally, I would want to thank, give a written hug to, and make a silly dance for the wonderful lady that has been giving flavour to my life for the last $7+$ years. Janine, I am not sure where I would have ended up without you, but I know that my English, my cooking skills, and my sense of aesthetics would all have suffered irreparable damage, if not for your endearing everyday efforts to civilise the rugged beast I am. From Dublin to Narita, from Salla to Tarifa, all the memories, you keep on sharing with me, make me a happy man constantly looking forward to our next adventure, even if that means just a cozy bike trip around Enschede. Thanks for all the fishe, for sorting my life and bearing with me through good and bad, and for giving me a reason to wake up (even though naps next to you typing violently are equally amazing). I know your Finnish soul is probably already overwhelmed with the amount of sweet garbage I just disclosed publicly. I end then. I love you. 



\section{About the Author}

Jakub Sikorski was born on the 25th of October 1992 in Duszniki-Zdrój, Poland in a remote, picturesque spa town in the foothills of the Sudetes. He received an International Baccalaureate Diploma from the 5th High School, Wrocław, Poland, and an M.Eng degree in Biomedical Engineering from the University of Glasgow, Glasgow, United Kingdom. During his studies in Glasgow he completed two summer research internships at the Institute of Biomedical Engineering, Technion - Israel Institute of Technology, Haifa, Israel, and at the Institute of Mathematics, Academy of Sciences of the Republic of Tajikistan, Dushanbe, Tajikistan. Between June and December 2014, he visited the Laboratory for Orthopaedic Technology at ETH Zürich, Zürich,

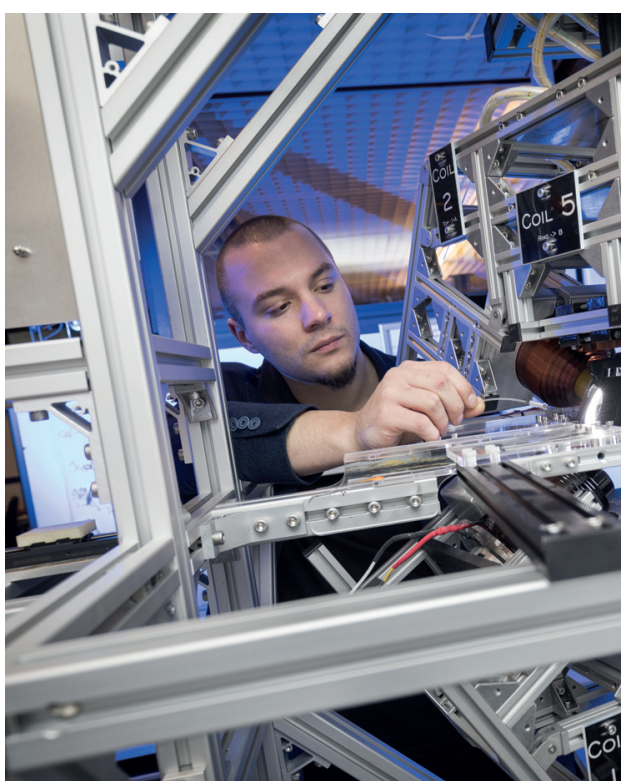

Jakub and BigMag. December 2017 Switzerland, to conduct his master's research project in computational biomechanics under the supervision of Prof. Dr. Stephen J. Ferguson and Prof. Dr. K. Elizabeth Tanner.

Driven by interest in both automation and medical sciences, in September 2015 he joined the Surgical Robotics Laboratory (SRL, surgicalroboticslab.nl) at the University of Twente, the Netherlands to pursue a doctoral degree under the supervision of Prof. Dr. Sarthak Misra. Here, he has been working on design, modelling and control of magnetically-actuated catheters. Additionally, since September 2019, he is affiliated with University Medical Centre Groningen, Groningen, the Netherlands, where he works on shape reconstruction technology for programmable flexible needles.

During his time at SRL, Jakub has developed strong research interest in mechatronic design of medical robotic systems. He has learned how to make inanimate objects move and how to speak fluent $\mathrm{C}++$. The thesis you are reading is the direct outcome of 5 years of his research. 